ESCUELA TÉCNICA SUPERIOR DE INGENIERÍA AGRONÓMICA, ALIMENTARIA Y DE BIOSISTEMAS

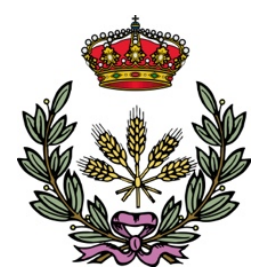

\title{
Synthetic Biology efforts in engineering nitrogenase Fe protein biosynthesis in plastids
}

TESIS DOCTORAL

\author{
ÁLVARO ESEVERRI SABATÉ \\ GRADUADO EN BIOTECNOLOGÍA
}





\section{POLITÉCNICA}

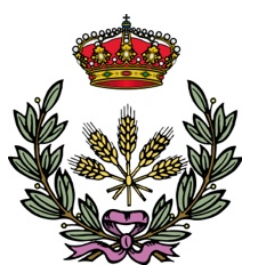

PROGRAMA DE DOCTORADO EN BIOTECNOLOGÍA Y RECURSOS GENÉTICOS EN PLANTAS Y MICROORGANISMOS ASOCIADOS

DEPARTAMENTO DE BIOTECNOLOGÍA-BIOLOGÍA VEGETAL

Synthetic Biology efforts in engineering nitrogenase Fe protein biosynthesis in plastids

\section{TESIS DOCTORAL}

AUTOR: ÁLVARO ESEVERRI SABATÉ

GRADUADO EN BIOTECNOLOGÍA

DIRECTORA: ELENA CARO BERNAT

DOCTORA EN BIOLOGIA MOLECULAR Y BIOQUIMICA

CO-DIRECTOR: LUIS M. RUBIO HERRERO

DOCTOR EN BIOLOGÍA 



\section{POLITÉCNICA}

\section{UNIVERSIDAD POLITÉCNICA DE MADRID}

Tribunal nombrado por el Magfco. Y Excmo. Sr. Rector de la Universidad Politécnica de Madrid, el día de de 2020.

Presidente: Dr. Fernando Ponz Ascaso

Secretario: Dr. Luis Rey Navarro

Vocal: Dra. Janneke Balk

Vocal: Dra. Nicola Patron

Vocal: Dr. Francisco Barro Losada

Suplente: Dr. David Durán Wendt

Suplente: Dra. Virginia Ruiz Ferrer

Realizado el acto de defensa y lectura de Tesis el día de de 2021 en el Centro de Biotecnología y Genómica de Plantas (CBGP, UPM-INIA).

EL PRESIDENTE LOS VOCALES 

"What I cannot create, I do not understand"

- Richard Feynman 

A mis padres 



\section{Table of Contents}

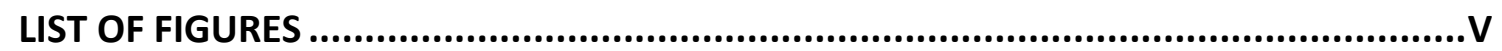

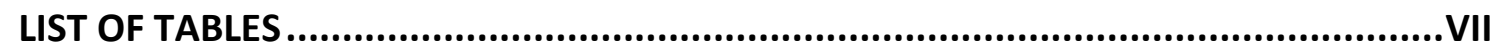

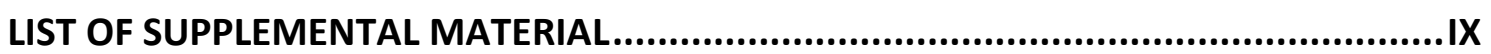

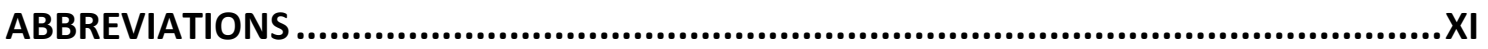

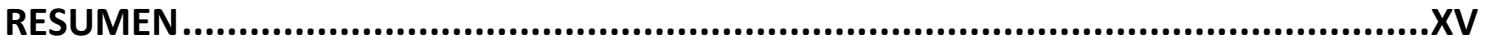

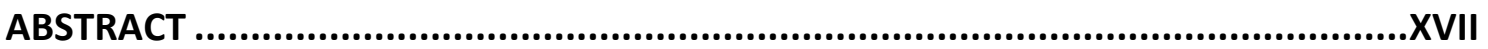

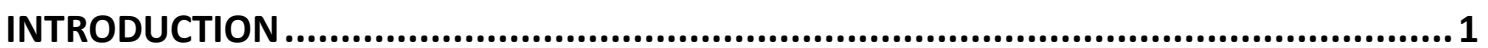

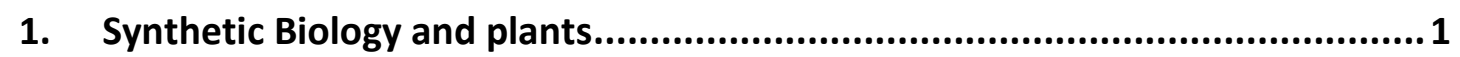

1.1. Introduction to Synthetic Biology.......................................................... 1

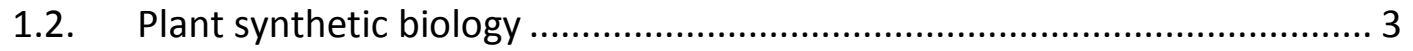

1.3. The DBTL process in plant SynBio........................................................... 4

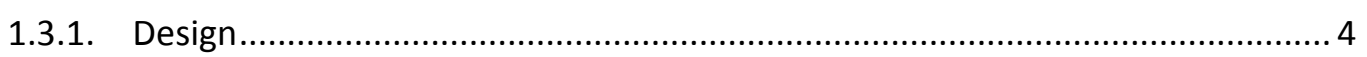

1.3.1.1. Outside parameters to optimize during design ....................................... 4

1.3.1.2. Inside parameters to optimize during design ........................................... 6

1.3.1.3. Computational tools for design ............................................................ 9

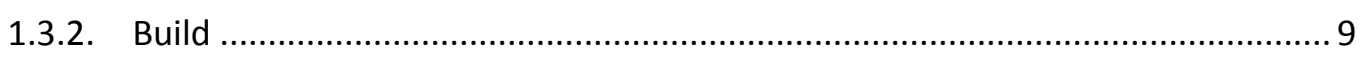

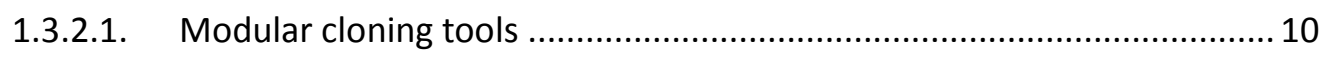

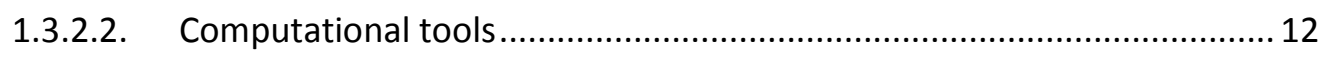

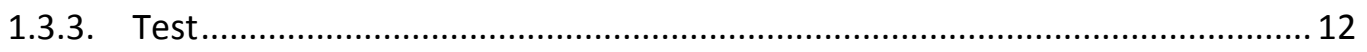

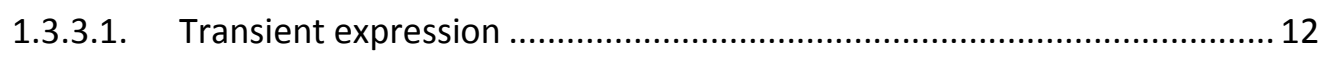

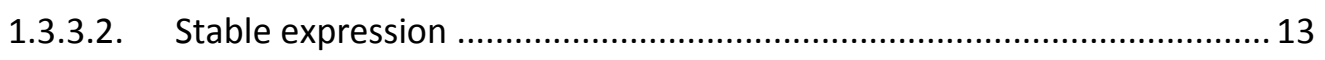

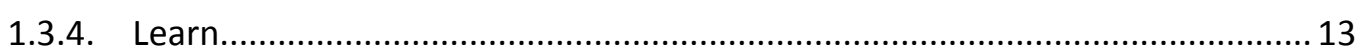

2. Application of SynBio tools to the engineering of Biological Nitrogen Fixation

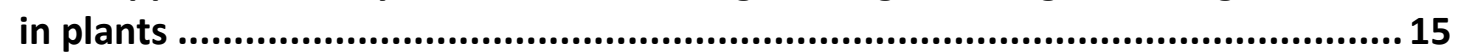

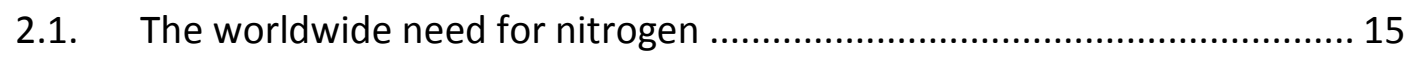

2.2. Biological nitrogen fixation as an alternative ......................................... 15

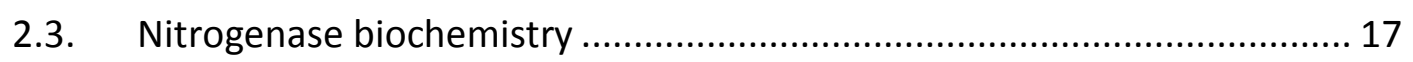

2.4. Engineering eukaryotic nitrogen fixation ................................................ 20

2.5. Coupling nitrogen fixation to plant metabolism ................................... 21

2.6. State of the art in the engineering of nitrogen-fixing organisms.............. 22

2.6.1. Engineering nitrogen fixation in a non-diazotrophic cyanobacterium ............. 22

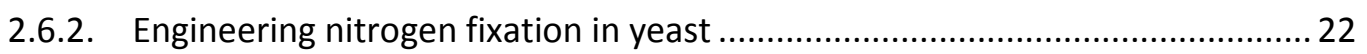

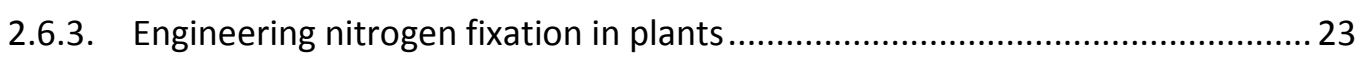

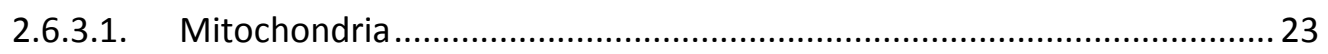

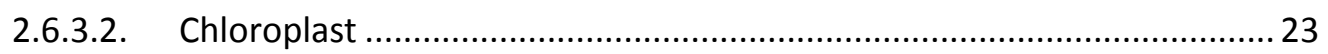

2.7. Engineering of nitrogenase biosynthesis in plastids .............................. 24

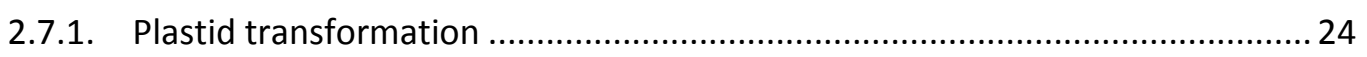

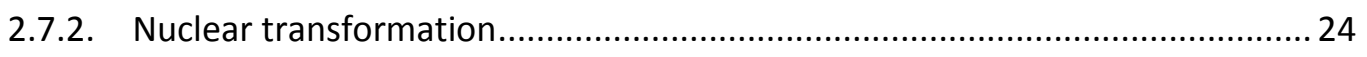




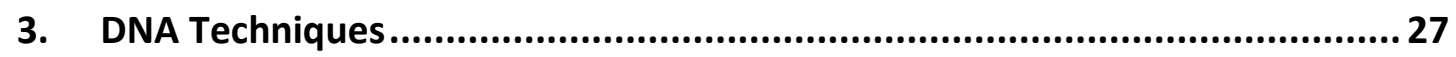

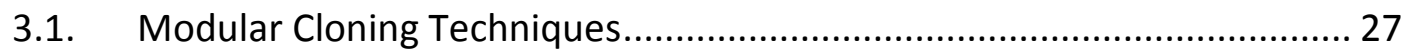

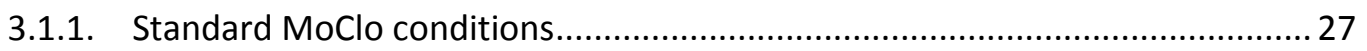

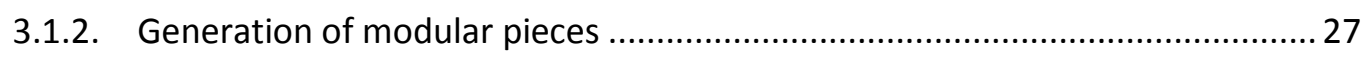

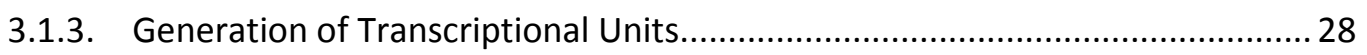

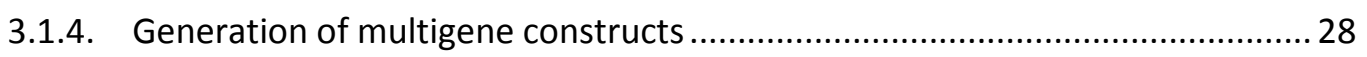

3.1.5. Adaptation of MoClo vectors for particle bombardment ................................ 29

3.2. Multiple Overlap Extension PCR ........................................................... 29

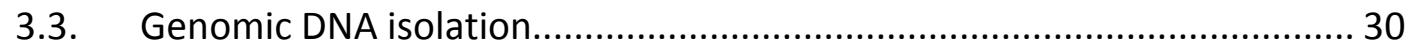

4. Bacterial Culture Techniques ....................................................................... 30

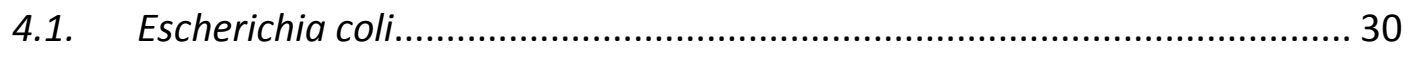

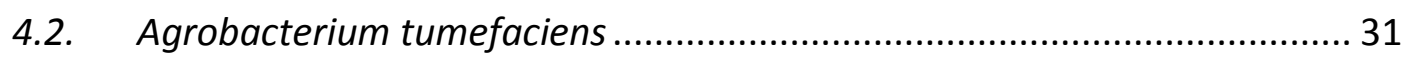

5. Plant Culture Techniques..................................................................... 31

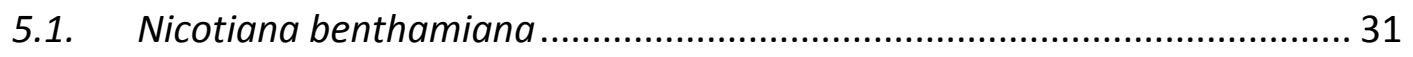

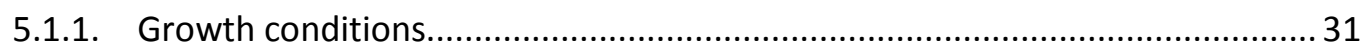

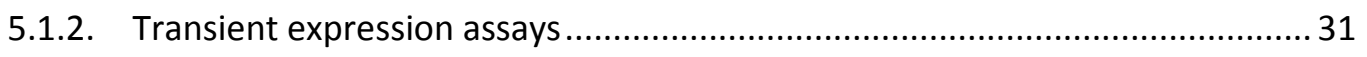

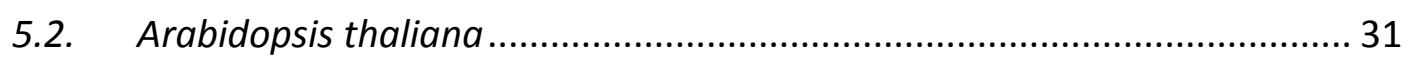

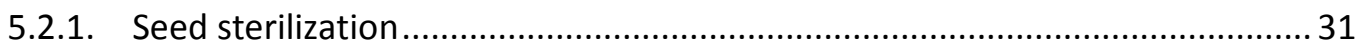

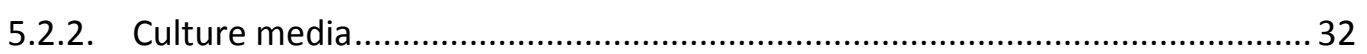

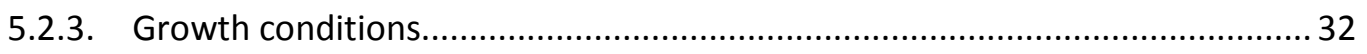

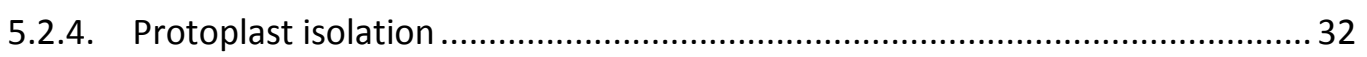

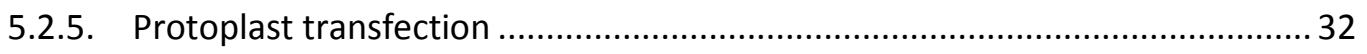

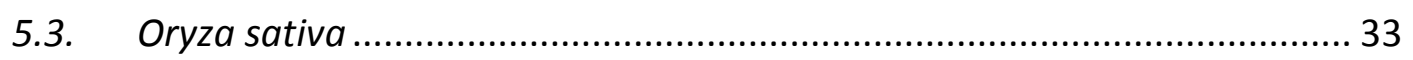

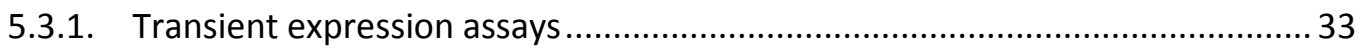

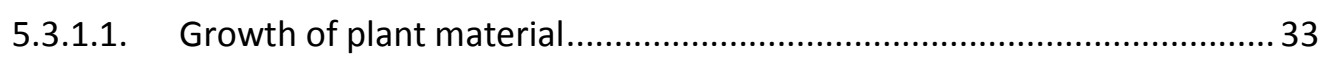

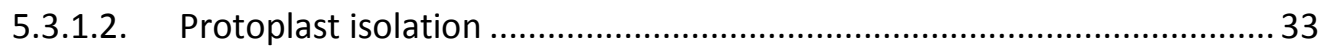

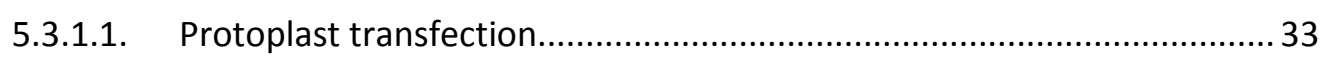

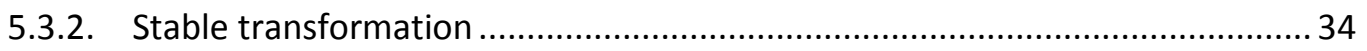

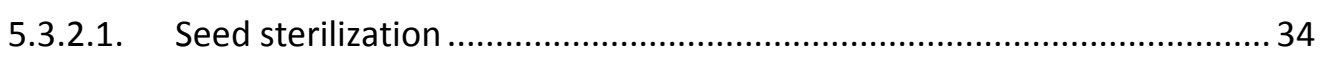

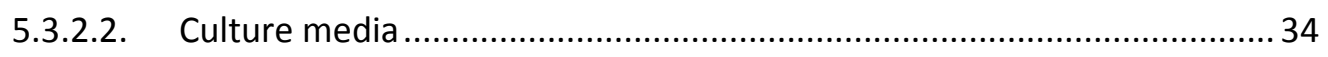

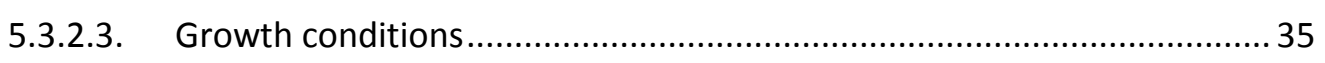

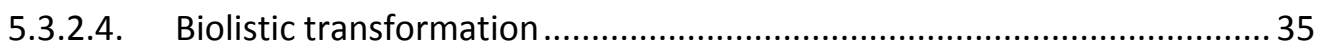

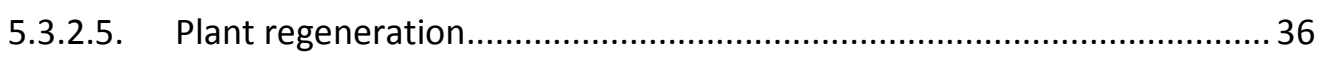

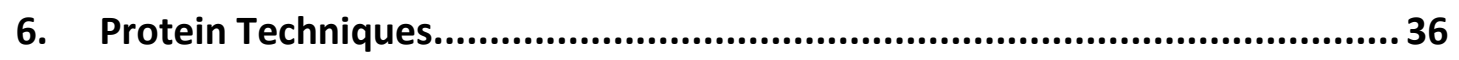

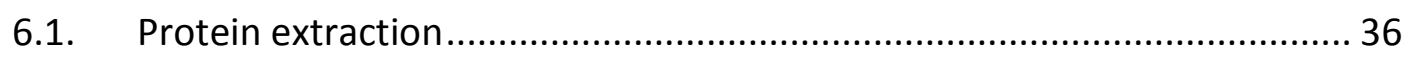

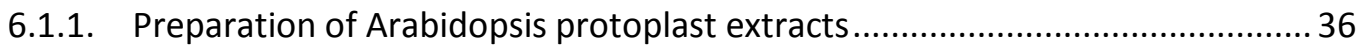

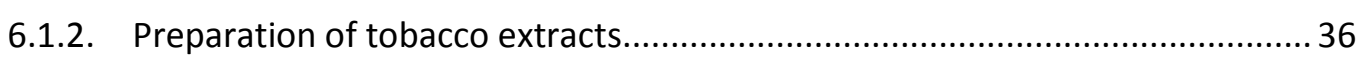

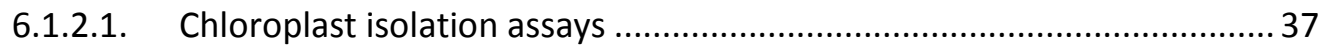

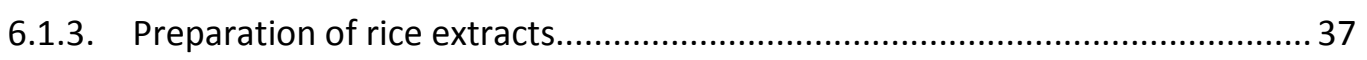




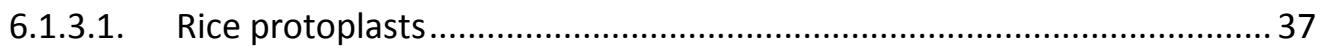

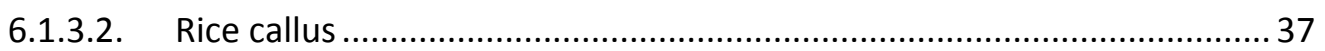

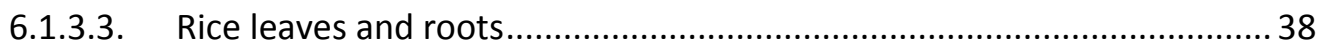

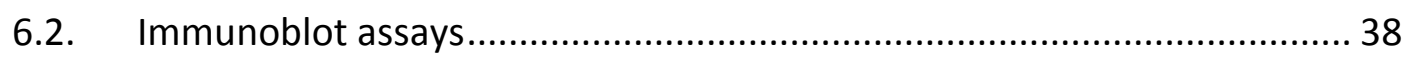

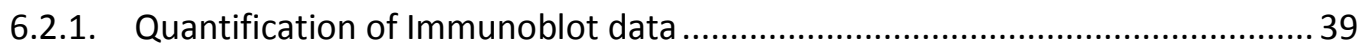

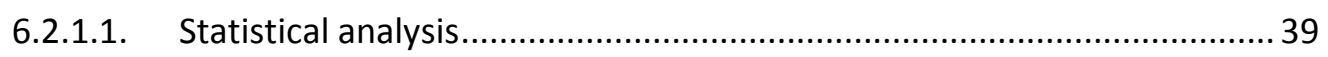

6.3. Anaerobic purification of TwinStrep-tagged $\mathrm{NbNifH}$............................... 39

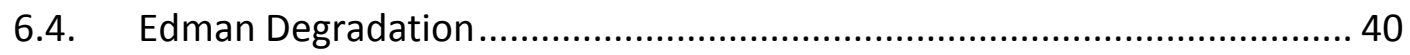

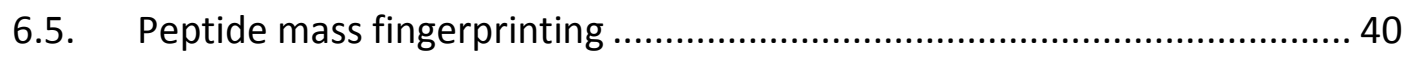

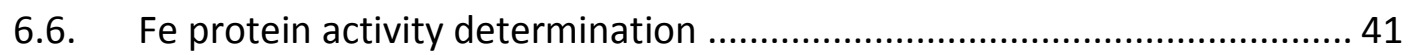

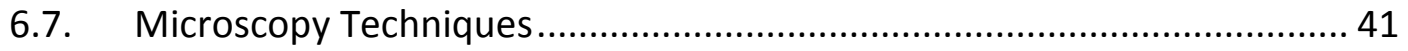

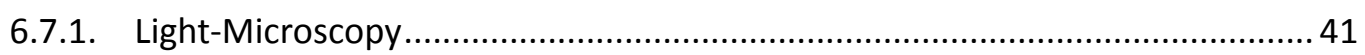

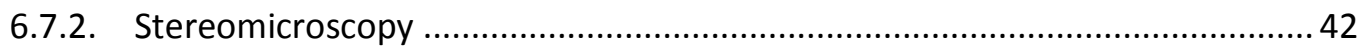

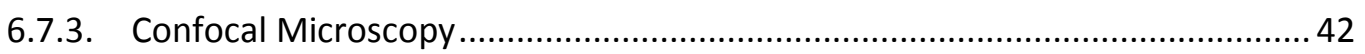

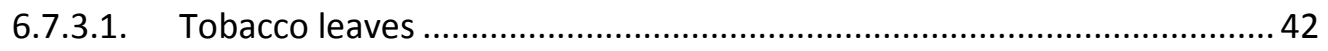

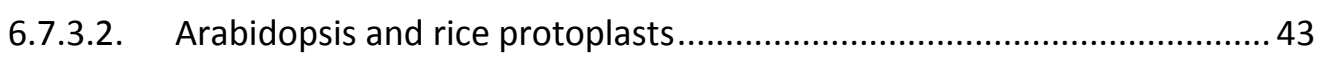

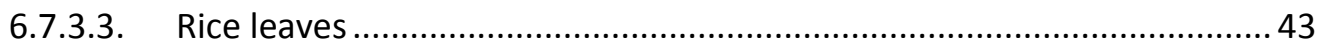

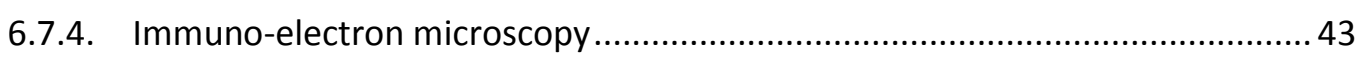

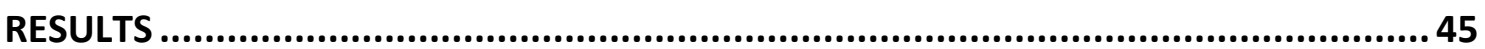

7. Use of synthetic biology tools to optimize the production of active nitrogenase Fe protein in chloroplasts of tobacco leaf cells ............................... 45

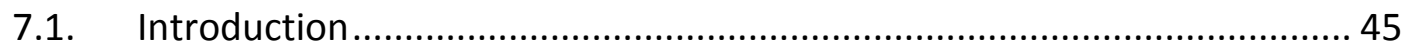

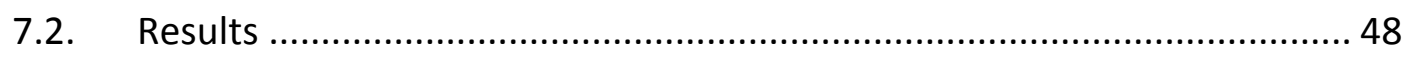

7.2.1. nif gene synthetic design ......................................................................... 48

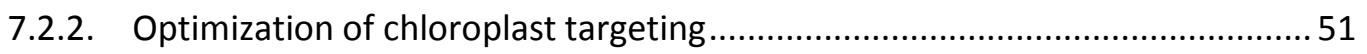

7.2.3. Tobacco plants co-expressing nifH, nifM, nifU, and nifS exhibit Fe protein activity 57

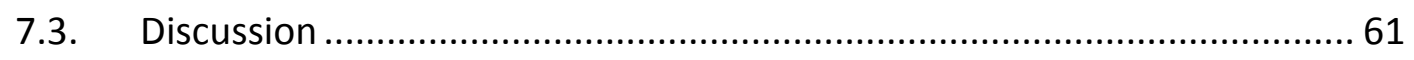

7.3.1. Optimization of nif gene synthetic design and chloroplast targeting................61 61

7.3.2. Purification of active $\mathrm{NifH}$ from tobacco chloroplasts .................................... 63

8. Optimization of nitrogenase Fe protein biosynthesis in rice: Arabidopsis transit peptides as tools for trait engineering in rice plastids ............................65

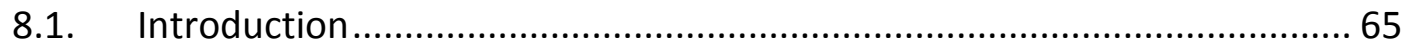

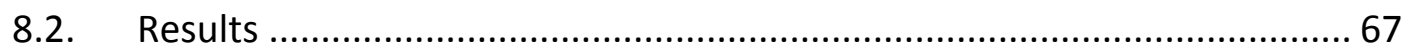

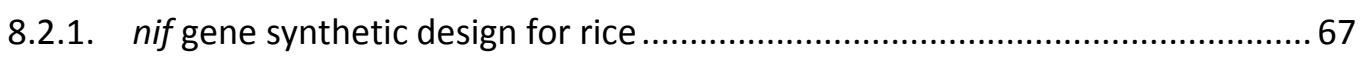

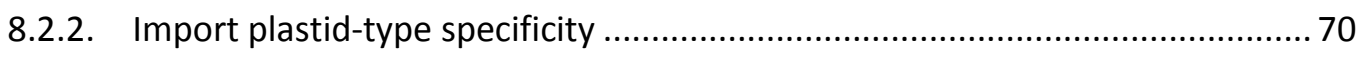

8.2.3. Conservation of the import mechanism between species ................................ 76

8.2.4. Arabidopsis TPs as tools for plastid import in whole rice plants ....................... 78

8.2.5. NifM remains a requirement for $\mathrm{NifH}$ solubility in rice .................................. 83

8.2.6. Stable transformation of rice embryos co-expressing nifH, nifM, nifU, and nifS 84 


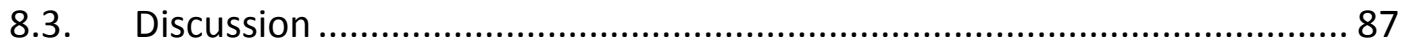

8.3.1. Optimization of $\mathrm{NifH}$ biosynthetic pathway in rice plastids............................. 87

8.3.2. Arabidopsis TPs as tools for plastid import..................................................... 88

8.3.3. Efforts in the generation of nif expressing rice lines ..................................... 90

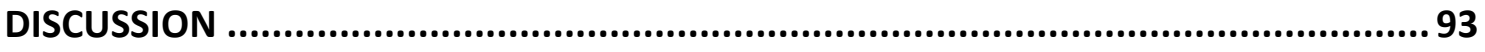

Azotobacter $n$ if gene optimization for expression in higher plants......................93

Optimization of protein import into plastids ..................................................... 94

Engineering nitrogenase Fe protein biosynthesis in plastids ..............................96

Perspectives of nitrogen-fixing plants ...........................................................97

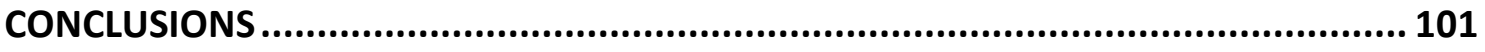

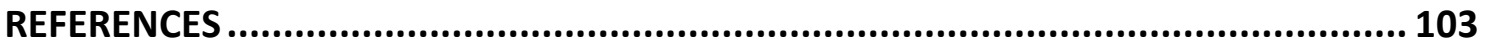




\section{List of Figures}

Figure 1. The design-build-test-learn (DBTL) cycle in synthetic biology................................... 2

Figure 2. Schematic overview of outside features affecting synthetic gene expression.............. 5

Figure 3. Schematic overview of inside features affecting synthetic gene expression. …........... 7

Figure 4. Workflow followed in Codon Optimization OnLine. ................................................ 9

Figure 5. Overview of Golden Gate MoClo Assembly Standard............................................... 11

Figure 6. Biological nitrogen fixation in cereal crops as an alternative to industrial fertilizers. 17

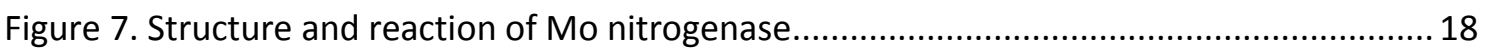

Figure 8. Simplified model of Fe protein maturation and its roles in MoFe protein maturation. 19

Figure 9. Effect of using synthetically designed nif genes optimized for tobacco...................... 49

Figure 10. Time course accumulation of synthetically designed nif genes optimized for tobacco.

Figure 11. Screening of chloroplast transit peptides fused to NifH, NifM, NifU, and NifS proteins. 52

Figure 12. Confocal microscopy localization of stroma-targeted Nif proteins. ...........................53

Figure 13. Subcellular fractionation confirming the localization of stroma-targeted Nif proteins. 55

Figure 14. Expression of nifH alone leads to protein accumulation in foci originating from

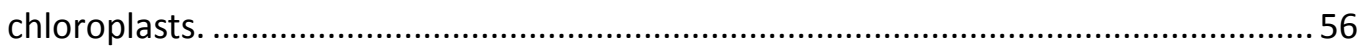

Figure 15. NifM is necessary for the accumulation of soluble $\mathrm{NifH}$ in the stroma. .....................5 57

Figure 16. Co-expression of nifH, nifM, nifU, and nifS in chloroplasts of $N$. benthamiana. ....... 58

Figure 17. Purification of $\mathrm{NifH}$ from chloroplasts of $N$. benthamiana co-expressing nifH, nifM, nifU, and nifS. 59

Figure 18. Purification of NifH from chloroplasts of $N$. benthamiana co-expressing nifH and nifM.

Figure 19. Effect of using diverse nifH and nifM gene optimizations in rice. ............................69

Figure 20. nifU and nifS gene optimizations led to no detectable protein in rice. ...................... 70

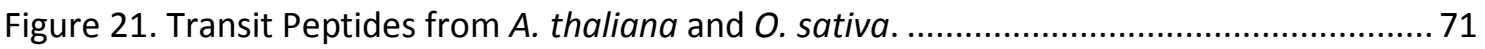

Figure 22. In vivo chloroplast targeting of TP-eGFP fusion proteins in Arabidopsis leaf

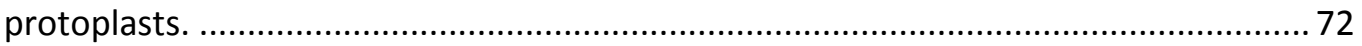

Figure 23. In vivo leucoplast targeting of TP-eGFP fusion proteins in Arabidopsis root protoplasts.

Figure 24. Import efficiency of $A$. thaliana and $O$. sativa Transit Peptides in Arabidopsis leaf protoplasts. 74

Figure 25. Import efficiency of $A$. thaliana and $O$. sativa Transit Peptides in Arabidopsis root protoplasts.

Figure 26. Arabidopsis TPs are able to direct eGFP into rice chloroplast. .................................. 77

Figure 27. Import efficiency of $A$. thaliana and 0 . sativa Transit Peptides in rice protoplast.... 78

Figure 28. Stably transformed rice lines accumulate eGFP in callus stage. ...............................79

Figure 29. Stably transformed rice callus lines exhibit eGFP targeting into rice pro-plastids by Arabidopsis TPs. 80

Figure 30. Regenerated rice plants show eGFP targeting into chloroplast driven by Arabidopsis TPs. 
Figure 31. Roots from regenerated rice plants show eGFP targeting into leucoplasts driven by Arabidopsis TPs.

Figure 32. NifM remains necessary for the accumulation of soluble NifH in the stroma of rice cells.

Figure 33. Multigenic constructs carrying nifH, nifM, nifU, and nifS for rice protoplast transformation.

Figure 34. Stable transformation of rice embryos with multigenic plasmids carrying nifH, nifM, nifU and nifS. 


\section{List of Tables}

Table 1. Maximum nitrogenase MoFe protein activity supported by $N$. benthamiana $\mathrm{NifH}$ and $A$.

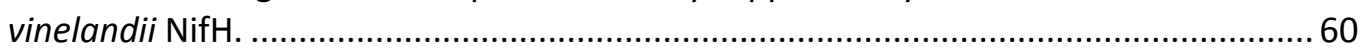




\section{List of Supplemental Material}

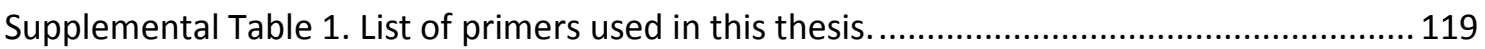

Supplemental Table 2. List of modular pieces generated in this thesis.................................. 127

Supplemental Table 3. List of assembled Transcriptional Units. ........................................... 131

Supplemental Table 4. List of assembled multigenic constructions. ........................................ 139

Supplemental Table 5. Optimization and motif settings used in Codon Optimization On-Line for tobacco nif synthetic designed genes. 142

Supplemental Table 6. Characteristics of yeast codon-optimized and tobacco synthetic designed

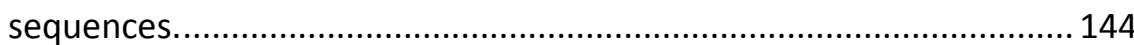

Supplemental Table 7. Summary of Chloroplast Transit Peptides studied in this thesis........... 145

Supplemental Table 8. Summary of processing products from TP-Nif fusions.......................... 147

Supplemental Table 9. Motif settings used for rice nif synthetic designed genes. .................... 148

Supplemental Table 10. Exclusion motifs present in codon-optimized nif genes. ..................... 150

Supplemental Table 11. Characteristics of nifH and nifM sequences regarding expression in rice. 151

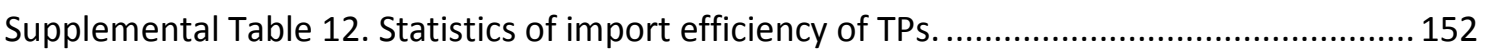

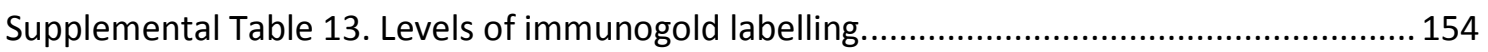

Supplemental Figure 1. Mass spectrometry results of bands present in $\mathrm{NifH}$ purification.......155

Supplemental Figure 2. Determination of $\mathrm{N}$-terminal sequence of TwinStrep-NifH using the standard Edman degradation method. .............................................. 159

Supplemental Figure 3. NifM and NifU co-elution of with Strep-tagged NifH.......................... 160

Supplemental Figure 4. Gene expression of TOC and TIC family members in A. thaliana and $O$. sativa.

Supplemental Figure 5. Biological context of $A$. thaliana genes containing selected Transit

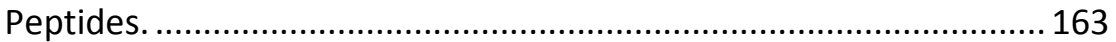

Supplemental Figure 6. Isolated protoplasts imaged using light microscopy. ........................... 164

Supplemental Figure 7. Immunogold Transmission Electron Microscopy images treated with polyclonal antibody. 165

Supplemental Figure 8. Confocal laser scanning microscopy images of wild-type and transgenic

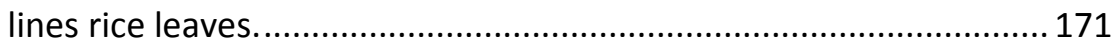

Supplemental Figure 9. Characteristics of $A$. thaliana and O. sativa Transit Peptides............. 173

Additional File 1. Sequences of genes used in this thesis. 174 


\section{Abbreviations}

2,4-D

AmpR

At

ADP

ATP

Av

BNF

bp

BSA

CAD

CAI

CaMV

CDS

CarbR

CIB

CFE

Col-0

COOL

CaMV

DBTL

DMSO

DNA

DTH

DTT

EDTA

eGFP

ETC

Fe-S

FW

GB

GC

GC3

h

HEPES
2,4-Dichlorophenoxyacetic acid

Ampicillin resistance

Arabidopsis thaliana

Adenosine diphosphate

Adenosine triphosphate

Azotobacter vinelandii

Biological Nitrogen Fixation

Base pair(s)

Bovine serum albumin

Computer assisted design

Codon adaptation index

Cauliflower Mosaic Virus

Coding sequence

Carbenicillin resistance

Chloroplast Isolation Buffer

Cell-free extract

Arabidopsis thaliana ecotype Columbia

Codon Optimization OnLine

Cauliflower Mosaic Virus

Design-build-test-learn

Dimethyl sulfoxide

Deoxyribonucleic acid

Sodium dithionite

1,4-dithiothreitol

Ethylene diamine tetraacetic acid

Enhanced Green Fluorescent Protein

Electron-transport chain

Iron-sulfur

Fresh weigh

GoldenBraid

Guanine-Cytosine content

Guanine-Cytosine content in the third base of codons

Hour

$\mathrm{N}$-(2-hydroxyethyl)-piperazine-N-(2-ethanesulfonic acid) 


\begin{tabular}{|c|c|}
\hline HPT & Hygromycin phosphotransferase \\
\hline HRP & Horseradish peroxidase \\
\hline ICU & Individual codon usage \\
\hline IME & Intron-mediated enhancement \\
\hline IPTG & Isopropyl $\beta$-D-1-thiogalactopyranoside \\
\hline ISC & Iron-Sulfur Cluster \\
\hline KanR & Kanamycin resistance \\
\hline $\mathrm{kb}$ & Kilobases \\
\hline $\mathrm{kDa}$ & Kilodalton \\
\hline $\mathrm{L}$ & Litre \\
\hline LB & Lysogeny Broth medium (also known as Luria-Bertani medium) \\
\hline MARs & Matrix attachment regions \\
\hline $\min$ & Minutes \\
\hline M & Molar \\
\hline MES & 2-(N-morpholino)-ethanesulfonic acid \\
\hline $\mathrm{mL}$ & Millilitre \\
\hline $\mathrm{mM}$ & Millimolar \\
\hline MoClo & Modular Cloning system \\
\hline MOE-PCR & Multiple Overlap Extension Polymerase Chain Reaction \\
\hline mRNA & Messenger RNA \\
\hline MS & Murashige and Skoog \\
\hline MSO & Osmoticum MS media \\
\hline MSP & Callus induction MS media \\
\hline MSR & Regeneration MS media \\
\hline MSS & Selection MS media \\
\hline MTS & Mitochondrial Target Signal \\
\hline $\mathrm{NaCl}$ & Sodium Chloride \\
\hline NADPH & Nicotinamide adenine dinucleotide phosphate \\
\hline $\mathrm{nt}$ & Nucleotide \\
\hline $\mathrm{Nb}$ & Nicotiana benthamiana \\
\hline $\mathrm{Nt}$ & Nicotiana tabacum \\
\hline $\mathrm{OD}_{600}$ & Optical Density measured at a wavelength of $600 \mathrm{~nm}$ \\
\hline OPR & Open Reading Frame \\
\hline Os & Oryza sativa (rice) \\
\hline $\mathrm{P}$ & Pellet \\
\hline PCR & Polymerase Chain Reaction \\
\hline pDNA & Plasmid DNA \\
\hline
\end{tabular}




\begin{tabular}{|c|c|}
\hline PEG & Polyethylene glycol \\
\hline PIC & Protease Inhibitor Cocktail \\
\hline PMSF & Phenylmethanesulfonylfluoride \\
\hline PTGS & Post-transcriptional gene silencing \\
\hline PVDF & Polyvinylidene difluoride \\
\hline qPCR & Quantitative Polymerase Chain Reaction \\
\hline RH & Relative Humidity \\
\hline RNA & Ribonucleic acid \\
\hline rRNA & Ribosomal RNA \\
\hline $\mathrm{rpm}$ & Revolutions per minute \\
\hline RT & Room temperature \\
\hline RT-qPCR & Reverse Transcription Quantitative Polymerase Chain Reaction \\
\hline $\mathrm{RuBisCO}$ & Ribulose-1,5-bisphosphates carboxylase/oxygenase \\
\hline $\mathrm{S}$ & Stroma \\
\hline s & Seconds \\
\hline SDS & Sodium dodecyl sulphate \\
\hline SDS-PAGE & Sodium dodecyl sulphate polyacrylamide gel electrophoresis \\
\hline SOB & Super Optimal Broth medium \\
\hline SOC & Super Optimal Broth medium with Catabolite repression \\
\hline SPP & Stromal processing peptidase \\
\hline SpecR & Spectinomycin resistance \\
\hline SUF & Sulfur mobilization pathway \\
\hline SynBio & Synthetic Biology \\
\hline TEV & Tobacco Etch Virus \\
\hline tGFP & Turbo Green Fluorescent Protein \\
\hline TGS & Transcriptional gene silencing \\
\hline TIC & Translocon at the inner envelope membrane of chloroplasts complex \\
\hline TOC & Translocon at the outer envelope membrane of chloroplasts complex \\
\hline ТP & Transit Peptide \\
\hline tRNA & Transfer RNA \\
\hline TU & Transcriptional Unit \\
\hline TMV & Tobacco mosaic virus \\
\hline $\mathrm{U}$ & Units \\
\hline UTR & Untranslated region \\
\hline WB & Western Blot \\
\hline WT & Wild type \\
\hline $\mathrm{X}-\mathrm{Gal}$ & 5-bromo-4-chloro-3-indolyl- $\beta$-D-galactopyranoside \\
\hline
\end{tabular}


Abbreviations

$\mu \mathrm{g}$

$\mu 1$

$\mu \mathrm{M}$
Microgram

Microliter

Micromolar 


\section{Resumen}

La biología sintética (SynBio), descrita como la aplicación de la ingeniería a la biología, tiene como objetivo desarrollar nuevos sistemas biológicos y modificar los existentes creando comportamientos predecibles.

La generación de cultivos agrícolas capaces de llevar a cabo la fijación biológica de nitrógeno, presentando bajos requerimientos de aporte de nitrógeno, se considera un desafío que podría conducir a una nueva revolución agrícola. Un posible enfoque para la generación de estos cultivos se basa en la transferencia directa de genes bacterianos nif, necesarios para la biogénesis de la nitrogenasa, al genoma de la planta. Los cloroplastos (plastidios) de plantas superiores son considerados compartimentos subcelulares que podrían albergar una enzima nitrogenasa activa, ya que exhiben las propiedades necesarias para acomodar el ensamblaje y la función de esta (poder reductor y abundancia de ATP) a pesar de la presencia de oxígeno. La modificación del genoma plastidial es posible en varios cultivos agrícolas, sin embargo, en cultivos de cereales esta técnica no es efectiva, lo que impone la necesidad de utilizar estrategias basadas en la expresión de transgenes codificados en el genoma nuclear, cuyas proteínas son posteriormente importadas a los plastidios.

En una primera parte, este trabajo presenta el uso de herramientas de biología sintética para optimizar la producción de la proteína de hierro de la nitrogenasa $(\mathrm{NifH})$ en cloroplastos de Nicotiana benthamiana. Los genes de fijación de nitrógeno de Azotobacter vinelandii nifH, $M, U$ y $S$ se rediseñaron para aumentar la acumulación de sus proteínas en células de tabaco, y su importación al cloroplasto se optimizó mediante la búsqueda de péptidos de tránsito de longitud mínima que funcionaran correctamente para cada proteína Nif específica. La presencia de la proteína NifM, una supuesta peptidilprolil cis-trans isomerasa, resultó ser necesaria para la obtención de proteína NifH soluble en el estroma de los cloroplastos. Es importante destacar que la proteína NifH purificada a partir de cloroplastos de tabaco fue activa en la reducción de acetileno a etileno, siempre que se co-expresara junto a NifU y NifS.

En una segunda parte, esta tesis describe la traslación de los conocimientos generados en experimentos con tabaco para su replicación en arroz. El mismo conjunto de genes de $A$. vinelandii se rediseñó para aumentar la acumulación de sus proteínas en Oryza sativa y los péptidos de tránsito, previamente seleccionados por su rendimiento en tabaco, se probaron para determinar su eficacia en la importación de una proteína marcadora a plastidios de diferentes tejidos de arroz, concretamente, callos, hojas y raíces. Los 
resultados mostraron que los péptidos de tránsito de longitud mínima de A. thaliana seleccionados también fueron capaces de mediar la importación de las proteínas Nif a cloroplastos de hojas de arroz. NifM, similar a lo que se ha observado anteriormente en bacterias, levadura y tabaco, resultó ser necesaria para la solubilidad de NifH en arroz. Sin embargo, no pudimos obtener plantas transgénicas de arroz que acumularan proteínas Nif en cloroplastos y, por lo tanto, la actividad de la proteína de hierro en cereales sigue siendo una incógnita.

En conjunto, esta tesis presenta una prueba de concepto de la idoneidad de los cloroplastos como orgánulos para albergar nitrogenasa, y un procedimiento de optimización para asegurar la expresión de cualquier transgen codificado en el núcleo dirigido a plastidios de $N$. benthamiana y $O$. sativa, centrándose en el diseño de versiones sintéticas de genes que confieren una mayor acumulación de proteína y caracterizando un conjunto de péptidos de tránsito que dirigen de manera eficiente sus proteínas transportadas mientras minimizan los aminoácidos remanentes en la proteína madura tras la importación. 


\section{Abstract}

Synthetic biology (SynBio), described as the engineering of biology, aims to develop new biological systems and to modify existing ones with predictable behaviours.

The generation of nitrogen-fixing crops with low nitrogen input requirements is considered a challenge that could lead to a new agricultural "green" revolution. A potential approach for their generation is the direct transfer of bacterial nif genes needed for the biogenesis of nitrogenase into the plant genome. Chloroplasts (plastids) of higher plants have been proposed as possible subcellular compartments in which to engineer active nitrogenase, as they have the necessary properties to accommodate the assembly and function of the nitrogenase enzyme (reducing power and ATP abundance), despite their oxygenic environment. Chloroplast genome engineering has been achieved in several major crops. However, cereals remain elusive to this technique, imposing the need of using strategies that rely on the expression of nucleus-encoded transgenes with proteins being subsequently imported into plastids.

The first part of this work reports the use of SynBio tools to optimize the production of nitrogenase Fe protein (NifH) in the chloroplasts of Nicotiana benthamiana plants. Azotobacter vinelandii nitrogen fixation genes nifH, $M, U$, and $S$ were re-designed for protein accumulation in tobacco cells, and targeting to the chloroplast was optimized by screening minimal length transit peptides performing properly for each specific Nif protein. Putative peptidyl-prolyl cis-trans isomerase NifM proved necessary for NifH solubility in the stroma. Importantly, NifH purified from tobacco chloroplasts was active in the reduction of acetylene to ethylene, with the requirement of NifU and NifS coexpression.

In the second part, this thesis describes the translation of the lessons learned in tobacco to rice. The same set of $A$. vinelandii genes were re-designed for protein accumulation in Oryza sativa and the transit peptides selected for their performance in tobacco were tested for their efficiency in the import of a nuclear-encoded marker protein into plastids of different rice tissues, namely, callus, leaf, and root. Results showed that the A. thaliana minimal length transit peptides selected were also able to mediate Nif protein import into rice leaf chloroplasts. NifM, similar to what has been observed previously in bacteria, yeast and tobacco, proved necessary for NifH solubility in rice. However, we were unable to obtain rice transgenic plants accumulating any Nif protein in chloroplasts and thus, nitrogenase Fe protein activity in cereals remains elusive. 
Altogether, this thesis presents a proof of concept of the suitability of chloroplasts as organelles to host nitrogenase, and an optimization pipeline to engineer the reliable expression of any nuclear-encoded transgenes in $N$. benthamiana and $O$. sativa plastids, focusing on designing synthetic gene versions that confer higher protein accumulation and characterizing a set of chloroplast transit peptides that efficiently target their cargo proteins while minimizing scar amino acids in the mature protein after cleavage. 


\section{Introduction}

\section{Synthetic Biology and plants}

\subsection{Introduction to Synthetic Biology}

Synthetic Biology (SynBio) is a new discipline that emerged in the decade of the 2000s as a confluence of biology and engineering. SynBio can be defined as a combination of DNA technology, engineering principles, and computational tools applied to the modification of natural systems or the creation of artificial ones to exhibit predictable behaviours (Andrianantoandro et al., 2006; Purnick and Weiss, 2009; Schwille, 2011).

A hallmark of SynBio is the use of the foundational engineering principles of decoupling, abstraction and standardization, to make the design of biological systems more efficient and scalable (Endy, 2005). Decoupling allows simplification of complex problems into smaller and simpler ones, that can be addressed individually. Abstraction facilitates the management of complexity by identifying and establishing hierarchies of functional levels for the design process. Standardization is used to define and characterize functionally equivalent and interchangeable (i.e. orthogonal) parts for construction and testing (Endy, 2005).

Two main approaches in the development of a SynBio process can be distinguished, consisting in either top-down or bottom-up assembly. The first is an application-driven strategy, which starts from an existing natural system that is modified with the goal to construct synthetic compositions with predictable and controllable properties. On the other hand, the second is a knowledge-driven approach, based on the de novo design of biological systems with properties not previously found in nature (Ausländer et al., 2017; Scharff and Bock, 2014). Engineering Azotobacter vinelandii nitrogenase Fe protein biosynthesis in plastids, the object of study of this doctoral thesis, consists in a top-down approach, as it aims to build a new biological function into an existing organelle using Nicotiana benthamiana and Oryza sativa as chassis.

The set of steps we have taken to construct such a system are defined in the design-buildtest-learn (DBTL) cycle (Figure 1). As its name states, it is a workflow that encompasses four main stages: design, build, test and learn. The first step defines the problem, objectives, expected outputs and possible constraints. Often, it is not feasible to build all the proposed solutions to the specified objective, therefore computer-aided design (CAD) tools can help to optimize the selection of parameters, choosing the most favourable design based on previous knowledge. The 'build' stage involves the fabrication and assembly of parts. The 'test' stage encompasses the integration into a host and 
experimental analysis of the synthetic systems. Then, the data regarding the fitness of the solution is analysed at the 'learn' stage and applied back to the design stage, modifying it accordingly (Liu et al., 2013; Slusarczyk et al., 2012).

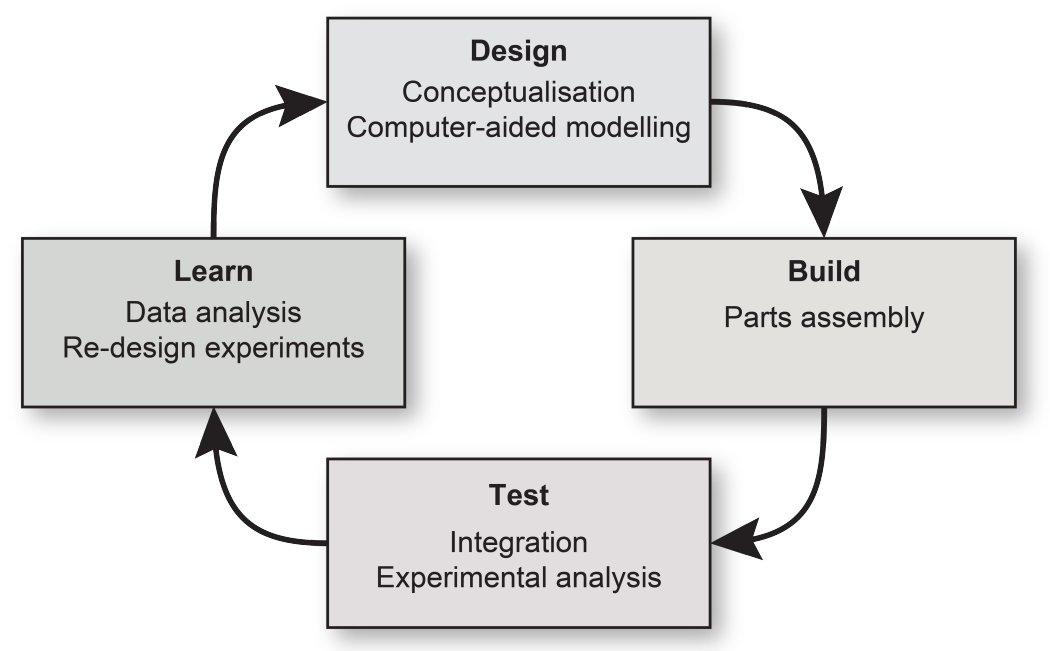

Figure 1. The design-build-test-learn (DBTL) cycle in synthetic biology.

Schematic representation of the cyclical synthetic biology workflow for engineering living organisms. Adapted from Boeing et al. (2016).

The application of the SynBio approach to microbial systems has tremendously impacted basic life science research, human health, environmental protection, and even economic growth in recent years (Meng and Ellis, 2020). Some hallmark examples of it are the rewriting of Escherichia coli genes essential for growth that require synthetic amino acids that do not exist in nature (Rovner et al., 2015), the redesign of a minimal Mycoplasma mycoides genome (Hutchison et al., 2016), the synthesis of five additional chromosomes in the synthetic yeast project (Sc2.0) (Richardson et al., 2017), the reprogramming of the genetic code of $E$. coli to reduce the number of codons used by the host (Fredens et al., 2019) or the engineering of carbon fixation into E. coli (Gleizer et al., 2019). Furthermore, microbes, especially yeast, have become a useful chassis in metabolic engineering for the biosynthesis of several high-value compounds demanded in the pharmaceutical industry such as the antimalarial artemisinin (Paddon et al., 2013), the opioids thebaine and hydrocodone (Galanie et al., 2015) or the cannabinoids tetrahydrocannabinol and cannabidiol (Luo et al., 2019), to cite some examples.

Microbial systems are easy and fast to engineer; however, their unicellular nature poses certain limitations, reason why synthetic biology has advanced to multicellular systems, remarkably to plants (Liu and Stewart, 2015). 


\subsection{Plant synthetic biology}

Plants are a fundamental part of human life and society for several reasons. They are the most important source of primary metabolites (i.e. sugar, lipids and proteins) into the food chain, as well as a source of energy, raw materials and last but not least, oxygen. Their relatively slow growth, genomic complexity and lack of available tools for genetic manipulation, compared to that of bacteria and yeast, have delayed the development of plant SynBio projects (Boehm and Bock, 2019). However, from a scientific point of view, plants are an appealing chassis since they have been engineered for more than 50 years using recombinant DNA technology and also due to their ability to perform photosynthesis, their extensive secondary metabolism with products valuable for industry, and their large-scale, rapid and relatively low-cost cultivation (Boehm et al., 2017).

Several species are being engineered in the present, chosen either for their ease of genetic modification or their importance in food production. Two dicotyledonous species from the first group, Arabidopsis thaliana and N. benthamiana, and one monocotyledonous from the second, O. sativa, are among the most popular chassis used in plant SynBio approaches and the ones used in this work. Arabidopsis is popular due to its little stature, short life-cycle, small well-annotated genome and amount of genetic tools available, furthermore, its research is easily transferable to Camelina sativa valued for oilseed production (Flavell, 2009; Holland and Jez, 2018; Stewart et al., 2018). N. benthamiana prominence lies in the ease of its transformation and its ability to rapidly and highly express transgenes, making it the plant biofactory of choice (Bally et al., 2018). In the monocotyledonous group, while Zea mays hoards most of the GM crop approvals (ISAAA, 2020), O. sativa is an appealing chassis as it is the staple crop responsible for providing one-fifth of the calories consumed worldwide (Sharif et al., 2014).

Pioneering plant SynBio projects have been aimed at the generation of plants that offer solutions to our needs of food, water, materials, and energy. Engineered plants are being developed today to help us secure food yields and achieve sustainable agriculture by improving photosynthesis (Batista-Silva et al., 2020), engineering disease resistance (Dong and Ronald, 2019) or enabling biological nitrogen fixation in crops (Bloch et al., 2020; Burén and Rubio, 2018). Other successful projects include the biofortification of crops such as rice, maize or bananas to confront malnutrition from micronutrient deficiencies (Bouis and Saltzman, 2017; Van Der Straeten et al., 2020) and the removal of plant-derived allergens in wheat, among other food crops (Sánchez-León et al., 2018; Steinwand and Ronald, 2020). 
The use of plants as biofactories offers an alternative for efficient and inexpensive expression of foreign proteins with low biosafety concerns. A great variety of compounds such as biopharmaceuticals proteins, antibodies or vaccine antigens are routinely manufactured using these technologies, including a candidate vaccine against the SARS$\mathrm{CoV}-2$ virus which at the moment of writing this dissertation was being evaluated in a Phase I clinical trial (Mortimer, 2019; Shanmugaraj et al., 2020; Ward et al., 2020). Moreover, the fibres that compose plants are a valuable element in the production of biofuels, which can be greatly improved by engineering their properties (Mortimer, 2019).

And lastly, plants are also being currently engineered to fight environmental pollution derived from human activities by augmenting the phytoremediation capabilities of certain species (Yan et al., 2020) and implementing biosensors in plants to detect toxins and contaminants (Verma and Rani, 2020).

Although public opinion supports the use of microbial SynBio for industrial applications and the production of biopharmaceuticals, this is not the case for genetically engineered plants, particularly food crops (Cui and Shoemaker, 2018). Given the current situation regarding the heavy regulation of genetically engineered plants, especially in the EU, the real ability of SynBio to impact on problems such as sustainable agriculture and food security could be hampered if the regulatory framework is not changed (Guidance for risk assessment of food and feed from genetically modified plants, 2011; Nicolia et al., 2014).

\subsection{The DBTL process in plant SynBio}

The different stages of the cycle will be discussed in more detail as follows, with attention to plant-specific SynBio idiosyncrasies.

\subsubsection{Design}

In the design of a synthetic system, two sets of factors need to be considered: outside features, such as the transformation method, the host organism and the location of protein accumulation, and inside features, such as those which affect the transcription of your gene(s) of interest and the translation of its(theirs) mRNA product(s) (Figures 2 and 3) (Ullrich et al., 2015).

\subsubsection{Outside parameters to optimize during design}

Outside features choices such as transformation method, host system or location of protein accumulation, will depend on the final goal of the design, e.g. production of a recombinant protein for purification, biofortification of a crop or introduction of a 
completely new pathway (Figure 2). The choice is to an extent bound to the requirements of the engineered trait, however, the tools used to enable those factors can be optimized.
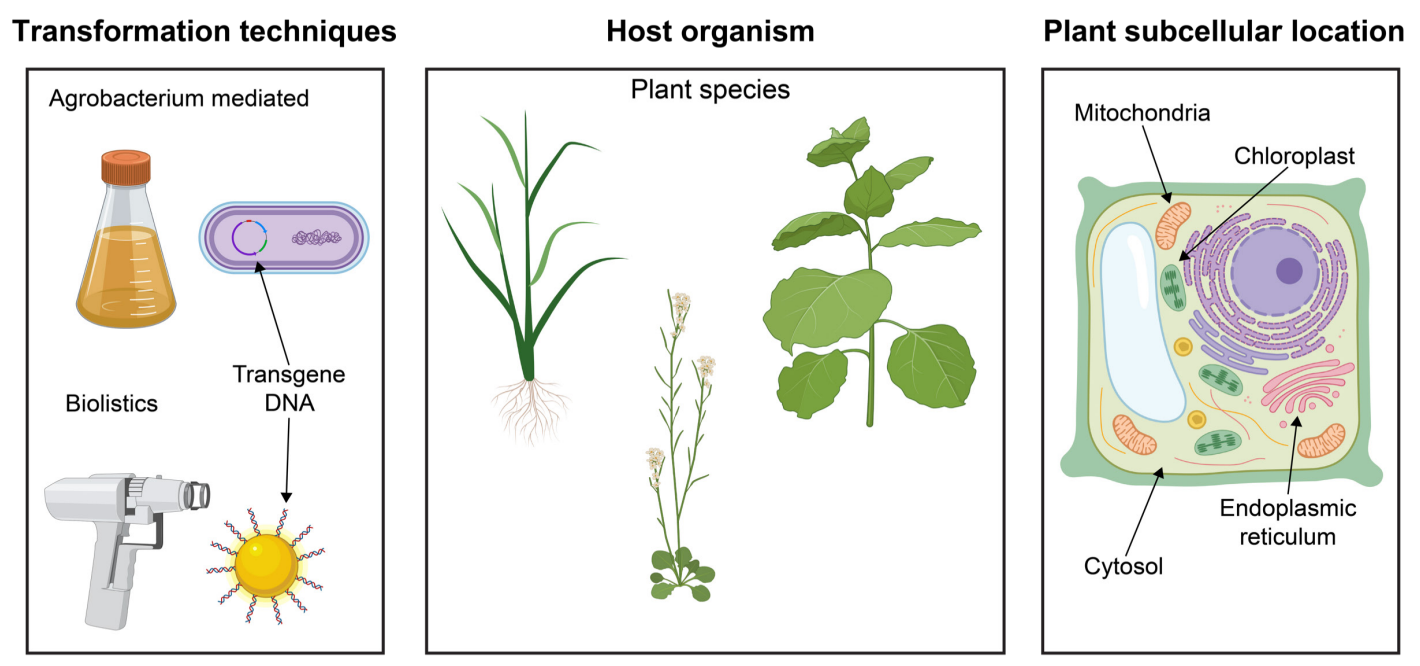

Figure 2. Schematic overview of outside features affecting synthetic gene expression.

Outside features are those such as host system, subcellular location of the expressed protein and transformation method used. Adapted from Egelkrout et al. (2012) and Ullrich et al. (2015). Created with BioRender.com (2020).

Regarding the transformation method, foreign DNA can be introduced into plant cells by physical or biological methods. Common physical methods are particle bombardment, PEG-mediated delivery, or electroporation, which are relatively simple and can be applied to a wide range of species (Barampuram and Zhang, 2011). Biological methods mainly rely on Agrobacterium spp. bacteria carrying the recombinant DNA cloned into the T-region of binary plasmids. This method enables largely intact integration of a synthetic DNA into the chromosome and, depending on the strain used, the number of copies can be controlled (Tzfira and Citovsky, 2006). However, species that remain recalcitrant to Agrobacterium transformation still rely on physical methods (Hiei et al., 2014). The choice of transformation method will influence the plasmid used for construction of the synthetic DNA since physical methods tend to prefer high-copy lowsize backbones and biological methods based on Agrobacterium require the DNA to be integrated to be flanked by the left and right border sequences (Barampuram and Zhang, 2011).

Concerning host systems, $A$. thaliana is the most popular choice for a plant chassis, given its long laboratory history and resources available for the metabolic engineer and synthetic biologist (Holland and Jez, 2018). Other systems include dicotyledonous platforms, e.g. tobacco, which stand out for their results (Stewart et al., 2018). Monocots, such as maize or rice, sometimes are the final target of the modifications engineered in model plants, but they present challenges in their manipulation that force them into a 
second-place as plant chassis (Sood et al., 2011). The host choice will impact on the modules, e.g. promoters, terminators or selection markers, that will be required for the expression of the recombinant DNA.

Regarding protein accumulation location, specific projects might include requirements to import recombinant proteins into certain subcellular organelles. By default, nuclearencoded proteins synthesised by cytosolic ribosomes accumulate in the cytosol of plant cells. Only when an appropriate peptide signal is included in the transgene sequence, proteins migrate to specific organelles post-translationally (Benchabane et al., 2008). A range of peptide signals exist in order to direct a protein to the desired destination organelle, including N-terminal mitochondrial signal peptides (MTP); plastid transit peptides (TP); signal peptides of the secretory pathway (SP) that direct proteins to the endoplasmic reticulum further heading to the apoplast or vacuole; the C-terminal peroxisome target sequence (SKL) and the nuclear localization signals (NLS) (Benchabane et al., 2008). In all cases, the signals used need to be optimized to maximize their organelle targeting efficiency and specificity, while minimizing the residues left in the mature protein in the case of import into organelles where there is posterior signal cleavage.

\subsubsection{Inside parameters to optimize during design}

Building up new capabilities in an organism relies most of the time on the expression of newly introduced transgenes. Inside factors that can be optimized are those that affect the transcription of the genes of interest and the translation of their mRNA products (Figure $3)$.

The temporal and spatial expression of a transgene is controlled by the choice of its driving promoter. Promoter selection is an especially critical variable when expressing multiple transcriptional units since repeated use of the same promoter can trigger transcriptional gene silencing (TGS) (Vaucheret and Fagard, 2001). Additionally, terminators have also been described to be of high importance in the control of transgene expression level, by controlling the $3^{\prime}$ end processing of mRNAs (Carswell and Alwine, 1989; Ingelbrecht et al., 1989), and their stability as targets of post-transcriptional gene silencing (de Felippes et al., 2020; Pérez-González and Caro, 2018). Fortunately, libraries of promoters originating from plants and their pathogens either constitutive, inducible or tissue-specific, as well as libraries of terminators also from plants and their pathogens, have been characterized, providing information for their selection depending on specific projects' necessities (Engler et al., 2014; Feike et al., 2019; Sarrion-Perdigones et al., 2013; Vazquez-Vilar et al., 2017). 


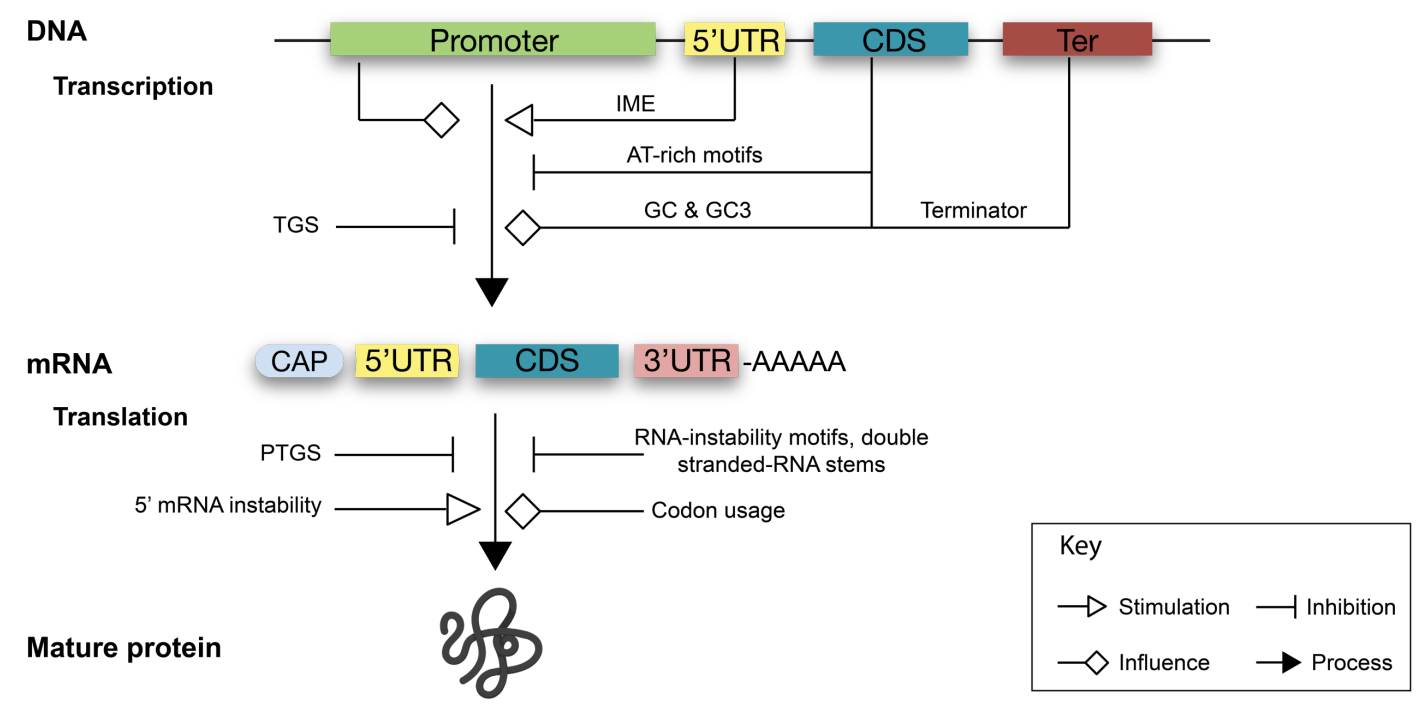

Figure 3. Schematic overview of inside features affecting synthetic gene expression.

Inside features affecting the transcription of a gene are promoters, terminators, introns mediating enhancement (IME) in the 5' Untranslated Region (5'UTR), GC and GC3 content, motifs present in the coding sequence (CDS), and the activation of transcriptional gene silencing (TGS). Those affecting translation of its mRNA product are 5' mRNA instability, presence of RNA-instability motifs and double-stranded structures, the codon usage and the activation of post-transcriptional gene silencing (PTGS). Adapted from Egelkrout et al. (2012) and Ullrich et al. (2015).

Furthermore, transgene expression can be improved by the addition of certain introns in the 5' untranslated region (UTR) by the mechanism known as intron-mediated enhancement, i.e. the effect of an intron only when downstream of, and close to, the start of the gene, that generates an increase in mRNA accumulation (Rose, 2008, 2004). Increases of 10-fold or more in expression levels have been reported by this technique, with some introns being routinely used, e.g. the maize ubiquitin 1 intron or the rice TubAl (Gallegos and Rose, 2015; Pan et al., 2016).

In the case of the transgene sequence, certain level of edition is possible so that the synthetic nucleotide sequence encodes the same product as the gene of interest while optimizing its transcription and translation by adapting codon usage, tweaking GC content and removing deleterious motifs (Jackson et al., 2014).

Codon usage bias refers to the frequency of use of synonymous codons in the coding DNA of a certain species. The codon usage of an organism can be measured with indexes such as the codon adaptation index (CAI), representing how often the most frequent codon for each amino acid is used in a gene (Sharp and Li, 1987), or the individual codon usage (ICU), representing how closely a sequence matches the codon usage pattern of the host (Chung and Lee, 2012). The rationale behind codon optimization is to mimic the endogenous codon usage, so the host tRNAs supply will be used in balance with the host 
transcriptome, and to remove rare codons that could cause translational pausing (Makrides, 1996).

Regarding GC content, there has been some controversy as to what is the optimal value in coding sequences to be expressed in a heterologous system. It is a common practice in synthetic gene design to match the GC content of a transgene to that of the host organism's coding sequences (Gustafsson et al., 2012). However, a recent study showed that, after stable transformation, synthetic genes with plant-comparable GC content failed to produce the anticipated high yield of protein production and undertook rapid and stable transposon-like TGS. On the contrary, microalgae native genes harbouring high GC content, particularly in the third position of translational triplets (GC3), displayed enhanced protein accumulation and transgenerational expression stability (Sidorenko et al., 2017). This is in accordance with transposons, a major target for gene silencing, having a clear distribution preference for AT-rich sequences (Le et al., 2000), but the mechanism regulating it is still unknown.

Concerning the exclusion of motifs considered to be deleterious for heterologous protein accumulation, the removal of cryptic intron splice sites and RNA instability motifs has been described to increase transcription and enhance translation in plants (Gutiérrez et al., 1999; Jackson et al., 2014). Furthermore, sequences causing premature polyadenylation sites have previously been implicated in poor expression (Haffani et al., 2000; Mathew et al., 2011).

Translation of mRNA into a mature protein is the second key step for protein accumulation. An important factor that can be optimized here to increase heterologous protein production is $5^{\prime} \mathrm{mRNA}$ stability. The secondary structure of mRNA has great importance in translation efficiency. If the 5'-end folds into stable secondary structures, it can reduce translational initiation (Kozak, 1986), therefore it is always advisable to keep the local secondary structure as "open" as possible within the first $50 \mathrm{nt}$ of the open reading frame (ORF) to aid translational initiation (Jackson et al., 2014). Also, motifs generating double stranded-RNA stems of $12 \mathrm{bp}$ or more in any part of the ORF should be avoided to prevent translational stalling (Chou and Moyle, 2014).

Translation can be repressed by post-transcriptional gene silencing (PTGS) triggered as the consequence of excessive aberrant transcript levels (Que et al., 1997). High expression of transgenes can be counter-productive if it leads to saturation of the RNA maturation machinery causing the production of unproperly matured transcripts, thus the considerations regarding promoter and terminator use are of the uttermost importance for translation as well. 


\subsubsection{Computational tools for design}

The use of CAD tools allows the synthetic biologist to optimize design parameters and test possible designs in silico before construction (Liu and Stewart, 2015). Gene synthetic design has gained popularity in the last years, due in part to the advances in DNA synthesis, giving birth to an extent of CAD tools that streamline the design of longer and more complex DNA sequences (Gould et al., 2014; Webster et al., 2017). Nevertheless, the predictive power from algorithms of the behaviours encoded in DNA sequences is still limited and iterations of the DBTL process are necessary to achieve the optimal sequence.

Codon Optimization OnLine (COOL) (Chin et al., 2014) is one of the most complete CAD tools available for synthetic gene design. COOL offers a multi-objective optimization using metaheuristics, considering several user-defined parameters ranging from codon usage and GC content, to motifs and repeats removal and 5' RNA folding instability assessment (Figure 4). These parameters are the key elements of the design and need to be established specifically for each project from a thorough literature survey on the host organism of choice. The software provides various solutions, leaving the user the ultimate choice of the sequence that offers the best compromise between all the design criteria considered (Chin et al., 2014).

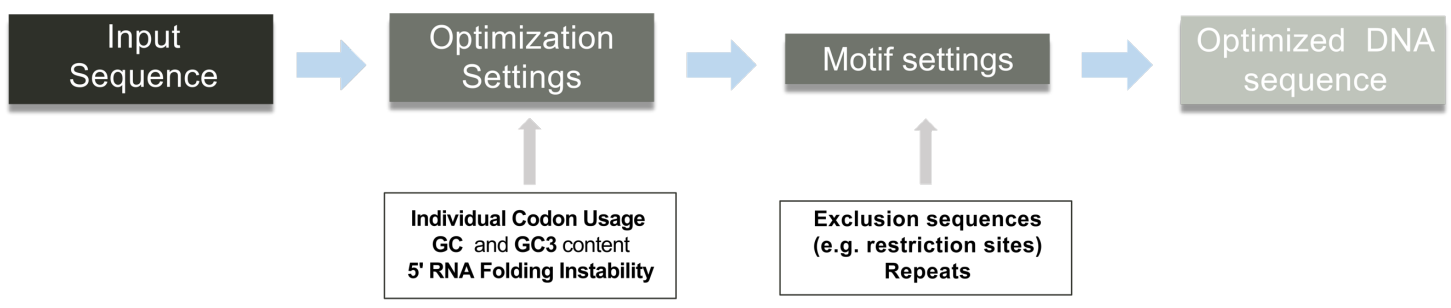

Figure 4. Workflow followed in Codon Optimization OnLine.

Optimization settings as individual codon usage, which measures the frequency by which individual codons appear in a set of coding sequences, GC and GC3 content and 5' RNA folding instability were used alongside with the removal of motifs such as polyadenylation sequences, RNA instability motifs and repeats, providing a multi-objective optimization for synthetic gene design. Adapted from $\mathrm{Yu}$ et al. (2017).

\subsubsection{Build}

An important task within a SynBio project is the building of new or redesigned versions of DNA constructs. Traditionally, they were made using relatively time-consuming DNA manipulation protocols that limited the scope of research projects, e.g. DNA digestion using one or two restriction enzymes, dephosphorylation of vector ends or use of multiple-cloning sites for the insertion of a single DNA fragment into a vector. Although still used today, more efficient methods enable parallel directional assembly of multiple 
parts of DNA in a single reaction, enabling the fast and easy generation of complex devices.

\subsubsection{Modular cloning tools}

Two critical factors that have changed the way new constructs are built are standardization of basic parts, allowing their reuse and exchange between users, and the development of modular assembly strategies, allowing the efficient association of these standard parts into more complex arrangements (Werner et al., 2012).

The BioBrick standard was the first widely-adopted modular assembly method (Knight, 2003). Its key feature is idempotency, as BioBricks parts can be assembled resulting in new BioBricks modules that retain the same structure as the original parts regarding the flanking restriction sites (Knight, 2003; Shetty et al., 2008). However, BioBrick assembly relies on the use of type II restriction enzymes, meaning that the two assembled parts are separated by an eight bp scar sequence and that the process is limited to the association of only two parts in a single step.

To overcome these constraints, new modular assembly systems have been developed in the last decade, such as Golden Gate (Engler et al., 2009) which resulted in GoldenBraid (GB) (Sarrion-Perdigones et al., 2011) and Modular Cloning (MoClo) (Werner et al., 2012) assembly methods for plant SynBio. Golden Gate uses Type IIS restriction enzymes, capable of recognizing nonpalindromic sequence motifs and cleaving outside of their recognition site, resulting in custom-designed DNA overhangs. These overhangs enable parallel directional assembly of multiple parts using only one restriction enzyme and DNA ligase in a one-pot, one-step reaction (Engler et al., 2009). Both GB and MoClo assembly methods have gained considerable popularity and several kits have been developed containing tools and parts for plant engineering (Emami et al., 2013; Engler et al., 2014; Lin et al., 2014; Sarrion-Perdigones et al., 2013; Vazquez-Vilar et al., 2016, 2017).

In GB and MoClo assembly systems, the notion of genetic elements, e.g. promoter or coding sequence, has been converted into standard parts with positions defined by four bp codes flanking them, known as Level 0 parts or Phytobricks, and a common syntax for these codes has been agreed globally to enable their exchange between laboratories (Patron et al., 2015). 


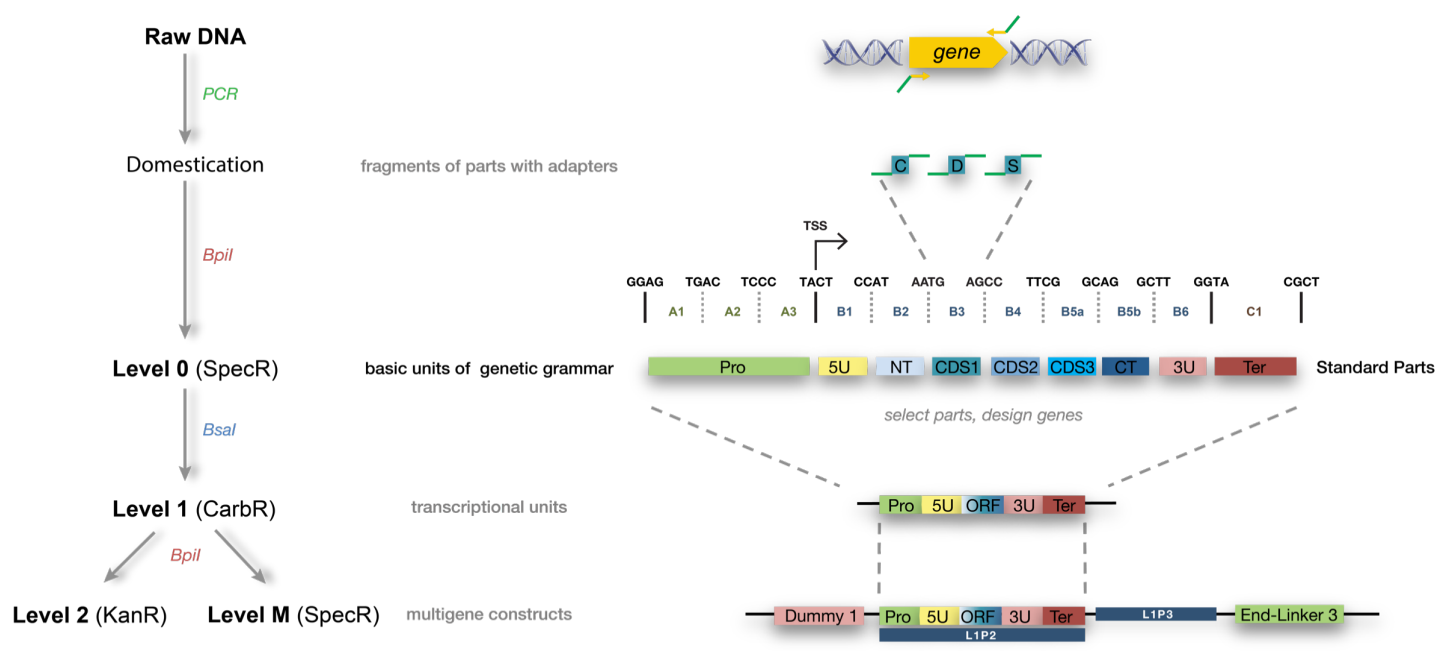

Figure 5. Overview of Golden Gate MoClo Assembly Standard.

Standard (Level 0) parts are assembled through a BpiI-mediated restriction-ligation reaction from single or multiple PCR-amplified sequences with primers that contain the corresponding four bp adapters and BpiI restriction sites. The common syntax for plants defines the four base-pair overhangs that join standard parts which will determine the position of each piece within the future transcriptional unit (TU), enabling parts to span multiple fusion sites. Level 0 parts are assembled into Level 1 acceptor backbones to make complete TUs via a BsaI-mediated restriction-ligation reaction. The Level 1 acceptor chosen in the assembly will determine the position of that TU in the multigene assembly. Subsequently, up to six Level 1 TUs can be can assembled in an orderly manner in a Level 2 or M acceptor backbones through a Bpil-mediated restriction-ligation reaction. Any Level 1 transcriptional unit can be substituted for a dummy piece and the correct end-linker must be used to join the last Level 1 unit to the acceptor. CarbR: carbenicillin resistance; KanR: kanamycin resistance; SpecR: spectinomycin resistance. Adapted from Engler et al. (2014).

The MoClo system contains three levels of hierarchy (Level 0,1 , or 2 ) which can be used in successive assembly steps (Figure 5) (Werner et al., 2012). Basic genetics parts are PCR amplified from a DNA source (genomic DNA, cDNA or plasmids) using primers containing a four nt code and BpiI site, and cloned into a Level 0 universal vector. Level 0 standard parts are directionally assembled in a transcriptional unit (TU) via BsaI mediated restriction-ligation into Level 1 acceptor vectors. There are six different Level 1 positions available, which define the location of each TU in a multigene construct. Subsequently, MoClo allows the concurrent assembly up to six TUs via BpiI mediated restriction-ligation into acceptor vectors as Level 2, $\mathrm{M}$ or $\mathrm{P}$, comprising the highest hierarchy level. Furthermore, the use of openable "end-linkers" and a combination of Bsal-BpiI enzymes allows an iterative process to generate Level 2 constructs larger than six TUs, enabling the creation of more complex circuits.

The implementation of these assembly methods has enabled plant SynBio, leading to the rapid generation of constructs efficiently and with high accuracy, facilitating their prototyping, testing, and iteration. 


\subsubsection{Computational tools}

Due to the number of standard parts generated and the increasing complexity of the SynBio systems developed, it is necessary to deposit these parts along with their associated information in bioregistries, so they can be accessed and shared. Some wellknown non-commercial bioregistries for Golden Gate standard parts are GB's (https://gbcloning.upv.es) or AddGene (https://www.addgene.com), which also serve as biorepositories. Tools for the management and building of parts and devices have also been developed by research groups, e.g. MoClo planner (Shaer et al., 2013) or ePARTS (Pardo and Caro, 2017), and private companies e.g. Geneious (Kearse et al., 2012) or Benchling (https://www.benchling.com/), allowing users to streamline their experiments.

\subsubsection{Test}

With the 'design' and 'build' of synthetic DNA modules becoming efficient and cheap, the bottleneck of the DBTL cycle for bioengineering is the limited number of screening tools available to rapidly test a large number of constructs (Pouvreau et al., 2018). However, plants have a prominent advantage as they can be transiently or stably transformed, and transient expression platforms have the potential to break this bottleneck.

\subsubsection{Transient expression}

Transient expression assays, in which, after DNA introduction into plant cells, the transgene undergoes rapid and high transcription and translation without integrating into the genome, are particularly useful to rapidly perform tests on constructs and to obtain high amounts of recombinant protein in a short period of time while avoiding possible harmful long-term effects on the plant cell.

This rapid and high-expression can be achieved by delivering DNA using Agrobacterium or physical methods, as well as by a hybrid strategy based on integrating transgenes into complete or deconstructed genomes of viruses, which are then delivered to plant cells (Barampuram and Zhang, 2011; Naseri et al., 2019).

Several techniques have been developed over the years, being agroinfiltration, magnifection and protoplast transformation the most commonly used. Agroinfiltration is a method based on the introduction of a suspension of Agrobacterium bacteria carrying the recombinant DNA into a plant leaf, typically $N$. benthamiana, by direct injection or by vacuum infiltration, generating an infection of plant cells that express the transgene. It is a common procedure to co-infiltrate with the silencing suppressor P19 from the plant 
pathogenic tomato bushy stunt virus to avoid PTGS and boost the expression of the transgenes of interest (Csorba et al., 2015; Li, 2011; Muthamilselvan et al., 2019). Conversely, magnifection uses the agroinfiltration procedure to deliver viral replicons together with the transgene of interest to plant cells, achieving an even higher expression yield (Gleba et al., 2005).

Protoplasts consist of plant cells in which the cell wall has been previously digested. They are a proven versatile cell-based experimental system widely used in functional characterization of genes and protein analysis in several species, e.g. Arabidopsis, maize, rice or tobacco (Zhang et al., 2011). The lack of the cell wall allows for molecules like DNA and proteins to be delivered using physical methods as PEG-calcium transfection or electroporation. After entering the cell, transgenes are then rapidly and transiently expressed. Protoplast regeneration into stably transformed plants is also possible when cultured in specialized plant growth conditions and sterile culture media (Damm et al., 1989).

\subsubsection{Stable expression}

Stable transformation involves the integration of the introduced DNA into the plant genome, which is necessary to produce transgenic plants with heritable traits. In this approach, a marker gene is commonly integrated along with the genes of interest in order to detect positive transformants. However, both Agrobacterium-mediated transformation and biolistic methods lack control over the site, frequency and structure of exogenous DNA integration, which leads to time-consuming screenings in search of lines that express transgenes stably and in high levels (Butaye et al., 2005; Pouvreau et al., 2018).

Some stable transformation methods, such as floral dipping, directly transform female gametes (Clough and Bent, 1998), however, many species are not amenable to this procedure and rely on somatic cell transformation, which requires extensive tissue culture. The bottleneck of stable engineering lays in the step of plant regeneration from transformed tissues, as it involves long tissue culture periods with conditions that have to be set experimentally for each species (Altpeter et al., 2016). This weakness limits the possibility to test large numbers of constructions in stably transformed plants of most crops (Pouvreau et al., 2018).

\subsubsection{Learn}

The DBTL cycle ends at the 'learn' stage, in which all data produced in previous steps are analysed and combined to refine the original hypothesis, prompting a new one that 
Introduction

will be tested in a new cycle iteration (Pouvreau et al., 2018). The process is then repeated until the target function is achieved, enabling a systematic design of biological systems. 


\section{Application of SynBio tools to the engineering of Biological Nitrogen Fixation in plants}

\subsection{The worldwide need for nitrogen}

Cereal crops, mainly rice, wheat and maize, provide half of the world's daily calories consumed directly by humans (Awika, 2011). One of the crucial factors for crop productivity is nitrogen availability to plants (Erisman et al., 2008). Since major staple cereal crops are unable to form symbioses with nitrogen-fixing bacteria, as occurs in legumes, the nitrogen needed for higher yields is supplied via synthetic nitrogen fertilizers. Ammonia fertilizer is industrially obtained by the Haber-Bosch process, in which molecular nitrogen gas is mixed with hydrogen at very high pressure (300 atm) and temperature $\left(500^{\circ} \mathrm{C}\right)$ over a metal catalyst (Gilchrist and Benjamin, 2017).

The use of synthetic nitrogen fertilizers has increased steadily since the 1960s (Stewart and Roberts, 2012). Despite the food-security benefits offered by synthetic fertilizers since then, their industrial production and extensive use present environmental and sociopolitical issues (Erisman and Sutton, 2008). The Haber-Bosch process consumes vast amounts of non-renewable fossil fuels, increasing the atmospheric carbon dioxide pollution. Besides, inefficient assimilation into crops and excess application of synthetic fertilizers lead to leaks into the environment, resulting in water eutrophication (e.g. cyanobacterial blooms) and air pollution (e.g. nitrous oxide, a greenhouse gas) (Erisman et al., 2015). Furthermore, the high prices these fertilizers reach makes them inaccessible to many smallholder farmers in developing regions (Mueller et al., 2012).

\subsection{Biological nitrogen fixation as an alternative}

Biological nitrogen fixation (BNF) is the conversion of dinitrogen $\left(\mathrm{N}_{2}\right)$ into ammonia $\left(\mathrm{NH}_{3}\right)$ catalysed by a nitrogenase enzyme. Although this is an essential process that supports life on Earth, only a small group of bacteria and archaea known as diazotrophs (dinitrogen eaters) are able to fix nitrogen on their own (Zehr, 2013), accounting for about two-thirds of the world's total fixed nitrogen (Canfield et al., 2010).

BNF has been envisioned as an alternative for providing biologically active nitrogen to cereal crops since the 1970s, as Normal Borlaug stated in his Nobel Prize lecture (Borlaug, 1972). Great advances in understanding nitrogenase biosynthesis and function followed, such as the functional transfer of nitrogen fixation genes from Klebsiella oxytoca to E. coli (Dixon and Postgate, 1972). Since then, technical developments and the birth of SynBio have spiked the interest in engineering BNF in cereals to sustain cereal 
crop productivity. Three different strategies have been proposed to achieve this goal (Figure 6).

The first strategy mainly relies on enhancing nitrogen-fixing capabilities of bacteria that are naturally associated with cereals, either residing on or near the root surface (epiphytes) or within plant cells (endophytes) (Mus et al., 2016). It is considered the approach with lower technical barriers (Bloch et al., 2020). However, although inoculation of crops can improve growth and yield, the positive results obtained in greenhouse studies do not always correlate with the performance of these bioinoculants in the field (Compant et al., 2019). Nevertheless, Ryu and colleagues recently engineered inducible nitrogenase expression, under the control of signals such as root exudates or phytohormones, in microorganisms associated with cereals. They demonstrated nitrogenase activity equivalent to the native diazotrophs in engineered variants of the endophyte Azorhizobium caulinodans and the epiphyte Pseudomonas protegens (Ryu et al., 2020).

The second strategy focuses on engineering new associations between cereals and nitrogen-fixing bacteria to recreate nodule-forming symbiosis (Rogers and Oldroyd, 2014). Even though similarities have been found between the Nod factors, responsible for legume-rhizobia symbiosis, and the Myc factors, involved in arbuscular mycorrhizal symbiosis in cereals, strategies leading to the infection and nodule formation in cereals are to this day elusive (Rogers and Oldroyd, 2014).

The third strategy aims to generate varieties of cereals that require little or no nitrogen input by making plants fix their own nitrogen, via the direct transfer of prokaryotic nitrogen fixation (nif) genes needed for the biogenesis of nitrogenase into the plant genome (Curatti and Rubio, 2014). This approach has the advantage of providing technology that can be transmitted to the progeny in the seeds. Significant proofs of concept have been achieved in this field, some of them derived from the work developed in this thesis (Allen et al., 2017; Burén et al., 2019; Burén, Young, et al., 2017; Eseverri et al., 2020; Ivleva et al., 2016; López-Torrejón et al., 2016). 


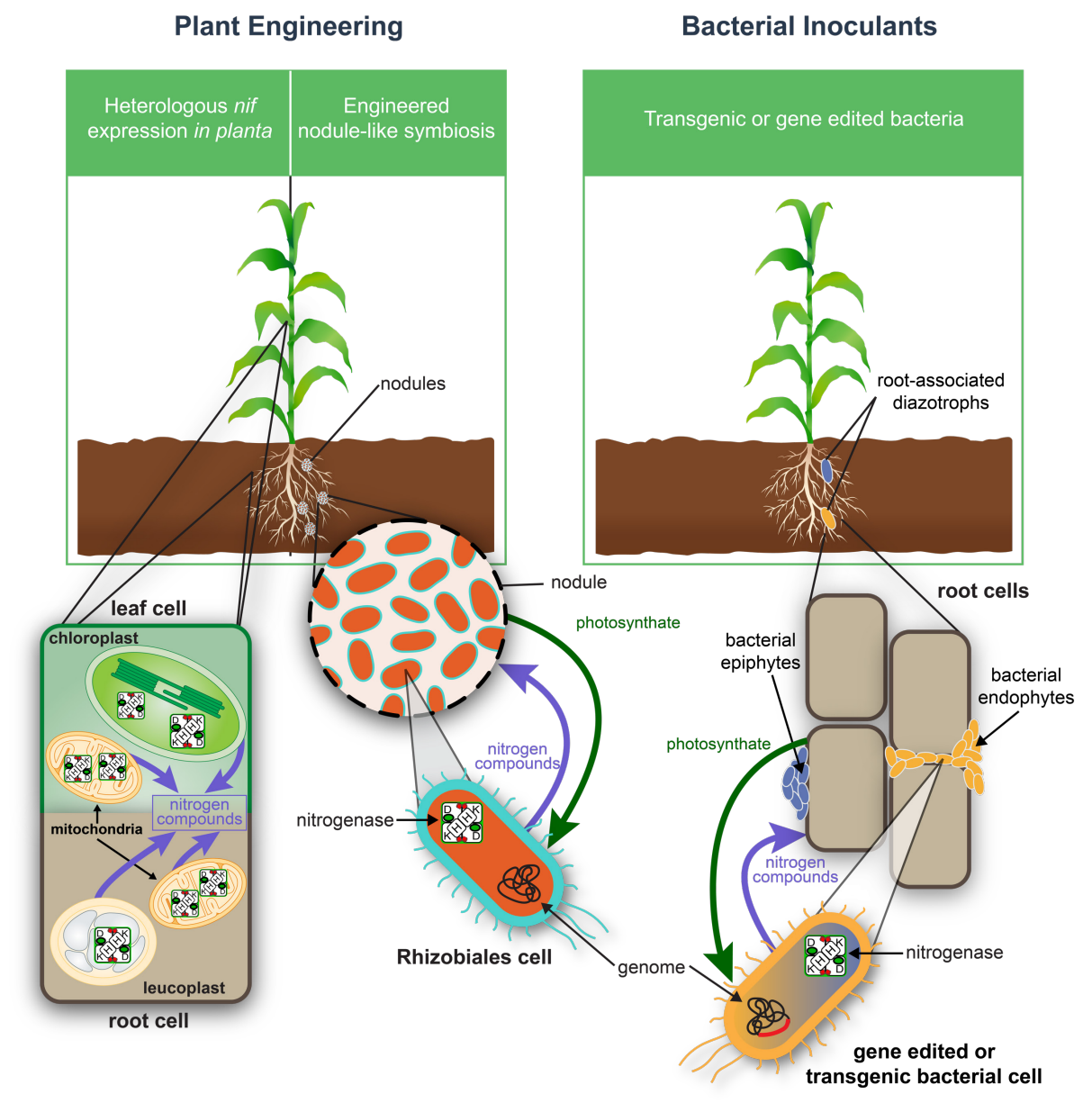

Figure 6. Biological nitrogen fixation in cereal crops as an alternative to industrial fertilizers.

Legumes form specialized symbioses with diazotrophic bacteria of the order Rhizobiales resulting in root nodule structures housing the rhizobia and providing them with sugars (photosynthate) in exchange for fixed nitrogen. Harnessing biological nitrogen fixation for cereal crops has been pursued via three different strategies: Enhancing nitrogen-fixing capabilities via genetic engineering of epiphytic or endophytic bacteria naturally associated with cereals and applying them to crops as inoculants; engineering new associations between cereals and nitrogen-fixing bacteria to recreate nodule-forming symbiosis; generating varieties of cereals that fix their own nitrogen via the direct transfer of prokaryotic nif genes into the plant genome and expression of nitrogenase in mitochondria and/or plastids. Adapted from Bloch et al. (2020).

\subsection{Nitrogenase biochemistry}

Nitrogenases can be classified into three groups according to their active site metal contents, i.e. vanadium (V) nitrogenase, iron only (Fe-only) nitrogenase and molybdenum (Mo) nitrogenase (Bishop and Joerger, 1990), being the last one the most abundant and ecologically relevant, therefore the best studied (Burén and Rubio, 2018).

Bacterial Mo-nitrogenase catalyses the reduction of dinitrogen into two molecules of ammonia in a reaction that requires eight electrons and the hydrolysis of at least 16 adenosine triphosphate molecules (ATP) (Seefeldt et al., 2009). The Mo-nitrogenase catalytic components consist of two metalloproteins: the nifH-encoded Fe protein 
homodimer and the nifDK-encoded MoFe protein heterotetramer (Figure 7) (Georgiadis et al., 1992). The Fe protein, also named dinitrogenase reductase, is a reductase that accepts electrons from ferredoxins or flavodoxins and functions as obligate electron donor to the MoFe protein. Besides, the Fe protein is required for the maturation of $\mathrm{P}$ clusters onto the MoFe protein and for the iron-molybdenum cofactor (FeMo-co) biosynthesis (Figure 8) (Burén et al., 2020). The Fe protein is a homodimer of the nifH gene product that contains one $\mathrm{Mg} \cdot \mathrm{ATP}$-binding site in each subunit and carries a single [4Fe-4S] cluster bridging the two subunits (Figure 8A) (Georgiadis et al., 1992). The MoFe protein, the catalytic component, is a dinitrogenase that binds and reduces molecular nitrogen. It is formed by a heterotetramer of the nifD and nifK gene products that carries one pair of complex metalloclusters in each NifDK half, the P-cluster and the FeMo-co (Figure 8B) (Kim and Rees, 1992).

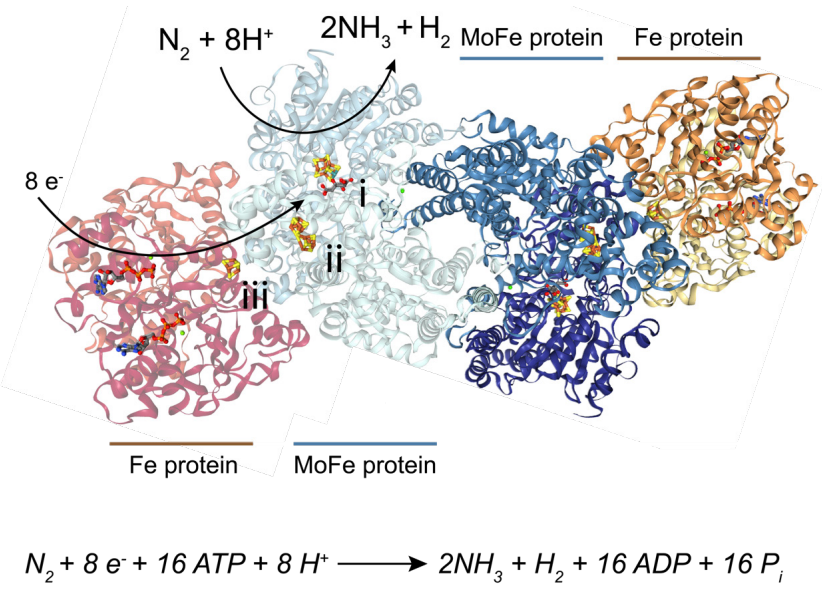

Figure 7. Structure and reaction of Mo nitrogenase.

The reduction of dinitrogen gas $\left(\mathrm{N}_{2}\right)$ to ammonia $\left(\mathrm{NH}_{3}\right)$ is catalysed by the nitrogenase enzyme complex, composed of two homodimers of the Fe protein, NifH, and one heterotetramer of the MoFe protein, NifDK. Structure of $A$. vinelandii MoFe protein and Fe protein complex showing (i) FeMo-co, (ii) Pcluster and (iii) $[4 \mathrm{Fe}-4 \mathrm{~S}]$ cluster in one half of the enzyme. Mo nitrogenase reaction is shown at the bottom. Adapted from Burén et al. (2020).

Remarkably, NifH is the most abundant Nif protein required for nitrogen fixation in $A$. vinelandii (Poza-Carrion et al., 2014). Its maturation consists of two steps: the correct folding and formation of the NifH homodimer, and the loading of the [4Fe-4S] cluster to generate a fully mature protein (Figure 8) (Burén et al., 2020). The presence of [4Fe-4S] cluster confers NifH its oxygen-sensitivity, as exposure to air irreversibly damages them and inactivates the enzyme (Shah and Brill, 1973).

Since the $\mathrm{Fe}$ protein component of nitrogenase is more oxygen-sensitive and its maturation requirements are simpler and better characterized, it has been chosen to test the possibility of engineering an active nitrogenase in yeast and plants (Burén et al., 2020; Shah and Brill, 1973). 
A.

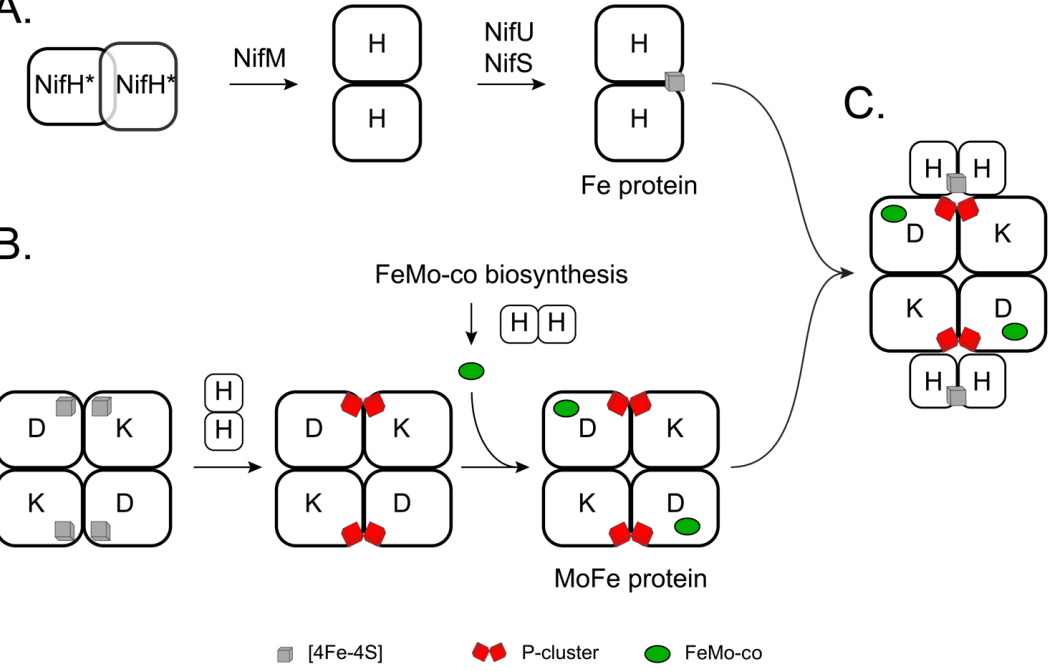

Figure 8. Simplified model of Fe protein maturation and its roles in MoFe protein maturation.

(A) Fe protein maturation: NifM assists in the correct folding of NifH, NifU and NifS assemble and transfer the $[4 \mathrm{Fe}-4 \mathrm{~S}]$ cluster to NifH. (B) Requirement of Fe protein for MoFe protein maturation: $\mathrm{Fe}$ protein is required for the maturation of P-clusters onto MoFe protein and for FeMo-co biosynthesis outside of the MoFe protein. Fe protein is shown as NifH homodimer lacking [4Fe-4S] cluster to indicate that apo-Fe protein is competent in P-cluster formation and FeMo-co biosynthesis. (C) Formation of mature Mo nitrogenase enzyme complex. Adapted from Burén et al. (2020).

The prokaryote model organism used as source of nitrogenase genes and proteins in this thesis is $A$. vinelandii, a free-living soil and strict aerobe bacterium that performs nitrogen fixation, in which nitrogenase is protected from oxygen damage mainly by maintaining high respiratory rates. There are four genes in A. vinelandii that are necessary to produce active Fe protein, namely nifH, nifM, nifS and nif $U$. The nif $M$ gene encodes a peptidylprolyl cis-trans isomerase that assists in the correct folding of NifH (Howard et al., 1986). The nifS gene product is a cysteine desulfurase that mobilizes $\mathrm{S}$ for $[\mathrm{Fe}-\mathrm{S}]$ cluster assembly that takes place on NifU (Zheng et al., 1994). Then, NifU, an assembly scaffold, sequesters $\mathrm{Fe}$ and $\mathrm{S}$ for $[\mathrm{Fe}-\mathrm{S}]$ groups and delivers them to nitrogenase target proteins for activity and cofactor synthesis (Fu et al., 1994).

The activity of the Fe protein can be easily determined by its capacity to support acetylene to ethylene formation by the MoFe protein in standard nitrogenase activity assays. In this biochemical assays, the heterologous Fe protein is incubated in vitro with a mixture of the remaining essential components, namely the MoFe protein purified from $A$. vinelandii, an electron donor (dithionite) and ATP (Shah and Brill, 1973). This assay relies on the ability of the triple-bonded molecule acetylene to serve as substrate for nitrogenase, in addition to dinitrogen, and its reduction to ethylene that can be detected by gas chromatography. 


\subsection{Engineering eukaryotic nitrogen fixation}

We face two main obstacles in the engineering of biological nitrogen fixation in plants. The first one is the complexity of the nitrogenase biosynthetic pathway, which requires the co-expression of eight to ten bacterial genes in a precise stoichiometry to achieve an enzyme with optimal function (Smanski et al., 2014; Temme et al., 2012; Vicente and Dean, 2017; Wang et al., 2013). The second hurdle is the sensitivity of the nitrogenase enzyme and the accessory proteins involved in the synthesis of its metalloclusters towards oxygen, a by-product of photosynthesis in plants (Curatti and Rubio, 2014). In addition, for nitrogenase to function properly it requires abundant available reducing power and ATP.

The DBTL cycle has been used to overcome the complexity barrier, testing each one of the nitrogenase components and validating their functionality. For this purpose, the model eukaryote Saccharomyces cerevisiae and plant $N$. benthamiana have been used as chassis to gain relevant information with the idea of transferring it to cereal engineering.

To solve the oxygen sensitivity problem, the subcellular organelles mitochondria and chloroplast (plastid) have been proposed as possible compartments in which to engineer nitrogenase in plants (Beatty and Good, 2011; Curatti and Rubio, 2014). The mitochondrial matrix provides several advantages. It presents a low oxygen environment due to respiration, it can provide the ATP and reducing power required for nitrogenase catalytic activity, and carries iron-sulfer ([Fe-S]) cluster assembly machineries (Lill and Mühlenhoff, 2008). However, a recent study showed that mitochondrial ferredoxins cannot directly replace the function of their counterparts in bacteria by serving as electron donors to nitrogenase, increasing the complexity of engineering an active mitochondrial nitrogenase (Yang et al., 2017). Alternatively, chloroplasts are considered as a potential subcellular location because of their availability of the immediate products of photosynthesis, NADPH and ATP, that could satisfy the demands of reducing power and energy, as well as, the presence of [Fe-S] cluster assembly machineries needed for nitrogenase biosynthesis (Balk and Pilon, 2011; Curatti and Rubio, 2014). Moreover, the same study from Yang and colleagues demonstrated that a number of ferredoxins found in plastids are able to serve as electron donors to nitrogenase (Yang et al., 2017). However, an obvious disadvantage is the oxygen production as a by-product of photosynthesis that takes place in chloroplasts, which may be incompatible with the oxygen-labile nitrogenase (Curatti and Rubio, 2014).

All considered, chloroplasts may have the necessary properties to accommodate the assembly of a functional nitrogenase enzyme, but to overcome the oxygen sensitivity 
problem, its biosynthesis might need to be temporally separated from photosynthesis, as it is the case in some photosynthetic nitrogen-fixing cyanobacteria known to accommodate photosynthesis and nitrogen fixation separating them temporally under the control of the circadian clocks (Grobbelaar et al., 1986; Mitsui et al., 1986).

\subsection{Coupling nitrogen fixation to plant metabolism}

The subcellular locations proposed for the engineering of nitrogenase in plants need to consider the appropriate physiological environment for the enzyme, as well as the metabolic coupling to the plant host. Poor metabolic coupling of recombinant pathways has shown to directly affect performance in hosts (Ortiz-Marquez et al., 2013) and it could lead to side effects influencing the host system itself (Wilson and Roberts, 2014). Even if the expression of a nitrogenase enzyme at sufficient levels in plant mitochondria or plastids is achieved, several factors may limit efficient nitrogen fixation.

The first constraint relates to the provision of sufficient reductant through the Fe protein as an obligated donor of electrons to the MoFe protein. The fact that the in vivo electron donor for the Fe protein is less strictly conserved, opens the possibility to find compatible plant counterparts which would allow reducing the number of genes required for nitrogenase engineering (Halbleib and Ludden, 2000; Yang et al., 2017).

The second factor faces the biosynthesis of metalloclusters in plant organelles. In plants, [Fe-S] cluster assembly machineries capable of synthesizing [4Fe-4S] clusters are found in mitochondria (ISC pathway) and chloroplasts (SUF pathway) (Balk and Pilon, 2011). These machineries from eukaryotic organelles can substitute to an extent the activity of NifU and NifS in harbouring and incorporating the [4Fe-4S] clusters into NifH (Ivleva et al., 2016; López-Torrejón et al., 2016). However, the complex cofactors carried by the MoFe protein, FeMo-co and P-cluster, are only found in nitrogen fixers.

A third constraint is how to couple the ammonia production as a result of nitrogen fixation into the host pathways, as it can be toxic to plants. In plants, ammonia assimilation takes place primarily in chloroplasts and other plastids by the glutamine synthetase/glutamate synthase pathway, where it is incorporated into the formation of amino acids (Foyer et al., 2011; Merrick and Dixon, 1984). Thus, it seems possible to easily couple the ammonia produced by nitrogenase into host metabolism in plastids. In the case of mitochondria, ammonia produced could be exported to the cytosol and assimilated by a cytosolic glutamine synthetase using glutamate from the ornithine catabolism or loaded into the xylem (Good, 2018; Witte, 2011). 
The fourth factor is the energetic requirement, as nitrogenase uses large amounts of ATP. Both mitochondria and chloroplast can theoretically provide enough ATP for nitrogenase to function but the metabolic burden that it may carry is still unknown. Nevertheless, the theoretical energy requirement for nitrogen fixation is almost identical to that required for nitrate assimilation, the other main source of nitrogen for most field crops (Kennedy and Tchan, 1992).

\subsection{State of the art in the engineering of nitrogen-fixing organisms}

We encounter ourselves far from engineering a plant that is able to fix nitrogen. Nevertheless, significant advances have been achieved in the last years in photosynthetic prokaryotes and in eukaryotic organisms following different approaches.

\subsubsection{Engineering nitrogen fixation in a non-diazotrophic cyanobacterium}

Engineering nitrogen fixation in a non-diazotrophic cyanobacterium may prove insightful, as cyanobacteria are considered the ancestors of chloroplasts (Falcón et al., 2010; Jarvis and López-Juez, 2013). Fifteen years ago, Cheng et al. (2005) proved that the nifH gene from Klebsiella pneumoniae M5a1 (now renamed as K. oxytoca) could partially restore the capacity for chlorophyll biosynthesis in the dark in Chlamydomonas reinhardtii. Recently, Tsujimoto et al. (2018) demonstrated the low, but functional, expression of an oxygen labile nitrogenase from the diazotrophic cyanobacterium Leptolyngbya boryana in an oxygenic photosynthetic organism, the non-diazotrophic cyanobacterium Synechocystis sp. PCC 6803. Furthermore, Liu et al. (2018) successfully engineered the same organism to express a nif gene cluster from Cyanothece sp. ATCC 51142 , achieving nitrogenase activity up to $30 \%$ of the wild-type enzyme. Interestingly, the introduction of uptake hydrogenase genes showed to improve nitrogenase activity under micro-oxic conditions.

\subsubsection{Engineering nitrogen fixation in yeast}

S. cerevisiae is a widely used eukaryotic chassis for synthetic biology, serving as a starting point for complex engineering projects in higher eukaryotes. The formation of a functional nitrogenase enzyme has not yet been achieved in yeast mitochondria, although all the main Nif components have been individually expressed in it (Burén, Jiang, et al., 2017; Burén, Young, et al., 2017; López-Torrejón et al., 2016; Pérez-González et al., 2017). The first breakthrough came from the study by López-Torrejón et al. (2016), where active Fe protein was produced from $A$. vinelandii nifH and nif $M$ genes co-expression in the mitochondrial matrix of yeast cultures growing under highly aerobic conditions. This study confirmed the protective function against oxygen offered by respiration in the 
mitochondria and that the endogenous mitochondrial $[\mathrm{Fe}-\mathrm{S}]$ cluster assembly machinery can substitute, at least to an extent, some of the functions of NifS and NifU. Burén, Young et al. (2017) demonstrated that expression and mitochondrial targeting of a nine-gene nif cluster of $A$. vinelandii in yeast resulted in NifDK tetramer formation, although the resulting MoFe protein was inactive. The second milestone was achieved by Burén et al. (2019) with the production of active NifB and its product NifB-co in yeast mitochondria, which are key to the biosynthesis of the Mo-nitrogenase active-site cofactor, i.e. FeMoco, though in this case co-expression with NifU and NifS was required.

\subsubsection{Engineering nitrogen fixation in plants}

Engineering a staple crop capable of fixing its own nitrogen is the ultimate goal of the 'direct gene transfer' strategy. To date, the expression of Mo-nitrogenase components in both mitochondria and chloroplast of model plants, namely $N$. benthamiana or Nicotiana tabacum, has been achieved.

\subsubsection{Mitochondria}

Studies targeting mitochondria as a subcellular location for the expression of nitrogenase components have appeared in recent years. Allen et al. (2017) showed individual transient expression of 16 Nif proteins from $K$. oxytoca targeted to $N$. benthamiana mitochondria, but none of the structural components was tested for activity. In the case of NifD, a mitochondrial endoprotease was degrading the protein once imported into the mitochondria due to a specific motif found in its amino acid sequence. Its mutation generated NifD variants resistant to degradation in both plant and yeast (Allen et al., 2020; Xiang et al., 2020).

An exploration to identify superior Mo-nitrogenase components from other organisms, that could outperform those of $K$. oxytoca and $A$. vinelandii has been underway (Burén et al., 2019). In this context, Jiang et al. (2020) just reported that the Fe protein from Hydrogenobacter thermophilus was found active when targeted to N. benthamiana mitochondria.

\subsubsection{Chloroplast}

More than twenty years ago, Dixon et al. (1997) showed that nifH and nifM genes from $K$. pneumoniae N-terminally fused to a transit peptide were correctly expressed and imported into chloroplast of $N$. tabacum plants. Unfortunately, the NifH protein levels achieved were very low and no activity could be determined. In an alternative approach, Ivleva et al. (2016) generated transplastomic tobacco plants expressing the nifH and nifM 
genes of $A$. vinelandii and showed low Fe protein activity from plant enriched extracts, although only when plants were prior cultivated in a low-oxygen atmosphere. Given these scattered results after 25 years of work, it seemed plausible that the lack of positive reports could be due to the presence of oxygen-derived from the photosynthetic activity of plant chloroplasts, which could be avoiding the accumulation of functional nitrogenase proteins in this subcellular location.

\subsection{Engineering of nitrogenase biosynthesis in plastids}

SynBio projects in chloroplasts of higher plants have proven a useful strategy for the last two decades (Boehm and Bock, 2019). To definitely test the suitability of plastids as locations for the engineering of nitrogenase biosynthesis, we envisioned two technical possibilities.

\subsubsection{Plastid transformation}

The first one consists of the direct transformation of the chloroplast genome by stacking prokaryotic nitrogenase transgenes in synthetic operons, generating transplastomic plants. This approach presents important advantages, such as avoidance of gene silencing and epigenetic or positional effects, elevated protein expression levels due to high plastid DNA copy number and transgene containment due to plastid maternal inheritance in most crops (Adem et al., 2017). However, chloroplast transformation methods are not yet in place for staple crop plants or are in the best case problematic, due to a naturally present point mutation that confers spectinomycin resistance in the plastid 16S rRNA genes of all cereals (Bock, 2015). Although chloroplast genomes of several major crops including cotton, soybean or sugar beet have been transformed, cereals such as maize and rice remain elusive to this technique (Clarke and Daniell, 2011). As our research aims to generate nitrogen-fixing cereals, this technical impossibility pushed our efforts towards the second possible strategy.

\subsubsection{Nuclear transformation}

The second strategy is based on nuclear genome transformation, following a monocistronic gene structure, and targeting of the recombinant cytosol-translated proteins into the chloroplast by fusing them to chloroplasts transit peptides.

The major advantage of nuclear transformation lies in the existence of techniques to transform most of the known plant species amenable to engineering. Some bottlenecks, like the assembly of large DNA constructs, enough to encode for all proteins required for nitrogenase biosynthesis, has been facilitated by the development of modular assembly 
techniques in the last decade and there is a growing collection of characterized genetic modules, e.g. promoters, terminators, gene switches or insulators, that enables a tightly controlled regulation of transgene expression when integrated within the host genome (Bernabé-Orts et al., 2020; Cai et al., 2020; Engler et al., 2014; Patron, 2020; PérezGonzález et al., 2018; Vazquez-Vilar et al., 2016, 2017). Moreover, a relevant asset of nuclear transformation is the quick estimate of the performance of constructions that can be made for gene expression and protein targeting with transient expression assays.

For this approach to work, it is critical that protein import from transit peptides is highly efficient. The use of chloroplast transit peptides is a strategy that has already been used in many biotechnological applications to direct recombinant proteins to the chloroplast stroma (Carvalho et al., 2011; Feng et al., 2010; Kebeish et al., 2007; Kiani et al., 2013; Maier et al., 2012; Shen et al., 2019, 2017). However, targeting foreign proteins to chloroplasts can still be a troublesome task in some cases (Shen et al., 2017) and there is a need for the characterization of specific signals that perform correctly in the species of choice.

The collection of transit peptides available at the moment in which we started this project, although functional, was not optimal. Most of the TPs described in the literature included a sequence longer than the one cleaved after import, leaving undesired amino acid residues in the $\mathrm{N}$-terminus of the recombinant proteins, which can affect folding and function (Muthamilselvan et al., 2019). Furthermore, although targeting of proteins to mitochondria and chloroplasts is generally highly specific, an increasing number of proteins imported dually into both organelles have been described (Peeters and Small, 2001).

The use of SynBio tools for the engineering of complex pathways in higher plant chloroplasts has proved its potential during the last years. In this thesis, we targeted chloroplasts from $N$. benthamiana and $O$. sativa and generated the proof of concept that nitrogenase biosynthesis can be engineered in plant plastids. 



\section{Material and Methods}

\section{DNA Techniques}

\subsection{Modular Cloning Techniques}

All constructs used in this work were generated using MoClo (Werner et al., 2012) and its adaptation for the direct DNA transfer to rice (Baysal et al., 2020).

\subsubsection{Standard MoClo conditions}

MoClo restriction-ligations were set up in a final volume of $20 \mu \mathrm{l}$ with a 2:1 molar ratio of insert:acceptor vector, $5 \mathrm{U}$ of the required restriction enzyme - BpiI (Thermo Fisher Scientific) for the generation of Level 0 and Level 2 plasmids, and BsaI-HFv2 (NEB) for the generation of Level 1 plasmids - 4.5U of T4 Ligase (Promega), $1.5 \mu$ l of Ligase 10x Buffer and $1.5 \mu \mathrm{l}$ of 10x BSA (GE Healthcare). The reactions were incubated in a thermocycler with the following program: $20 \mathrm{~s}$ at $37^{\circ} \mathrm{C}, 26$ cycles of $3 \mathrm{~min}$ at $37^{\circ} \mathrm{C}$ plus $4 \mathrm{~min}$ at $16^{\circ} \mathrm{C}$, and $5 \mathrm{~min}$ at $50^{\circ} \mathrm{C}, 5 \mathrm{~min}$ at $80^{\circ} \mathrm{C}$ and hold at $16^{\circ} \mathrm{C}$.

The reaction mixes were then added to chemically competent cells of E. coli DH5 $\alpha$ or TOP10 (Thermo Fisher Scientific), which were then incubated in LB solid medium containing $20 \mu \mathrm{g} / \mathrm{ml} \mathrm{X-Gal} \mathrm{(Duchefa} \mathrm{Biochemie),} 1 \mathrm{mM}$ IPTG (Sigma-Aldrich), and the corresponding antibiotic, $50 \mu \mathrm{g} / \mathrm{ml}$ spectinomycin (Sigma-Aldrich) for Level 0 or Level M, $100 \mu \mathrm{g} / \mathrm{ml}$ carbenicillin (Formedium) for Level 1, or $50 \mu \mathrm{g} / \mathrm{ml}$ kanamycin (Formedium) for Level 2 constructs. Colonies were selected for plasmid DNA (pDNA) extraction using the QIAprep Spin Miniprep Kit (Qiagen). After pDNA extraction, concentrations were determined using NanoDrop ${ }^{\circledR}$ ND-1000 (Thermo Fisher Scientific). The fidelity of all plasmids was verified by Sanger sequencing (Eurofins Genomics) and results were analysed using Geneious version 10.2.2 software (Kearse et al., 2012).

E. coli colonies corresponding to positive clones were stored in $60 \%$ glycerol stocks in cryovials (Nalgene) at $-80^{\circ} \mathrm{C}$.

\subsubsection{Generation of modular pieces}

The design of primers for amplification of DNA fragments to generate Level 0 plasmids was performed manually following the rules described in Weber et al. (2011). When DNA fragments contained internal restriction sites for either BsaI or BpiI, the fragment was domesticated using internal primers to eliminate the site. The design of domestication primers was performed using the Domesticator Tool 
(https://gbcloning.upv.es/do/domestication/), subsequently modifying the tails of the primers to add a BpiI restriction site.

Modular DNA fragments were amplified by PCR using Phusion Hot Start II DNA Polymerase (Thermo Fisher Scientific), following manufacturer's protocol, and the primers and templates described in Supplemental Table 1. PCR products were analysed in 1\% agarose gels to check for correct amplicon size, exclude possible non-specific amplicons and primer dimers, and gel purified using QIAquick Gel Extraction Kit (Qiagen).

All modular pieces generated in this thesis used the Universal Level 0 vector (pAGM9101) from the MoClo Toolkit (Werner et al., 2012) as an entry vector. Modular pieces cloned for this work can be found in Supplemental Table 2.

\subsubsection{Generation of Transcriptional Units}

In the MoClo system, pieces are assembled into TUs in restriction/ligation reactions with destination vectors that define the position of each TU in a future multigene assembly.

Level 1 in all six different positions $\left(\mathrm{L}_{\mathrm{P}} \mathrm{P}_{\mathrm{x}}\right)$ were used as destination vectors, either binary vectors for Agrobacterium T-DNA delivery to plant cells (pICH47732-L1P1, pICH47742-L1P2， pICH47751-L1P3， pICH47761-L1P4， pICH47772-L1P5， and pICH47781-L1P6 ) (Werner et al., 2012) or vectors adapted for particle bombardment transformation of plants (pUC57-L1P1, pUC57-L1P2, pUC57-L1P3, pUC57-L1P4, pUC57-L1P5, and pUC57-L1P6) (Baysal et al., 2020 and Section 3.1.5).

A list of all Level 1 constructs generated and used in this work can be found in Supplemental Table 3.

\subsubsection{Generation of multigene constructs}

Multigene constructs were assembled from Level 1 constructs in acceptor backbones named Level 2 or M (Werner et al., 2012). For this work, Level 2 binary vectors for Agrobacterium T-DNA delivery to plant cells (pAGM4673-L2 and pAGM8055-LM) (Werner et al., 2012) or a Level 2 vector adapted for particle bombardment transformation of plants (pUC57-L2) (Baysal et al., 2020) were used as destination vectors.

A list of all Level 2 and $\mathrm{M}$ constructs generated and used in this work can be found in Supplemental Table 4. 


\subsubsection{Adaptation of MoClo vectors for particle bombardment}

The backbone of the pUC57-AmpR (GenScript) vector was PCR amplified using Phusion Hot Start II DNA Polymerase (Thermo Fisher Scientific) and primers 591/592, following the manufacturer's protocol. The PCR product was analysed in a 1\% agarose gel and a band corresponding to over 2200 bp was purified using QIAquick Gel Extraction Kit (Qiagen) and digested with AdeI (Thermo Fisher Scientific) following the manufacturer's protocol.

The vectors pICH47732-L1P1, pICH47751-L1P3, pICH47761-L1P4, pICH47772-L1P5, and pICH47781-L1P6 (Werner et al., 2012) were digested with AdeI, analysed in 1\% agarose gels, and fragments of $650 \mathrm{bp}$ corresponding to the MoClo cloning cassettes were purified.

The AdeI-digested cloning cassettes were ligated to the amplified pUC57-AmpR backbone using T4 Ligase (Promega), according to the manufacturer's indications. Ligation mixtures were transformed in E. coli DH5 $\alpha$ chemically competent cells, which were grown on LB solid medium containing $20 \mu \mathrm{g} / \mathrm{mL}$ X-Gal (Duchefa) and 1mM IPTG (Sigma-Aldrich), supplemented with $100 \mu \mathrm{g} / \mathrm{mL}$ carbenicillin (Formedium).

Blue carbenicillin resistant colonies were selected for plasmid DNA extraction using the GenElute Plasmid Miniprep Kit (Sigma-Aldrich). The ligation between backbone and cassette was verified by Sanger sequencing (Eurofins Genomics) and results were analysed using Geneious version 10.2.2 software (Kearse et al., 2012).

Vector can be found in Addgene under the ID 109220 (pUC57-L1P1), 109222 (pUC57L1P3), 109223 (pUC57-L1P4), 109224 (pUC57-L1P5), and 109225 (pUC57-L1P6).

\subsection{Multiple Overlap Extension PCR}

Point mutations in rice codon-optimized genes nifH, nifM, nif $U$, and nifS were performed via multiple overlap extension PCR (MOE-PCR) as described in (Kadkhodaei et al., 2016) with slight modifications.

Firstly, genes were PCR amplified in several fragments with primers 887 to 910, introducing the silent mutations described in Supplemental Tables 1 and 2, using Phusion Hot Start II DNA Polymerase (Thermo Fisher Scientific), setting up reactions according to the manufacturer's protocol. Each fragment had an overlapping section of about $20 \mathrm{bp}$ with the contiguous section, in order to set a correct order in the MOE-PCR step. 
PCR products were analysed in 1\% agarose gels to check for correct amplicon size and exclude possible non-specific amplicons and primer dimers, and gel purified using QIAquick Gel Extraction Kit (Qiagen).

In order to build the full nif genes, $100 \mathrm{ng}$ of each linear fragment was mixed in a $50 \mu \mathrm{L}$ MOE-PCR reaction containing all reagents and primers (613 to 618,623, and 624) for the amplification of the modular piece. Thermal cycling conditions were as follows: $30 \mathrm{~s}$ at $95^{\circ} \mathrm{C},\left[10 \mathrm{~s}\right.$ at $98^{\circ} \mathrm{C}, 80-70^{\circ} \mathrm{C}$ (gradient as $0.4^{\circ} \mathrm{C} / \mathrm{s}$ ), $30 \mathrm{~s}$ at $\left.72^{\circ} \mathrm{C}\right]$ for 30 cycles and a final extension of $5 \mathrm{~min}$ at $72^{\circ} \mathrm{C}$.

After running the MOE-PCR reaction, the products were analysed in 1\% agarose gels and gel purified using the QIAquick Gel Extraction Kit (Qiagen). Linear DNA was used in MoClo restriction/ligations reactions to generate modular pieces as described in Section 3.1.2.

\subsection{Genomic DNA isolation}

Genomic DNA isolation of A. thaliana Col-0, N. tabacum L. cv. Samsun, O. sativa subsp. japonica and Pisum sativum were necessary as a template for PCR amplification to clone modular pieces (see Supplemental Table 2). Isolation was performed using the DNeasy Plant Mini Kit (Qiagen) except for P. sativum, which was performed using Extract-NAmp ${ }^{\text {TM }}$ Plant Tissue PCR Kits (Sigma-Aldrich) following the manufacturer's protocol.

\section{Bacterial Culture Techniques}

\subsection{Escherichia coli}

DH5 $\alpha$ strain ( $\mathrm{F}^{-}$Ф80lacZ $\Delta \mathrm{M} 15 \Delta$ (lacZYA-argF) U169 recA1 endA1 hsdR17( $\left.\mathrm{r}_{\mathrm{k}}{ }^{-}, \mathrm{m}_{\mathrm{k}}{ }^{+}\right)$ phoA supE44 thi-1 gyrA96 relA1 $\lambda^{-}$) (Thermo Fisher Scientific) and TOP10 strain (F$m c r A \Delta(m r r-h s d \mathrm{RMS}-m c r \mathrm{BC})$ 980lacZ $\Delta \mathrm{M} 15 \Delta$ lacX74 recA1 araD139 $\Delta$ (araleu)7697 galU galK rpsL (StrR) end $\mathrm{A} 1$ nup $\mathrm{G}$ ) (Thermo Fisher Scientific) were used for cloning purposes.

E. coli cells were grown at $37^{\circ} \mathrm{C}$ in $\mathrm{LB}$ solid or liquid medium (Bertani, 1951), supplemented with antibiotics, X-Gal (Duchefa) or IPTG (Sigma-Aldrich) as needed.

For plasmid transformation, chemically competent E. coli cells were prepared as described in Hanahan et al. (1991). 


\subsection{Agrobacterium tumefaciens}

A. tumefaciens GV3101 (pMP90) strain (Koncz and Schell, 1986) cells were grown at $28-30^{\circ} \mathrm{C}$ in $\mathrm{LB}$ medium containing rifampicin $(25 \mu \mathrm{g} / \mathrm{mL})$ (Sigma-Aldrich), and gentamicin $(10 \mu \mathrm{g} / \mathrm{mL})$ (Sigma-Aldrich), supplemented with other selective antibiotics as needed. For liquid cultures, cells were grown in baffled flasks. For plasmid transformation, chemically competent $A$. tumefaciens cells were prepared following the same protocol as for E. coli described in Hanahan et al. (1991).

\section{Plant Culture Techniques}

\subsection{Nicotiana benthamiana}

\subsubsection{Growth conditions}

$N$. benthamiana plants were cultivated on $8 \times 8 \times 8 \mathrm{~cm}$ pots in a greenhouse in long-day conditions (16 h light/8 h dark) ensuring enough light with LED lamps at $26 / 22^{\circ} \mathrm{C}$ day/night temperature.

\subsubsection{Transient expression assays}

Overnight cultures of A. tumefaciens GV3101 strain cells carrying the plasmid of interest were diluted to an $\mathrm{OD}_{600}$ between 0.3-0.6 in an infiltration solution containing $10 \mathrm{mM}$ MES pH 5.5, $10 \mathrm{mM} \mathrm{MgSO}_{4}$, and $150 \mu \mathrm{M}$ acetosyringone (Sigma-Aldrich) and incubated at $150 \mathrm{rpm}$ at room temperature (RT) for $3 \mathrm{~h}$. When using single transcriptional units, an A. tumefaciens GV3101 strain carrying the silencing suppressor p19 (Li, 2011; Naim et al., 2012) was co-diluted to an $\mathrm{OD}_{600}$ of 0.3-0.6 (multigenic constructs already contain p19 transcriptional unit). Leaves of 3-4 weeks-old $N$. benthamiana plants were agroinfiltrated using a syringe without a needle and 3-4 days after infiltration, tissue was collected at the end of the dark period and used for protein extraction, chloroplast isolation, or confocal microscopy (protocols performed under ambient light).

\subsection{Arabidopsis thaliana}

\subsubsection{Seed sterilization}

Seeds were sterilized in $1.5 \mathrm{~mL}$ tubes using a solution of $20 \%(\mathrm{v} / \mathrm{v})$ commercial bleach and $0.1 \%(\mathrm{v} / \mathrm{v})$ Tween 20 (VWR Chemicals). Seeds were incubated in that solution for 10 min under constant agitation, followed by five washes with sterile MilliQ water.

After sterilization, tubes were covered with tinfoil and seeds remained in the dark for 23 days at $4^{\circ} \mathrm{C}$ for stratification. 


\subsubsection{Culture media}

A. thaliana Col-0 seeds were plated in MS medium pH 5.7 (Murashige and Skoog, 1962) (Mod.No.1B, Duchefa) with 1\% (w/v) sucrose (Duchefa) and 1\% (w/v) plant agar (Duchefa).

\subsubsection{Growth conditions}

Seedlings were grown in an environmentally controlled chamber under 16/8 h light/dark conditions at $22^{\circ} \mathrm{C}$ and $65 \%$ relative humidity $(\mathrm{RH})$.

\subsubsection{Protoplast isolation}

Isolation of protoplast from Arabidopsis seedlings was performed as described in Yoo et al. (2007) and González-García et al. (2020) with modifications. Aerial parts and roots of ca. 100 seedlings were separated and cut into $0.5-1.0 \mathrm{~mm}$ strips using a razor blade. The strips were transferred to different Erlenmeyer flasks containing $10 \mathrm{ml}$ of enzyme solution (1.25\% (w/v) cellulase R10 (Duchefa), 0.3\% (w/v) macerozyme R10 (Duchefa), $0.4 \mathrm{M}$ D-mannitol, $20 \mathrm{mM}$ MES at $\mathrm{pH}$ 5.7, $20 \mathrm{mM} \mathrm{KCl,} 10 \mathrm{mM} \mathrm{CaCl}_{2}$ and $0.1 \%$ (w/v) BSA) and incubated in the dark for $3 \mathrm{~h}$ (aerial part) or $1 \mathrm{~h}$ and 45 min (roots) at RT with $80 \mathrm{rpm}$ shaking to allow digestion of cell wall material. An equal amount of W5 solution (154 $\mathrm{mM} \mathrm{NaCl}, 125 \mathrm{mM} \mathrm{CaCl}_{2}, 5 \mathrm{mM} \mathrm{KCl}$ and $2 \mathrm{mM}$ MES at $\mathrm{pH}$ 5.7) was added to stop the digestion. Protoplasts were released by filtering through $40 \mu \mathrm{m}$ nylon cell strainers and pelleted by centrifugation at $200 \times g$ for 2 min (aerial part) or $500 \times g$ for $10 \mathrm{~min}$ (roots) at RT. Protoplasts were resuspended in $2 \mathrm{ml} \mathrm{MMG} \mathrm{solution} \mathrm{(0.4} \mathrm{M} \mathrm{D-mannitol,}$ $15 \mathrm{mM} \mathrm{MgCl}$, and $4 \mathrm{mM}$ MES at $\mathrm{pH}$ 5.7) and kept on ice.

\subsubsection{Protoplast transfection}

Transformation of protoplasts was carried out by DNA-PEG-calcium transfection as described in Yoo et al. (2007) and Page et al. (2019) with modifications. For each transformation, $10 \mu \mathrm{l}$ of plasmid (5 to $10 \mu \mathrm{g}$ DNA) and $100 \mu \mathrm{l}$ of protoplasts were combined with $110 \mu \mathrm{l}$ of a freshly-prepared PEG solution containing 40\% (w/v) PEG 4000 (Merk), 0.2 M D-mannitol and $0.1 \mathrm{M} \mathrm{CaCl}_{2}$, and incubated in the dark at RT for 15 min. Negative control samples, replacing plasmid with $10 \mu \mathrm{l}$ Milli-Q $\mathrm{H}_{2} \mathrm{O}$, were carried out. W5 solution ( $440 \mu \mathrm{l})$ was slowly added to complete transformations. Protoplasts were pelleted by centrifugation at $100 \times g$ for $2 \mathrm{~min}$ in the case of leaf protoplasts or $500 \times g$ for $10 \mathrm{~min}$ for root protoplast, resuspended in $375 \mu \mathrm{l}$ of WI solution (0.5 M D-mannitol, $20 \mathrm{mM} \mathrm{KCl}$ and $4 \mathrm{mM}$ MES at $\mathrm{pH}$ 5.7), transferred to 96-well microplates (125 $\mu 1$ per well), and incubated at RT on the laboratory bench for $16 \mathrm{~h}$. 


\subsection{Oryza sativa}

\subsubsection{Transient expression assays}

\subsubsection{Growth of plant material}

Rice seeds (Oryza sativa L. cv. EYI 105) were sown into a 3:1 mixture of soil (Floragard) and vermiculite in $\varnothing 13 \mathrm{~cm}$ pots and grown for nine days after germination in an environmentally controlled chamber (Conviron) under 16/8 h light/dark conditions at $24^{\circ} \mathrm{C}$ and $70 \% \mathrm{RH}$.

\subsubsection{Protoplast isolation}

Isolation of protoplast from rice seedlings was performed as described in Zhang et al. (2011) and Page et al. (2019) with slight modifications. About 100 seedlings were harvested and approximately $10 \mathrm{~cm}$ of stem and sheath green tissue was retained and subsequently cut into $0.5-1 \mathrm{~mm}$ strips using a razor blade. The strips were immediately submerged into 0.6 M D-mannitol (Acros Organics) for $15 \mathrm{~min}$ in the dark at RT to initiate plasmolysis.

After discarding the mannitol, the tissue was transferred to an enzyme solution $(1.5 \%$ (w/v) cellulase RS (Duchefa), 0.75\% (w/v) macerozyme R10 (Duchefa), 0.6 M Dmannitol (Acros Organics), $20 \mathrm{mM}$ MES at pH 5.7 (Duchefa), $10 \mathrm{mM} \mathrm{KCl} \mathrm{(Acros}$ Organics), $10 \mathrm{mM} \mathrm{CaCl} 2$ (Merk) and 0.1\% (w/v) BSA (GE Healthcare Life Sciences)) and incubated in the dark for $4 \mathrm{hr}$ at RT with $60 \mathrm{rpm}$ shaking to allow digestion of cell wall material. An equal amount of W5 solution (154 mM NaCl, $125 \mathrm{mM} \mathrm{CaCl}_{2}, 5 \mathrm{mM}$ $\mathrm{KCl}$ and $2 \mathrm{mM}$ MES at $\mathrm{pH} 5.7$ ) was added to terminate digestion.

Protoplasts were released by filtering through $40 \mu \mathrm{m}$ nylon cell strainers (Corning) with 3 washes of the strips using W5 solution. The pellets were collected by centrifugation at $300 \times g$ for $3 \mathrm{~min}$ at RT. The supernatant was decanted, and the pellets were washed by gentle resuspension in $10 \mathrm{ml} \mathrm{W5}$ solution. After second centrifugation at $300 \times g$ for 3 min at RT, the pellets were resuspended in 1 to $2 \mathrm{ml} \mathrm{MMG}$ solution (0.4 M D-mannitol, $15 \mathrm{mM} \mathrm{MgCl}_{2}$, and $4 \mathrm{mM}$ MES at $\mathrm{pH}$ 5.7).

\subsubsection{Protoplast transfection}

Transformation of protoplasts was carried out by DNA-PEG-calcium transfection as described in (Page et al., 2019). For each transformation, $10 \mu \mathrm{l}$ of plasmid (5 to $10 \mu \mathrm{g}$ DNA) and $120 \mu$ of protoplasts were combined with $140 \mu$ of a freshly-prepared PEG solution containing 40\% (w/v) PEG 4000 (Merk), $0.2 \mathrm{M}$ D-mannitol and $0.1 \mathrm{M} \mathrm{CaCl}_{2}$, and incubated in the dark at RT for $25 \mathrm{~min}$. Negative control samples, replacing plasmid 
with $10 \mu \mathrm{l}$ Milli-Q $\mathrm{H}_{2} \mathrm{O}$, were carried out. W5 solution $(560 \mu \mathrm{l})$ was slowly added to complete transformations. Protoplasts were pelleted by centrifugation, resuspended in $375 \mu \mathrm{l}$ of WI solution ( $0.5 \mathrm{M} \mathrm{D}$-mannitol, $20 \mathrm{mM} \mathrm{KCl}$ and $4 \mathrm{mM} \mathrm{MES}$ at $\mathrm{pH}$ 5.7), transferred to 96 -well microplates $(125 \mu \mathrm{l}$ per well), and incubated at RT on the laboratory bench for 12 to $16 \mathrm{~h}$.

\subsubsection{Stable transformation}

\subsubsection{Seed sterilization}

Rice seeds (Oryza sativa L. cv. EYI 105) were dehusked, surface-sterilized in 70\% (v/v) ethanol for $3 \mathrm{~min}$, then in a $3.7 \%(\mathrm{v} / \mathrm{v})$ sodium hypochlorite and $0.1 \%$ Tween-20 solution for $35 \mathrm{~min}$, and lastly rinsed five times in sterile distilled water. Washed seeds were then transferred to sterile Petri dishes with autoclaved filter paper for drying.

\subsubsection{Culture media}

Fifteen rice seeds were plated in Petri dishes of callus induction media (MSP) (MS medium (Murashige and Skoog, 1962) (with Gamborg's B5 Vitamins, Duchefa) supplemented with 3\% (w/v) sucrose (Duchefa), $2.5 \mathrm{mg} / 1$ 2,4-D (Duchefa), 0.05\% (w/v) L-Proline (Duchefa), $0.03 \%$ (w/v) Casein hydrolysate (Duchefa) and solidified with $0.6 \%$ (w/v) Phytagel (Sigma-Aldrich)).

In subsequent steps the following media was used:

- Osmoticum media (MSO): MS medium (Murashige and Skoog, 1962) (with Gamborg's B5 Vitamins, Duchefa) supplemented with 3\% (w/v) sucrose (Duchefa), 7.82\% (w/v) D-Mannitol (Duchefa) $2.5 \mathrm{mg} / 1$ 2,4-D (Duchefa), 0.05\% (w/v) L-Proline (Duchefa), 0.03\% (w/v) Casein hydrolysate (Duchefa) and solidified with $0.3 \%$ (w/v) Phytagel (Sigma-Aldrich).

- Selection media (MSS): Callus induction media supplemented with $30 \mathrm{mg} / \mathrm{ml}$ hygromycin B (Duchefa).

- Shoot induction media (MSR): MS medium (Murashige and Skoog, 1962) (with Gamborg's B5 Vitamins, Duchefa) supplemented with 3\% (w/v) maltose (Duchefa), 0.01\% myo-inositol (Duchefa), $2 \mathrm{mg} / 1$ BAP (Sigma-Aldrich), $0.5 \mathrm{mg} / 1$ NAA (Sigma-Aldrich), 0.05\% (w/v) L-Proline (Duchefa), 0.03\% (w/v) Casein hydrolysate (Duchefa) and solidified with $0.25 \%$ (w/v) Phytagel (Sigma-Aldrich).

- Root induction media (MSR-R): Half strength MS medium (Murashige and Skoog, 1962) (with Gamborg's B5 Vitamins, Duchefa) supplemented with 3\% (w/v) sucrose (Duchefa), 0.01\% myo-inositol (Duchefa), 0.05\% (w/v) L-Proline 
(Duchefa), 0.03\% (w/v) Casein hydrolysate (Duchefa) and solidified with 0.25\% (w/v) Phytagel (Sigma-Aldrich).

The $\mathrm{pH}$ of the media was adjusted to 5.8 with $\mathrm{KOH}$ before autoclaving at $120^{\circ} \mathrm{C}$ for 18 min, except MRS, which was autoclaved for 8 min to avoid caramelization of maltose.

\subsubsection{Growth conditions}

Rice seeds and callus were grown in an in vitro growth chamber (Heratherm, Thermo Fisher Scientific) under dark conditions at $25^{\circ} \mathrm{C}$.

Rice callus in shoot or root induction media were grown in an environmentally controlled chamber (Sanyo) under 16/8 h light/dark conditions at $25^{\circ} \mathrm{C}$.

Regenerated plantlets that were transferred to soil grew in an environmentally controlled chamber (Conviron) under 16/8 h light/dark conditions at $24^{\circ} \mathrm{C}$ and $70 \% \mathrm{RH}$.

\subsubsection{Biolistic transformation}

Mature rice seeds were handled as described in Christou et al. (1991), Sudhakar et al. (1998) and Valdez (1998). Gold particles were coated with $20 \mu \mathrm{g}$ of pDNA. Level 1 pUC57 based transcriptional units containing eGFP fused to the individual chloroplast transit peptides, namely AtmCAB6тP, AtmTOCC ${ }_{\mathrm{TP}}$, AtmRCATP, and AtmBCCP1 $1_{\mathrm{TP}}$, were introduced separately into rice nature-embryo derived callus rice embryos, together with the $h p t$ gene for selection in a 3:1 ratio. Level 2 pUC57 based plasmids carried the nif genes of interest plus the hpt selectable marker.

Per each reaction, $10 \mathrm{mg}$ of gold particles of $0.76 \mu \mathrm{m}$ size were prepared in an Eppendorf tube. The pDNA was then added and mixed with $100 \mu 1$ XHO buffer, $100 \mu 10.1 \mathrm{M}$ spermidine, $100 \mu 1$ 25\% PEG 1300-1600, and $100 \mu 12.5 \mathrm{M} \mathrm{CaCl}_{2}$. After incubation for 10 min on vortex, the preparation was centrifuged and the pellet washed twice with $100 \%$ ethanol while sonicating briefly. The pDNA-coated gold particles were then resuspended in $9 \mathrm{ml}$ of $100 \%$ ethanol and stored at $-20^{\circ} \mathrm{C}$ until further use.

Six days-old rice embryos were excised, removing the expanded embryo from the seeds and incubating them on MSO for $4 \mathrm{~h}$ prior to bombardment with the scutellum facing upwards. Transformation of rice embryos with pDNA-coated gold particles was performed following Christou et al. (1991), in two bombardment steps at $15 \mathrm{kV}$ in a Helium atmosphere with $4 \mathrm{~h}$ interval. Subsequently, embryos were incubated overnight on MSO plates and transferred to fresh MSP for two days. Then, embryos were transferred to MSS plates and incubated for 14-15 days until embryogenic callus 
appeared, when they were transferred to fresh MSS plates (secondary selection) for verification of positive transformants. A minimum of two representative independent callus lines and the corresponding regenerated plants for each construct were selected for in-depth analyses.

\subsubsection{Plant regeneration}

After approximately $6-8$ weeks, positive embryogenic callus expressing the protein of interest were sequentially transferred to MSR plates and incubated in a 16/8 h photoperiod as described in Section 5.3.2.3.

Once the emerged shoots had sufficiently developed, tissue was carefully transferred to MSR-R and incubated as described in Section 5.3.2.3.

Regenerated plantlets with a sufficiently developed root system were transferred to $8 \times 8$ x $8 \mathrm{~cm}$ pots containing a 3:1 mixture of soil (Floragard) and vermiculite and were grown under flooded conditions in a growth chamber in a $12 / 12 \mathrm{~h}$ photoperiod at $28 / 25^{\circ} \mathrm{C}$ light/dark with constant $80 \%$ RH. Plants were irrigated with a soluble Fe solution (Sequestrene 138 Fe G-100, Syngenta) until seed maturity.

\section{Protein Techniques}

\subsection{Protein extraction}

\subsubsection{Preparation of Arabidopsis protoplast extracts}

A. thaliana transformed protoplasts were recovered from 96-well plates by gentle pipetting, transferred to $1.5 \mathrm{ml}$ Eppendorf tubes, and pelleted by centrifugation at $100 \times$ $g$ for $2 \mathrm{~min}$ (mesophyll cells) or $500 \times g$ for $10 \mathrm{~min}$ (root cells) at RT. Supernatants were discarded and pellets were shock frozen in liquid nitrogen. Protoplasts were resuspended in 2x Laemmli buffer (125 mM Tris-HCl pH 6.8, 4\% (w/v) SDS, 20\% (v/v) glycerol, 10\% $(\mathrm{v} / \mathrm{v}) \beta$-mercaptoethanol, $0.005 \%$ (w/v) bromophenol blue) and subsequently boiled for $10 \mathrm{~min}$, cooled down and centrifuged at $14,000 \times \mathrm{g}$ for $3 \mathrm{~min}$ at $4^{\circ} \mathrm{C}$ to obtain total protein extracts.

\subsubsection{Preparation of tobacco extracts}

Leaf discs from infiltrated $N$. benthamiana plants were harvested into 2-ml screw-cap tubes containing five 3-mm diameter glass beads and their weight was measured. Tubes were immediately frozen in liquid nitrogen and stored at $-80^{\circ} \mathrm{C}$ for further use. Tissue was ground to a fine powder using a BeadBug homogenizer (Benchmark Scientific Inc.) at maximum speed for $45 \mathrm{~s}$ avoiding thawing. Samples were mixed with 4 volumes of $2 \mathrm{x}$ 
Laemmli buffer, boiled for $10 \mathrm{~min}$, cooled down and centrifuged at $14,000 \times g$ for $3 \mathrm{~min}$ at $4^{\circ} \mathrm{C}$ to obtain total protein extracts.

\subsubsection{Chloroplast isolation assays}

Isolation of chloroplasts from agroinfiltrated leaves was performed as described in Klinkenberg (2014), with slight modifications. All steps were performed on ice. From 500 to $700 \mathrm{mg}$ of plant material was harvested and homogenized in a ratio 1:6 with icecold Chloroplast Isolation Buffer (CIB) (0.33 M Sorbitol, $50 \mathrm{mM}$ HEPES pH7.5, $1 \mathrm{mM}$ $\mathrm{MgCl}_{2}, 0.1 \%$ (w/v) BSA) using a precooled Potter homogenizer (Sigma-Aldrich).

After filtration through a $47 \mu \mathrm{m}$ nylon mesh (Merck) the intact chloroplasts were separated by centrifugation through a double Percoll (GE Healthcare Life Sciences) gradient $(40 / 80 \%)$ at $3,200 \times g$ at $4^{\circ} \mathrm{C}$ for $15 \mathrm{~min}$. The band that formed at the interface was collected and washed twice with CIB without BSA. Chloroplasts were shock frozen in liquid nitrogen, resuspended in ice-cold protein extraction buffer (100 mM HEPES pH 7.2, $1 \mathrm{mM} \mathrm{MgCl} 2,10 \mathrm{mM}$ DTT, 10\% (w/v) glycerol, 0.5\% (v/v) Protease Inhibitor Cocktail) and centrifuged at $15,000 \times \mathrm{g}$ at $4^{\circ} \mathrm{C}$ for $10 \mathrm{~min}$. The supernatant "stroma fraction (S)" was recovered and the "pellet fraction (P)", was re-suspended in the same volume of protein extraction buffer.

\subsubsection{Preparation of rice extracts 6.1.3.1. Rice protoplasts}

Rice transformed protoplasts were recovered from 96-well plates by gentle pipetting, transferred to $1.5 \mathrm{ml}$ Eppendorf tubes, and pelleted by centrifugation at $300 \times \mathrm{g}$ for $6 \mathrm{~min}$ at RT. Supernatants were discarded and pellets were shock frozen in liquid nitrogen. Protoplasts were resuspended in 2x Laemmli buffer and subsequently boiled for $10 \mathrm{~min}$, cooled down, and centrifuged at $14,000 \times g$ for $3 \mathrm{~min}$ at $4^{\circ} \mathrm{C}$ to obtain total protein extracts.

\subsubsection{Rice callus}

Rice callus was harvested and placed into $1.5 \mathrm{ml}$ Eppendorf tubes, weighted, immediately frozen in liquid nitrogen, and then stored at $-80^{\circ} \mathrm{C}$ until further use. Callus tissue was homogenized in the presence of ice-cold protein extraction buffer composed of $100 \mathrm{mM}$ HEPES pH 7.2, 1 mM MgCl $2,10 \mathrm{mM}$ DTT, 10\% (w/v) glycerol, and 0.5\% (v/v) Protease Inhibitor Cocktail (Sigma-Aldrich) with the help of a stainless-steel pestle, and subsequently centrifuged at $14,000 \times g$ for $3 \mathrm{~min}$ at $4^{\circ} \mathrm{C}$ to obtain soluble protein extracts. 


\subsubsection{Rice leaves and roots}

Rice leaf and root tissue samples were excised from plantlets when transferring them from Magenta GA-7 vessels (Magenta Corp.) to soil and harvested into 2-ml screw-cap tubes containing five 3-mm diameter glass beads, weighted, immediately frozen in liquid nitrogen, and then stored at $-80^{\circ} \mathrm{C}$ until further use. Samples were ground to a fine powder with the help of a plastic pestle and a BeadBug homogenizer (Benchmark Scientific Inc.), mixed with 4 volumes of $2 x$ Laemmli buffer, boiled for $10 \mathrm{~min}$, cooled down, and centrifuged at $14,000 \times g$ for $3 \mathrm{~min}$ at $4^{\circ} \mathrm{C}$ to obtain total protein extracts.

\subsection{Immunoblot assays}

SDS-PAGE (Laemmli, 1970) and immunoblot (Towbin et al., 1979) analysis were performed by standard methods. Samples processed with protein extraction buffer were resuspended in Laemmli sample buffer $2 x$, where total protein extracts were used directly. Samples were loaded in $12 \%$ electrophoresis polyacrylamide gels and run on a MiniProtean Tetra cell (Bio-Rad). SDS gels were either stained with Coomassie $(0.1 \%(\mathrm{w} / \mathrm{v})$ Coomassie R-250 (Thermo Fisher Scientific), 40\% ethanol, 10\% glacial acetic acid) or electroblotted to $0.45 \mu \mathrm{m}$ pore-size nitrocellulose membranes (Bio-Rad) using a TransBlot Semi-Dry Transfer cell (Bio-Rad).

Blotted membranes were stained with Ponceau S solution $(0.2 \%(\mathrm{w} / \mathrm{v})$ Ponceau S (SigmaAldrich) (w/v) in 5\% glacial acetic acid) for quick checking of correct protein transfer and subsequently distained with TBS-T (pH 7.5, $200 \mathrm{mM}$ Tris-base, $1.5 \mathrm{M} \mathrm{NaCl}, 0.2 \%$ (v/v) Tween-20).

For the immunodetection of proteins, primary antibodies prepared in $5 \%(\mathrm{w} / \mathrm{v}) \mathrm{BSA}$ and $0.1 \%(\mathrm{v} / \mathrm{v}) \mathrm{NaN}_{3}$ were used as follows: in-house generated rabbit polyclonal antibodies detecting NifH (1:3500 dilution) (López-Torrejón et al., 2016), NifU (1:2000 dilution) (López-Torrejón et al., 2016), NifS (1:1200 dilution) (López-Torrejón et al., 2016) and NifM (1:2500 dilution) (Burén, Young, et al., 2017) were used. Commercially available antibodies against GFP (1:3000 dilution) (11814460001; Roche), Strep-tag II (1:10000 dilution) (Strep-Tactin-HRP, 2-1502-001; IBA Lifesciences), RbcL (1:10000 dilution) (AS03 037; Agrisera AB), Actin-11 (1:2000 dilution) (AS10 702; Agrisera AB) and IDH (1:5000 dilution) (AS06 203A; Agrisera AB) were used. Secondary horseradish peroxidase-conjugated antibodies were prepared in $2 \%(\mathrm{w} / \mathrm{v})$ skimmed milk as follows: anti-rabbit IgG (1:25000 dilution) (A0545-1ML; Sigma-Aldrich) and anti-mouse IgG (1:10000 dilution) (AS11 1772; Agrisera AB). 
Immunoblots were developed in an iBright FL1000 Imaging System (Thermo Fisher Scientific) using chemiluminescence mode. Smart exposure setting was used to develop blots and iBright Analysis Cloud Service (Thermo Fisher Scientific) was used for image processing, which was equally applied across the entire image including control lines.

\subsubsection{Quantification of Immunoblot data}

For protein accumulation measurements, short exposure blots were analysed in iBright Analysis Cloud Service (Thermo Fisher Scientific). The signal intensity for each band was measured using automatic band recognition and background subtraction.

\subsubsection{Statistical analysis}

The accumulation values for each Nif protein normalized using GFP signal shown in Figures 9, 10 and 19 were graphically represented using Prism (GraphPad). Significant differences in Nif protein expression were determined by applying a Student's t-test or Tukey's multiple comparisons test, where a p-value $<0.05$ was considered statistically significant and a p-value $<0.01$ highly significant.

The import efficiency values for each TP shown in Figures 23, 25 and 27 were graphically represented using Prism (GraphPad) and significant differences in eGFP protein import efficiency between tissues were determined by applying a Student's t-test, where a pvalue $<0.05$ was considered statistically significant.

\subsection{Anaerobic purification of TwinStrep-tagged $\mathrm{NbNifH}$}

Agroinfiltrated $N$. benthamiana fresh leaves were ground in liquid nitrogen in the presence of quartz sand and resuspended in anaerobic lysis buffer $(100 \mathrm{mM}$ Tris- $\mathrm{HCl} \mathrm{pH}$ 8.0, $250 \mathrm{mM} \mathrm{NaCl}, 2 \mathrm{mM}$ sodium dithionite (DTH)) supplemented with $1 \mathrm{mM}$ phenylmethylsulfonyl fluoride (PMSF), $1 \mathrm{mM}$ leupeptin, and $5 \mu \mathrm{g} / \mathrm{ml}$ DNaseI. After blender homogenization, the extract was filtered through a cloth, then centrifuged at $73,000 \times \mathrm{g}$ for $1 \mathrm{~h}$ at $4^{\circ} \mathrm{C}$ under anaerobic conditions, and the supernatant filtered through a Nalgene filter unit (Thermo Fisher Scientific) with $0.2 \mu \mathrm{m}$ pore size, resulting in the cell-free extract (CFE).

Twin-Strep-tag $\mathrm{NbNifH}$ was purified by Strep-Tactin XT affinity chromatography under anaerobic conditions $(<0.1 \mathrm{ppm}$ oxygen) using an AKTA Prime FPLC system (GE Healthcare Life Sciences) inside an MBraun glovebox (M. Braun Inertgas-Systeme $\mathrm{GmbH}$ ). All buffers were previously made anaerobic by sparging with nitrogen gas. The CFE was loaded at $2 \mathrm{ml} / \mathrm{min}$ into a $5 \mathrm{ml}$ Streptactin-XT high capacity cartridge (IBA Lifesciences) previously equilibrated with lysis buffer, then washed with 20 column 
volumes of lysis buffer and eluted with 6 column volumes of elution buffer ( $50 \mathrm{mM}$ Tris$\mathrm{HCl} \mathrm{pH}$ 8.0, $250 \mathrm{mM} \mathrm{NaCl}, 5 \%$ glycerol, $2 \mathrm{mM} \mathrm{DTH}, 50 \mathrm{mM}$ biotin). The elution fraction was concentrated using an Amicon Ultra Centrifugal Filter unit (Amicon Ultra-15, Merck) with a cut-off pore size of $30 \mathrm{kDa}$, and then desalted using a PD-10 column (GE Healthcare Life Sciences) equilibrated in buffer (50 mM Tris-HCl pH 8.0, $250 \mathrm{mM} \mathrm{NaCl}$, $5 \%$ glycerol, $2 \mathrm{mM} \mathrm{DTH}$ ) in order to remove the biotin. Purified $\mathrm{NbNifH}$ was frozen and stored in cryogenic tubes (Nalgene) in liquid nitrogen until used for acetylene reduction assays.

\subsection{Edman Degradation}

Three $\mu \mathrm{g}$ of pure $\mathrm{NbNifH}$ were resolved by SDS-PAGE and blotted to an Immun-Blot PVDF membrane (pore size $0.45 \mathrm{~mm}$, Bio-Rad) using blot buffer ( $50 \mathrm{mM}$ sodium borate $\mathrm{pH} 9.0,20 \%$ methanol, and $0.1 \% \mathrm{SDS}$ ) at $100 \mathrm{~mA}$ for $12 \mathrm{~h}$, and $400 \mathrm{~mA}$ for 3 additional $\mathrm{h}$. The membrane was then stained with Coomassie Brilliant Blue R250 (Sigma-Aldrich) and dried. Afterwards, a single band corresponding to $\mathrm{NbNifH}$ was excised and submitted to Proteome Factory AG for sequence analysis. An ABI Procise 491 Protein Sequencer (Applied Biosystems) was used for automated N-terminal sequencing according to the manufacturer's protocol.

\subsection{Peptide mass fingerprinting}

SDS-PAGE of $10 \mu \mathrm{g}$ total protein from the concentrated $N b \mathrm{NifH}$ fraction was run and stained with Coomassie Brilliant Blue R250 (Sigma-Aldrich). As shown in Supplemental Figure 1, bands corresponding to supposed $\mathrm{NbNifH}$, contaminants, and degradation products were manually excised from gels and stored in Milli-Q $\mathrm{H}_{2} \mathrm{O}$ until analysis. The proteomic analysis was performed by the Proteomics Unit of CAI Técnicas Biológicas (Universidad Complutense de Madrid), as described below.

Proteins selected for analysis were in-gel reduced, alkylated, and digested with trypsin according to Sechi and Chait, 1998. Briefly, the samples were reduced with $10 \mathrm{mM}$ dithioerythritol in $25 \mathrm{mM}$ ammonium bicarbonate for $30 \mathrm{~min}$ at $56^{\circ} \mathrm{C}$ and subsequently alkylated with $25 \mathrm{mM}$ iodoacetamide in $25 \mathrm{mM}$ ammonium bicarbonate for $15 \mathrm{~min}$ in the dark. Finally, samples were digested with $12.5 \mathrm{ng} / \mu 1$ sequencing grade trypsin (Roche) in $25 \mathrm{mM}$ ammonium bicarbonate $(\mathrm{pH} 8.5)$ overnight at $37^{\circ} \mathrm{C}$.

After digestion, the supernatant was collected and $1 \mu 1$ was spotted onto a MALDI target plate and allowed to air-dry at RT. Then, $0.6 \mu 1$ of a $3 \mathrm{mg} / \mathrm{ml}$ of $\alpha$-cyano-4hydroxycinnamic acid matrix (Sigma-Aldrich) in 50\% acetonitrile was added to the dried peptide digest spots and allowed again to air-dry at RT. 
MALDI-TOF MS analyses were performed in a 4800 Plus Proteomics Analyzer MALDITOF/TOF mass spectrometer (Applied Biosystems). The MALDI-TOF/TOF operated in positive reflector mode with an accelerating voltage of 20,000 V. All mass spectra were calibrated internally using peptides from the autodigestion of trypsin.

For protein identification SwissProt DB 2016_10 (552884 sequences; 197760918 residues) without taxonomy restriction and Home DB were searched using MASCOT 2.3 (www.matrixscience.com) through the software Global Protein Server v 3.6 (ABSciex). Search parameters were:

- Carbamidomethyl Cysteine as fixed modification and oxidized methionine as variable modification

- Peptide mass tolerance, 50 ppm (PMF)

- 1 missed trypsin cleavage site

In all protein identification, the probability scores were greater than the score fixed by mascot as significant with a $p$-value $<0.05$.

\subsection{Fe protein activity determination}

Fe protein activity of $\mathrm{NbNifH}$ preparations was analysed by the acetylene to ethylene reduction assay. Fe protein was added to a $600 \mu \mathrm{lml}$ reaction mixture containing $13 \mu \mathrm{M}$ MoFe protein (Fe protein to MoFe protein molar ratio 25:1) and an ATP-regenerating mixture (1.23 mM ATP, $18 \mathrm{mM}$ phosphocreatine, $2.2 \mathrm{mM} \mathrm{MgCl} 2,3 \mathrm{mM} \mathrm{DTH}$, and 40 $\mu \mathrm{g}$ of creatine phosphokinase, $22 \mathrm{mM}$ Tris- $\mathrm{HCl} \mathrm{pH} 7.5$ ) in a $9 \mathrm{ml}$ serum vial sealed with a rubber stopper and flushed with $100 \%$ argon. After injection of $500 \mu 1$ acetylene (Contse S.A.) the vial was incubated at $30^{\circ} \mathrm{C}$ for 15 min with shaking (Shah and Brill, 1973). The reaction was stopped by adding $100 \mu \mathrm{l}$ of $8 \mathrm{M} \mathrm{NaOH}$.

Ethylene formation was measured in a Shimadzu GC-2014 gas chromatograph (Shimadzu Corporation) equipped with a PoraPak N 80/100 column (Agilent Technologies). Positive control reactions were carried out with Fe protein and MoFe proteins purified from $A$. vinelandii under anaerobic conditions as described by Curatti et al. (2007).

\subsection{Microscopy Techniques}

\subsubsection{Light-Microscopy}

Protoplast from Arabidopsis and rice cells were placed on a Neubauer counting chamber (Marienfeld-Superior) and imaged using a Zeiss AxioPhot Microscope (Zeiss) equipped 
with a Plan-Neofluar 40X/0.75 objective, coupled to a colour CCD Leica DFC 300FX camera (Leica) and LAS software. Bright Field images were processed with LAS software and assembled using Illustrator software (Adobe).

\subsubsection{Stereomicroscopy}

Rice callus was imaged using a Fluorescent Stereo Microscope Leica MZ10 F (Leica) equipped with a Plan-Apochromat 1.0x/0.125 objective and Leica Application Suite (LAS) v4.3 software (Leica). A GFP Plus filter was set with the following parameters to detect eGFP: excitation wavelength 460 - $500 \mathrm{~nm} / \mathrm{emission} 510 \mathrm{~nm}$. Images were processed with LAS software and assembled using Illustrator software (Adobe).

\subsubsection{Confocal Microscopy}

Samples were imaged using a Zeiss LSM880 confocal laser scanning microscope (Zeiss) equipped with a Plan-Apochromat 40X/1.2 water-immersion or Plan-Apochromat 63X/1.2 oil-immersion objective and ZEN 2.6 Black software (Zeiss). The excitation laser lines wavelength/emission bands used with PMT detectors were as follows: GFP (488 nm/493 to $556 \mathrm{~nm})$, BFP (405 nm/410 to $529 \mathrm{~nm})$, mCherry $(561 \mathrm{~nm} / 595$ to 640 $\mathrm{nm})$, and chlorophyll autofluorescence $(633 \mathrm{~nm} / 647$ to $721 \mathrm{~nm})$. To avoid overlap between the fluorescence channels, sequential scanning was used when necessary. Superresolution (AS-SR) imaging was performed with an Airyscan detection unit, sequentially, with excitation as described above and with emission band-pass filters as follows: GFP (488 nm/BP 420-480 + BP 495-550), mCherry (BP 420-480 + BP 495-620), and chlorophyll autofluorescence (633 nm/ BP 570-620 + LP 645).

ZEN 2.6 Black software (Zeiss) was used to process the AS-SR acquired datasets with automatically-determined Airyscan Filtering. The software processes each of the 32 Airy detector channels by performing filtering, deconvolution, and pixel reassignment to obtain images with enhanced spatial resolution and improved signal-to-noise ratio (SNR) (Huff, 2015). Images were post-processed with ZEN 2.6 Blue (Zeiss) and assembled using Illustrator software (Adobe).

\subsubsection{Tobacco leaves}

Leaf discs from 4 to 5-week-old plants were excised and mounted onto microscope slides (Thermo Fisher Scientific) in a PBS solution using High-Performance cover glasses (Zeiss). 


\subsubsection{Arabidopsis and rice protoplasts}

Protoplasts were resuspended with gentle pipetting and a small drop mounted onto a microscope slide inside an observation gel. A High-Performance cover glass (Zeiss) was placed on top of the sample. For protoplast image post-processing, Z-axis maximum intensity projections were performed in ZEN 2.6 (Zeiss).

\subsubsection{Rice leaves}

Leaf tissue samples $(1 \times 10 \mathrm{~mm})$ were fixed with $2 \%(\mathrm{w} / \mathrm{v})$ paraformaldehyde in $0.1 \mathrm{M}$ sodium phosphate buffer ( $\mathrm{pH} 7.2)$ and cut into semithin sections $(30-40 \mu \mathrm{m})$ using a CM3050S Research Cryostat (Leica Microsystems). The sections were collected on standard glass microscope slides pre-coated with poly-L-lysine and images were captured using an FV1000 laser scanning confocal microscope (Olympus) with illumination at 488 $\mathrm{nm}$ (excitation wavelength of eGFP, multiline argon laser).

\subsubsection{Immuno-electron microscopy}

For analysis of rice tissues, samples were sent to the Servei de Microscòpia (Universitat de Lleida) and Servicio Central de Soporte a la Investigación Experimental (Universidad de Valencia) for preparation and analysis respectively. A detailed protocol is detailed below.

For electron microscopy immune detection, pieces of rice callus $\left(1 \mathrm{~mm}^{3}\right)$ or leaf tissue (1 x $10 \mathrm{~mm})$ or root $(10 \mathrm{~mm})$ were fixed with $1 \%(\mathrm{w} / \mathrm{v})$ glutaraldehyde and $1 \%(\mathrm{w} / \mathrm{v})$ paraformaldehyde in $0.1 \mathrm{M}$ sodium phosphate buffer $(\mathrm{pH} 7.2)$ for $16-24 \mathrm{~h}$ at $4^{\circ} \mathrm{C}$. To enhance the penetration of fixative, samples were initially subjected to a light vacuum until the tissue pieces sank. Once fixed, pieces were washed three times $(10 \mathrm{~min})$ with the same buffer and dehydrated in an ethanol series $(30-100 \%)$ before embedding in Lowicryl K4 $\mathrm{M}$ resin (Polysciences) in a cold chamber at $-20^{\circ} \mathrm{C}$ to $-35^{\circ} \mathrm{C}$ and inducing polymerization by exposure to ultraviolet light.

Semithin $(2 \mu \mathrm{m})$ and ultrathin $(70-90 \mathrm{~nm})$ sections were prepared using a Reichert-Jung ultra-cut E cryotome (Leica). The semithin sections were stained with Richardson's blue, covered with a drop of DPX slide mounting medium and a coverslip, and observed under a DM4000B microscope (Leica). Images were captured using a DFC300 FX 1.4-MP digital colour camera equipped with LAS v3.8 (Leica). The ultrathin sections were mounted on Formvar carbon-coated gold grids (200 mesh) and incubated for $30 \mathrm{~min}$ in blocking buffer for polyclonal antibodies $(200 \mathrm{mM}$ Tris- $\mathrm{HCl}$ at $\mathrm{pH} 7.4,1 \%$ Tween-20, $0.1 \%$ gelatin $(\mathrm{w} / \mathrm{v}), 1 \% \mathrm{BSA}(\mathrm{w} / \mathrm{v}))$ or monoclonal antibodies $(10 \mathrm{mM}$ Tris- $\mathrm{HCl}$ at $\mathrm{pH}$ 
7.4, 0.9\% NaCl, 0.05\% PEG 20,000, 3\% BSA). The grids were then washed in distilled water and incubated overnight at $4{ }^{\circ} \mathrm{C}$ with primary polyclonal anti-eGFP antibody PA522688 (Thermo Fisher Scientific) diluted 1:200 in blocking buffer, or primary monoclonal anti-eGFP antibody 11814460001 (Sigma-Aldrich) diluted 1:250 in blocking buffer. After washing in distilled water, followed by a further 30-min incubation in the appropriate blocking buffer and another wash, the grids were incubated at RT for $1 \mathrm{~h}$ with the 15-nm gold-conjugated secondary antibody diluted 1:20 in the appropriate blocking buffer:goat-anti-rabbit IgG for the polyclonal antibody, or EM-grade goat-anti-mouse $\mathrm{IgG}$ for the monoclonal antibody (Electron Microscopy Sciences). Finally, the grids were contrasted with $1 \%(\mathrm{w} / \mathrm{v})$ uranyl acetate in water $(20 \mathrm{~min})$ and Reynold's lead citrate (2 min) before observation in Jeol Jem-1010 Transmission Electron Microscope (Jeol Ltd.). A minimum of two grids per treatment and sample were analysed. 


\section{Results}

7. Use of synthetic biology tools to optimize the production of active nitrogenase Fe protein in chloroplasts of tobacco leaf cells ${ }^{\dagger}$

\subsection{Introduction}

Nitrogen is a critically important element for all organisms, the element that is required in amounts greater than all others except for carbon, oxygen, and hydrogen. It is very abundant in the atmosphere, which is composed of $78 \%$ molecular nitrogen gas; however, neither plants nor animals can obtain nitrogen directly from dinitrogen. Instead, they ultimately depend on a process performed by some archaea and bacteria known as biological nitrogen fixation, where dinitrogen is converted into usable ammonia by the nitrogenase enzyme.

Nitrogen availability often limits the productivity of natural and managed ecosystems, so much so that among all farmer-controlled input factors nitrogen is considered to have the second-largest impact on plant growth, only after water (Martin and Marschner, 1988). To increase crop yield, the use of nitrogen fertilizers has become widespread in developed countries, with enormous undesired environmental consequences that cannot be overlooked. On the other hand, the cost of chemical fertilizers is prohibitive for farmers in the developing countries, which is why there are regions in Sub-Saharan Africa and Southeast Asia where crop yields are extremely low and poverty and hunger prevail (Mueller et al., 2012).

The use of biotechnology to boost biological nitrogen fixation has been considered, for at least the last 45 years, as one of the best strategies to increase crop yield to face population growth and general agronomic crop demand while preserving the environment (Beatty and Good, 2011; Burén and Rubio, 2018; Curatti and Rubio, 2014; Hardy and Havelka, 1975; Ladha and Reddy, 1995; Oldroyd and Dixon, 2014; Rogers and Oldroyd, 2014; Rosenblueth et al., 2018). In this context, one of the proposed biotechnological strategies involves the direct transfer of the prokaryotic genes needed to assemble a functional molybdenum nitrogenase (nif genes) into the plant genome, a huge undertaking given the relatively large number of genes in the nitrogenase biosynthetic pathway and

\footnotetext{
$\dagger$ This section has been partially published in Plant Biotechnology Journal:

Eseverri, Á., López-Torrejón, G., Jiang, X., Burén, S., Rubio, L.M., and Caro, E. (2020) Use of synthetic biology tools to optimize the production of active nitrogenase Fe protein in chloroplasts of tobacco leaf cells. Plant Biotechnol. J., 18, 1882-1896. doi: 10.1111/pbi.13347
} 
the extreme oxygen-sensitivity of nitrogenase structural components and the proteins involved in the synthesis of their metal cofactors (Curatti and Rubio, 2014). The molybdenum nitrogenase is composed of an Fe protein (encoded by nifH) and a MoFe protein (encoded by nifD and nifK), which require [Fe-S] clusters for activity. Since the nitrogenase Fe protein is the most sensitive component to oxygen, and its maturation requirements are simpler and better characterized than those of the MoFe protein, it has been chosen as a proof of concept by most previous efforts to express nif genes in eukaryotes. There are four genes in $A$. vinelandii required to render active Fe protein (nifH, nifM, nifU, and nifS) (Jacobson et al., 1989). NifM is a putative peptidyl-prolyl cistrans isomerase that assists in the proper folding of NifH (Gavini et al., 2006). NifS is a cysteine desulfurase responsible for the mobilization and transfer of sulfur to NifU (Yuvaniyama et al., 2000; Zheng et al., 1993), where [Fe-S] clusters are synthesized and later donated to the cofactor-less apo-Fe protein for activation (Dos Santos et al., 2004). These are oxygen-sensitive processes in which intermediates are generated and carried directly from one protein to another to protect them from reactive species (Zheng and Dos Santos, 2018).

The most successful efforts in transferring active nitrogenase components to eukaryotes to date have been the production of active NifU and NifH within the mitochondria of yeast cultures growing aerobically, overcoming the oxygen-sensitivity of Nif proteins (López-Torrejón et al., 2016), and the production of active NifB and its product NifB-co which are key to the biosynthesis of the Mo-nitrogenase active-site cofactor, called FeMo-co (Burén et al., 2019). In addition, Allen et al. (2017) showed that indeed, 16 nifor nif-related genes from $K$. oxytoca could be individually expressed fused to mitochondrial targeting peptides to accumulate in the mitochondrial matrix of tobacco cells. However, no protein activity was demonstrated.

A different location where to engineer nitrogen fixation in plants is chloroplasts, suggested already in 1984 by Merrick and Dixon because of their availability of the immediate products of photosynthesis, NADPH, and ATP, that could satisfy nitrogen fixation demands of energy and reducing power. Moreover, the ammonia produced as a consequence of nitrogen fixation could be easily assimilated into the formation of amino acids by the glutamine synthetase/glutamate synthase pathway, found in plastids. In 1997, Dixon et al. expressed nifH and nifM in tobacco leaf chloroplasts. Even though it was shown that the proteins were stable when targeted to this organelle by transit peptides, they accumulated at levels too low for functionality tests. Using a different strategy, Cheng et al. (2005) transformed directly the chloroplast genome and showed that NifH was expressed and accumulated in the chloroplasts of the unicellular alga 
Chlamydomonas reinhardtii. Although Fe protein activity was not proven, it could partially substitute for the function of the putative 'chlorophyll iron protein' encoded by chlL, a protein similar to NifH in both structure and function and also sensitive to oxygen. More recently, Ivleva et al. (2016) reported the generation of transplastomic tobacco plants expressing nifH and nifM. Their approach rendered a high amount of protein accumulating in the leaves of the plants, but NifH activity could only be detected when plants were incubated under low oxygen conditions (10\% oxygen), and even then, the activities obtained were very low.

Thus, although it has been possible to express some nitrogenase proteins in chloroplasts, there are still problems to overcome. While direct transformation of the chloroplast would be ideal (Adem et al., 2017), since chloroplast transformation methods are not yet in place for maize and very few successful applications have been developed for rice, we have focused our work on the expression of nuclear-encoded nif genes. In this context, increasing nif gene expression and optimizing the import of the recombinant proteins into chloroplasts were our main priorities in the pursuit of active Fe protein in chloroplasts using a strategy that can be directly transferred to cereals.

It is well known that the DNA sequence used to encode a protein can have dramatic effects on its expression and accumulation (Gustafsson et al., 2012). mRNA structures near the ribosome binding site were long ago reported to reduce translational initiation (Kozak, 1986), as well as mRNA destabilization by the basal mRNA decay machinery and sequence-specific decay components (Gutiérrez et al., 1999). The exclusion of these elements from synthetic genes, together with premature polyadenylation sites and cryptic intron splice sites, has been described to increase transcription and enhance translation in plants (Jackson et al., 2014). In an attempt to design synthetic nif genes with a higher expression and stability, we eliminated these undesired elements from their sequence and had them match codon usage and GC content from the host as much as possible, which is well known to affect recombinant protein expression (Webster et al., 2017).

Another step that could be limiting the availability of Nif proteins in the chloroplast is their import from the cytosol. It requires chloroplast transit peptides (TPs), N-terminal targeting sequences that direct proteins to their correct subcellular compartment and, after import through an ATP hydrolysis-dependent process, are cleaved by the stromal processing peptidase. The transit peptide of the small subunit of RuBisCo, the most commonly used TP, is very efficient in driving most recombinant proteins to the chloroplast stroma, although the N-terminal region of cargo proteins is also critical for TP activity (Shen et al., 2017). 
In the work presented here, the generation of synthetically designed genes to maximize Nif protein accumulation and the screening for the optimal chloroplast transit peptide for each Nif protein led to tobacco plants where NifH, M, U, and S accumulated as soluble proteins within the stroma of mesophyll cells and Fe protein activity could be shown for $\mathrm{NifH}$.

\subsection{Results}

\subsection{1. nif gene synthetic design}

Previous studies had shown that nuclear-encoded nifH could not be expressed to high levels in tobacco chloroplasts using its original sequence from $K$. oxytoca (Dixon et al., 1997). The codon adaptation index (CAI) is a simple and effective way of measuring synonymous codon usage bias and the likeliness of success of heterologous gene expression (Sharp and $\mathrm{Li}, 1987$ ). The sequences of $A$. vinelandii nifH, nifM, nifU, and nifS codon-optimized for their expression in S. cerevisiae (López-Torrejón et al., 2016) showed theoretical good optimization also for expression in tobacco, with a CAI of 0.82 , $0.84,0.81$, and 0.82 , respectively, and no presence of low frequency $(<40 \%)$ codons (Biologics International Corp codon adaptation index calculator and rare codon analyser). These values strongly indicated that heterologous gene expression in tobacco using the yeast codon-optimized sequences could be successful, especially when compared with the data for original non-optimized $A$. vinelandii genes (CAIs of $0.60,0.54,0.55$, and 0.53 and presence of 5, 16, 10, and 12 low-frequency codons for nifH, nifM, nifU, and nifS, respectively).

We decided to design nif synthetic genes specifically optimized for maximum expression in tobacco taking into account, not only codon usage patterns, but also additional parameters such as mRNA stability motifs, hidden stop codons and poly(A) signals that could impact protein production. The web server application Codon Optimization OnLine (COOL) (Chin et al., 2014) was used for this purpose.

The specific parameters of the synthetic design used in this work involved using an individual codon usage (ICU) and a GC content matching that of tobacco coding sequences, both overall and specifically in the third base of codons (GC3) (Nakamura et $a l ., 2000)$. Moreover, some motifs were actively avoided, like any form of dicotyledonous plant polyadenylation signal, reported RNA instability motifs and repetitions of more than seven bp or A and T strings (Jackson et al., 2014; Ji et al., 2007; Shen et al., 2008) (Supplemental Table 5). Synthetic designed nif sequences closely matched the ICU and GC3 content of tobacco compared to the yeast codon-optimized sequences. Furthermore, exclusion motifs and repetitions were effectively removed to a large degree. In contrast, 
CAIs values pointed to slightly better performance of yeast codon-optimized sequences (Supplemental Table 6).

(A)

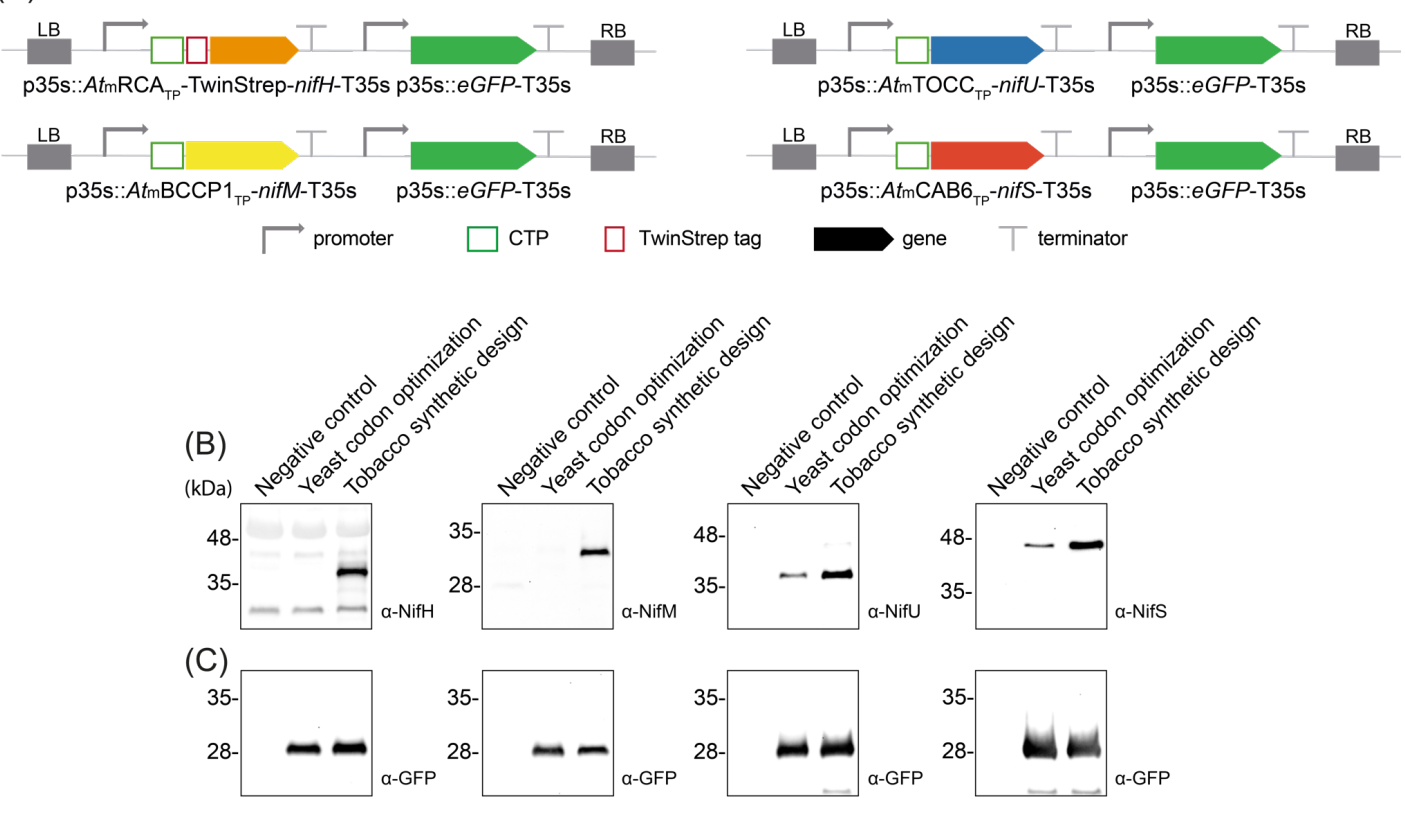

(D)

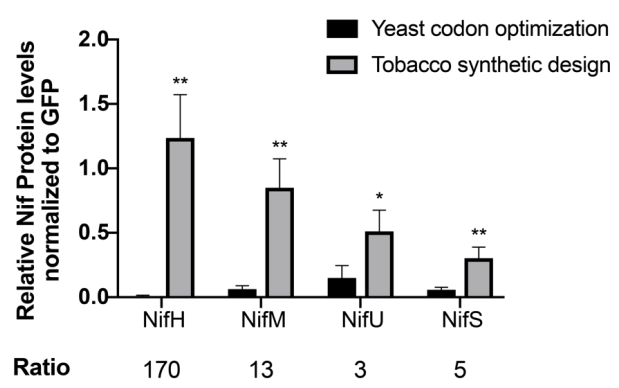

Figure 9. Effect of using synthetically designed nif genes optimized for tobacco.

(A) Schematic representation of the constructs used in N. benthamiana-transient expression assays leading to nif genes and eGFP simultaneous expression. (B) Western blot analysis of NifH, NifM, NifU, and NifS accumulation when expressed from yeast codon-optimized genes or synthetically designed genes optimized for expression in tobacco. Negative control refers to $N$. benthamiana leaves agroinfiltrated with Agrobacterium transformed with an empty vector. Expected sizes are Twin-Strep NifH: $35.3 \mathrm{kDa}$, NifM: $32.3 \mathrm{kDa}$, NifU: $34.4 \mathrm{KDa}$, and NifS: $44.3 \mathrm{kDa}$. (C) Western blot analysis of eGFP accumulation. Expected size: $27 \mathrm{kDa}$. (D) Quantification of Nif/eGFP accumulated protein. Mean $\pm \mathrm{SD}$ ( $\mathrm{n}=3$ biological replicates). The given value for each Nif protein band was normalized using the value corresponding to the eGFP signal in the same lane. 'Ratio' refers to the ratio between Nif protein levels achieved using the tobacco synthetic design and the yeast codon-optimized sequences. * Represents Student's test significant differences $(P<0.05)$;** represents Student's test highly significant differences $(P<0.01)$.

We generated multigenic constructs comprised of a transcriptional unit where the p35S promoter directs the expression of each yeast codon-optimized or synthetically designed nif gene and a second transcriptional unit where the $\mathrm{p} 35 \mathrm{~S}$ directs the expression of eGFP. Transient expression assays were performed to gauge the effect of the synthetic gene 
design (Figure 9A). The accumulation of Nif proteins (Figure 9B) was compared, using the level of eGFP to normalize for differences in overall recombinant protein expression (Figure 9C). The results showed increased accumulation of all four Nif proteins when using the constructs with the synthetically optimized genes, validating the parameters established for their re-design (Figure 9D). The increase was specifically significant in the case of $\mathrm{NifH}$, which accumulated 170 times more when the synthetic gene was used for expression (Figure 9D).

(A)

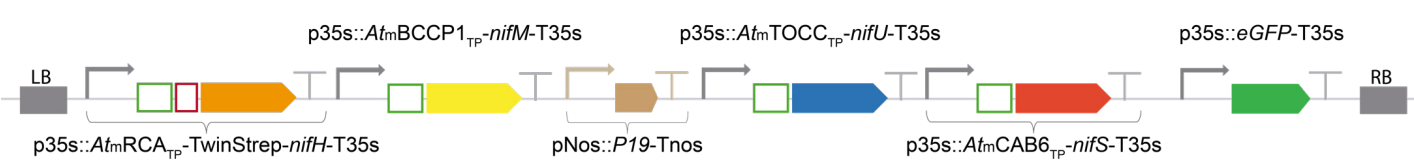

(B)

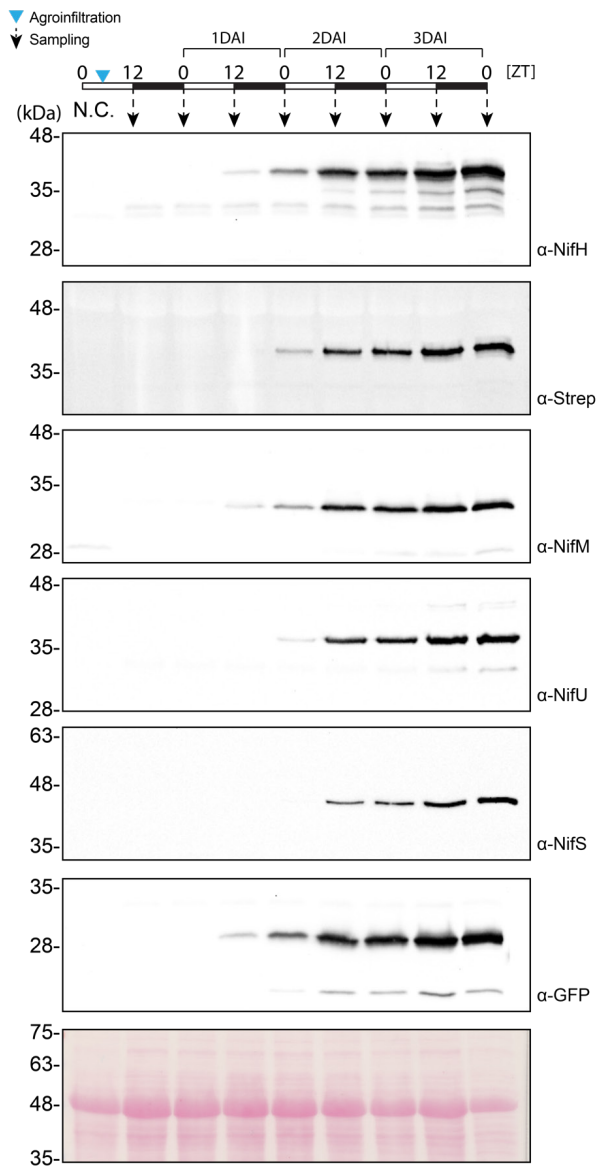

(C)

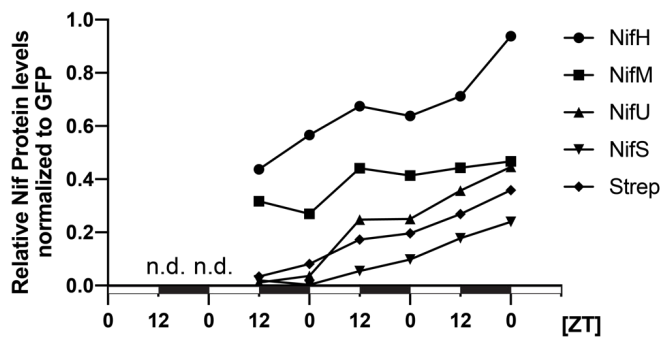

Figure 10. Time course accumulation of synthetically designed nif genes optimized for tobacco.

(A) Schematic representation of the construct used in $N$. benthamiana-transient expression assays leading to nif genes and eGFP simultaneous expression. (B) Time course of NifH (tobacco synthetic design) and eGFP accumulation after $N$. benthamiana agroinfiltration. N.C. refers to 'negative control', $N$. benthamiana leaves agroinfiltrated with Agrobacterium transformed with an empty vector. Expected sizes are Twin-Strep NifH: 35.3 kDa, NifM: 32.3 kDa, NifU: 34.4 KDa, NifS: 44.3 kDa, and eGFP: 27 kDa. (C) Quantification of Nif/eGFP accumulated protein. 'n.d.' refers to 'non-detected'. 
We followed the accumulation of NifH, NifM, NifU, and NifS through a time-course from the moment of agroinfiltration to optimize the time of tissue collection, and found that protein levels steadily increased until the end of the dark period of the third day (Figure 10). This is in accordance with the standard method for transient expression in $N$. benthamiana (Li, 2011; Naim et al., 2012), and shows that the oxygen derived from photosynthesis taking place during the light period of plant growth did not lead to a decrease in NifH polypeptide levels or any of the other Nif proteins accumulation.

\subsubsection{Optimization of chloroplast targeting}

In an effort to find the TP that best suited each specific Nif protein in study, 12 different TPs previously used in the literature to drive protein import into the chloroplast stroma were fused to each Nif protein, namely: AtAROA ${ }_{\mathrm{TP}}$ (Klee et al., 1987), AtBCCP1 $1_{\mathrm{TP}}$, $A t \mathrm{CAB} 6_{\mathrm{TP}}, A t \mathrm{DNAJ} 8_{\mathrm{TP}}, A t \mathrm{GLTB} 2_{\mathrm{TP}}, A t \mathrm{TOCC}$ TP (Lee et al., 2008), $N t \mathrm{RBS}_{\mathrm{TP}}$ (Mazur and Chui, 1985), AtRBS1A Aт (Lee and Hwang, 2011), PsRBS2 AtRCA TP (Kim et al., 2010), NtSIRTP (Yonekura-Sakakibara et al., 1998) and synRBS (Engler et al., 2014) (details in Supplemental Table 7) and analysed for protein accumulation upon agroinfiltration of $N$. benthamiana leaves.

The use of TP sequences that extend beyond the peptidase cleavage site (complete TP, c) would produce recombinant proteins with additional amino acids left at the N-terminus after TP cleavage. Since this can affect protein functionality, shorter TP versions were generated by cloning only the TP coding sequence up until the cleavage recognition site predicted by ChloroP 1.1 server (Emanuelsson et al., 1999) (minimal TP, m). Constructs for expression of cytosolic Nif proteins (protein size control) and Nif fusions to complete and minimal versions for all 12 candidate TPs were generated (Figure 11A). 
(A)
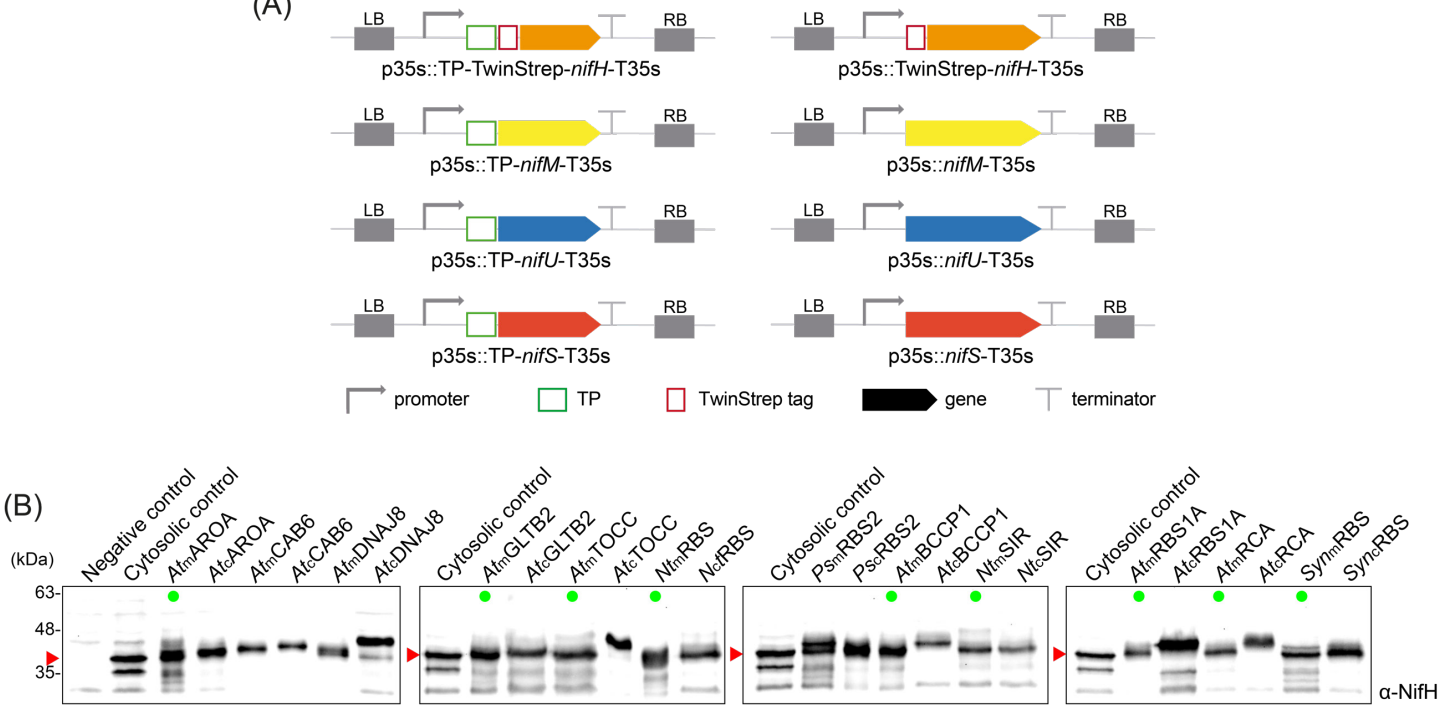

(C)

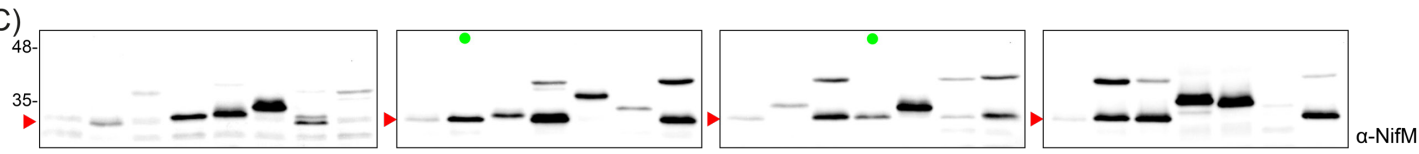

(D)

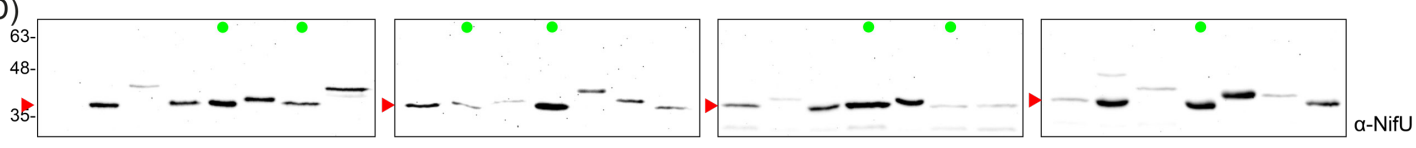

(E)

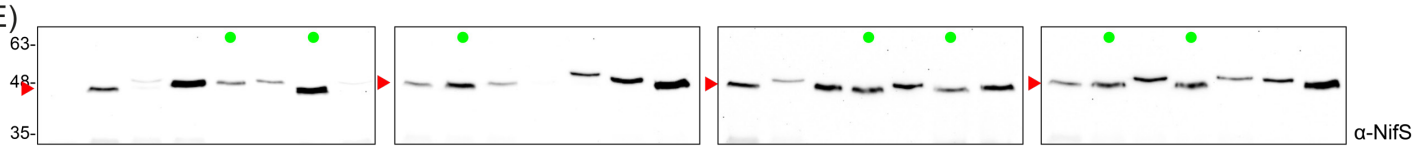

Figure 11. Screening of chloroplast transit peptides fused to NifH, NifM, NifU, and NifS proteins.

(A) Schematic representation of the constructs used in N. benthamiana-transient expression assays leading to TP-nif expression. (B-E) Western blot analysis of the product of expressing 12 TPs in full version (c, complete) and up until their predicted cleavage site by stromal peptidase (m, minimal) fused to the N-terminus of NifH (B), NifM (C), NifU (D), and NifS (E). Negative control refers to $N$. benthamiana leaves agroinfiltrated with Agrobacterium transformed with an empty vector. Cytosolic versions were expressed for Nif correct size control, expected sizes are Twin-Strep NifH: $35.3 \mathrm{kDa}$, NifM: $32.3 \mathrm{kDa}$, NifU: $34.4 \mathrm{KDa}$, and NifS: $44.3 \mathrm{kDa}$. For TP sizes, see Table S2. Green dots mark Nif proteins with the same electrophoretic mobility as their cytosolic controls, consistent with complete cleavage of the TP. The triangles in red mark the expected mature form of the protein.

Obtaining Nif proteins with the same electrophoretic mobility as the cytosolic versions (Figure 11B-E, green dots, and Supplemental Table 8) suggested correct import and cleavage of the TP, generating a chloroplast-recombinant Nif protein with none, or very few, residual amino acids in the N-terminus. Nif proteins with an increased size coinciding with that of the Nif protein plus the TP suggested neither import nor cleavage had taken place. The appearance of two bands could be indicative of partial import and cleavage (Figure 11B-E and Supplemental Table 8). 
Overall, the use of a minimal TP resulted in a higher motility band against the complete TP (Figure 11B-E). However, in cases such as AtmAROA ${ }_{\mathrm{TP}}, P s \mathrm{mRBS} 2_{\mathrm{TP}}, N t \mathrm{mRBS} \mathrm{TP}_{\mathrm{TP}}$ and SynmRBS ${ }_{\text {TP }}$ fusions, the minimal TP seemed to have lost its ability to import the Nif protein into the chloroplast as the band size coincided with that of the Nif protein plus the TP. It is noteworthy that only two minimal TPs, AtmGLTB2 $2_{\mathrm{TP}}$ and $A t \mathrm{mBCCP} 1_{\mathrm{TP}}$, performed properly for all Nif proteins.

(A)
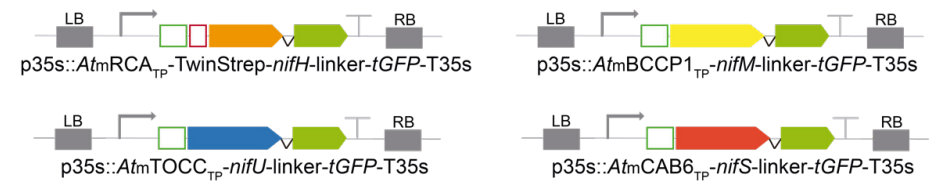

$\longrightarrow$ promoter $\square$ TP $\quad \square$ TwinStrep tag

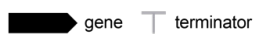

(B)
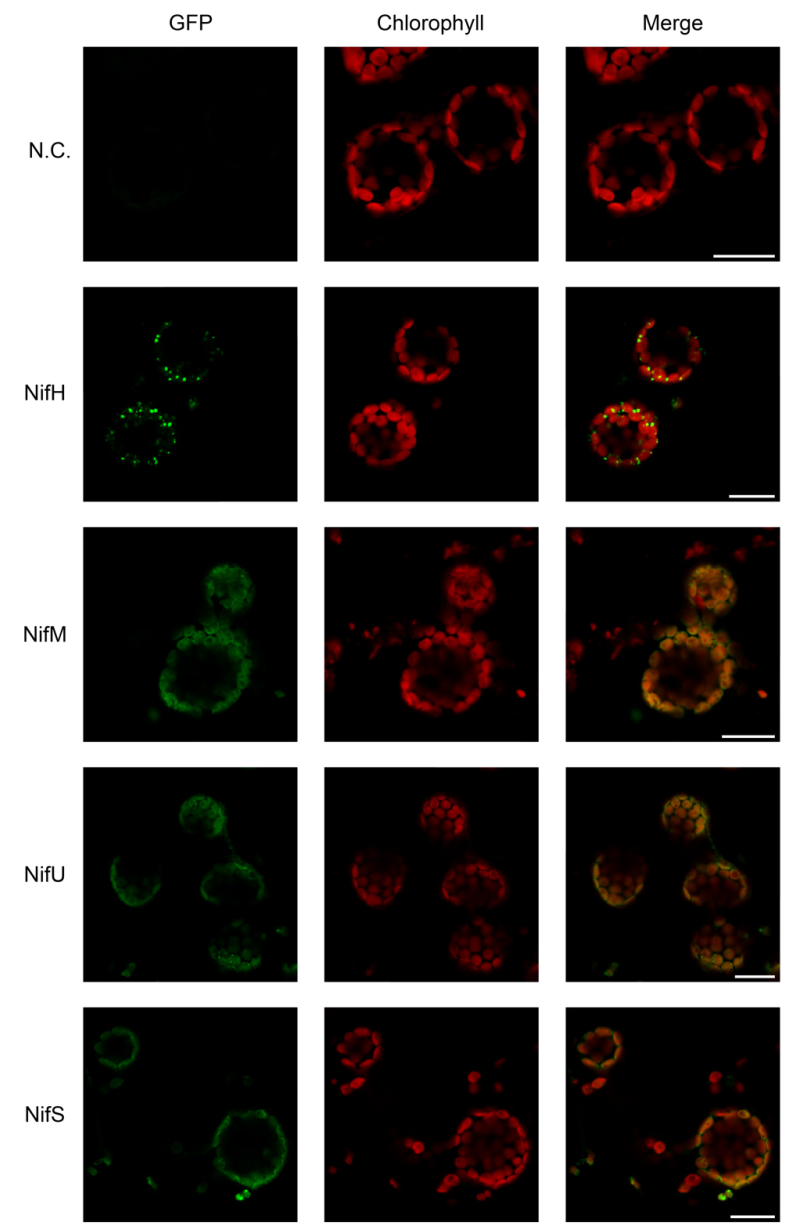

Figure 12. Confocal microscopy localization of stroma-targeted Nif proteins.

(A) Schematic representation of the constructs used in N. benthamiana-transient expression assays leading to Nif localization. (B) Confocal microscopy images of Nif proteins fused to tGFP. N.C. corresponds to 'negative control', a non-agroinfiltrated leaf. The three columns show the individual signals for tGFP (green, on the left) and chlorophyll autofluorescence (red, in the centre). On the right, the overlap of both signals (merge). 
One of the best-performing TPs for each specific Nif protein was selected (AtmRCATP for NifH, AtmBCCP1 $1_{\mathrm{TP}}$ for NifM, AtmTOCC ${ }_{\mathrm{TP}}$ for NifU, and AtmCAB6 ${ }_{\mathrm{TP}}$ for NifS), and Nif proteins were fused in their C-terminus to turbo GFP (tGFP) in order to visualize them within tobacco leaf mesophyll cells (Figures 12A and 13A). NifM, NifU, and NifS all showed a diffuse pattern of expression co-localized with chlorophyll, supporting correct targeting to the chloroplast stroma (Figure 12B). However, for NifH, we could observe tGFP concentrated in foci located within or around the chloroplasts, and that excluded chlorophyll. Chloroplast purification assays were performed in leaf tissue expressing Nif or Nif-tGFP fusion proteins. NifM, NifU, and NifS, as well as their corresponding tGFP fusions, were confirmed to accumulate mostly in the stroma soluble fraction, while the majority of NifH and NifH-tGFP appeared associated with membranes (Figure 13B). The similar behaviour of Nif and Nif-tGFP fusions validated the results obtained by microscopy, confirming that the fusion of the reporter protein did not lead to an artifactual localization and a clear indication of a NifH solubility problem. Antiisocitrate dehydrogenase (IDH) antibody was used to check for the presence of mitochondrial contamination in the isolation of chloroplasts (Figure 13B), and the negative results obtained suggest that the NifH-tGFP foci observed do not correspond to mitochondria, but rather, to chloroplast-associated bodies. 
(A)

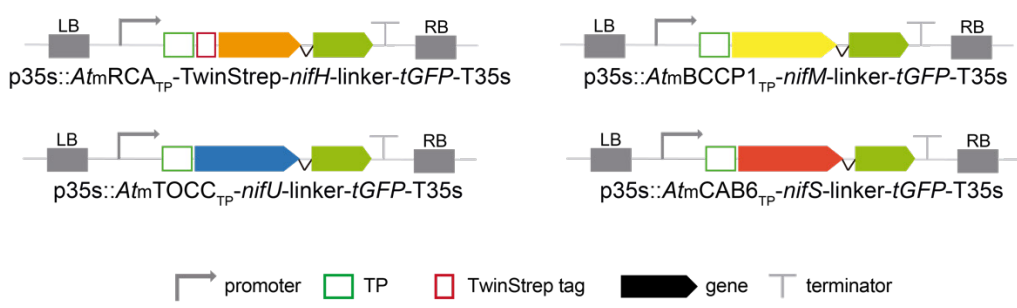

(B)
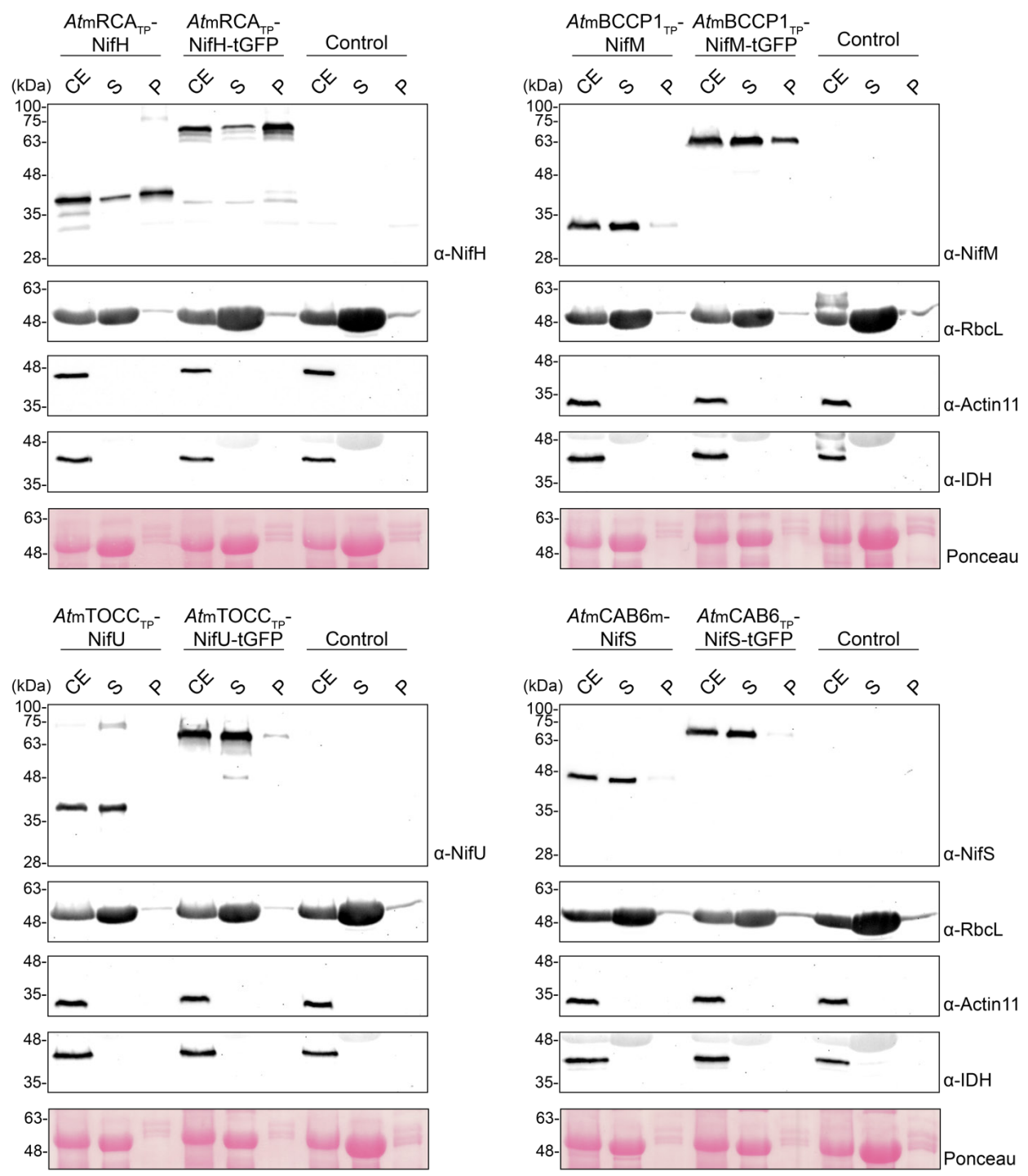

Figure 13. Subcellular fractionation confirming the localization of stroma-targeted Nif proteins.

(A) Schematic representation of the constructs used in $N$. benthamiana-transient expression assays leading to Nif localization. (B) Chloroplast purification assays. Total proteins extract before chloroplast purification $(\mathrm{CE})$. Intact chloroplasts were isolated and then broken to separate the soluble (S) and membrane-associated (P) fractions. Control refers to $N$. benthamiana plants agroinfiltrated with Agrobacterium transformed with an empty vector. Proteins were resolved by SDS-PAGE and detected by Western blot. Expected sizes are Twin-Strep NifH: $35.3 \mathrm{kDa}$, NifM: $32.3 \mathrm{kDa}$, NifU: $34.4 \mathrm{kDa}$, and NifS: $44.3 \mathrm{kDa}$ (27 kDa more in the case of tGFP fusions), RbcL: $52.7 \mathrm{kDa}$, Actin-11: $41.6 \mathrm{kDa}$, and IDH: $39 \mathrm{kDa}$. Note that the IDH blot was re-probed after striping anti-RuBisCO and some signal remains (upper band). 
To elucidate the nature and origin of these NifH-accumulating foci we co-expressed the

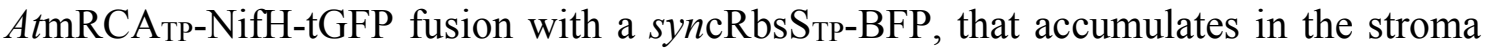
(Figure 14A). The co-localization of tGFP and BFP in a punctuate pattern (Figure 14B, arrows) supports that after NifH is imported into the chloroplast, it is included in vesicles together with other stroma soluble proteins, like BFP.

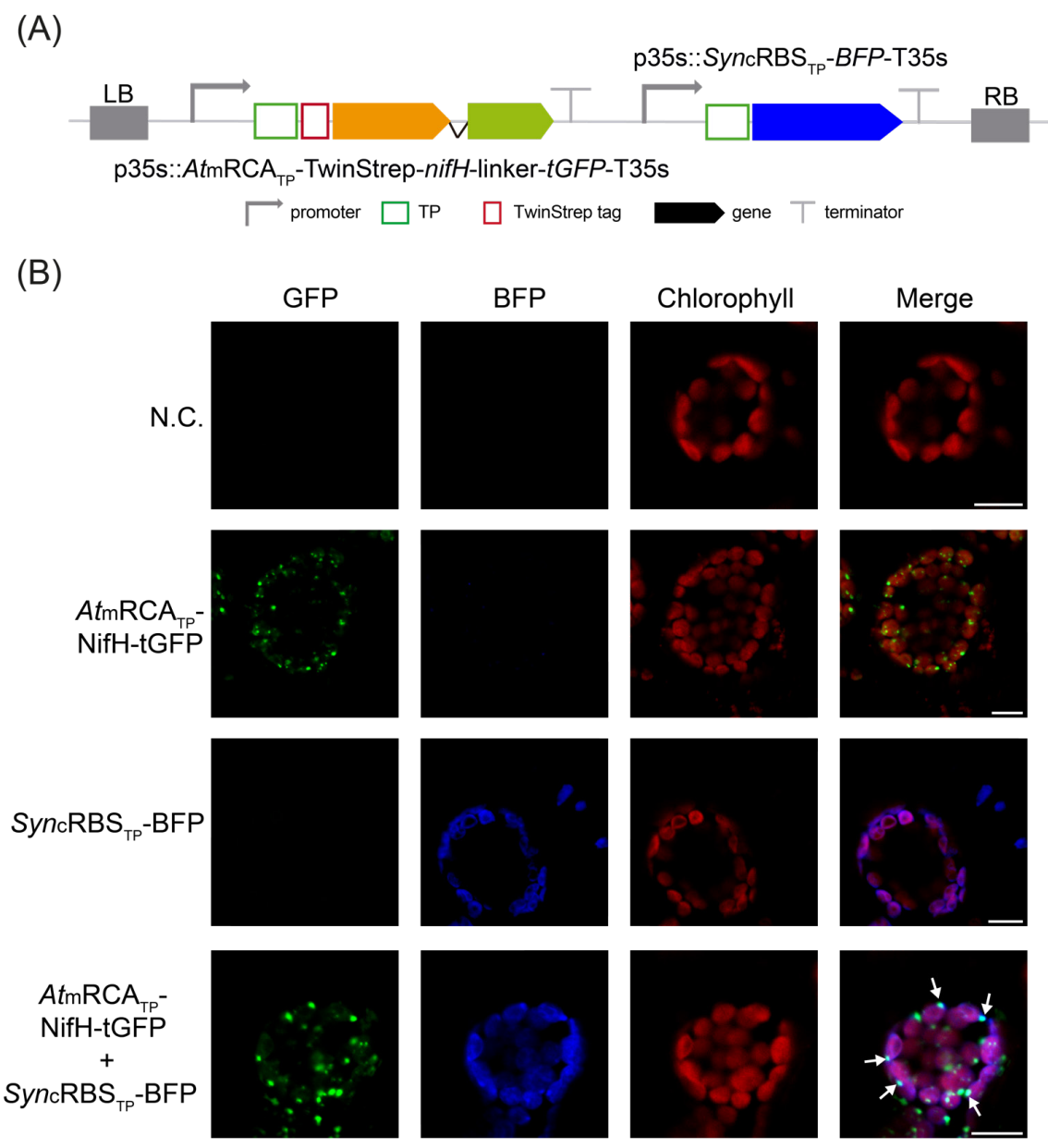

Figure 14. Expression of nifH alone leads to protein accumulation in foci originating from chloroplasts.

(A) Schematic representation of the constructs used in N. benthamiana-transient expression assays leading to NifH and stromal BFP co-localization. (B) Confocal microscopy images of NifH fused to tGFP and stromata targeted BFP. N.C. corresponds to 'negative control', a non-agroinfiltrated leaf. The four columns show the individual signals for tGFP (green), BFP (blue), and chlorophyll autofluorescence (red). On the right, the overlap of the three signals (merge). Note arrows pointing to the co-localization of green and blue signals in a punctuate pattern.

However, when both nifH and nifM genes were co-expressed, fused to $e G F P$ and $m$ Cherry, respectively (Figure 15A), NifH punctuated accumulation disappeared and could only be found diffuse within the chloroplast stroma (Figure 15B). This observation was corroborated by chloroplast purifications from cells co-expressing nifH, nifM, nifU, and nifS, where NifH could now be found mostly in the soluble protein fraction coming from the stroma (Figure 16A, C). All these data suggest that when expressed alone, NifH 
is accumulating in aggregates of immature protein that become included in vesicles, and that NifM is required for NifH to fold correctly and become soluble in the stroma.

(A)

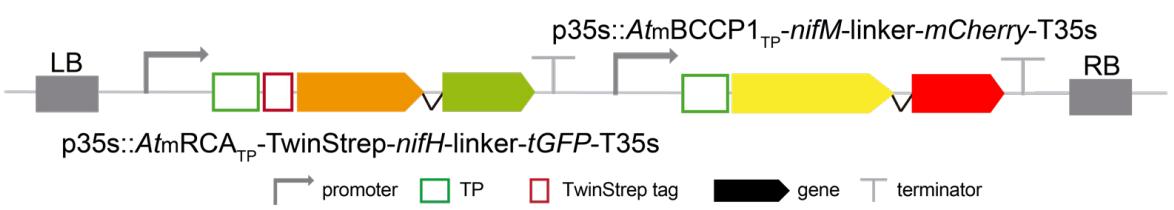

(B)
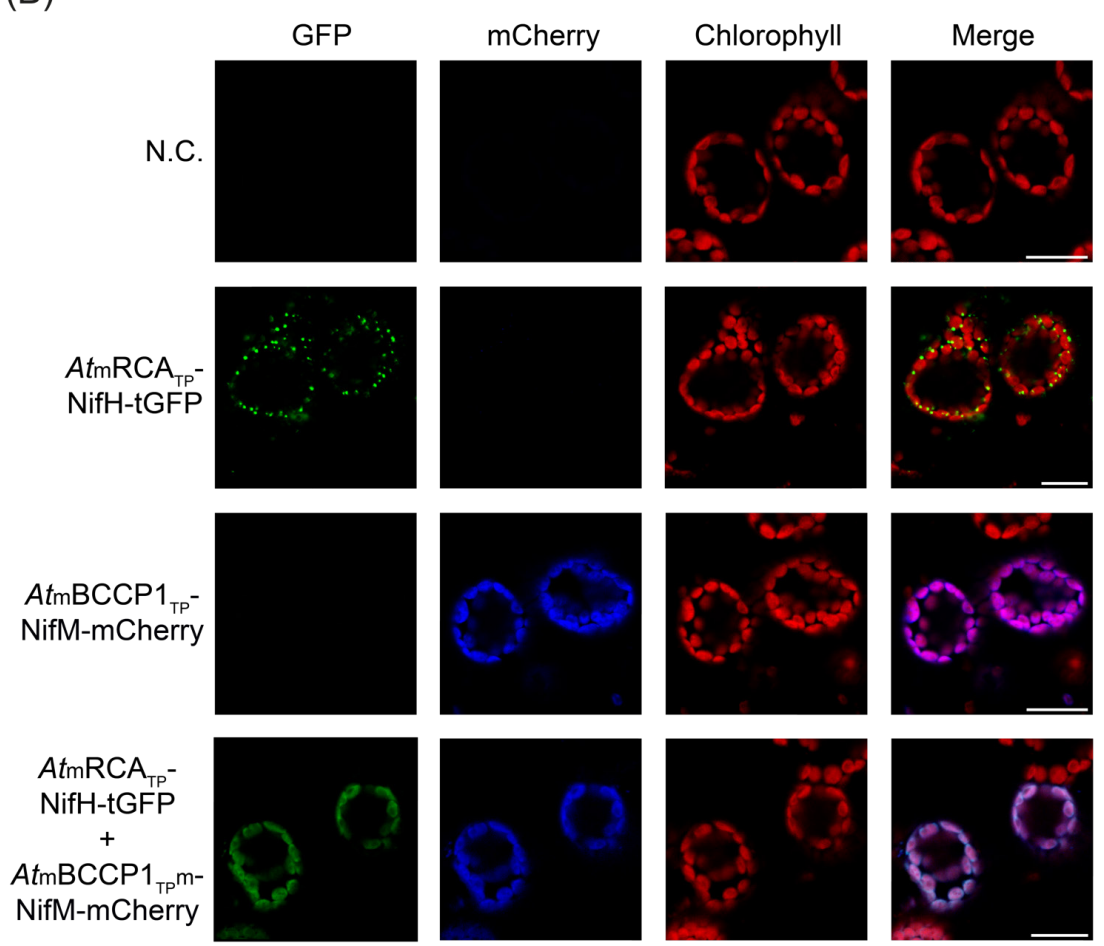

Figure 15. NifM is necessary for the accumulation of soluble NifH in the stroma.

(A) Schematic representation of the construct used in N. benthamiana-transient expression assays leading to NifH and NifM co-localization. (B) Confocal microscopy images of co-expressed NifH and NifM proteins fused to tGFP and mCherry respectively. N.C. corresponds to 'negative control', a nonagroinfiltrated leaf. The four columns show the individual signals for tGFP (green), mCherry (blue), and chlorophyll autofluorescence (red). On the right, the overlap of the three signals (merge). Note the colocalization of green and blue signals in a diffuse pattern within the chloroplast.

\subsubsection{Tobacco plants co-expressing nifH, nifM, nifU, and nifS exhibit Fe protein activity}

Once optimal conditions had been established for Nif protein accumulation and import to the chloroplast stroma, a multigenic construct was assembled with the tobacco synthetic nif genes and their selected TPs (Figure 16A). For protein purification, a single vector containing nifH, nifM, nifU, and nifS in addition to the silencing suppressor P19 was used. Following this approach in transient expression assays assures that all cells infected by Agrobacterium will get the complete gene set, and the purified Nif protein will have been expressed and maturated in cells containing simultaneously all of them. 
Tobacco leaves were collected at the end of the dark period of the third day after agroinfiltration when all four nif genes were expressed (Figure 16B) and Nif proteins accumulated in a soluble form within the chloroplast stroma (Figure 16C).

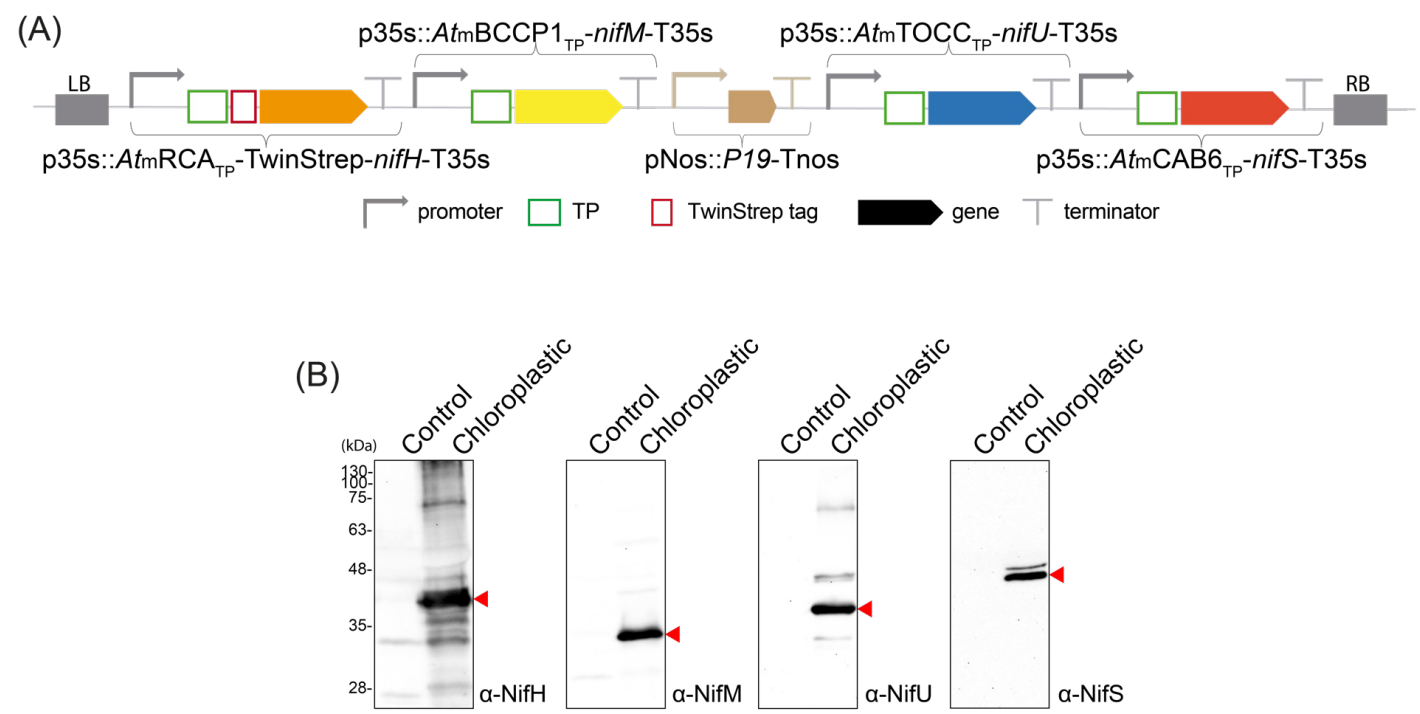

(C)
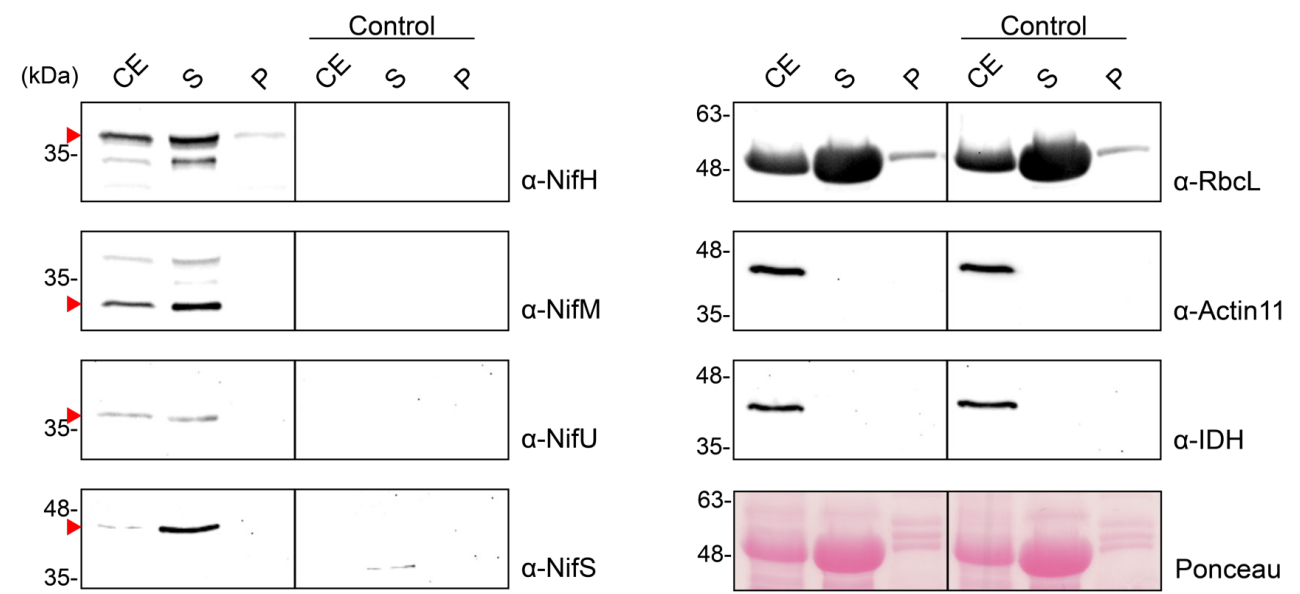

Figure 16. Co-expression of nifH, nifM, nifU, and nifS in chloroplasts of $N$. benthamiana

(A) Schematic representation of the multigenic construct used in N. benthamiana-transient expression assays leading to NifH, NifM, NifU, and NifS co-expression. (B) Western blot analysis of the agroinfiltrated tissue using antibodies targeting Strep-tagged NifH, NifM, NifU, and NifS as indicated. Control refers to $N$. benthamiana leaves agroinfiltrated with Agrobacterium transformed with an empty vector. Expected sizes are Twin-Strep NifH: $35.3 \mathrm{kDa}$, NifM: $32.3 \mathrm{kDa}$, NifU: $34.4 \mathrm{kDa}$, and NifS: 44.3 $\mathrm{kDa}$. (C) Chloroplast purification assays. Total proteins extract before chloroplast purification (CE). Intact chloroplasts were isolated and then broken to separate the soluble (S) and membrane-associated (P) fractions. Control refers to $N$. benthamiana plants agroinfiltrated with Agrobacterium transformed with an empty vector. Proteins were resolved by SDS-PAGE and detected by Western blot. Expected sizes are RbcL: $52.7 \mathrm{kDa}$; Actin-11: $41.6 \mathrm{kDa}$ and IDH: $39 \mathrm{kDa}$. The triangles in red mark the mature form of the protein.

NifH from $N$. benthamiana chloroplasts was purified using Twin-Strep tag for affinity chromatography (Figure 17A-D). Various bands could be detected in the elution fraction after NifH purification. Peptide mass fingerprinting identified them as a RuBisCo subunit 
(probably a contaminant), the maturase NifM, and NifH degradation products or multimers (Supplemental Figure 1). NifH migrated as a close double band in SDS-PAGE (Figure 17C-D); however, it was not due to N-terminal degradation or unspecific cleavage of the TP by the stromal peptidase, since Edman degradation of the purified protein confirmed a unique N-terminal sequence starting at the predicted location for TP cleavage (Supplemental Figure 2). NifH doublets not related to post-translational modifications have been previously observed both in diazotrophic bacteria and in heterologous hosts (Howard et al., 1986; Roberts et al., 1978). Detection after Western blots using anti-NifM showed a band that corroborated some degree of co-elution due to interaction with $\mathrm{NifH}$. The same was observed using an anti-NifU antibody (Supplemental Figure 3), suggesting the on-going maturation of a subpopulation of the Fe protein in the samples.

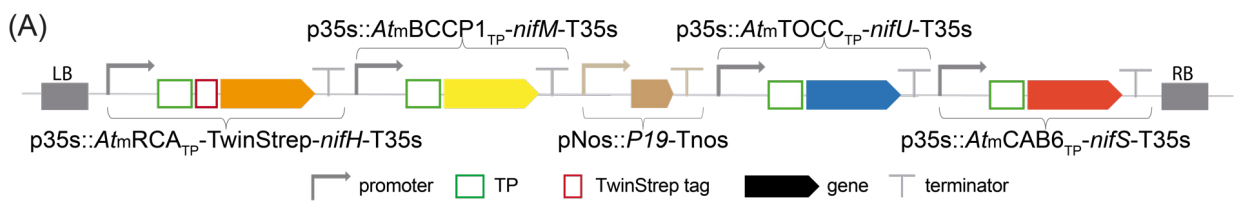

(B)
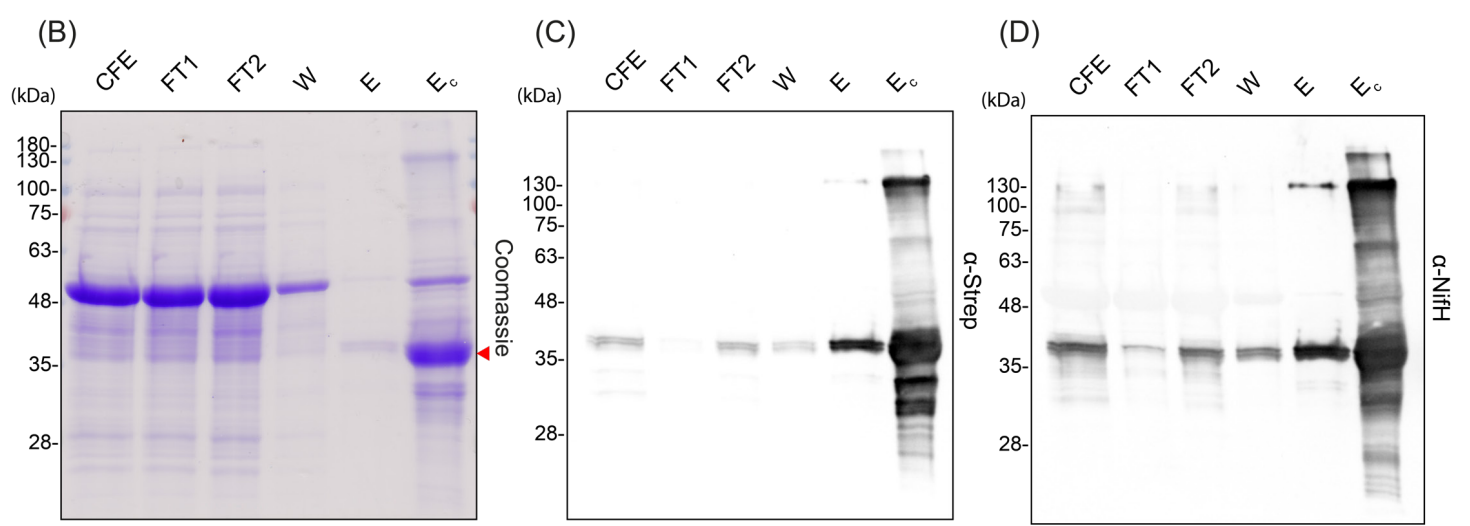

Figure 17. Purification of NifH from chloroplasts of $N$. benthamiana co-expressing nifH, nifM, nif $U$, and nifS.

(A) Schematic representation of the multigenic construct used in N. benthamiana-transient expression assays leading to NifH purification. (B) Coomassie staining illustrating the Strep-tactin purification procedure of $A$. vinelandii $\mathrm{NifH}$ expressed in leaves of $N$. benthamiana. CFE, cell-free extract (soluble fraction following centrifugation and filtering of disrupted leaf tissue); FT, Strep-Tactin flow-through fraction; W, wash fraction; E, biotin-eluted fraction; Ec, concentrated eluate. (C-D) Western blot analysis of the Strep-Tactin purification procedure. Antibodies targeting Strep-tag (C) or NifH (D) were used for visualization of the purified NifH protein.

Pure NifH isolated from chloroplasts exhibited a light yellowish/brownish colour, characteristic of Fe-S proteins. Its activity was determined by using in vitro acetylene reduction assays in which the $N$. benthamiana $\mathrm{NifH}$ was incubated with MoFe protein purified from $A$. vinelandii. N. benthamiana Fe protein activity varied among preparations, supporting a maximum specific activity of $189 \mathrm{nmol}$ of ethylene formed per 
minute and milligram of MoFe protein at a $N$. benthamiana Fe protein $/ A$. vinelandii $\mathrm{MoFe}$ protein molar ratio of 25 (Table 1).

Table 1. Maximum nitrogenase MoFe protein activity supported by $N$. benthamiana $\mathrm{NifH}$ and $\boldsymbol{A}$. vinelandii $\mathbf{N i f H}$. Highest result obtained in three positive independent biological experiments. *Defined as nmol of $\mathrm{C}_{2} \mathrm{H}_{4}$ formed per minute and milligram of MoFe protein (mean \pm s.d., $\mathrm{n}=2$ technical replicates).

\begin{tabular}{c|cc} 
Source of Fe protein & $\begin{array}{c}\text { Acetylene reduction } \\
\text { assay * }\end{array}$ & $\begin{array}{c}\text { Fe protein to MoFe } \\
\text { protein molar ratio }\end{array}$ \\
\hline N. benthamiana NifH & $189 \pm 5$ & 25 \\
A vinenlandii NifH & $1194 \pm 65$ & 25
\end{tabular}

Although the activities detected are low compared to those of $A$. vinelandii NifH (a maximum of $16 \%$ activity was obtained in chloroplast NifH compared to $A$. vinelandii $\mathrm{NifH}$ ), these results indisputably show that tobacco chloroplasts are capable of accumulating functional $\mathrm{Fe}$ protein, at least when its maturation and $[\mathrm{Fe}-\mathrm{S}]$ cluster assembly is separated in time from the process of photosynthesis (activity is shown for samples collected at the end of the dark period). Future efforts will be directed at designing strategies to protect $\mathrm{NifH}[\mathrm{Fe}-\mathrm{S}]$ clusters from oxygen as a means to increase its activity, and to test the effect of photosynthesis during the light period on the protein metal content and activity. The influence of the Twin-Strep-tag cannot be considered as a factor accounting for the lower chloroplast NifH activity, since purified $A$. vinelandii Strep-tagged NifH showed almost the same activity as the non-tagged version (Eseverri et al., 2020).

In the chloroplast, other pathways exist for Fe-S protein assembly, like the sulfur mobilization pathway (SUF), raising the question of the requirement for NifU and NifS. To test their role in the assembly and delivery of [Fe-S] clusters to NifH within the chloroplast, we performed the same experiments in $N$. benthamiana leaves expressing only nifH and nifM (Figure 18A). NifH from $N$. benthamiana chloroplasts was successfully purified using Twin-Strep tag for affinity chromatography (Figure 18B-D). However, in the absence of NifU and NifS, no Fe protein activity could be detected, suggesting that the chloroplast assembly and transfer factors are not able to provide enough $[\mathrm{Fe}-\mathrm{S}]$ clusters to result in functional Fe protein. These results open a feasible avenue of work on plastid engineering for the generation of nitrogen-fixing plants. 


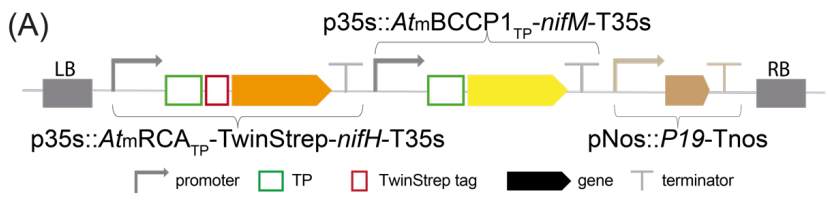

(B)

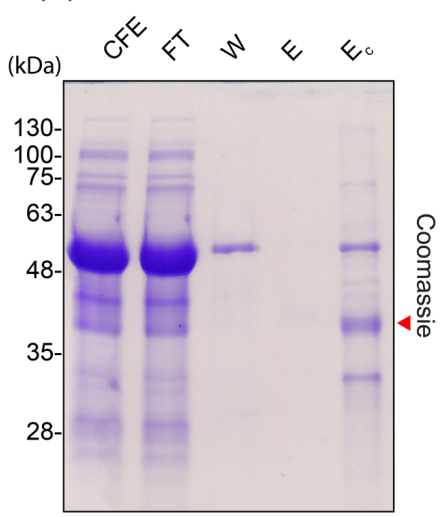

(C)

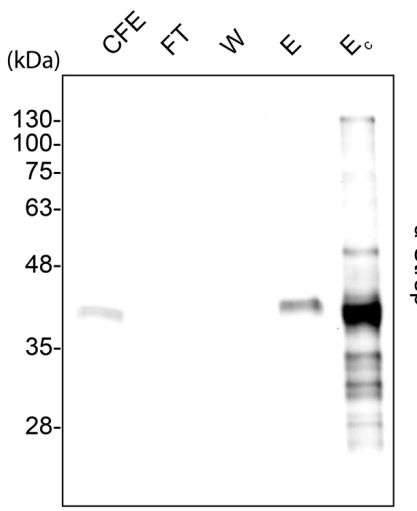

(D)

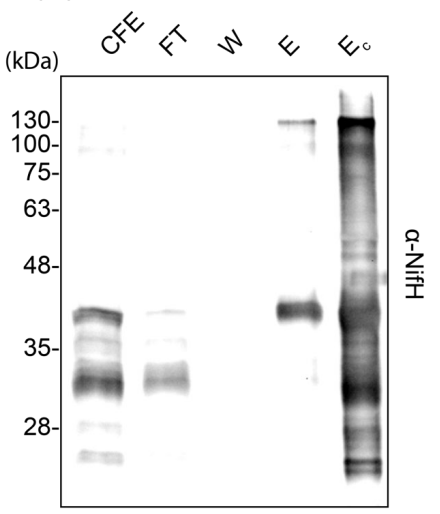

Figure 18. Purification of NifH from chloroplasts of $N$. benthamiana co-expressing nifH and nifM. (A) Schematic representation of the multigenic construct used in $N$. benthamiana transient expression assays leading to NifH purification. (B) Coomassie staining illustrating the Strep-tactin purification procedure of $A$. vinelandii $\mathrm{NifH}$ expressed in leaves of $N$. benthamiana. CFE, cell-free extract (soluble fraction following centrifugation and filtering of disrupted leaf tissue); FT, Strep-Tactin flow-through fraction; W, wash fraction; E, biotin-eluted fraction; Ec, concentrated eluate. (C) Western blot analysis of the Strep-tactin purification procedure using antibodies targeting Strep-tag. The expected sizes are Twin-Strep NifH: $35.3 \mathrm{kDa}$. (D) Western blot analysis of the Strep-tactin purification procedure using antibodies targeting NifH.

\subsection{Discussion}

\subsubsection{Optimization of nif gene synthetic design and chloroplast targeting}

The use of nif genes synthetically designed to maximize expression in tobacco led to much higher protein accumulation than the use of the yeast-optimized versions (Figure $9)$, although the CAIs for these synthetically designed genes $(0.76,0.77,0.79$ and 0.72 for nifH, nifM, nifU, and nifS, respectively) were in all cases slightly lower than those of the ones optimized for yeast (Supplemental Table 6). Adapting the codon usage frequencies of a foreign sequence to that of the host organism was not enough to guarantee high gene expression and protein accumulation, and the advantage obtained by the inclusion of more parameters in the design of the synthetic genes clearly outweighed the penalty of a small decrease in the CAI. More complex approaches, like the one used here, should be considered when the goal is to achieve high levels of recombinant protein. Note that using a complete synthetic design allowed us to obtain NifH protein levels 170 times higher than those achieved by using simply a codon-optimized sequence (Figure 9D), amounts that were enough for Fe protein purification and activity tests. Although no significant Nif degradation products could be detected linked to the light period (Figure 
10B), oxygen damage could still affect Nif proteins in the form of cluster loss, which remains to be studied.

The use of the nuclear transformation approach requires that the protein import into the plastid must be optimized as well. The use of TPs is a strategy that has already been used in many biotechnological applications to direct recombinant proteins to the chloroplast stroma (Van den Broeck et al., 1985; Hoppmann et al., 2002; Kavanagh et al., 1988; Kim et al., 2009, 2010; Lee et al., 2008; Zhong et al., 2003). However, we found that most of the TPs described in the literature included a sequence longer than the transit peptide, leaving scar amino acid residues in the N-terminus of the imported proteins after TP cleavage. We used bioinformatics tools to predict the minimal sequence required to direct recombinant proteins to the subcellular compartment and that would leave the minimal scar after cleavage (ChloroP 1.1 server (Emanuelsson et al., 1999)). In most cases, the extended version of the TP did not work better than the minimal version, thus having a longer sequence does not seem beneficial, and only the minimal versions should be used in the future, as they comprise cleaner and as efficient tools for recombinant protein targeting (Figure 11).

As expected, TPs showed different behaviour when fused to different Nif proteins, highlighting the influence of cargo proteins in import efficiency (Shen et al., 2017). Although some TPs, like the one from the biotin carboxyl carrier protein $\left(A t \mathrm{mBCCP} 1_{\mathrm{TP}}\right)$ and ferredoxin dependent glutamate synthase (AtmGLTB2 $\left.2_{\mathrm{TP}}\right)$, seemed to perform well in all four cases (Figure 11), diversifying the TPs used is desirable for synthetic biology efforts in which multiple nif genes are to be co-expressed. In this case, avoiding the repetition of TPs would be beneficial since homologous recombination between repeated sequences is a major cause of genomic rearrangements, producing insertion/deletions and translocations.

Targeting of proteins to mitochondria and chloroplasts is generally highly specific, but an increasing number of proteins imported dually into both organelles have been described (Peeters and Small, 2001). In this work, chloroplast purification assays clearly show that the selected TPs drive Nif proteins primarily to the chloroplast, and microscopy of the tGFP fusions confirms specific organelle targeting.

The observation of cleavage of the transit peptide in a Western blot is often indicative of correct localization of the recombinant protein but should be confirmed. Our results indicate that when expressed alone, NifH is imported into the organelle and the TP is correctly processed. However, NifM co-expression was necessary to lead to soluble stroma NifH accumulation. With a few exceptions, the production of an active $\mathrm{NifH}$ is 
known to depend on NifM (Howard et al., 1986), and our data suggest that this requirement extends to plant cells.

The observed compartmentalization of NifH in the absence of NifM is reminiscent of the bodies formed in plant cells during piecemeal chlorophagy, a process that involves the formation of small vesicle-like structures that bud from plastids and are delivered to the vacuole for degradation (Otegui, 2018). All the gathered information suggests improper folding of the NifH protein when expressed alone, which triggers this or another similar degradation pathway within the plant cell.

\subsubsection{Purification of active $\mathrm{NifH}$ from tobacco chloroplasts}

The recovery of relatively high Fe protein activity expressed in a plant is a major accomplishment. The activity obtained is still lower than that of endogenous $A$. vinelandii $\mathrm{NifH}$ or the one obtained from yeast mitochondria (López-Torrejón et al., 2016), so future efforts should be directed at fine-tuning its maturation and/or preventing damage of its [4Fe-4S] cluster by oxygen. As NifH is extremely sensitive and irreversibly damaged by oxygen (Shah et al., 1973), finding its functional form within the chloroplast indicates this organelle is able to provide an anoxygenic (enough) environment for the enzyme, at least during the night period, after which tissue collection took place. This is in accordance with the observation that $C$. reinhardtii mutants in chlL, an essential chloroplast gene required for chlorophyll biosynthesis in the dark, could be partially complemented with the K. pneumoniae nifH (Cheng et al., 2005).

The co-elution of NifM with NifH could be interpreted in the light of NifM-mediated maturation of NifH (Supplemental Figure 3A). It is possible that in our transient expression system the time in which the two proteins cohabitate within the chloroplast is too short for complete maturation of $\mathrm{NifH}$ and in the future, it will be important to test whether, in stable transformants, this interaction diminishes leading to a higher activity of (properly matured) NifH. The co-elution of NifU with NifH points to an interaction between these two proteins, suggestive of an actual cluster transfer occurring between the two (Supplemental Figure 3B). In fact, $A$. vinelandii NifU isolated from $N$. benthamiana chloroplasts was found to be functional in transferring [4Fe-4S] clusters to apo-NifH in in vitro assays (Eseverri et al., 2020).

Similarly, recent experiments co-expressing NifU and NifS targeted to chloroplast showed a fraction of NifS co-purifying with NifU, which carried a permanent [2Fe-2S] cluster per dimer (Eseverri et al., 2020). However, when NifU was expressed alone, its 
Fe content was much lower, suggesting that NifS was also active in transferring the sulfur necessary for the assembly of [Fe-S] clusters in the chloroplast.

The lack of NifH activity when it was only co-expressed with nifM, but not nifU or nifS, suggests that the chloroplast machinery cannot efficiently transfer [4Fe-4S] clusters to NifH. We cannot completely exclude, however, the possibility that the effect of NifU and NifS is indirect and being asserted by other Fe-S protein that in turn has a positive effect on NifH, or by reducing the possible stress caused by excessive free iron. Previously, only a very low and difficult to quantify NifH activity had been detected in transplastomic plants expressing NifH and NifM after incubation of whole plants under a low oxygen atmosphere (Ivleva et al., 2016). By using a different technical approach and incorporating NifU and NifS, we have obtained significant levels of activity for NifH in plants grown with no manipulation of the atmospheric oxygen content, demonstrating that the nuclear-encoding of plastid-targeted nif genes constitutes a worthy strategy for engineering nitrogenase assembly in the plastids of higher plants. 


\section{Optimization of nitrogenase Fe protein biosynthesis in rice: Arabidopsis transit peptides as tools for trait engineering in rice plastids ${ }^{\ddagger}$}

\subsection{Introduction}

Plastids are organelles found within plants and algae cells. They are enclosed by two membranes, the outer and inner envelope membranes, that separate the cytosol from the stroma. Phylogenetic analyses show that plastids originated from the endosymbiosis of a cyanobacterial ancestor (Yoon et al., 2004). In higher plants, all plastids derive from undifferentiated pro-plastids that develop into different morphological and functional types in a developmental and tissue-specific manner and which have distinct functions mediated by different proteomes (Daher et al., 2010; Kleffmann et al., 2004; Siddique et al., 2006). Among them are chloroplasts, housing green chlorophyll pigments that mediate photosynthesis; leucoplasts, colourless plastids found in non-photosynthetic tissues which include starch-storing amyloplasts and lipid-storing elaioplast; and chromoplasts, responsible for the carotenoid pigments found in flowers and fruits (Wise, 2007). Plastids carry out a diverse set of metabolic functions, both in assimilation carbon, sulfur, and nitrogen, and in the production of lipids, amino acids, plant hormones, and many secondary metabolites (Waters and Pyke, 2005).

Plastid genomes of land plants have suffered a huge reduction by transferring genes to the nuclear genome (Kleine et al., 2009). More than 95\% of chloroplast proteins are nucleus-encoded and synthesized on cytosolic ribosomes (Shi and Theg, 2010), which account for around 2,100 in A. thaliana and up to 4,800 proteins in O. sativa (Richly and Leister, 2004). Such transfer underscores the importance of differentially expressing nuclear genes encoding plastid proteins according to tissue and developmental stage (Kleffmann et al., 2004) and the regulation of the import of proteins from the cytosol (Chu et al., 2020; Chu and Li, 2018; Jarvis and López-Juez, 2013), as these processes ultimately determine plastid biogenesis and plant development.

Nuclear-encoded plastid-targeted proteins are synthesized as precursors containing an Nterminal targeting sequence called the transit peptide (Chua and Schmidt, 1978; Dobberstein et al., 1977; Kleffmann et al., 2004). TPs bind the targeting receptors

\footnotetext{
\$ This section has been partially published in Frontiers in Plant Science:

Eseverri, Á., Baysal, C., Medina, V., Capell, T., Christou, P., Rubio, L.M., and Caro, E. (2020) Transit Peptides From Photosynthesis-Related Proteins Mediate Import of a Marker Protein Into Different Plastid Types and Within Different Species. Front. Plant Sci., 11, 1. doi: $10.3389 /$ fpls.2020.560701
} 
associated with the translocons at the outer/inner envelope membrane of plastids (TOC and TIC, respectively) and direct the import of precursor proteins across the organellar double membrane (reviewed in Chotewutmontri et al., 2017). After precursor translocation into the stroma, the TP is cleaved off by the Stromal Processing Peptidase (SPP), and the mature protein folds into its native conformation and stays within the stroma or continues its journey to the thylakoids (Richter and Lamppa, 2002).

TPs are necessary and sufficient for protein import into plastids; the removal of the gene part coding for it renders a protein that remains in the cytosol, while the addition of the TP to a non-plastid protein can direct it to the organelle (Bruce, 2001). The length of TPs is very heterogeneous, ranging from 20 to 150 amino acids, depending on the position of the processing site by the SPP (Balsera et al., 2009). TP primary sequence alignments have been used to identify conserved motifs responsible for specific import, but their conservation, amino acid composition, and organization are very reduced (Bruce, 2000).

Contrarily, the general import pathway seems to be very conserved, as evidenced by the fact that most TOC/TIC components are maintained in all land plant species (Tello-Ruiz et al., 2016). Their expression is highly regulated. In A. thaliana, the receptors for the initial pre-protein binding, atToc59 and atToc33 are highly expressed in green tissues, while atToc132/120 and atToc34 are uniformly expressed across all tissues (reviewed in $\mathrm{Chu}$ and $\mathrm{Li}, 2018$ ). Arabidopsis and rice TIC/TOC systems are very conserved in terms of members of the complex and their specific expression patterns (Supplemental Figure $4)$.

Recent data suggest that the availability of TOC isoforms that bind with different specificity to each transit peptide can determine the import of precursor proteins (Chu et al., 2020; Chu and Li, 2018; Dutta et al., 2014; Jarvis and López-Juez, 2013). In vitro binding assays showed that atToc159 binds preferentially to transit peptides of photosynthetic proteins and atToc132 binds transit peptides of non-photosynthetic proteins (Inoue et al., 2010; Ivanova et al., 2004; Smith et al., 2004). Swapping of TPs among proteins confirmed their role in the determination of plastid-type import selectivity (Wan et al., 1996; Yan et al., 2006). The precursor-specificity of the pathway seems to be restricted to the TOC, since TIC components were found to associate equally with all kinds of plastid imported proteins (Chen et al., 2002; Jarvis, 2008; Kovacheva et al., 2005).

Detailed characterization of different TPs, and their specific plastid-type import preference, is of the utmost importance to engineer useful traits determined by nuclearencoded recombinant plastid proteins, as it is the case in the engineering of $\mathrm{NifH}$ 
biosynthetic pathway in rice plastids. Here we show that the TPs of four Arabidopsis proteins related to photosynthetic, photo-protection processes, and fatty acid biosynthesis direct highly efficient import of a recombinant reporter protein to chloroplasts and leucoplasts. These TPs also show 100\% import efficiency in rice callus and the shoot and root tissues of rice plants, suggesting that translocon components mediating import of the reporter protein are distributed equally in these tissues or that the TPs bind promiscuously to predominant TOC/TIC isoforms.

Further work on the optimization of NifH biosynthetic pathway in rice plastids consisted in the improvement of Nif protein production and accumulation. Protoplast transient expression assays showed the importance of codon usage and GC content criteria in gene optimisation, and the requirement of NifM for NifH soluble protein accumulation in the stroma of rice cells. Although our efforts to generate nif-expressing stable rice lines had a negative outcome, further efforts should consider these findings in the design phase of further iterations of the DBTL cycle.

\subsection{Results}

\subsection{1. nif gene synthetic design for rice}

In an attempt to translate the knowledge gained from the experiments in tobacco to the engineering of nitrogenase Fe protein biosynthesis in the staple crop rice, we decided to start by focusing our efforts on maximizing nif gene expression within the context of rice cells. For that, we tried two different approaches. On the one hand, we ordered the synthesis of rice codon-optimized sequences for $A$. vinelandii nifH, nifM, nifU, and nifS to GenScript (Piscataway), which, using their proprietary algorithm, designed versions with CAI values of $0.90,0.89,0.87$ and 0.87 , respectively (Biologics International Corp codon adaptation index calculator and rare codon analyser).

On the other hand, in an effort to enhance nif gene optimization specifically for rice, we analyzed the nifH, nifM, nifU, and nifS sequences provided by GenScript in search of mRNA instability motifs, monocotyledonous plant polyadenylation signals, and AT strings (Jackson et al., 2014; Ji et al., 2007; Shen et al., 2008) (Supplemental Table 9), and partially removed them by PCR (Supplemental Table 10). This approach allowed us to test the influence of these undesired motifs in the accumulation of Nif proteins. As part of our previous work in nitrogenase engineering, we also had available the endogenous A. vinelandii nifH, nifM, nif $U$, and nifS gene sequences, yeast codon-optimized sequences (as used in López-Torrejón et al., 2016) and tobacco synthetic designs (as used in Section 7). 
Therefore, we compared protein accumulation in rice derived from those five different nif gene variants. We generated multigenic constructs comprised of a transcriptional unit where the pZmUbil promoter directed the expression of each nif variant and a second transcriptional unit where the same promoter directed the expression of eGFP. Transient expression assays in O. sativa protoplasts isolated from sheath and stem tissues were performed to assess the fitness of each of the designs (Figures 19A and 20A). Nif protein accumulation (Figure 19B) was compared using the level of eGFP to normalize for differences in overall recombinant protein expression and relativized to the value of the A. vinelandii endogenous sequences (Figure 19C).

The results showed a consistent pattern in both NifH and NifM accumulation, with the yeast codon-optimized sequences ranking as the worst-performing ones, followed by the synthetic designs for tobacco (Figure 19D). The rice codon-optimizations had a similar performance to the original $A$. vinelandii sequences, only overcome by the synthetic design for rice, in the case of NifM. This result partially supported our theory that the removal of deleterious motifs can be beneficial for the expression and accumulation of Nif proteins (Supplemental Table 11).

The sequences of nifH and nifM, optimized for their expression in yeast (López-Torrejón et al., 2016) and tobacco (Section 7.2.1) showed a theoretical detrimental optimization for expression in rice due to codon usage, with CAIs of 0.57 and 0.60 in the case of yeast optimization, and 0.53 and 0.56 in the case of the tobacco synthetic design, respectively (Supplemental Table 11). Conversely, the CAIs of nifH and nifM originally from $A$. vinelandii (0.83 and 0.85$)$, the rice codon-optimization (0.90 and 0.89$)$, and the rice synthetic design (0.89 and 0.89 ), already pointed to a successful heterologous gene expression in rice and matched with the NifH and NifM protein accumulation actual patterns observed experimentally. Interestingly, for latter three versions of nifH and nifM direct correlation between GC and GC3 content values and higher protein accumulation was observed (Supplemental Table 11). 
(A)

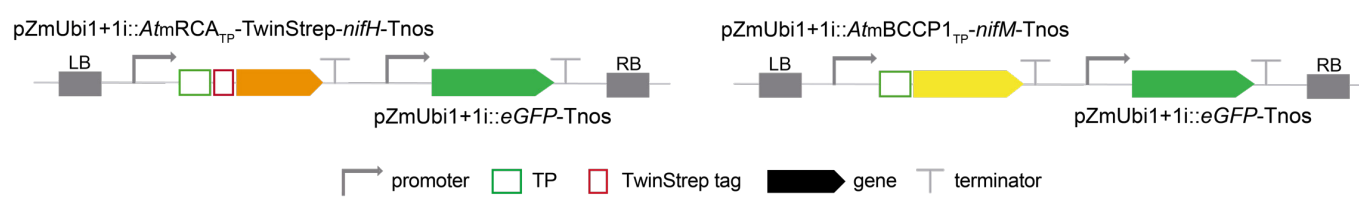

(B)

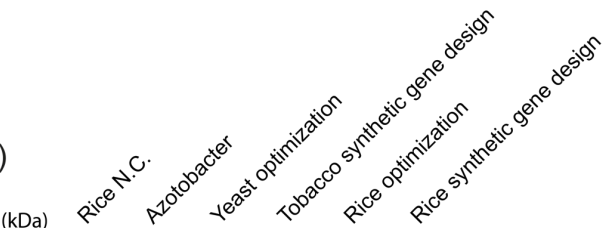

(C)
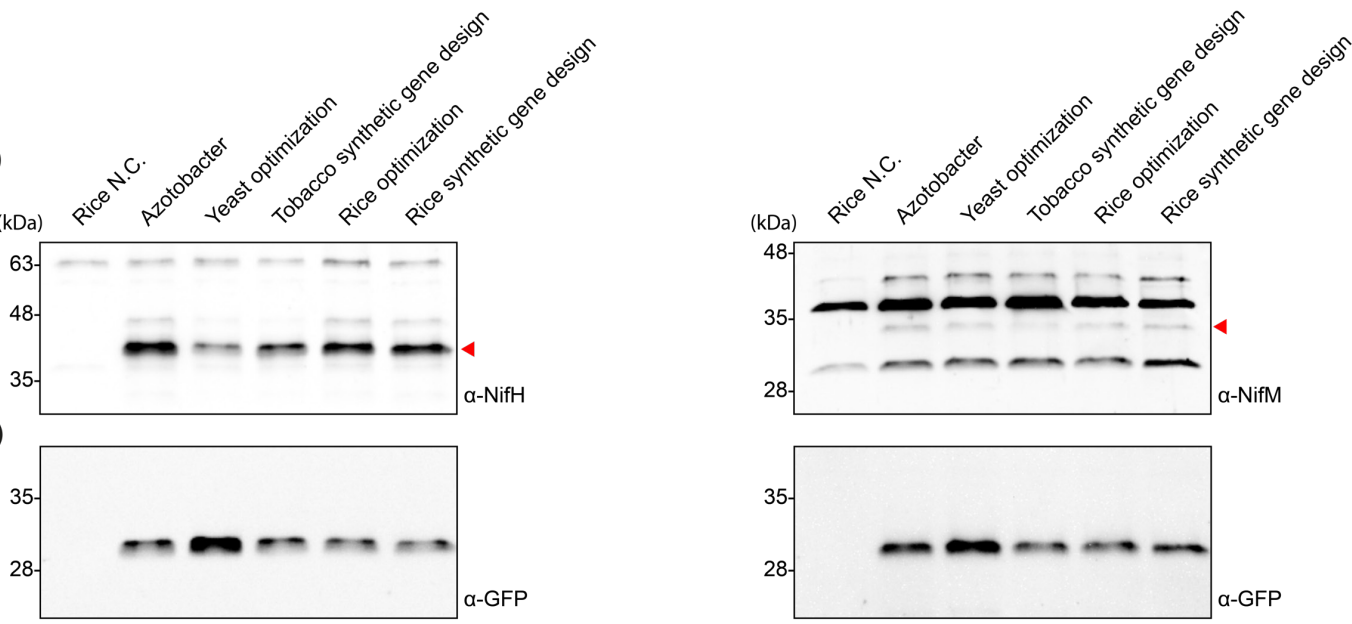

NifH

(D)
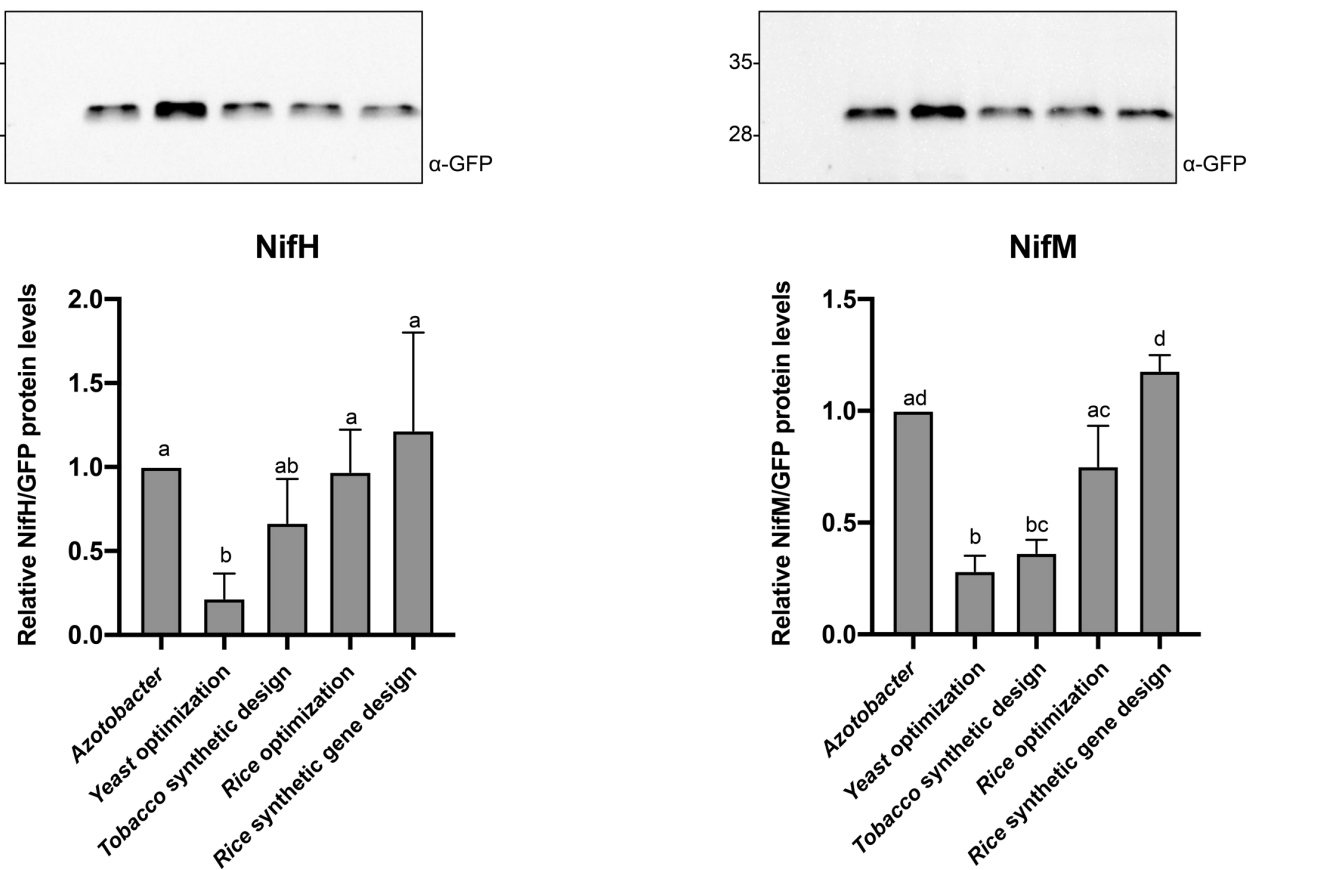

NifM

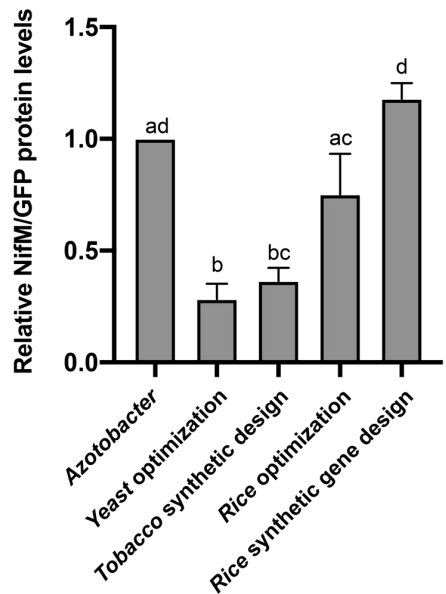

Figure 19. Effect of using diverse nifH and nifM gene optimizations in rice.

(A) Schematic representation of the constructs used in $O$. sativa-transient expression assays leading to nifH or nif $M$ gene and eGFP simultaneous expression. (B) Western blot analysis of protoplasts isolated from rice accumulating NifH (left) and NifM (right) when expressed from its native $A$. vinelandii gene, a yeast codon-optimized sequence, a synthetically designed gene optimized for expression in tobacco, a rice codon-optimized sequence, or a synthetically designed gene optimized for expression in rice. N.C. refers to non-transformed protoplasts. The triangles in red mark the expected mature form of the protein. Intense 30 and $35 \mathrm{kDa}$ non-specific bands can be observed when using $\alpha$-NifM antibodies. Expected sizes are Twin-Strep NifH: $35.3 \mathrm{kDa}$ and NifM: $32.3 \mathrm{kDa}$. (C) Western blot analysis of eGFP accumulation. Expected size: $27 \mathrm{kDa}$. (D) Quantification of Nif/eGFP accumulated protein normalized to native $A$. vinelandii sequence protein accumulation. Mean $\pm \mathrm{SD}(\mathrm{n}=3$ for $\mathrm{NifH}, \mathrm{n}=2$ for $\mathrm{NifM}$ ). The given value for each Nif protein band was normalized using the value corresponding to the eGFP signal in the same lane. Means not sharing any letter are significantly different by Tukey's multiple comparisons test at the $95 \%$ level of significance. 
In the case of NifU, antibodies available in our laboratory presented a pattern of unspecific bands that made its detection by immunoblot impossible (Figure 20B). Regarding NifS, no signal could be detected at the expected size for any of the optimizations, although the eGFP internal control present in each plasmid was detected in all cases, indicating that transformation worked properly and that NifS accumulation levels were too low for detection (Figure 20B, C).

(A)

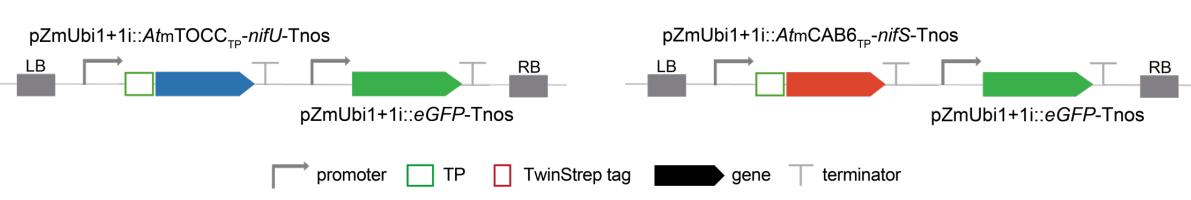

(B)

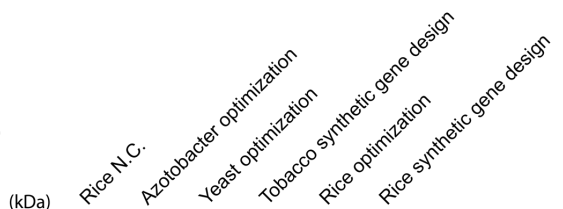

(C)
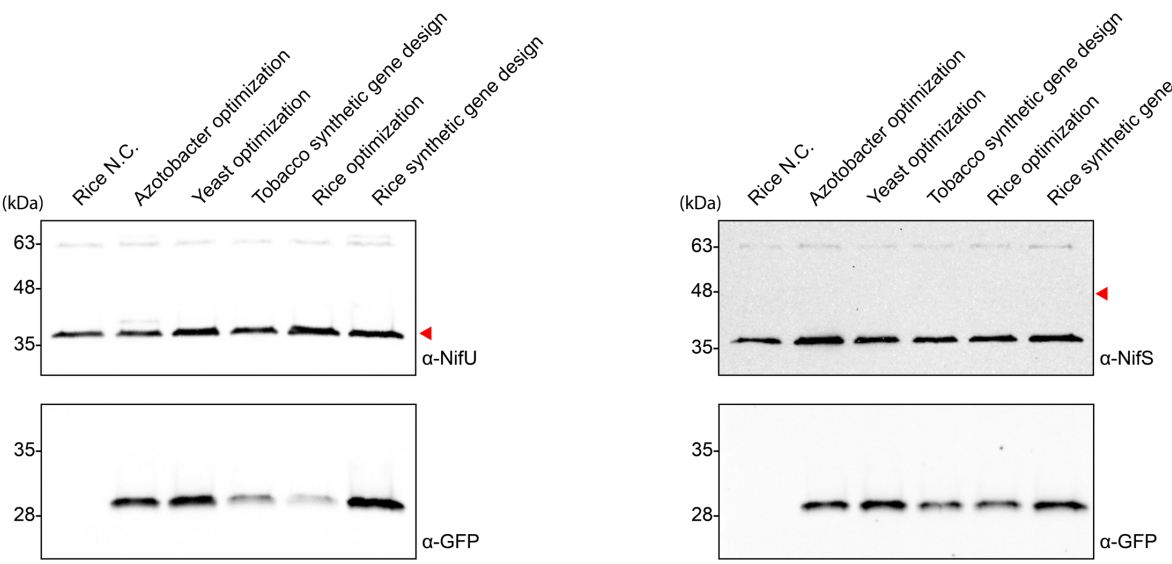

Figure 20. nif $U$ and nifS gene optimizations led to no detectable protein in rice.

(A) Schematic representation of the constructs used in $O$. sativa-transient expression assays leading to nif $U$ or nifS gene and eGFP simultaneous expression. (B) Rice protoplasts western blot analysis of NifU (left) and NifS (right) accumulation when expressed from its native $A$. vinelandii gene, a yeast codonoptimized sequence, a synthetically designed gene optimized for expression in tobacco, a rice codonoptimized sequence, or a synthetically designed gene optimized for expression in rice. N.C. refers to non-transformed protoplasts. The triangles in red mark the expected mature form of the protein. An intense $35 \mathrm{kDa}$ non-specific band can be observed when using $\alpha$-NifU and $\alpha$-NifS antibodies. Expected sizes are NifU: $34.4 \mathrm{kDa}$ and NifS: $44.3 \mathrm{kDa}$. (C) Western blot analysis of eGFP accumulation. Expected size: $27 \mathrm{kDa}$.

\subsubsection{Import plastid-type specificity}

We had previously validated the minimal versions of four Arabidopsis TPs that were able to direct Nif recombinant proteins from bacterial origin to tobacco chloroplasts (Section 7.2.2). In an effort to gauge the utility of these TPs as tools for cereal engineering, we decided to study their species and plastid specificity.

With that purpose, we tested the efficiency of the previously selected four minimal Arabidopsis TPs, which come from proteins directly involved in photosynthesis (CAB6, chlorophyll a/b binding protein; $\mathrm{RCA}, \mathrm{RuBisCO}$ activase), photo-protection (TOCC, tocopherol cyclase), and fatty acid biosynthesis (BCCP1, Biotin carboxyl carrier protein) 
and of one complete TP involved in the assimilation of ammonium into amino-acids (GLTB2, ferredoxin dependent glutamate synthase 2) (Figure 21A and Supplemental Figure 5). CAB6, RCA, TOCC, and BCCP1 are highly expressed in green tissue (Figure 21B, left panel), and accumulate preferentially in leaves (Figure 21B, right panel), while GLTB2 expression and protein accumulation are most abundant in roots, as evidenced by data from the Plant eFP browser (Waese et al., 2017) and AtProteome (Baerenfaller et $a l ., 2008)$ databases. It is interesting to note that TOCC was also expressed at low levels in roots, consistent with recent studies that suggest a role of tocopherols in the adaptation to drought (Cela et al., 2011) and heavy metals (Collin et al., 2008).

(A)

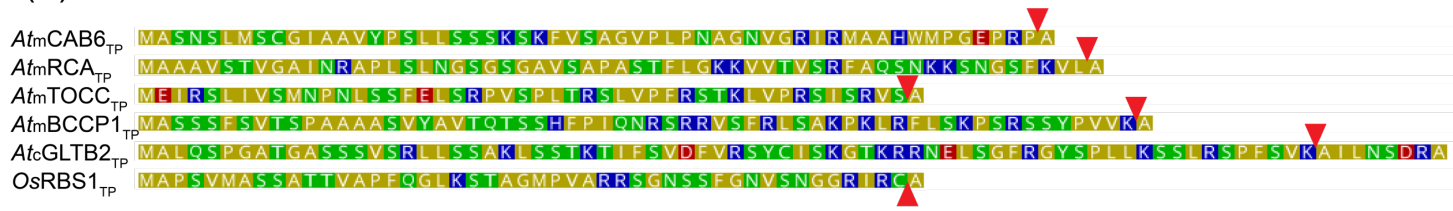

(B)

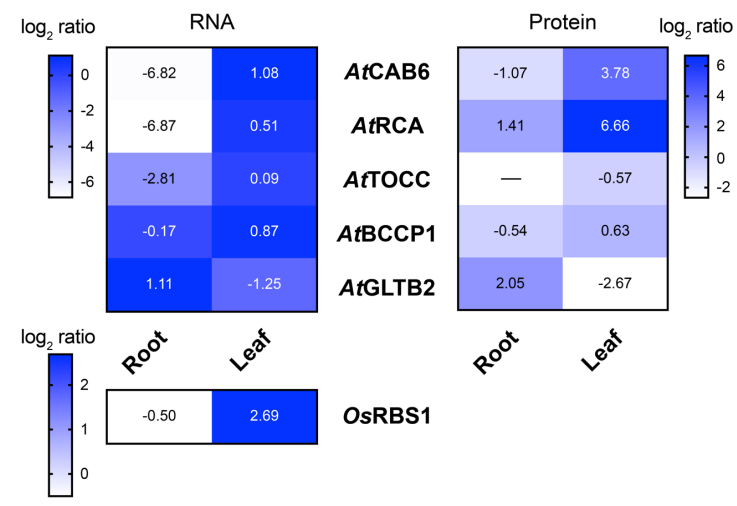

Figure 21. Transit Peptides from A. thaliana and $O$. sativa.

(A) Amino acid sequence of Transit Peptides (TPs) from A. thaliana and $O$. sativa. Colouring according to amino acid polarity as follows: Yellow: Non-polar; Green: Polar, uncharged; Red: Polar, acidic; Blue: Polar, basic. Red arrows indicate the predicted Stromal Processing Peptidase (SPP) cleavage site. (B) Gene expression and protein levels of corresponding TP genes and mature proteins in A. thaliana and $O$. sativa. Gene expression data were retrieved from the Plant eFP browser, showing $\log _{2}$ of fold change from microarray transcriptomics (Waese et al., 2017). Protein data were retrieved from AtProteome (Baerenfaller et al., 2008). Heatmap shows the $\log _{2}$ ratio from shotgun proteomics.

We generated constructs in which these five Arabidopsis TPs together with a rice TP coming from the Rubisco small chain 1 protein $\left(O s \mathrm{RBS} 1_{\mathrm{TP}}\right)$, which is highly expressed in leaves (Figures 21A and B), were fused to the reporter protein eGFP and cytosolic eGFP was used as control of mature protein size (Figures 22A, 23A, and 25A). In order to analyse import mediated by the different TPs, protoplasts obtained from Arabidopsis mesophyll (Figures 22B, 24A, and Supplemental Figure 6) and root cells (Figure 23B, 25B, and Supplemental Figure 6) were transformed. 
(A)
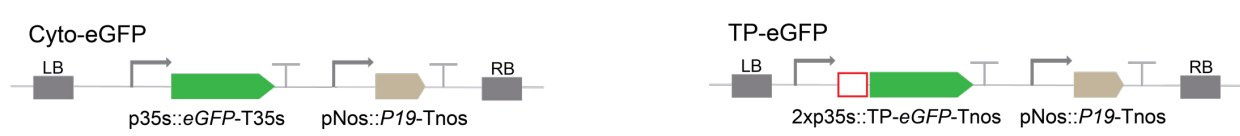

(B)

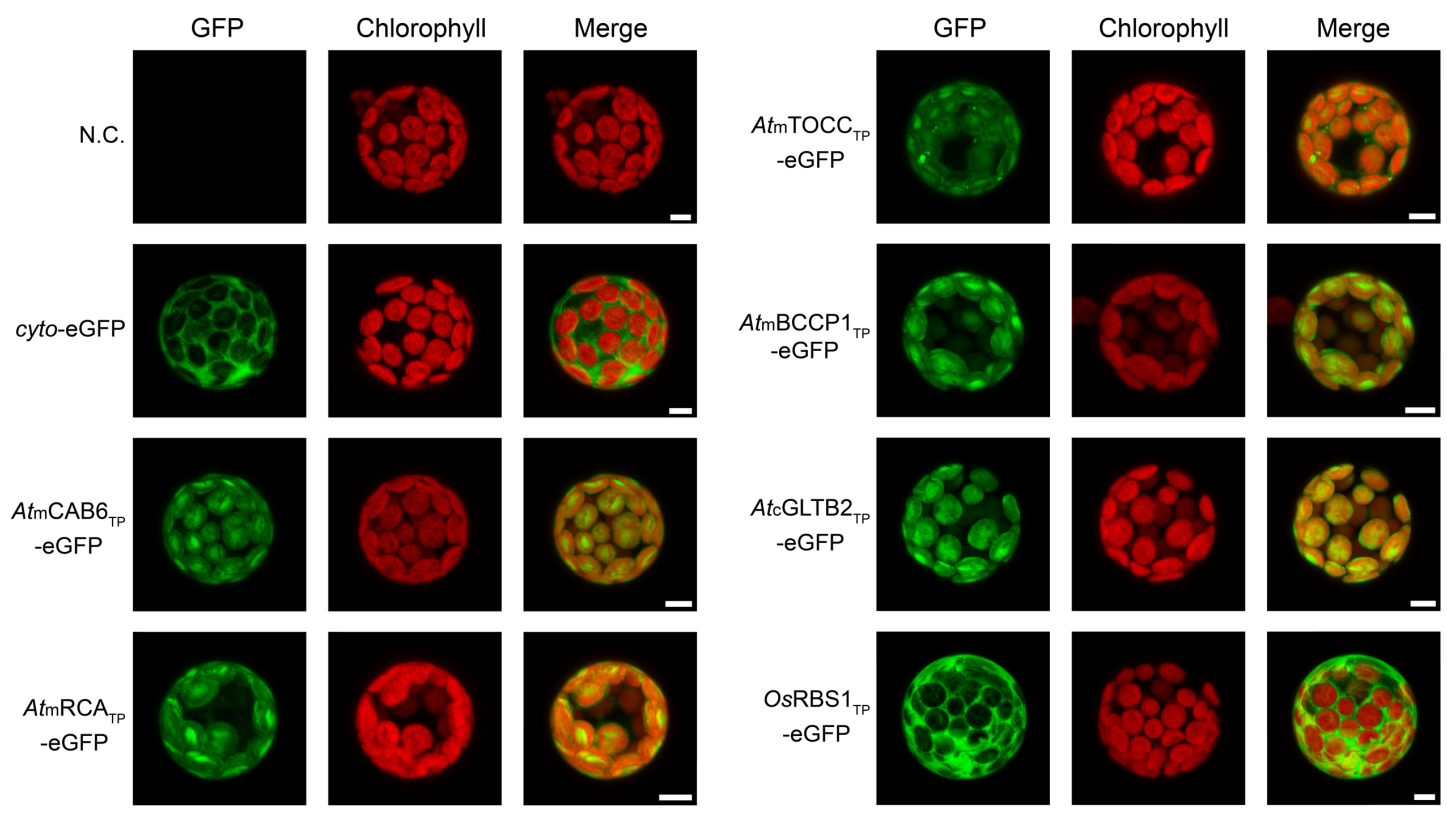

Figure 22. In vivo chloroplast targeting of TP-eGFP fusion proteins in Arabidopsis leaf protoplasts. (A) Schematic representation of the constructs used in A. thaliana transient expression assays leading to eGFP expression and localization. (B) Confocal laser scanning microscopy images of $A$. thaliana leaf protoplast expressing indicated TP-eGFP fusions. N.C. corresponds to "Negative Control", nontransformed protoplasts. The columns show individual signals for eGFP (green, on the left) and chlorophyll autofluorescence (red, in the centre). On the right, the overlap of both signals (merge). Scale bars $=5 \mu \mathrm{m}$.

Chloroplasts were easily identified in protoplasts of mesophyll cells by their red autofluorescence (Figure 22B). The plastid-targeted eGFP coincided with chlorophyll fluorescence for all Arabidopsis TPs, whereas for the rice OsRBS1 TP TP and the cytosolic control, eGFP fluorescence was localized outside the chloroplasts (Figure 22B). These results are consistent with our previous observations in tobacco (Section 7.2.2) and demonstrate that the selected Arabidopsis TP sequences contain all necessary motifs to correctly direct import of nuclear-encoded recombinant proteins into chloroplasts. 
(A)

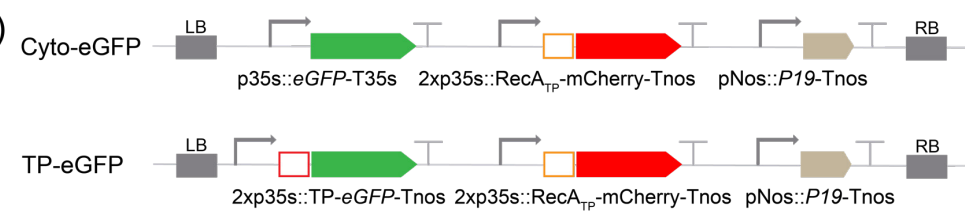

(B)

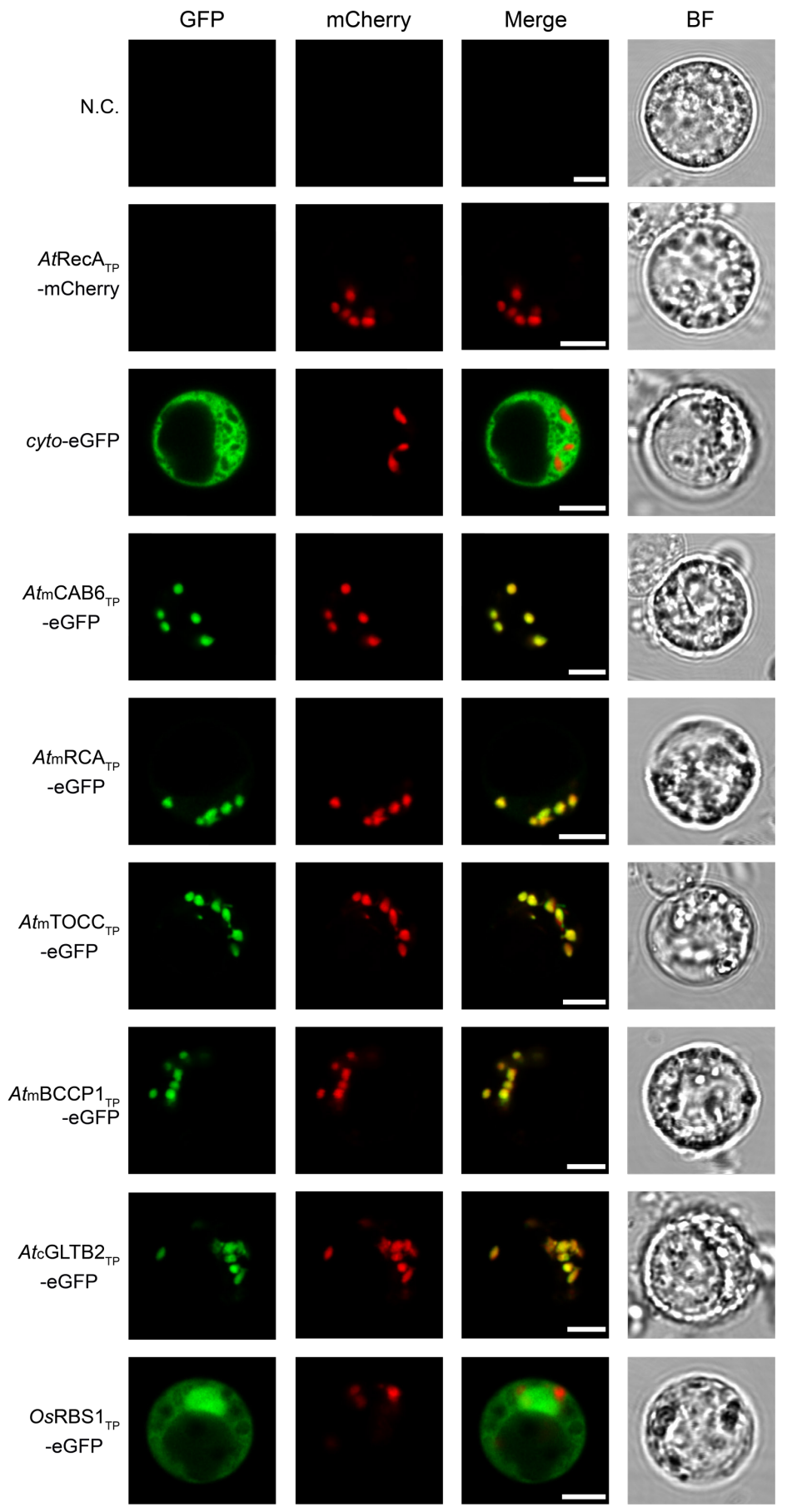

Figure 23. In vivo leucoplast targeting of TP-eGFP fusion proteins in Arabidopsis root protoplasts. (A) Schematic representation of the constructs used in A. thaliana transient expression assays leading to eGFP expression and localization. (B) Confocal laser scanning microscopy images of $A$. thaliana root protoplast expressing indicated TP-eGFP fusions. N.C. corresponds to "Negative Control", nontransformed protoplasts. The four columns show individual signals for eGFP (green, on the far left), mCherry (red, on the left), and transmitted light (BF, on the far right). On the right, the overlap of eGFP and mCherry signals (merge). Scale bars $=5 \mu \mathrm{m}$. 
Expression of the Arabidopsis TP-eGFP fusions in protoplasts obtained from Arabidopsis root cells rendered a fluorescence overlapping with that produced by the expression of AtRecA $\mathrm{AP}_{\mathrm{TP}}$-mCherry, an established root plastid marker (Nakata et al., 2018) and different to the one observed for the rice $O s \mathrm{RBS} 1_{\mathrm{TP}} \mathrm{TP}$ and the cytosolic control (Figure 23). This result suggests that the selected Arabidopsis TPs also mediate successful import of eGFP into root leucoplasts.

(A)
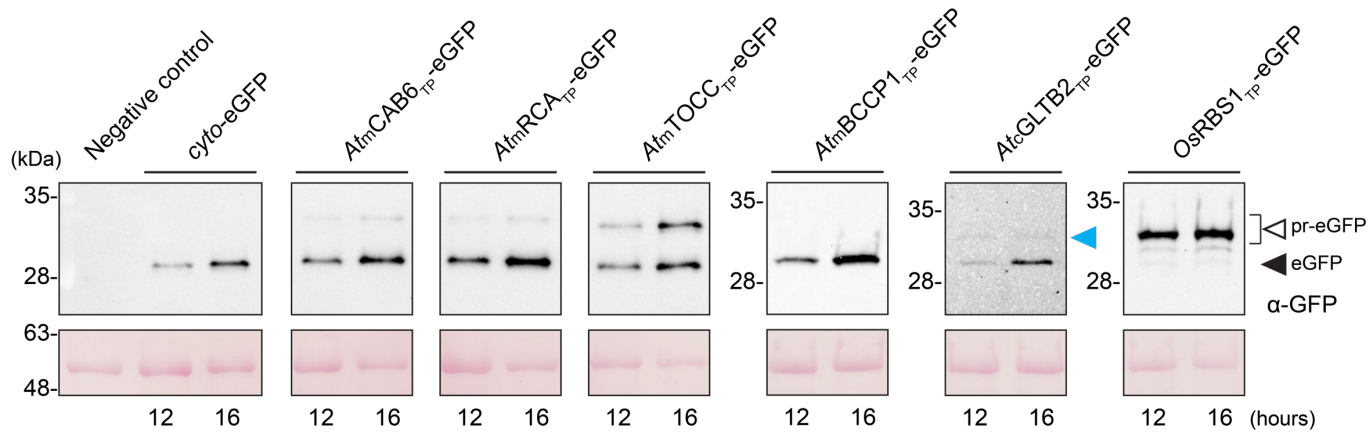

(B)

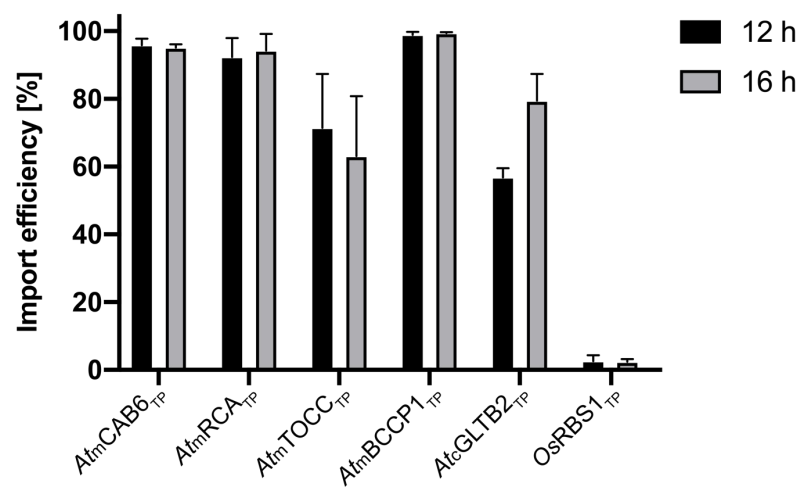

Figure 24. Import efficiency of $A$. thaliana and $O$. sativa Transit Peptides in Arabidopsis leaf protoplasts.

(A) Western-blot analysis of import experiments. Protoplast isolated from $A$. thaliana leaves were transformed with indicated TP-eGFP fusions and total protein extracts were analysed by Western blotting using an anti-GFP antibody. The experiments were performed in three biological replicates and representative data are shown. White arrows (pr-eGFP) indicate precursor form; black arrows (eGFP) indicate, processed form; blue arrow indicates partially processed form. (B) Quantification of import efficiency of TPs into Arabidopsis chloroplast. Import efficiency was defined as the percentage of the processed faster-migrating protein form relative to the total amount of expressed eGFP protein in each line. Data represent means $(n=3)$ with SD.

SDS-PAGE and immunoblot analysis of protein extracts from Arabidopsis mesophyll (Figure 24A) and root protoplasts (Figure 25A, B) taken at two different time points (12 and $16 \mathrm{~h}$ after transformation) made the evaluation of the dynamics of protein import possible. The results show that although some increase in total protein abundance could be observed after $16 \mathrm{~h}$, import efficiency is mostly stable and not dependent on time. The immunoblots showed the presence of lower mobility bands, in some cases, with a size 
corresponding to the pre-protein (pr-eGFP), in others, smaller, which could correspond to processing within the TP; and a higher mobility band with a size consistent with the processed eGFP protein after SPP cleavage during import. The ratio of processed to total protein in each lane provides an estimate of import efficiency for each TP in leaf and root plastids (Figure 24B and 25C, respectively).

(A)

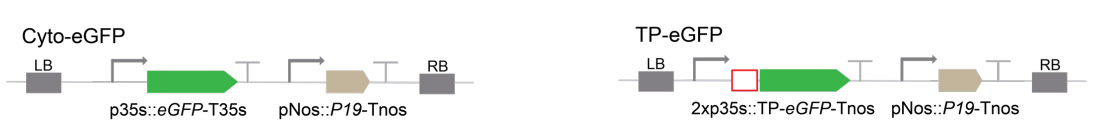

(B)

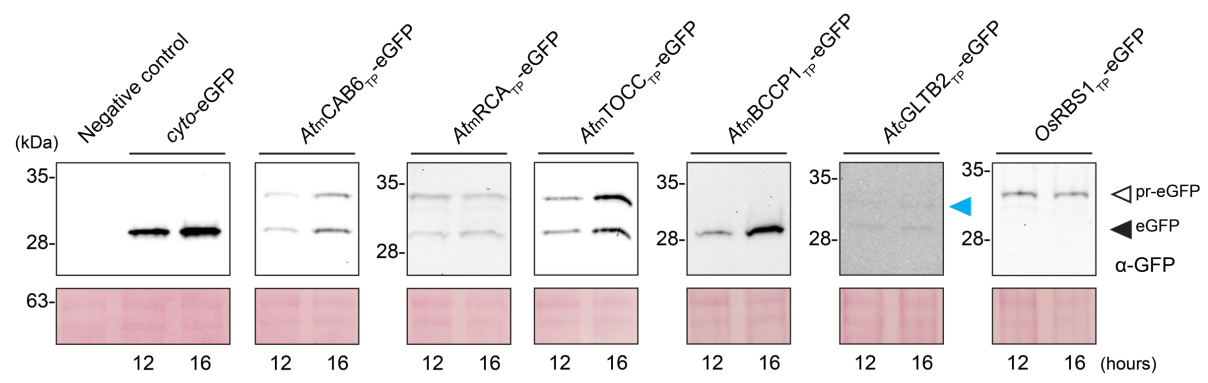

(C)

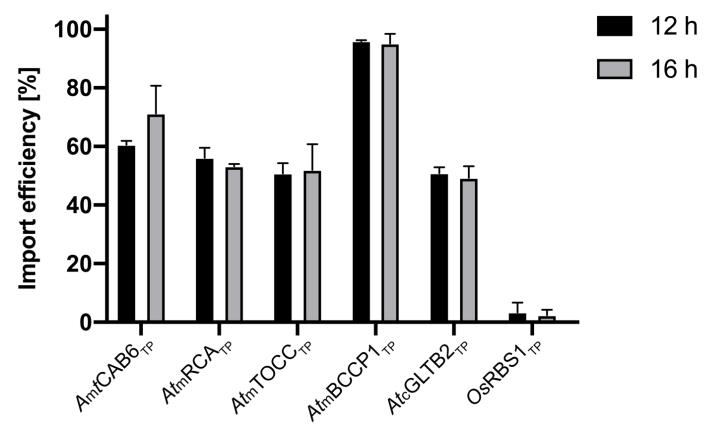

Figure 25. Import efficiency of $A$. thaliana and $O$. sativa Transit Peptides in Arabidopsis root protoplasts.

(A) Schematic representation of the constructs used in A. thaliana transient expression assays leading to eGFP expression. (B) Western-blot analysis of import experiments. Protoplast isolated from $A$. thaliana roots were transformed with indicated TP-eGFP fusions and total protein extracts were analysed by Western blotting using an anti-GFP antibody. The experiments were performed in three biological replicates and representative data are shown. White arrows (pr-eGFP) indicate precursor form; black arrows (eGFP) indicate, processed form; blue arrow indicates partially processed form. (C) Quantification of import efficiency of TPs into Arabidopsis root plastids. Import efficiency was defined as the percentage of the processed faster-migrating protein form relative to the total amount of expressed eGFP protein in each line. Data represent means $(\mathrm{n}=3)$ with SD, except GLTB2 and RBS1 TPs, where $\mathrm{n}=2$.

Although some preference was noted for chloroplast import in the case of AtmCAB6TP (96\% and 95\%, at $12 \mathrm{~h}$ and $16 \mathrm{~h}$ respectively) and AtmRCA $\mathrm{TP}_{\mathrm{TP}}$ TPs (92\% and 94\%), both still showed relatively high efficiency in the import to root leucoplasts $(61 \%-70 \%$ and $56 \%-54 \%$, respectively). However, AtmBCCP1 $1_{\mathrm{TP}}$ exhibited the highest efficiency in the import to both chloroplast and root leucoplasts (99\% - 99\% and 97\% - 98\%, respectively) 
of the TPs set studied here. Conversely, AtmTOCC ${ }_{\text {TP }}$ and AtcGLTB2 ${ }_{\text {TP }}$ TPs showed medium import efficiency into chloroplasts $(71 \%-63 \%$ and $57 \%-79 \%)$, and also a bit lower in leucoplasts $(51 \%-52 \%$ and $51 \%-49 \%$, respectively). This is a surprising result for $A t c G L T B 2$ TP, given that its actual function is performed in the root tissue where it is preferentially expressed. Nevertheless, possible processing within the AtcGLTB2 ${ }_{\text {TP TP }}$ resulted in an additional band, decreasing its import efficiency. The rice OsRBS1 TP TP was unable to correctly target eGFP into chloroplasts $(2 \%-2 \%$, at $12 \mathrm{~h}$ and $16 \mathrm{~h}$ respectively) or leucoplasts $(3 \%-2 \%)$ of Arabidopsis, accumulating as a pre-protein (Figure 24A and 25B), and suggesting a species-specific import mechanism. For full data and statistics, please see Supplemental Table 12.

\subsubsection{Conservation of the import mechanism between species}

The identification of Arabidopsis TPs that mediate effective plastid import of recombinant proteins in both, Arabidopsis AtmCAB6тP, AtmRCATP, AtmTOCC $_{\mathrm{TP}}$, $A t \mathrm{mBCCP} 1_{\mathrm{TP}}$ and $A t \mathrm{cGLTB} 2_{\mathrm{TP}}$ ) and tobacco $\left(\right.$ AtmCAB6$_{\mathrm{TP}}, A t \mathrm{mRCA} \mathrm{TP}_{\mathrm{TP}}$ AtmTOCC $\mathrm{TP}_{\mathrm{TP}}$ and $A t \mathrm{mBCCP} 1_{\mathrm{TP}}$, Section 7.2.2), and of a rice TP that is not able to mediate import in Arabidopsis $\left(O s \mathrm{RBS} 1_{\mathrm{TP}}\right)$ provided a basis for further investigation. Therefore, we deepened our study on the extent of conservation of plastid translocation mechanisms between Arabidopsis and rice. To this end, eGFP constructs analogous to those described above were designed for expression in monocot cells (Figure 26A) and used to transform protoplasts of cells from rice sheath and stem (Supplemental Figure 6). The plastidtargeted eGFP signal coincided with chlorophyll fluorescence in all cases, in contrast to that of the cytosolic eGFP (Figure 26B), confirming that all the selected TPs were functional in rice cells, as opposed to previous results in Arabidopsis. 
(A)

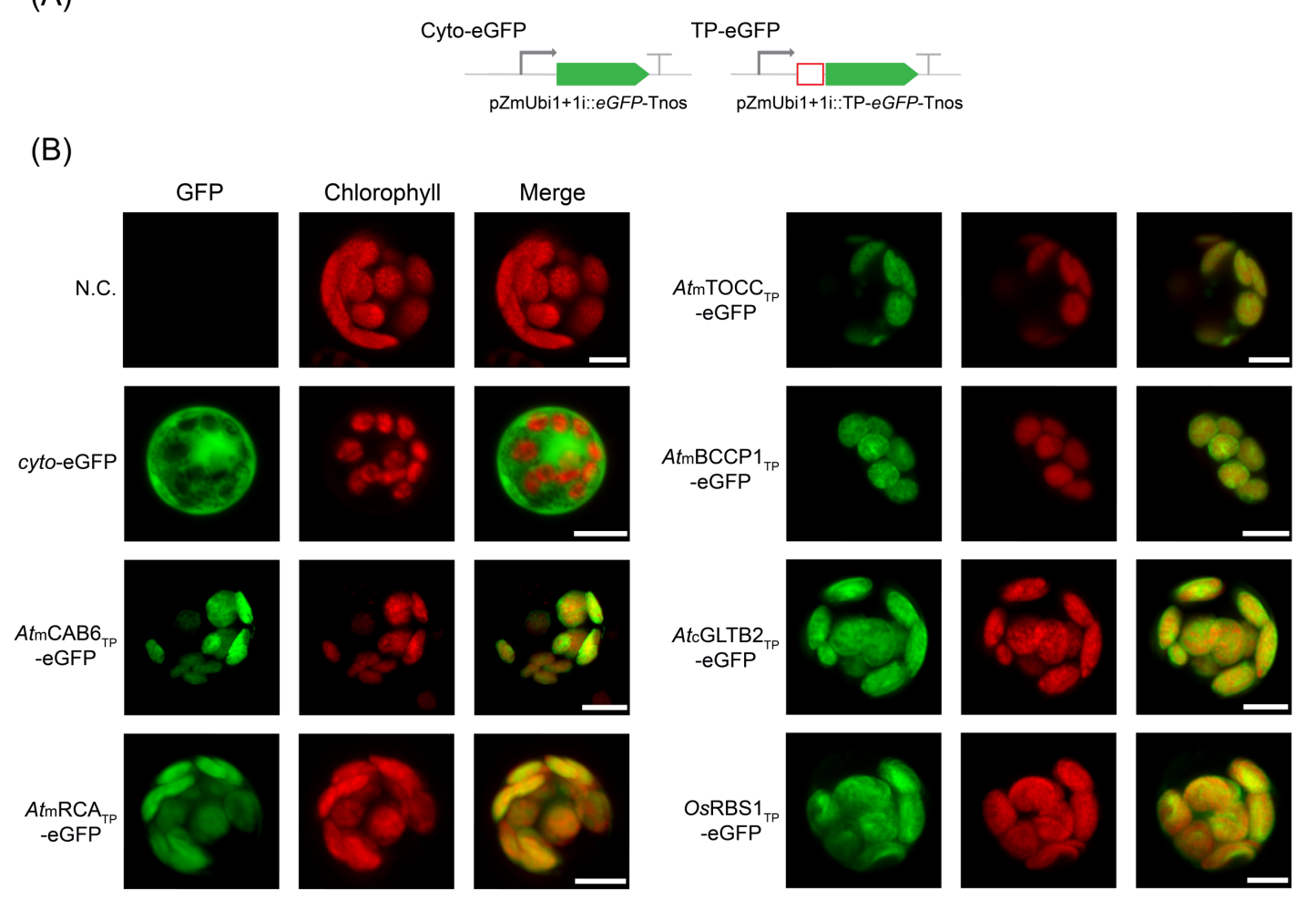

Figure 26. Arabidopsis TPs are able to direct eGFP into rice chloroplast.

(A) Schematic representation of the constructs used in $O$. sativa transient expression assays leading to eGFP expression and localization. (B) Confocal laser scanning microscopy images of rice protoplast expressing indicated TP-eGFP fusions. N.C. corresponds to "Negative Control", non-transformed protoplasts. The three columns show individual signals for eGFP (green, on the left), chlorophyll autofluorescence (red, in the centre), and overlap of both signals (right). Scale bars $=5 \mu \mathrm{m}$.

SDS-PAGE and immunoblot analysis of transformed rice protoplast extracts showed a lower mobility band corresponding to pre-eGFP and a higher mobility band corresponding to processed eGFP (Figure 27A). Interestingly, import efficiencies observed were, in the case of $A t \mathrm{mCAB} 6_{\mathrm{TP}}(80 \%-81 \%$, at $12 \mathrm{~h}$ and $16 \mathrm{~h}$ respectively) and $A t \mathrm{mRCA}_{\mathrm{TP}}(85 \%-88 \%)$, slightly lower than those observed for Arabidopsis chloroplasts; higher in the case of $A t m$ TOCC 99\%); and equal for $A t \mathrm{mBCCP} 1_{\mathrm{TP}}(94 \%$ - 98\%) (Figure 27B). The rice endogenous OsRBS1 $1_{\mathrm{TP}}$ was now able to direct eGFP into chloroplasts, but, surprisingly, less efficiently $(68 \%-68 \%$, at $12 \mathrm{~h}$ and $16 \mathrm{~h}$ respectively) than its counterparts from Arabidopsis. For full data and statistics, please see Supplemental Table 12. 
(A)

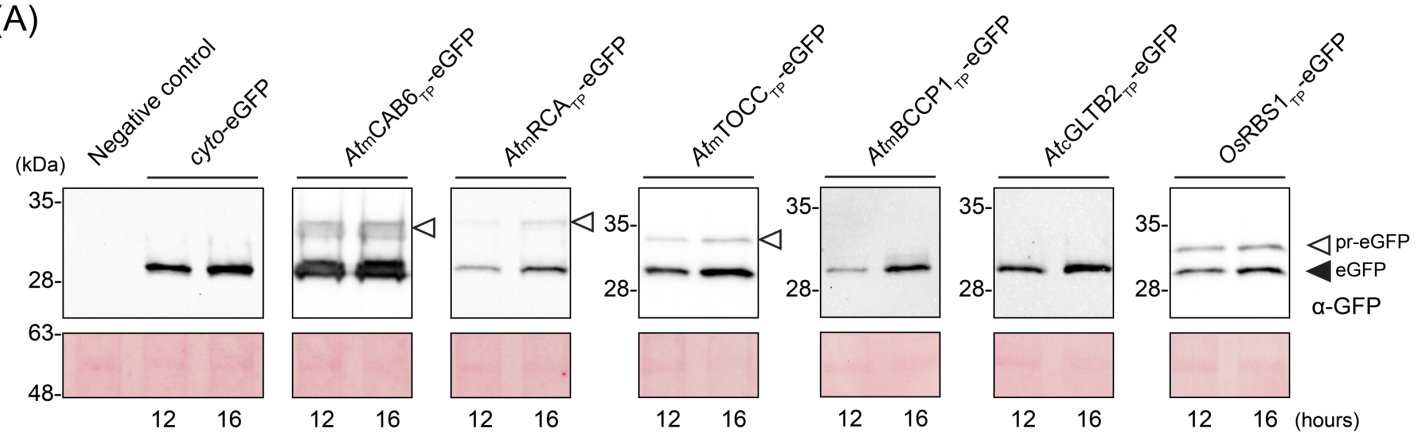

(B)

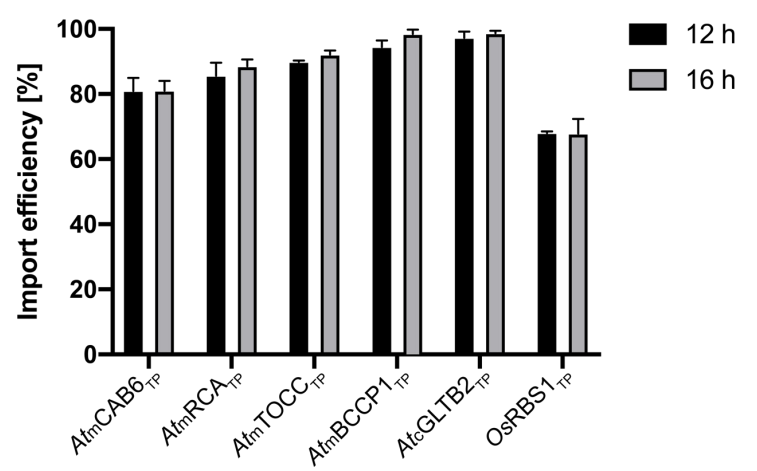

Figure 27. Import efficiency of $A$. thaliana and $O$. sativa Transit Peptides in rice protoplast.

(A) Western-blot analysis of import experiments. Protoplasts isolated from rice were transformed with indicated TP-eGFP fusions and total protein extracts were analysed by western blotting using an antiGFP antibody. The experiments were performed in three biological replicates and representative data are shown. White arrows (pr-eGFP) indicate precursor form; black arrows (eGFP) indicate, processed form. (B) Quantification of import efficiency of TPs into rice chloroplast. Import efficiency was defined as the percentage of the processed faster-migrating protein form relative to the total amount of expressed eGFP protein in each line. Data represent means $(n=3)$ with SD.

\subsubsection{Arabidopsis TPs as tools for plastid import in whole rice plants}

In light of the previous results supporting the efficiency of Arabidopsis TPs in rice green tissue, we generated transgenic rice lines in which rice embryos were co-transformed with a plasmid carrying a hygromycin selection cassette and the AtmCAB6тP, AtmRCATP, AtmTOCC ${ }_{\mathrm{TP}}$, and AtmBCCP1 $1_{\mathrm{TP}}$ TPs fused to the N-terminus of eGFP (Figure 28A). Several independent embryogenic callus lines were recovered from each combination of plasmids in which eGFP was expressed to such levels as to be visible under the stereomicroscope (Figure 28B). Lines recovered after transformation with the construct containing the AtmTOCC $\mathrm{TP}$ TP had lower expression levels compared to AtmCAB6TP, AtmRCATP, or $A t \mathrm{mBCCP} 1_{\mathrm{TP}}$ TPs (as evidenced by fluorescence emission and protein accumulation). Immunoblot analyses confirmed in all four cases, that only the fully cleaved version of eGFP was detected in callus proplastids, indicating almost $100 \%$ import efficiency (Figure 28C, Supplemental Table 12). 
(A)

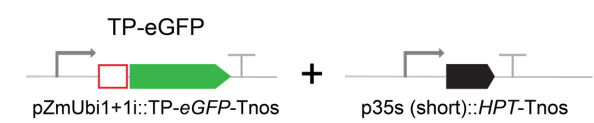

(C)
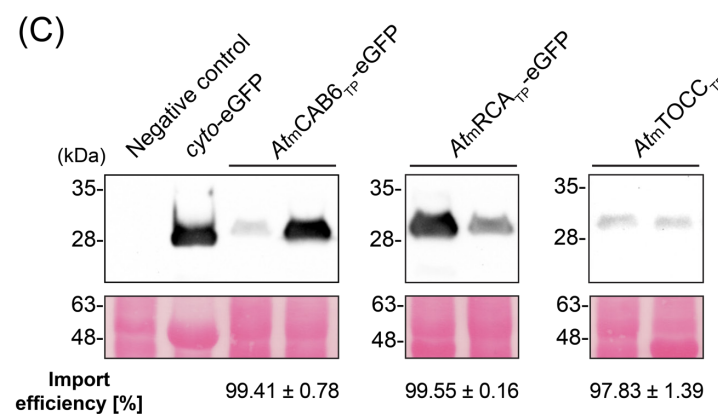

$97.83 \pm 1.39$

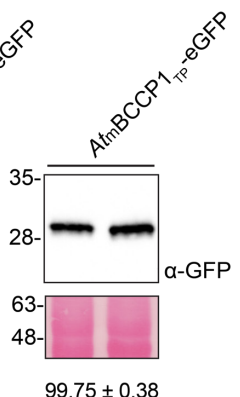

$99.75 \pm 0.38$
(B)

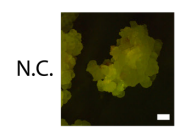

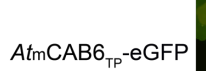

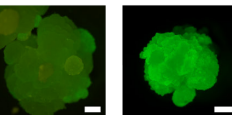

$A_{\text {mRCA }}$ TP - eGFP

.

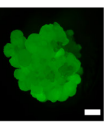

AtmTOCC $_{\mathrm{TP}}$-eGFP

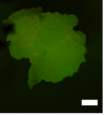

23

$A t_{\mathrm{mBCCP}} 1_{\mathrm{TP}}$-eGFP

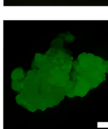

Figure 28. Stably transformed rice lines accumulate eGFP in callus stage.

(A) Schematic representation of the constructs used for stable transformation of $O$. sativa leading to eGFP expression and localization. (B) Stereomicroscope fluorescence images from two independent transformed callus lines expressing AtmCAB6 TP-eGFP, AtmRCA $_{\mathrm{TP}}-\mathrm{eGFP}$, AtmTOCC $\mathrm{TP}_{\mathrm{TP}}-\mathrm{eGFP}$ or AtmBCCP1 $1_{\mathrm{TP}}$-eGFP. N.C. corresponds to "Negative Control", a transformed callus line expressing only the HPT resistance cassette. Scale bars $=1 \mathrm{~mm}$. (C) Western blot analysis of callus soluble protein extracts from two independent transformed rice lines with the indicated constructs incubated with an anti-GFP antibody. eGFP produced in $N$. benthamiana was used as a positive size control. Import efficiency was defined as the percentage of the processed faster-migrating protein form relative to the total amount of expressed eGFP protein. Data shown are means \pm SD of three independent extractions.

Localization of eGFP within callus proplastids was confirmed using immunogold labelling and electron microscopy. When using a monoclonal antibody against eGFP, callus lines showed very low levels of labelling, but a very specific association of particles with the interior of plastids (Figure 29). Labelling using polyclonal antibodies against eGFP produced significant non-specific labelling, also visible in the wild-type callus, especially in nuclei and cytoplasm, but also higher signal density could still be observed within plastids (Supplemental Figure 7), consistent with our earlier results (Figure 29). An estimated quantification of the immunogold labelling results can be found in Supplemental Table 13.

Whole transgenic rice plants could be regenerated from the eGFP expressing callus lines for $A t \mathrm{mCAB} 6_{\mathrm{TP}}, A t \mathrm{mRCA} \mathrm{TP}_{\mathrm{TP}}$, and $A t \mathrm{mTOCC} \mathrm{TP}_{\mathrm{TP}}$ TPs to study the Arabidopsis TPs import efficiency in different rice tissues. All attempts to regenerate $A t m B C C P 1_{\text {TP }}$ TP lines resulted in embryogenic callus death. In leaves, immunoblot analysis revealed an almost 100\% import efficiency for all three TPs (Figure 30A, Supplemental Table 12), much higher than the one observed in the protoplast transient expression system (Figure 27). Electron microscopy immunogold detection showed labelling of chloroplasts, easily identifiable by their thylakoid structures, both by monoclonal (Figures 30B, C, D, and E) and polyclonal antibodies (Supplemental Figure 7). An estimated quantification of the immunogold labelling results can be found in Supplemental Table 13. Furthermore, 
confocal microscopy analysis showed co-localization of the eGFP signal with chlorophyll autofluorescence (Supplemental Figure 8), supporting correct plastid targeting mediated by the TPs in study.

(A)

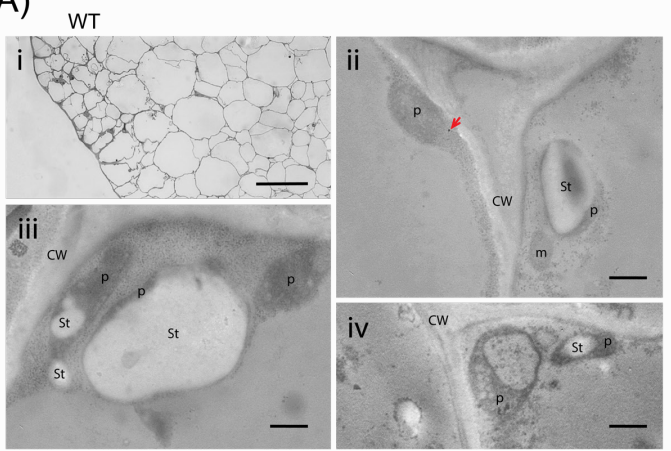

(B) ${ }_{A t m C A B G_{T P}-\text { eGFP }}$

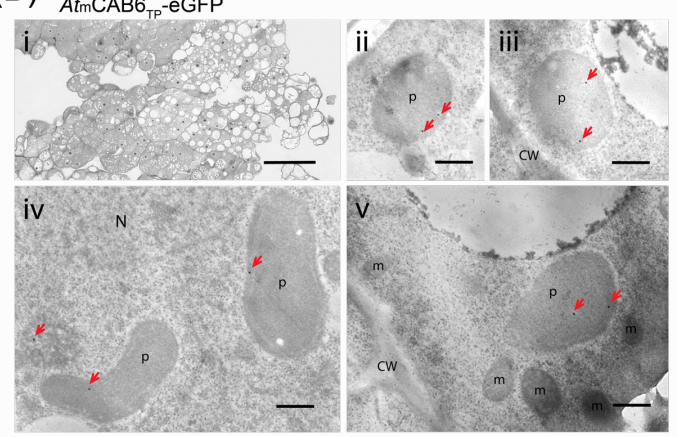

(C)

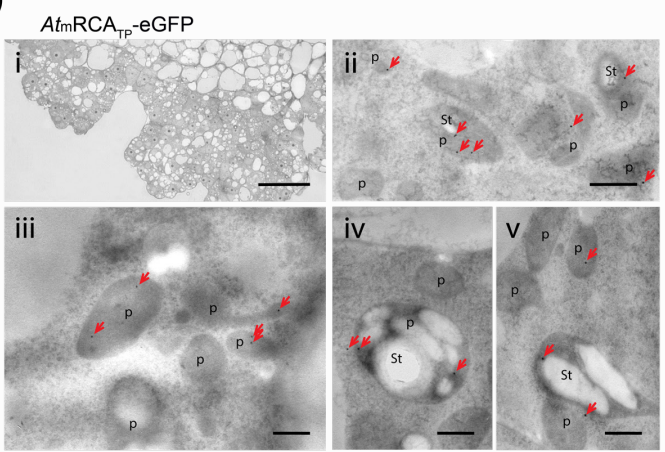

(D)

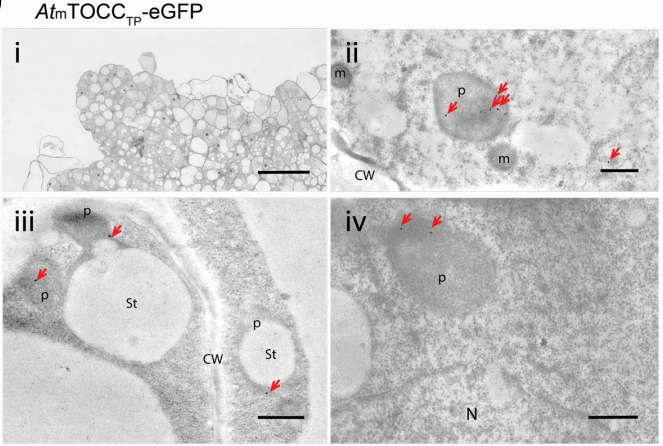

(E)

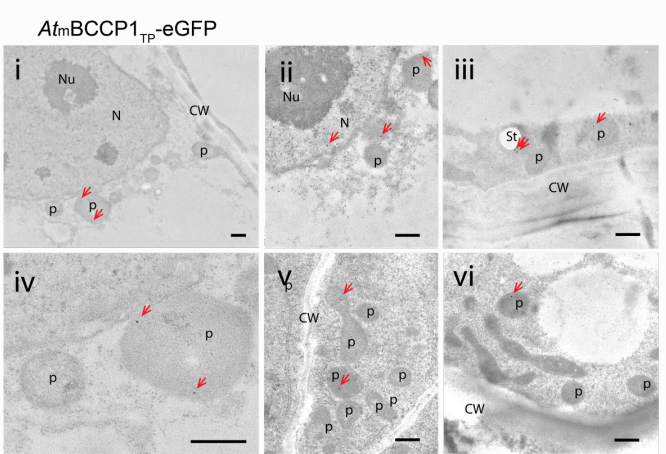

Figure 29. Stably transformed rice callus lines exhibit eGFP targeting into rice pro-plastids by Arabidopsis TPs.

(A-E) Immunogold labelling of eGFP in proplastids of rice callus cells using a GFP-specific monoclonal antibody (diluted 1:250). A Wild-type cells. B AtmCAB6 ${ }_{T P}-\mathrm{eGFP}$. C AtmRCATP-eGFP. D AtmTOCC TP- $_{\text {T }}$ eGFP. E $A t m B C C P 1_{\mathrm{TP}}$-eGFP. i: Light microscopy (LM) image of studied section, except in E; ii-v: Transmission electron microscope (TEM) images. (CW: cell wall, m: mitochondria, N: nucleus, p: plastid like, St: starch; bars i $=50 \mu \mathrm{m}, \mathrm{ii}-\mathrm{v}=500 \mathrm{~nm}$; gold particle $\mathrm{size}=15 \mathrm{~nm}$ ). 
(A)

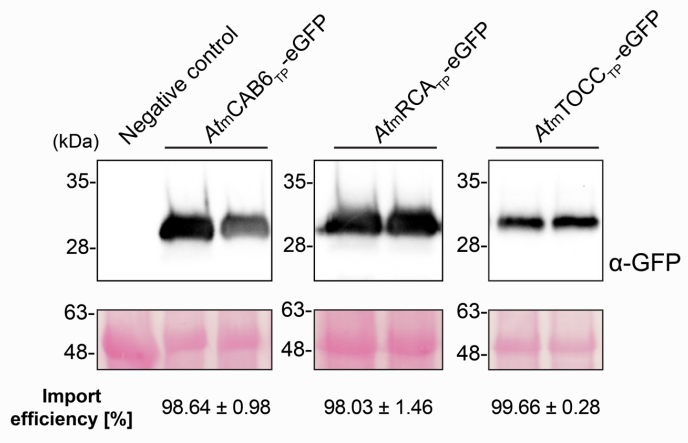

(B)

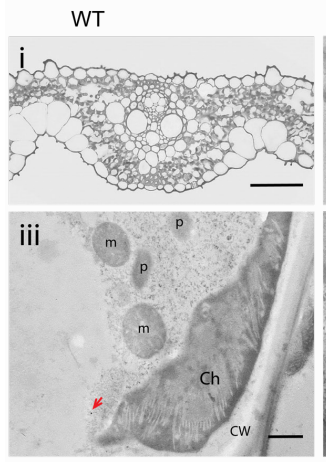

(D)
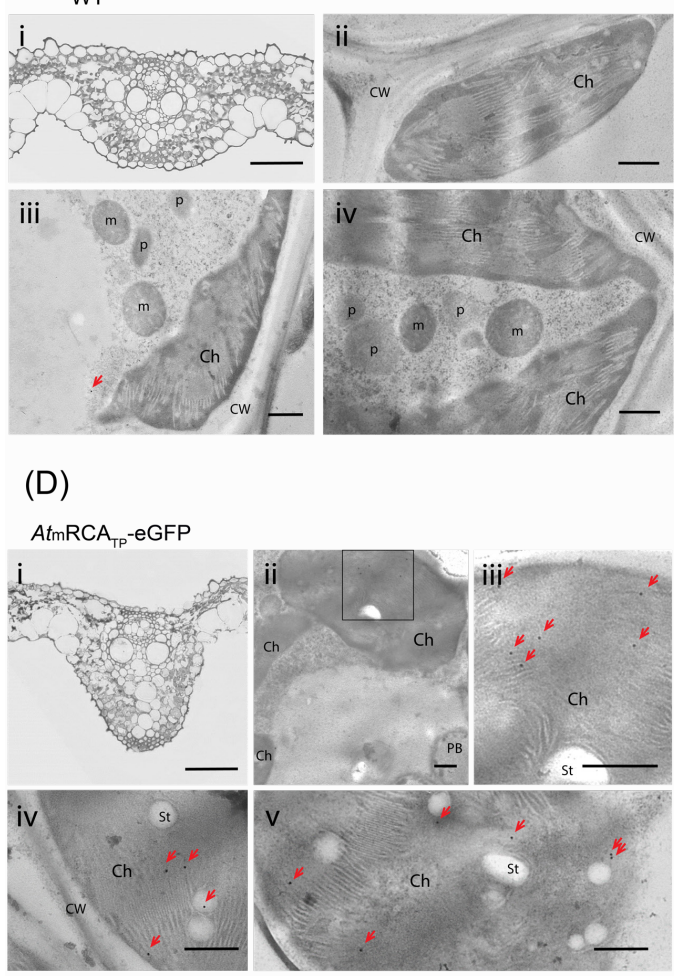

(C)

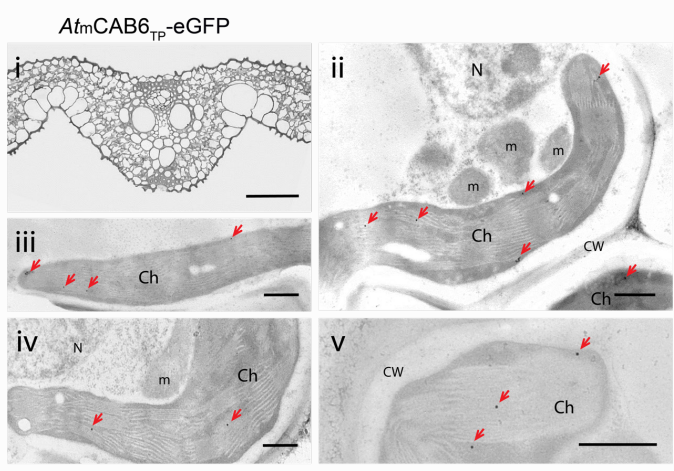

(E)

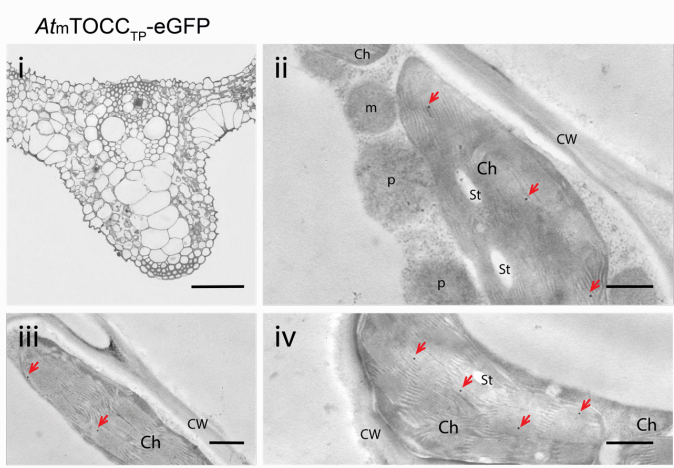

Figure 30. Regenerated rice plants show eGFP targeting into chloroplast driven by Arabidopsis TPs.

(A) Western blot analysis of leaf total protein extracts from two plants regenerated from the same transformed callus line expressing the indicated constructs above, incubated with an anti-GFP antibody. Negative Control represents a wild-type plant. Import efficiency was defined as the percentage of the processed faster-migrating protein form relative to the total amount of expressed eGFP protein. Data shown are means $\pm \mathrm{SD}$ of three independent extractions. (B-E) Immunogold labelling of eGFP in chloroplasts of rice leaf cells using a GFP-specific monoclonal antibody (diluted 1:250). B Wild-type cells. C $A t m C A B 6_{T P}-\mathrm{eGFP}$. D AtmRCA $\mathrm{TP}_{\mathrm{TP}}$-eGFP. E AtmTOCC $_{\mathrm{TP}}$-eGFP. i: Light microscopy (LM) image of studied section; ii-v: Transmission electron microscope (TEM) images. (Ch: chloroplast, CW: cell wall, m: mitochondria, $\mathrm{N}$ : nucleus, p: plastid like organelle, St: starch; bars $\mathrm{i}=50 \mu \mathrm{m}$, ii-v $=500$ $\mathrm{nm}$; gold particle size $=15 \mathrm{~nm}$ ).

Similar import efficiency was observed in roots of transgenic rice plants. A single band corresponding to mature eGFP was observed for the three TPs in their respective immunoblots, indicating again almost 100\% import efficiency (Figure 31A, Supplemental Table 12). Immunogold labelling using monoclonal antibodies showed weaker signals than in leaves or callus, but the gold particles were localized within 
leucoplasts (Figures 31B, C, D, and E). Polyclonal antibodies rendered non-specific labelling (also visible in the wild-type roots, especially in the cytoplasm) but the highest signal density was observed within leucoplasts (Supplemental Figure 7). For an estimated quantification of the signal, see Supplemental Table 13.

Taken together these results support the use of any of these TPs, AtmCAB6 ${ }_{\mathrm{TP}}$, AtmRCATP $_{\mathrm{m}}$ and $A t \mathrm{mTOCC}$ TP as tools for the engineering of rice chloroplasts or leucoplasts.

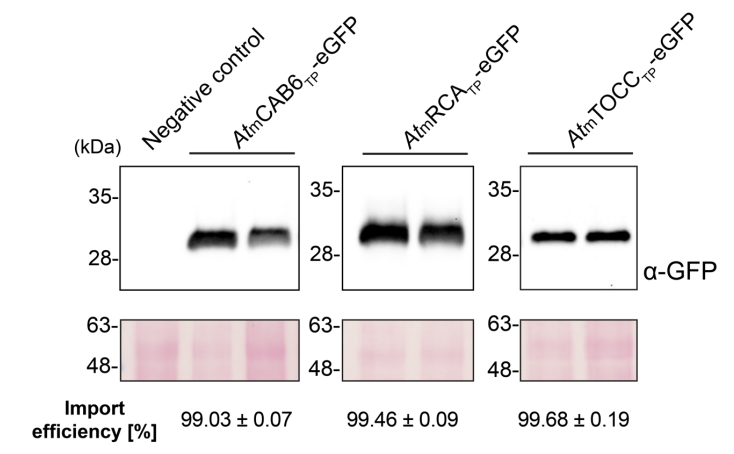

(B)

WT

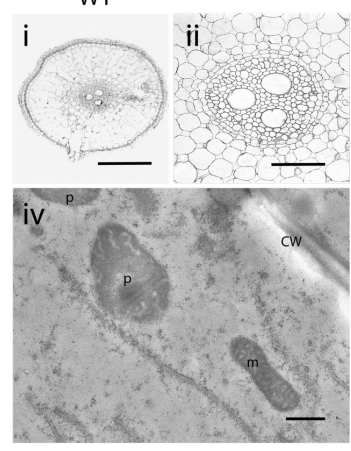

(D)

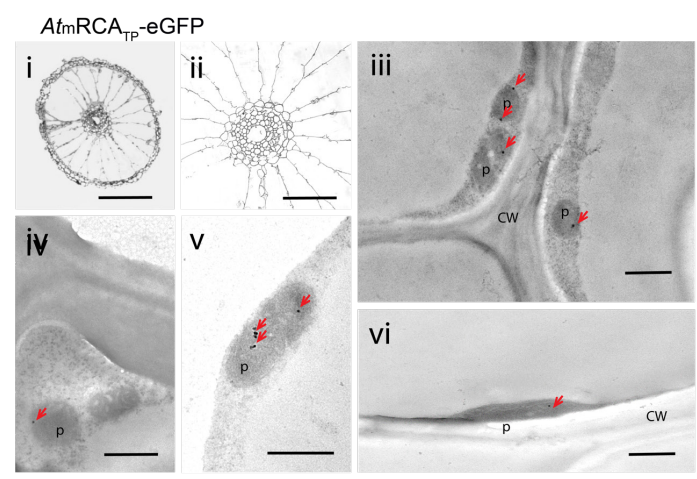

(C)

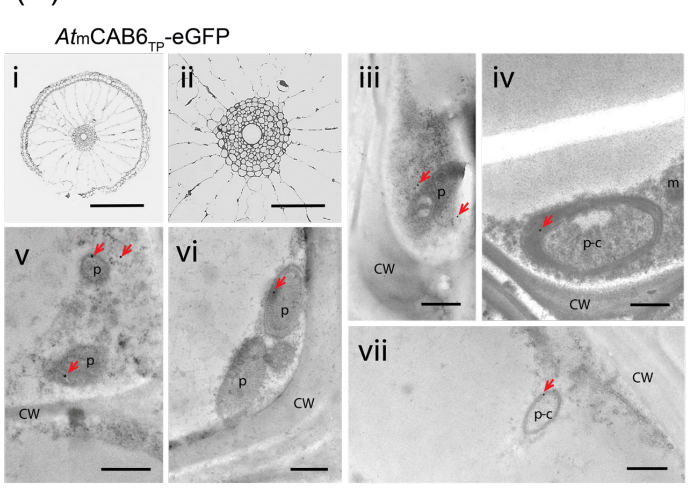

(E)

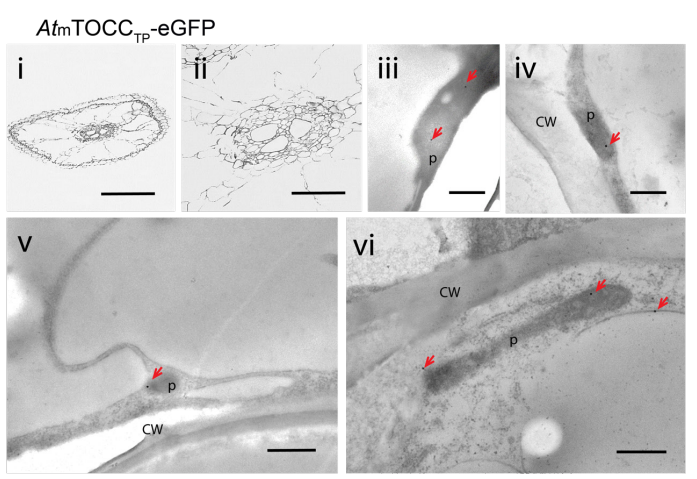

Figure 31. Roots from regenerated rice plants show eGFP targeting into leucoplasts driven by Arabidopsis TPs.

(A) Western blot analysis of root total protein extracts from two plants regenerated from the same transformed callus line expressing the indicated constructs above, incubated with an anti-GFP antibody. Negative Control represents a wild-type plant. Import efficiency was defined as the percentage of the processed faster-migrating protein form relative to the total amount of expressed eGFP protein. Data shown are means \pm SD of three independent extractions. (B-E) Immunogold labelling of eGFP in plastids of rice root cells using a GFP-specific monoclonal antibody (diluted 1:250). B Wild-type cells. C

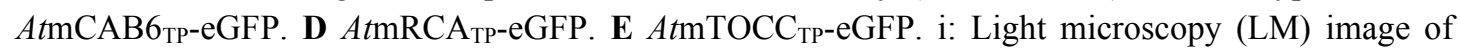


studied section; ii: detail of i (central cylinder); iii-vii: Transmission electron microscope (TEM) images. (CW: cell wall, m: mitochondria, $\mathrm{N}$ : nucleus, $\mathrm{Nu}$ : nucleolus, $\mathrm{p}$ : plastid-like, $\mathrm{p}$-c: plastid showing an isolated cytoplasmic region; bars $\mathrm{i}=0.2 \mathrm{~mm}, \mathrm{ii}=50 \mu \mathrm{m}$, iii-vii $=500 \mathrm{~nm}$; gold particle size $=15 \mathrm{~nm}$ ).

\subsubsection{NifM remains a requirement for $\mathrm{NifH}$ solubility in rice}

Following the optimization pipeline implemented in our work on tobacco, we next focused on evaluating the role and functionality of NifM in rice cells. For that purpose, we generated constructs consisting of $A t m R C A$ TP-NifH-tGFP and $A t m B C C P 1_{\text {TP-NifM- }}$ mCherry fusions, in which the expression of the rice codon-optimized nifH and nif $M$ genes were driven by the pZmUbil promoter. We used them to transiently transform rice sheath and stem protoplast (Supplemental Figure 6) and visualize tGFP and mCherry signals to assess their subcellular localization after individual and co-expression of $\mathrm{NifH}$ with NifM proteins (Figure 32A).

The individual expression of NifM showed a diffuse pattern colocalizing with chlorophyll (Figure 32B), suggestive of a stromatal localisation. NifH-tGFP visualization, however, showed punctuate signal accumulation around the chloroplasts, similar to what was observed in tobacco and consistent with the accumulation of an unproperly folded protein (Figures 14B and 32B). Co-expression of NifH and NifM resulted in diffuse eGFP and mCherry signals, both colocalizing with chlorophyll, confirming previous observations in tobacco (Section 7.2.2) and yeast (López-Torrejón et al., 2016) that NifM is required for the accumulation of soluble NifH. Furthermore, the co-localization of NifH and NifM proteins in chloroplasts indicated that their corresponding TPs, AtmRCATP and AtmBCCP $1_{\text {TP }}$ respectively, were functional in driving their import into the organelle, an important result, since their efficiency had only been previously tested using eGFP as cargo protein. 
(A)

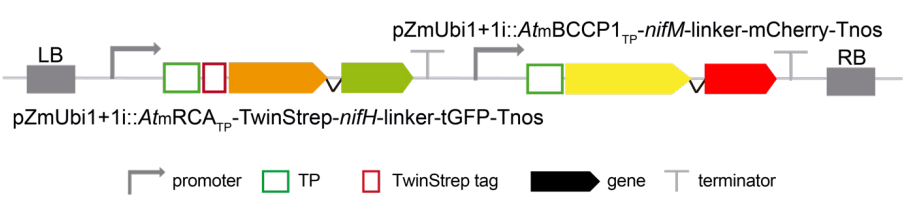

(B)
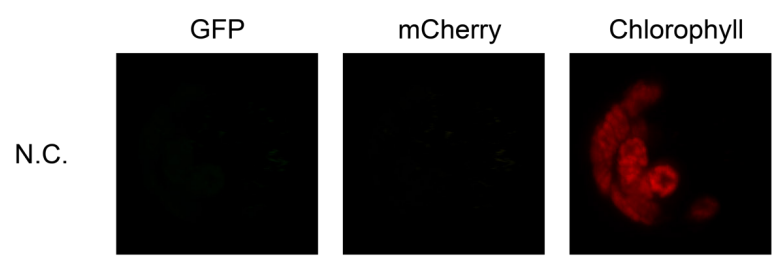

Merge
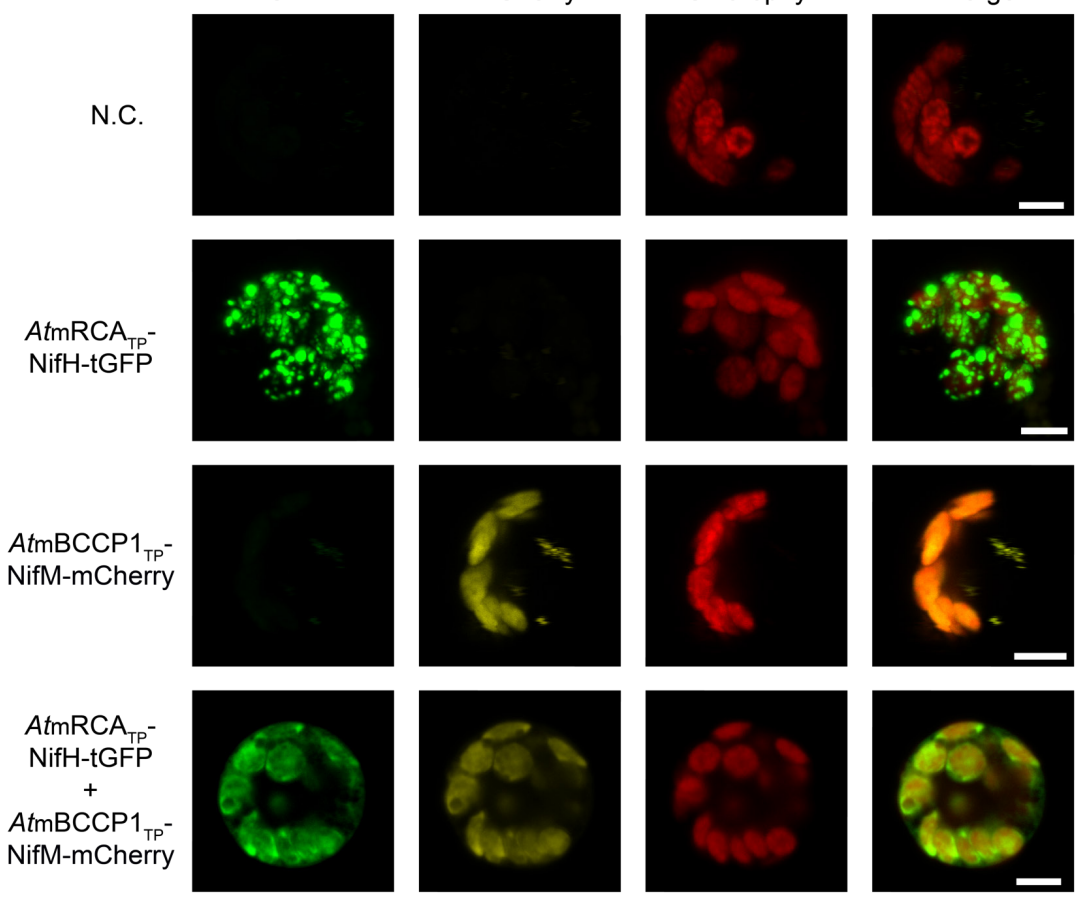

Figure 32. NifM remains necessary for the accumulation of soluble NifH in the stroma of rice cells. (A) Schematic representation of the construct used in O. sativa-transient expression assays leading to NifH and NifM co-localization. (B) Confocal microscopy images of co-expressed NifH and NifM proteins fused to tGFP and mCherry respectively. N.C. refers to non-transformed protoplasts. The four columns show the individual signals for tGFP (green), mCherry (yellow), and chlorophyll autofluorescence (red). On the right, the overlap of the three signals (merge). Note the co-localization of green and yellow signals in a diffuse pattern within the chloroplast.

\subsubsection{Stable transformation of rice embryos co-expressing nifH, nifM, nifU, and nifs}

Once optimal conditions had been established for Nif protein accumulation and import into the chloroplast stroma, multigenic constructs were assembled with the rice codonoptimized nif genes and fused to the selected TPs for stable transformation. Three different versions of vectors were generated, all containing chloroplast-targeted Streptagged nifH and non-tagged versions of nifM, nifU, and nifS together with the hpt gene for selection (Figure 33A). In the first version (v1) all nif genes expressions were driven by the pZmUbi1+1i promoter. In the second version (v2), the design tried to achieve the optimal nif gene expression ratio of 100:25:50:25 for nifH:nifM:nifS:nifU, mimicking their ratios in nitrogen-fixing cells of $A$. vinelandii (Poza-Carrion et al., 2014), by using 
different promoters to drive the expression of the different nif genes, i.e. pZmUbi1+1i for nifH, 2xp35s for nifM and nifU and pOsActin1 for nifS. In the third version (cyto), gene expression was driven by pZmUbi1+1i for all genes, and no TP was used so that all Nif proteins were targeted to the cytosol and used as size controls (Figure 33A).

Prior to embryo transformation, constructs described above were checked in transformation experiments of protoplasts obtained from cells of rice sheath and stem (Supplemental Figure 6). SDS-PAGE and immunoblot analysis of rice protoplasts extracts enabled the detection of a band corresponding to NifH, both with antibodies specific to the protein and against Strep-tag, after transformation with all three plasmids (v1, v2, and cyto) (Figure 33B). NifM was likewise detected with specific antibodies in all cases. However, in the case of NifU and NifS, no clear signal could be identified due to the same detection problems previously described.

(A)

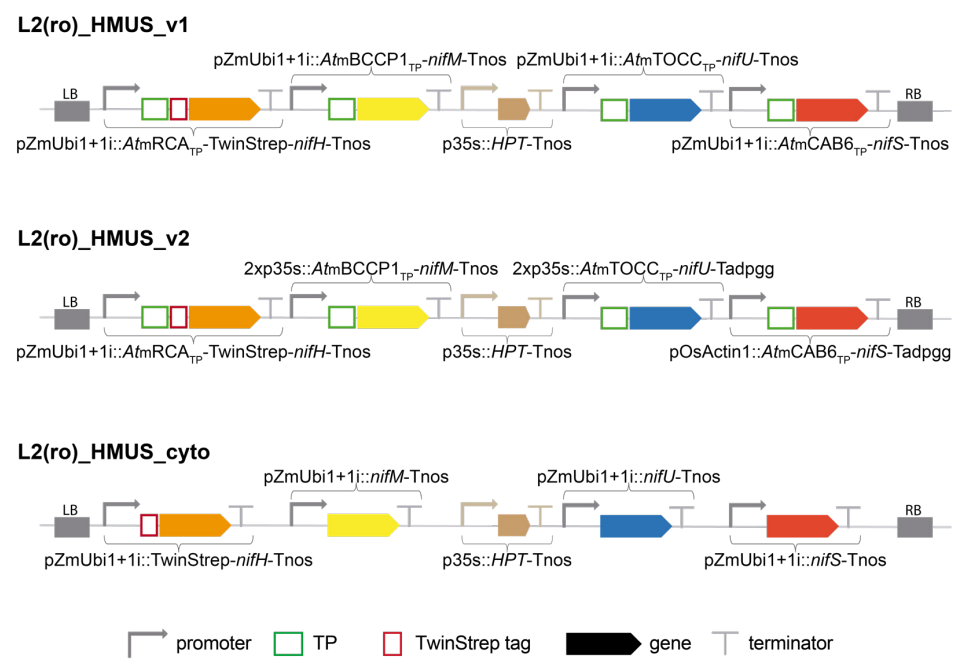

(B)
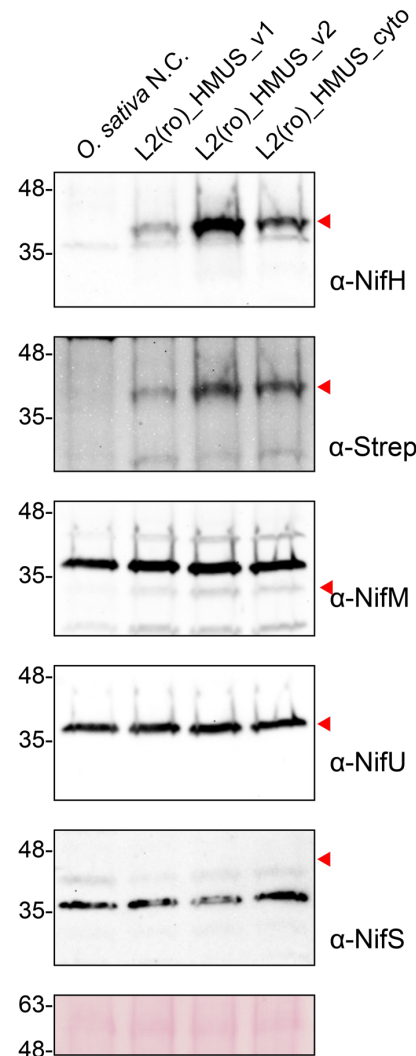

Figure 33. Multigenic constructs carrying nifH, nifM, nifU, and nifS for rice protoplast transformation.

(A) Schematic representation of the three multigenic constructs (v1, v2, and cyto) used in $O$. sativaprotoplast transient expression assays. (B) Western blot analysis of transformed protoplasts isolated from rice using antibodies targeting Strep-Tag, NifH, NifM, NifU, and NifS as indicated. N.C. refers to nontransformed protoplasts. The triangles in red mark the expected mature form of the protein. An intense $35 \mathrm{kDa}$ non-specific band can be observed when using $\alpha$-NifM, $\alpha$-NifU, and $\alpha$-NifS antibodies. Expected sizes are Twin-Strep NifH: 35.3 kDa, NifM: 32.3 kDa, NifU: 34.4 kDa, and NifS: 44.3 kDa. 
Transformation of rice embryos was carried out with v1, v2, and cyto plasmids (Figures 34A, C, and E, respectively). For each of the two first versions, 26 independent hygromycin resistant callus primordia were recovered out of 75 bombarded embryos. Regarding the cytosolic control, eight resistant lines were recovered. All 60 independent callus lines were screened for NifH expression by SDS-PAGE and immunoblot analysis with antibodies against NifH and Strep-tag, but no positive results were obtained in any case (Figures 34B, D, and F). Further studies will be conducted to determine the reasons preventing Nif proteins accumulation.

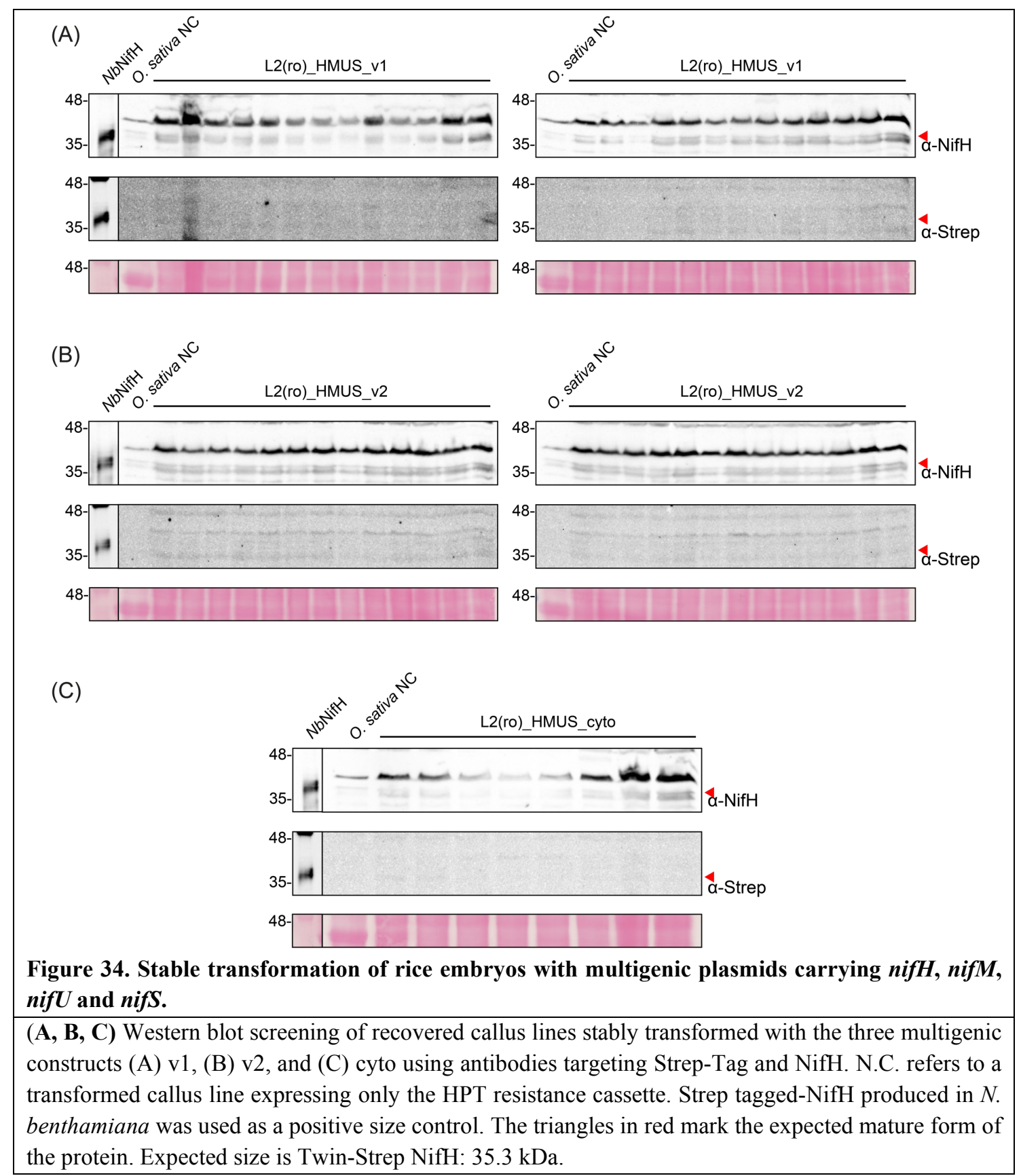




\subsection{Discussion}

Transient expression experiments provided proof of concept of tobacco chloroplasts being able to host active Fe protein at the end of the dark period (Section 7.2.3). However, the goal of ubiquitous expression of Nif proteins throughout plant tissues raises the question of whether chloroplast TPs could drive the import of fused cargo proteins into other plastid types, such as proplastids or leucoplasts. The latter ones are a promising location to host active nitrogenase in crops, as the cultivation procedure of rice plants involves waterlogged environments devoid of oxygen. Therefore, we embarked on the optimization of nif genes for their expression in rice and the characterization of previously validated minimal TPs in order to study their tissue and species specificity.

\subsubsection{Optimization of $\mathrm{NifH}$ biosynthetic pathway in rice plastids}

The use of nifH and nif $M$ genes synthetically designed for maximum expression in rice led to higher protein accumulation than other optimized versions (Figure 19). The rice codon-optimized and original $A$. vinelandii sequences scored comparable though slightly lower result. Noteworthy, the nifM rice synthetic design, which lacked two exclusion motifs, allowed us to obtain protein levels significantly higher than those achieved by using simply a codon-optimized sequence (Figure 19D, Supplemental Table 11). This constitutes a proof of concept that the removal of deleterious motifs in the DNA sequence can aid the expression and accumulation of a heterologous protein.

Regarding codon optimization, Nif accumulation patterns followed the same trend as the CAI (Figure 19, Supplemental Table 11). Remarkably, higher GC and GC3 content correlated with better gene expression and protein accumulation (Supplemental Table 11). GC content in rice codon-optimized and synthetic-designed sequences was between $60-64 \%$ and GC3 above $80 \%$, much higher than those of the CDS of rice (GC $55.26 \%$ and GC3 61.61\%). A similar correlation has been previously observed in maize, where using codon biasing strategies that increased GC and GC3 content above the average codon usage resulted in higher protein accumulation (Sidorenko et al., 2017).

Detection of NifU and NifS was not possible by immunoblot due to the presence of a pattern of unspecific bands or a lack of signal, respectively, while eGFP controls were detected (Figure 20). Plasmid construction mistakes or frameshift mutations were ruled out, as the standard pieces leading to NifU and NifS accumulation were successfully tested in other experiments and Sanger sequencing was performed in all constructions (Figures 9 and 19). Future efforts should be aimed at solving this technical problem. Possible alternatives include the cleaning polyclonal antibodies against a WT rice extract to eliminate non-specific binding, the use of specific antibodies against a fused protein 
tag, or the analysis of soluble extracts by targeted proteomics, a high-sensitivity, quantitative technique.

The observed compartmentalization of NifH in the absence of NifM is similar to what was observed in mesophyll cells from agroinfiltrated leaves of $N$. benthamiana (Figure 15), suggesting an incorrect maturation of the NifH protein when expressed in the absence of NifM and confirming its requirement for the correct maturation of $\mathrm{NifH}$ also in rice cells (Figure 32B).

\subsubsection{Arabidopsis TPs as tools for plastid import}

Earlier studies have identified preprotein specificities of import (Chu et al., 2020; Chu and Li, 2018; Fitzpatrick and Keegstra, 2001; Jarvis and López-Juez, 2013). However, our limited knowledge on this subject still makes a difficult task trying to infer pre-protein recognition and translocation efficiencies from the simple analysis of TPs' primary sequence and TP behaviour needs to be confirmed experimentally.

Apart from a general enrichment in alanine, serine, and uncharged amino acid residues, no consensus sequence motifs have been identified among TPs (Ivey et al., 2000; Zybailov et al., 2008). The TP sequences used here to direct the nuclear-encoded eGFP marker protein into plastids exhibit these characteristics, i.e. a prevalence in serine, alanine, and uncharged residues (cysteine, threonine, tyrosine, asparagine, and glutamine) compared to that of the mature protein (Figure 21A and Supplemental Figure 9).

Two motifs were identified in the TP of Arabidopsis RuBisCO small subunit that are responsible for its Toc159-dependent import pathway into chloroplasts (Lee et al., 2009). The first one, (DITSITSNGG), could not be found among the TPs in study here (Figure 21A). The second motif, consisting of a group of serine residues (SS), was present in AtmCAB6тP, AtmTOCC ${ }_{\mathrm{TP}}, A t \mathrm{mBCCP} 1_{\mathrm{TP}}, A t \mathrm{cGLTB} 2_{\mathrm{TP}}$, and rice $O s \mathrm{RBS} 1_{\mathrm{TP}}$ TPs. On the other hand, Vojta et al. (2004) proposed that positively charged amino acid residues at the C-terminus of TPs (positions -8 and -1 from the cleavage site) were involved in atToc34 recognition and import. This seems to be the case for the five Arabidopsis TPs studied here since they present positively charged residues around the fore-mentioned positions (Figure 21A). Contrary, OsRBS1 TP TP only presents two positively charged residues at -2 and -4 .

Our experimental results support that AtmCAB6тP, AtmRCAтP, and AtmBCCP1 $1_{\mathrm{TP}}$ TPs follow the atToc159-dependent import mechanism specific for photosynthetic proteins, which would explain their higher import efficiency into chloroplasts (Figure 24B). However, they must also be able to interact with the translocon machinery that is 
expressed constitutively across all tissues (mainly atToc132, atToc120, and atToc34) to be efficiently imported into leucoplasts (Figure 25C). The case of $A t \mathrm{mBCCP} 1_{\mathrm{TP}} \mathrm{TP}$ is interesting as it presents a high import efficiency into both chloroplasts and leucoplasts. This behaviour could be explained as BCCP1 is involved in fatty acid biosynthesis, which primarily takes place in chloroplasts of green tissues and plastids of non-photosynthetic tissues, from acetyl CoA, an abundant direct product of photosynthesis (Supplemental Figure 5). Moreover, expression and protein accumulation of BCCP1 between leaf and root tissues exhibit a lesser difference when compared with other TPs related proteins (Figure 21B). For AtmTOCC ${ }_{\text {TP }}$ and $A t c G L T B 2$ TP TPs, which show a similar degree of import rate in both plastid types (Figure 24B and 25C), our results support a preferential interaction with the TOC family members uniformly expressed across shoot and root.

The case of rice $O s \mathrm{RBS} 1_{\mathrm{TP}} \mathrm{TP}$ is surprising, showing only the ability to mediate import to plastids in rice (Figure 24B, 25C, and 27B). This would suggest some differences between the TIC/TOC systems of the two species analysed that avoid its recognition by Arabidopsis members. This idea is supported by some previous observations that point to a differential behaviour of TPs depending on the species they were tested. Pea CAB TP was shown to mediate preferential import of proteins into chloroplasts (Wan et al., 1996), while RCA TP conferred no such specificity, exhibiting comparable efficiencies in the import of nuclear-encoded proteins in chloroplasts and leucoplasts (Wan et al., 1995, 1996). As we have shown in this work, in Arabidopsis, the set of TPs are more effective in their capacity to import eGFP into chloroplasts, although eGFP import into leucoplasts was still quite efficient (Figure 24B). On the other hand, all Arabidopsis TPs performed very efficiently in rice chloroplasts (Figure 27B), and AtmCAB6TP, AtmRCATP, AtmTOCC ${ }_{\mathrm{TP}}$, and $A t \mathrm{mBCCP} 1_{\mathrm{TP}}$ abolished completely plastid type specificity in terms of their capacity of protein import (Figures 28C, 30A, and 31A). It seems that all the Arabidopsis TPs in study here lose their plastid type import specificity, which is prevalent in their native species-environment when expressed in rice cells. Moreover, OsRBS1 $1_{\mathrm{TP}}$ TP was completely unable to mediate plastid import in Arabidopsis, suggesting that the mechanisms regulating the import of nuclear-encoded proteins into plastids may not be highly conserved and appear to vary between species.

In general, protoplast transient expression experiments resulted in lower import efficiencies than those observed in plant stable expression. This could be explained by high protein expression being able to saturate the translocon machinery and leading to partial import. Thus, caution should be taken when TPs are characterized using only protoplasts, onion, or BY-2 cells experiments where these artificial effects are possible. 
Our results show low protein accumulation in transgenic plant roots (Figure 31) although the promoter used has been reported to drive higher expression in rice roots relative to leaves (Green et al., 2002). Whether this is due to lower protein production or degradation before plastid import remains to be studied. In addition, we note that all lines recovered after transformation with the AtmTOCC ${ }_{\mathrm{TP}}-\mathrm{eGFP}$ construct showed lower eGFP expression and protein accumulation. Future studies should be aimed at understanding if this can be avoided by any strategy such as specific codon usage optimization for rice.

Furthermore, the correct import of specific Nif proteins carried out by AtmRCATP and AtmBCCP1 $1_{\mathrm{TP}}$ validate their use to drive $\mathrm{NifH}$ and NifM into the chloroplasts of rice (Figures 32 and 33).

\subsubsection{Efforts in the generation of nif expressing rice lines}

Use of the v1 construct aimed to achieve high levels of protein accumulation by using a strong constitutive promoter, i.e. pZmUbil, to drive the expression of all nif genes (Figures 33, 34A, and 34B). Contrary to dicotyledonous species, the repeated use of the same promoter in multi-gene combinatorial experiments has not been related to any issues of homology-dependent gene silencing in rice or maize (Kohli et al., 2006). However, no signal for any Nif protein could be observed and the involvement of gene silencing remains to be determined in further studies.

The design of the v2 construct was an attempt to tailor expression using the few wellestablished constitutive promoters in rice (Figures 33, 34C, and 34D) (McElroy and Brettell, 1994). Recent work has characterized a set of standardized promoters and terminators for engineering legumes and cereals (Feike et al., 2019), which could help refine the engineering of nitrogenase biosynthesis even further. The fact that this strategy where the promoter driving nifH is unique supports the hypothesis that the problem inhibiting nif gene expression is not uniquely due to homology-driven silencing.

Whereas the multigenic constructs rendered NifH and NifM accumulation in rice protoplast transient expression experiments (Figure 33B), the attempt at generating stably transformed rice lines identified further barriers associated with the transformation procedure, as no lines accumulated detectable NifH in immunoblot assays (Figures 34B, D, and F). Further studies will be aimed at deciphering where the main bottleneck for Nif protein accumulation in rice resides. The analysis of the inserted DNA integrity and copy number determination of nif transgenes within the host genome will determine if the transformation method was appropriate. Additionally, mRNA Northern blots or RealTime quantitative PCR analysis will be used to analyse whether the lines positive for 
correct and complete insertions do express the integrated transgenes or not, in order to determine if the main problem resides at transgene expression (Bai et al., 2016; Sudhakar et al., 1998). Preliminary experiments were already done in some of the above-mentioned lines to assess if the problem could be derived from protein degradation due to the presence of oxygen in rice chloroplasts. This does not seem the case, although this line of investigation should also be continued before discarding this possibility. The data obtained from all these experiments will shed some light on the constraints we are facing and hopefully point a way to resume engineering of $\mathrm{NifH}$ biosynthesis in rice. 



\section{Discussion}

Plant synthetic biology seeks to engineer novel traits into plants in a predictable and understandable manner and plays a fundamental role in facing current challenges of climate change and world population rise. One major aspect of these challenges is the nitrogen availability to crops, which is a limiting factor of crop yield so much so that the use of synthetic nitrogen fertilizers is prevalent in developed countries to increase crop yield. The generation of nitrogen-fixing crops is one of the SynBio projects that could lead to a new Green Revolution and have a global impact on agriculture and environment preservation. The idea of generating plants that do not need nitrogen fertilizers is far from being new (Hardy and Havelka, 1975). Many groups have worked in the transfer of the genes encoding for the nitrogenase enzyme to eukaryotic hosts, however, most attempts failed in getting active protein (Burén et al., 2020; Burén and Rubio, 2018).

The nitrogenase Fe protein has been the protein of choice to assay the suitability of subcellular compartments to acquire and maintain its activity, given that it is highly oxygen-sensitive and its maturation steps are simpler than those of the MoFe protein. Within this context, this thesis has focused on testing the ability of plant plastids to host active nitrogenase $\mathrm{Fe}$ protein, for that we engaged in the optimization of prokaryotic nif genes for their expression in higher plants and the import of Nif proteins into their plastids.

\section{Azotobacter nif gene optimization for expression in higher plants}

We researched and established a set of parameters intended to increase nif gene expression and translation in plants, specifically, tobacco and rice, and with the aid of CAD tools, generated nif synthetic gene variants that resulted in enhanced accumulation of Nif proteins.

Transient expression assays in $N$. benthamiana proved that nifH, $M, U$, and $S$ genes synthetically designed to maximize expression in tobacco led to a much higher protein accumulation than the yeast-optimized ones, although those were theoretically also optimum for recombinant protein production in the tobacco system regarding codon optimization parameters. These results show that the extra-factors considered in our synthetic design beyond codon optimization had a determinant role in stimulating Nif protein production and stabilizing it.

Our work has been essential to enable the study of NifH functionality within plant cells. For a long time, plant engineering strategies seeking to achieve high levels of expression 
of transgenes were only based on the use of strong constitutive promoters. Dixon et al. (1997) proved that $K$. pneumoniae nifH and nif $M$ original sequences could be expressed in tobacco using the cauliflower mosaic virus $35 \mathrm{~S}$ promoter. They showed that genes were transcribed at relatively high levels in the plants. However, Western blotting experiments showed very little Nif protein accumulation, below the limits of detection for an activity assay. Thus, the question regarding the functionality of $\mathrm{NifH}$ in plant cells remained unanswered for more than 20 years.

A different strategy was used by Ivleva et al. (2016), using a transplastomic approach and benefiting from the prokaryotic nature of the organelle that assured efficient transcription and translation of original prokaryotic nif sequences. In this case, transplastomic plants carrying the original $A$. vinelandii nifH and nifM sequences rendered sufficient levels of protein to detect very low Fe protein activity in enriched extracts, but it was required to grow plants in an oxygen-depleted atmosphere. Since direct plastid genome transformation remains a challenge for most crops, this approach is not readily transferrable to cereals, and for those, our optimization pipeline showed great promise.

We followed a similar strategy as with $N$. benthamiana and generated nif synthetic gene variants optimized for $O$. sativa using monocot-specific parameters. Detection of NifU and NifS was not possible due to unspecific binding of their antibodies to several proteins within the rice extracts. However, nifH and nifM could be detected, and it was observed that the synthetic design led to a comparable protein accumulation to the one obtained using the rice codon-optimized and original $A$. vinelandii sequences, but much higher than the one obtained when using the yeast-optimized ones, as predicted by CAI values. Remarkably, a tight correlation between sequences with high GC and GC3 content values and higher protein accumulation was observed for both nifH and nif $M$ genes consistent with data previously reported (Sidorenko et al., 2017). The exclusion of deleterious motifs in the sequence of nif genes showed a tendency to improve protein accumulation, confirming our previous observations in tobacco.

Overall, our results show that adapting the codon usage frequencies of a foreign sequence to that of your host organism is not always enough to guarantee high gene expression and protein accumulation, and more complex strategies can be included in synthetic biology approaches in which obtaining high protein levels is a challenge.

\section{Optimization of protein import into plastids}

Import of nuclear-encoded proteins into plastids is an important bottleneck in fundamental plant research and many applications of plant biotechnology. The characterization of a set of TPs that facilitate recombinant protein import into different 
plastid types and that work with high efficiency in both model plants and crops increases the portfolio of standard parts available for plant SynBio and can be very useful for plant engineering projects. Even more in many staple crops including food security crops such as rice or maize, which are not amenable to direct plastid transformation.

The limited knowledge on the motifs responsible for TPs functionality impedes the prediction of their behaviour when carrying recombinant cargo proteins and their functionality needs to be confirmed experimentally. We have characterized a set of minimal transit peptides and analysed their behaviour in the import of eGFP and specific Nif proteins into the plastids of tobacco and rice, as a means to studying their modularity and orthogonality.

In $N$. benthamiana, we tested a library of 12 TPs fused to each of our four Nif proteins of interest. Most of the TPs previously described in the literature span past the SPP cleavage site and thus, leave scar amino acid residues in the N-terminus of the imported protein after their cleavage, which could affect cargo protein activity and folding. Thus, we also tested minimal versions of all 12 TPs, which included the TP coding sequence up until the predicted cleavage site plus the following alanine. Overall, the use of a minimal TP resulted in a higher mobility band compared to the complete version. However, in some cases, the minimal TP seemed to lose its ability to import the Nif protein into the chloroplast, possibly due to the removal of a motif that was needed for its recognition by the import machinery (Li and Teng, 2013).

It is noteworthy, but not surprising, that only two minimal TPs AtmGLTB2TP and AtmBCCP1 $1_{\text {TP }}$ performed properly for all four Nif proteins. It has been previously reported that tertiary structures of the cargo protein next to the TP sequence affect its function (Shen et al., 2017). Also, several reports have noted that a portion of the mature protein, usually an unfolded region following the $\mathrm{TP}$, is in some cases required for proper import (Bionda et al., 2010). Therefore, experiments performed with carrier proteins as GFP o mCherry are useful to characterize a TP but do not accurately predict its behaviour when fused to the specific cargo protein of interest.

In an attempt to characterize useful TPs, we selected four of the best-performing minimal TPs in tobacco $\left(A t m R C A_{\mathrm{TP}}, A t \mathrm{mBCCP} 1_{\mathrm{TP}}\right.$, AtmTOCC ${ }_{\mathrm{TP}}$, and AtmCAB6 ${ }_{\mathrm{TP}}$ ) together with one commonly used from the target host $\left(O s \mathrm{RBS} 1_{\mathrm{TP}}\right)$, and, to test how promiscuous TPs are in different tissues, we incorporated a TP coming from a gene highly expressed in roots $(A t c G L T B 2 \mathrm{TP})$. This library was tested for eGFP import efficiency in leaves and roots cells of Arabidopsis and rice. In Arabidopsis, we observed that the set of TPs selected is generally more effective in their capacity to import eGFP into chloroplasts 
compared to leucoplasts, with the noteworthy exception of $O s \mathrm{RBS} 1_{\mathrm{TP}}$, which was unable to mediate plastid import, and $A t \mathrm{mBCCP} 1_{\mathrm{TP}}$, which presented a high import efficiency into both plastid types. All Arabidopsis TPs performed very efficiently in eGFP import into rice chloroplasts from protoplast, losing their species import specificity, whereas $O s \mathrm{RBS} 1_{\mathrm{TP}}$, although functional, was significantly less efficient. Interestingly, $O s \mathrm{RBS} 1_{\mathrm{TP}}$ was the only TP presenting two positively charged residues, involved in atToc34 recognition and import, at different positions than the ones described by Vojta et al. (2004), possibly interfering in its function in Arabidopsis; whereas its low efficiency in rice may be due to the import competition with the endogenous $\mathrm{RuBisCO}$ protein. We further confirmed that all four minimal TPs were functional and highly efficient in driving eGFP into proplastids, chloroplast, and leucoplast of stably transformed rice plants, confirming the results obtained in the transient system. Additionally, we proved that $A t \mathrm{mRCA}$ TP and $A t \mathrm{mBCCP} 1_{\mathrm{TP}}$ minimal TPs were effective in targeting NifH and NifM to chloroplasts of rice cells, validating their use as engineering tools for future projects.

NifM, a protein with similarity to prolyl isomerases, is essential for NifH activity in its original host (Howard et al., 1986) and when expressed in both mitochondria and cytoplasm of yeast (López-Torrejón et al., 2016). Our results in tobacco and rice cells clearly show that, when expressed alone, NifH was correctly imported into plastids, but accumulated in a punctuated pattern. Simultaneously expressing a soluble marker of the stroma traced the compartmentalisation of NifH to a point after their import, possibly following a pathway for degradation of improperly folded proteins. Yet, when coexpressed with NifM, soluble stroma NifH accumulation was obtained, showing that the maturase is also a requirement for achieving a functional Fe protein in plant cells.

\section{Engineering nitrogenase Fe protein biosynthesis in plastids}

The production of Nif proteins that accumulate to high levels in N. benthamiana leaf cells and that are correctly imported into chloroplasts facilitated the recovery of active nitrogenase Fe protein, with no doubt, a major accomplishment. Although oxygenic photosynthesis might be providing an unfavourable environment for nitrogenase $\mathrm{Fe}$ protein maturation during the day, our results show that at least at night, leaf cell chloroplasts constitute an environment with low enough oxygen for nitrogenase $\mathrm{Fe}$ protein accumulation in active form. The activity obtained is less than a half of the one obtained from yeast mitochondria (López-Torrejón et al., 2016) and about a sixth of the one from $A$. vinelandii, so there is still room for improvement in the engineering but constitutes a considerable achievement. Previously, only very low NifH activity had been detected in transplastomic plants expressing NifH and NifM, and only after incubation of the plants in an oxygen-depleted atmosphere (Ivleva et al., 2016). Our results constitute 
the first definitive proof of concept that engineering Fe protein biosynthesis in plastids of higher plants is an achievable strategy. However, future efforts should be directed at finetuning $\mathrm{NifH}$ maturation and protection of its $\mathrm{Fe}-\mathrm{S}$ clusters from oxidative damage, perhaps by the engineering of a temporal separation of nitrogen fixation and photosynthesis (Curatti and Rubio, 2014) or by co-expressing the FeSII protein that could bind NifH for cluster protection (Moshiri et al., 1995; Schlesier et al., 2016).

Nitrogenase activity relies on its metal clusters, which require significant amounts of iron and molybdenum. The [Fe-S] clusters need to be properly assembled and delivered into nitrogenase components, a task carried out in diazotrophs by NifS, which mobilizes the $\mathrm{S}$ required, and NifU, where cluster assembly takes place for its subsequently delivery.

Previously, NifU and NifS were found not to be necessary for Fe protein activity in the mitochondria of yeast, suggesting that mitochondrial $[\mathrm{Fe}-\mathrm{S}]$ cluster assembly machinery can substitute at least some of the functions from these Nif proteins (López-Torrejón et al., 2016), though NifU and NifS were in fact required for NifB activity in yeast mitochondria (Burén et al., 2019). However, our results show that, in plant plastids, NifH lacks any activity when only co-expressed with NifM, suggesting that the chloroplast [Fe$\mathrm{S}$ ] cluster machinery cannot, at least in transient assays, efficiently replace the function of NifU and NifS. Nevertheless, plants are rich in [Fe-S] clusters due to their requirement in the photosynthesis machinery, and its assembly pathways, primarily located in mitochondria and chloroplasts, are similar to those found in bacteria (Balk and Pilon, 2011). Given the importance of [Fe-S] clusters in plants, it is conceivable that [Fe-S] carrier proteins in plastids play redundant roles and under certain conditions transfer clusters promiscuously, a possibility that may be exploited in the future to eliminate the need for nif $U$ and nifS in the engineering of an active nitrogenase (Couturier et al., 2013).

\section{Perspectives of nitrogen-fixing plants}

SynBio tools can be of great help in the optimization of the expression of foreign genes, protein accumulation, and other biological processes like organelle transport. The use of transient expression assays together with modular cloning techniques and fast-growing model plants have proved extremely useful in our optimization pipeline. We were able to perform several iterations of the DBTL cycle in a short time, enabling the optimization of each of the different elements that constituted an engineering challenge, in order to prove that chloroplasts are indeed a suitable location to host active Fe protein, a goal not achieved for more than 20 years.

SynBio allowed us to decouple the complex problem of Fe protein biosynthesis in plants into smaller and simpler steps, that were addressed individually. After the optimization 
of each specific transcriptional unit for each nif gene, it was fairly straightforward to pile them all up to test their co-expression and $\mathrm{NifH}$ functionality in transient expression assays within tobacco. But our objective is to transfer these results to stably transformed crops.

As a further step in this direction, we used rice protoplast transient expression assays. Although this seems useful to fine-tune host species-specific preferences, there are still significant differences between transiently and stably transformed plants. Protoplast assays showed lower chloroplast import efficiencies than those observed in stably transformed rice plants and, while NifH presence could be detected in rice protoplasts, no stably transformed lines accumulated detectable NifH when checked on immunoblot assays. Though it is technically possible to engineer rice plants expressing four or even more transgenes (Zhu et al., 2008), failure to express transgenic proteins in plants has frequently been observed. A number of different causes have been linked to those failures, including positional effects of the chromatin environment due to the random nature of the integration (Butaye et al., 2005) or gene silencing (Vaucheret and Fagard, 2001). When using biolistic transformation, severe genome rearrangements have been described in the genome of host plants (Liu et al., 2019), and transgene aberrant structures are commonly generated (Fu et al., 2000). Therefore, the use of other transformation methods for rice, such as Agrobacterium-mediated T-DNA transfer, could be an approach worth considering (Hiei and Komari, 2008).

Despite the success in obtaining NifH activity in tobacco, the engineering of the different nif modules necessary to achieve an active nitrogenase enzyme in plants is lagging behind, but SynBio approaches can help tackle these problems. NifB from A. vinelandii, a key enzyme in the nitrogenase pathway, was found insoluble in mitochondria of yeast and tobacco, but bioinformatic mining in databases found a relative from Methanothermobacter thermautotrophicus that accumulated as soluble protein and was functional (Burén et al., 2019). Moreover, NifD from A. vinelandii and $K$. oxytoca suffered from $\mathrm{N}$-terminal degradation in mitochondria of yeast and tobacco due to a recognition motif for a mitochondrial endoprotease. The identification of the motif and engineering of amino acid mutations resulted in variants that were resistant to degradation, as well as retained function solving a bottleneck to the assembly of active MoFe protein (Allen et al., 2020; Xiang et al., 2020).

The development of tools that facilitate crop engineering is of the uttermost importance for plant SynBio. Many efforts in the characterization of genetic modules, gene optimization and protein targeting in crops are currently underway (Baysal et al., 2020; 
Feike et al., 2019). In the future, it will be interesting to study whether the tools developed in this thesis can work with other Nif proteins required for nitrogenase biosynthesis. In any case, the pipeline described for the optimization of each element used in their engineering provides an example of how complex projects can be achieved. In the future, we will focus on the transition from model to staple crops like rice and maize, where our efforts can lead to the final goal of generating nitrogen-fixing cereals. 



\section{Conclusions}

1. We established a set of species-specific parameters to increase gene expression and protein accumulation and generated nif synthetic gene variants that resulted in enhanced accumulation of Nif proteins.

2. The experimental validation of transit peptides is critical. In Nicotiana benthamiana, in some cases, the minimal transit peptides seemed to lose their ability to import the cargo protein into chloroplasts and their behaviour was influenced by different Nif cargo proteins.

3. Four minimal transit peptides, namely $A t m R C A_{\mathrm{TP}}, A t \mathrm{mBCCP} 1_{\mathrm{TP}}, A t \mathrm{mTOCC} \mathrm{TP}_{\mathrm{TP}}$ and $A t \mathrm{mCAB} 6_{\mathrm{TP}}$, proved that they correctly and accurately drive the import of NifH, NifM, NifU and NifS respectively, into the chloroplast stroma of Nicotiana benthamiana cells.

4. Soluble stroma NifH accumulation required of NifM co-expression, confirming that the maturase is a requirement for achieving soluble Fe protein in both plant cells assayed, tobacco and rice.

5. We recovered active nitrogenase $\mathrm{Fe}$ protein from chloroplasts of tobacco mesophyll cells, providing the proof of concept that, at least at night, this location constitutes a suitable environment for engineering Fe protein biosynthesis, which is dependent on NifU and NifS for cluster delivery.

6. We observed that AtmRCATP, AtmTOCC ${ }_{\mathrm{TP}}$, and $A t \mathrm{mCAB} 6_{\mathrm{TP}}$ transit peptides were more effective in their capacity to import eGFP into chloroplasts compared to leucoplasts of Arabidopsis thaliana, while AtmBCCP1 $1_{\mathrm{TP}}$ presented a high import efficiency into both plastid types.

7. AtmRCA ${ }_{\mathrm{TP}}, A t \mathrm{mBCCP} 1_{\mathrm{TP}}, A t \mathrm{mTOCC} \mathrm{TP}_{\mathrm{TP}}$, and $A t \mathrm{mCAB} 6_{\mathrm{TP}}$ transit peptides were functional and highly efficient in driving eGFP into proplastids and chloroplast from Oryza sativa, losing their species import specificity. AtmRCATP, AtmTOCC $\mathrm{TP}$, and AtmCAB6TP further mediated eGFP import efficiently into rice leucoplast.

8. We demonstrated that $A t m R C A_{T P}$ and $A t m B C C P 1_{\mathrm{TP}}$ were also effective in targeting NifH and NifM cargo proteins to the stroma of chloroplasts from Oryza sativa cells. 



\section{References}

Adem, M., Beyene, D., and Feyissa, T. (2017) Recent achievements obtained by chloroplast transformation. Plant Methods, 13, 30.

Allen, R.S., Gregg, C.M., Okada, S., Menon, A., Hussain, D., Gillespie, V., et al. (2020) Plant expression of NifD protein variants resistant to mitochondrial degradation. Proc. Natl. Acad. Sci., 117, 23165-23173.

Allen, R.S., Tilbrook, K., Warden, A.C., Campbell, P.C., Rolland, V., Singh, S.P., and Wood, C.C. (2017) Expression of 16 Nitrogenase Proteins within the Plant Mitochondrial Matrix. Front. Plant Sci., 8, 287.

Altpeter, F., Springer, N.M., Bartley, L.E., Blechl, A., Brutnell, T.P., Citovsky, V., et al. (2016) Advancing Crop Transformation in the Era of Genome Editing. Plant Cell, 28, tpc.00196.2016.

Andrianantoandro, E., Basu, S., Karig, D.K., and Weiss, R. (2006) Synthetic biology: new engineering rules for an emerging discipline. Mol. Syst. Biol., 2, 2006.0028.

Ausländer, S., Ausländer, D., and Fussenegger, M. (2017) Synthetic Biology-The Synthesis of Biology. Angew. Chemie Int. Ed., 56, 6396-6419.

Awika, J.M. (2011) Major Cereal Grains Production and Use around the World. In: ACS Symposium Series , pp. 1-13. American Chemical Society.

Baerenfaller, K., Grossmann, J., Grobei, M.A., Hull, R., Hirsch-Hoffmann, M., Yalovsky, S., et al. (2008) Genome-scale proteomics reveals Arabidopsis thaliana gene models and proteome dynamics. Science (80-. )., 320, 938-941.

Bai, C., Capell, T., Berman, J., Medina, V., Sandmann, G., Christou, P., and Zhu, C. (2016) Bottlenecks in carotenoid biosynthesis and accumulation in rice endosperm are influenced by the precursor-product balance. Plant Biotechnol. J., 14, 195-205.

Balk, J. and Pilon, M. (2011) Ancient and essential: the assembly of iron-sulfur clusters in plants. Trends Plant Sci., 16, 218-226.

Bally, J., Jung, H., Mortimer, C., Naim, F., Philips, J.G., Hellens, R., et al. (2018) The Rise and Rise of Nicotiana benthamiana: A Plant for All Reasons. Annu. Rev. Phytopathol., 56, 405-426.

Balsera, M., Soll, J., and Bölter, B. (2009) Protein import machineries in endosymbiotic organelles. Cell. Mol. Life Sci., 66, 1903-1923.

Barampuram, S. and Zhang, Z.J. (2011) Recent Advances in Plant Transformation. In: Methods in molecular biology (Clifton, N.J.), pp. 1-35. Humana Press, Totowa, NJ.

Batista-Silva, W., da Fonseca-Pereira, P., Martins, A.O., Zsögön, A., Nunes-Nesi, A., and Araújo, W.L. (2020) Engineering Improved Photosynthesis in the Era of Synthetic Biology. Plant Commun., 1, 100032.

Baysal, C., Pérez-González, A., Eseverri, Á., Jiang, X., Medina, V., Caro, E., et al. (2020) Recognition motifs rather than phylogenetic origin influence the ability of targeting peptides to import nuclear-encoded recombinant proteins into rice mitochondria. Transgenic Res., 29, 37-52.

Beatty, P.H. and Good, A.G. (2011) Future Prospects for Cereals That Fix Nitrogen. Science (80-. )., 333, 416-417.

Benchabane, M., Goulet, C., Rivard, D., Faye, L., Gomord, V., and Michaud, D. (2008) Preventing unintended proteolysis in plant protein biofactories. Plant Biotechnol. $J ., 6,633-648$.

Bernabé-Orts, J.M., Quijano-Rubio, A., Vazquez-Vilar, M., Mancheño-Bonillo, J., Moles-Casas, V., Selma, S., et al. (2020) A memory switch for plant synthetic biology based on the phage $\phi C 31$ integration system. Nucleic Acids Res., 48, 3379-3394. 
Bertani, G. (1951) Studies on lysogenesis. I. The mode of phage liberation by lysogenic Escherichia coli. J. Bacteriol., 62, 293-300.

Bionda, T., Tillmann, B., Simm, S., Beilstein, K., Ruprecht, M., and Schleiff, E. (2010) Chloroplast Import Signals: The Length Requirement for Translocation In Vitro and In Vivo. J. Mol. Biol., 402, 510-523.

Bishop, P.E. and Joerger, R.D. (1990) Genetics and Molecular Biology of Alternative Nitrogen Fixation Systems. Annu. Rev. Plant Physiol. Plant Mol. Biol., 41, 109-125.

Bloch, S.E., Ryu, M.-H., Ozaydin, B., and Broglie, R. (2020) Harnessing atmospheric nitrogen for cereal crop production. Curr. Opin. Biotechnol., 62, 181-188.

Bock, R. (2015) Engineering Plastid Genomes: Methods, Tools, and Applications in Basic Research and Biotechnology. Annu. Rev. Plant Biol., 66, 211-241.

Boehm, C.R. and Bock, R. (2019) Recent Advances and Current Challenges in Synthetic Biology of the Plastid Genetic System and Metabolism. Plant Physiol., 179, 794802.

Boehm, C.R., Pollak, B., Purswani, N., Patron, N., and Haseloff, J. (2017) Synthetic Botany. Cold Spring Harb. Perspect. Biol., 9, a023887.

Boeing, P., Ozdemir, T., and Barnes, C. (2016) Design Tools for Synthetic Biology. In: Synthetic Biology Handbook, pp. 259-280. CRC Press.

Borlaug, N.E. (1972) Nobel Lecture, December 11, 1970. From Nobel Lectures, Peace 1951-1970, Frederick W. Haberman Ed.

Bouis, H.E. and Saltzman, A. (2017) Improving nutrition through biofortification: A review of evidence from HarvestPlus, 2003 through 2016. Glob. Food Sec., 12, 4958.

Van den Broeck, G., Timko, M.P., Kausch, A.P., Cashmore, A.R., Van Montagu, M., and Herrera-Estrella, L. (1985) Targeting of a foreign protein to chloroplasts by fusion to the transit peptide from the small subunit of ribulose 1,5-bisphosphate carboxylase. Nature, 313, 358-363.

Bruce, B.D. (2000) Chloroplast transit peptides: Structure, function and evolution. Trends Cell Biol., 10, 440-447.

Bruce, B.D. (2001) The paradox of plastid transit peptides: Conservation of function despite divergence in primary structure. Biochim. Biophys. Acta - Mol. Cell Res., 1541, 2-21.

Burén, S., Jiang, X., López-Torrejón, G., Echavarri-Erasun, C., and Rubio, L.M. (2017) Purification and In Vitro Activity of Mitochondria Targeted Nitrogenase Cofactor Maturase NifB. Front. Plant Sci., 8, 1567.

Burén, S., Jiménez-Vicente, E., Echavarri-Erasun, C., and Rubio, L.M. (2020) Biosynthesis of Nitrogenase Cofactors. Chem. Rev., 120, 4921-4968.

Burén, S., Pratt, K., Jiang, X., Guo, Y., Jimenez-Vicente, E., Echavarri-Erasun, C., et al. (2019) Biosynthesis of the nitrogenase active-site cofactor precursor NifB-co in Saccharomyces cerevisiae. Proc. Natl. Acad. Sci., 116, 25078-25086.

Burén, S. and Rubio, L.M. (2018) State of the art in eukaryotic nitrogenase engineering. FEMS Microbiol. Lett., 365.

Burén, S., Young, E.M., Sweeny, E.A., Lopez-Torrejón, G., Veldhuizen, M., Voigt, C.A., and Rubio, L.M. (2017) Formation of Nitrogenase NifDK Tetramers in the Mitochondria of Saccharomyces cerevisiae. ACS Synth. Biol., 6, 1043-1055.

Butaye, K.M.J., Cammue, B.P.A., Delauré, S.L., and De Bolle, M.F.C. (2005) Approaches to Minimize Variation of Transgene Expression in Plants. Mol. Breed., 16, 79-91.

Cai, Y.-M., Kallam, K., Tidd, H., Gendarini, G., Salzman, A., and Patron, N.J. (2020) Rational design of minimal synthetic promoters for plants. Nucleic Acids Res., $\mathbf{4 8 ,}$ 
$11845-11856$.

Canfield, D.E., Glazer, A.N., and Falkowski, P.G. (2010) The Evolution and Future of Earth's Nitrogen Cycle. Science (80-. )., 330, 192-196.

Carswell, S. and Alwine, J.C. (1989) Efficiency of utilization of the simian virus 40 late polyadenylation site: effects of upstream sequences. Mol. Cell. Biol., 9, 4248-4258.

Carvalho, J. de F.C., Madgwick, P.J., Powers, S.J., Keys, A.J., Lea, P.J., and Parry, M.A.J. (2011) An engineered pathway for glyoxylate metabolism in tobacco plants aimed to avoid the release of ammonia in photorespiration. BMC Biotechnol., 11, 111.

Cela, J., Chang, C., and Munné-Bosch, S. (2011) Accumulation of $\gamma$-rather than $\alpha$ tocopherol alters ethylene signaling gene expression in the vte4 Mutant of Arabidopsis thaliana. Plant Cell Physiol., 52, 1389-1400.

Chen, X., Smith, M.D., Fitzpatrick, L., and Schnell, D.J. (2002) In vivo analysis of the role of atTic20 in protein import into chloroplasts. Plant Cell, 14, 641-654.

Cheng, Q., Day, A., Dowson-Day, M., Shen, G.-F., and Dixon, R. (2005) The Klebsiella pneumoniae nitrogenase Fe protein gene (nifH) functionally substitutes for the chlL gene in Chlamydomonas reinhardtii. Biochem. Biophys. Res. Commun., 329, 966975.

Chin, J.X., Chung, B.K.-S., and Lee, D.-Y. (2014) Codon Optimization OnLine (COOL): a web-based multi-objective optimization platform for synthetic gene design. Bioinformatics, 30, 2210-2212.

Chotewutmontri, P., Holbrook, K., and Bruce, B.D. (2017) Plastid Protein Targeting. In: International Review of Cell and Molecular Biology, pp. 227-294. Elsevier Inc.

Chou, T.-C. and Moyle, R.L. (2014) Synthetic versions of firefly luciferase and Renilla luciferase reporter genes that resist transgene silencing in sugarcane. BMC Plant Biol., 14, 92.

Christou, P., Ford, T.L., and Kofron, M. (1991) Production of Transgenic Rice (Oryza Sativa L.) Plants from Agronomically Important Indica and Japonica Varieties via Electric Discharge Particle Acceleration of Exogenous DNA into Immature Zygotic Embryos. Bio/Technology, 9, 957-962.

Chu, C.-C. and Li, H. min (2018) Developmental regulation of protein import into plastids. Photosynth. Res., 138, 327-334.

Chu, C.-C., Swamy, K., and Li, H. (2020) Tissue-Specific Regulation of Plastid Protein Import via Transit-Peptide Motifs. Plant Cell, 32, 1204-1217.

Chua, N.H. and Schmidt, G.W. (1978) Post-translational transport into intact chloroplasts of a precursor to the small subunit of ribulose-1,5-bisphosphate carboxylase. Proc. Natl. Acad. Sci. U. S. A., 75, 6110-6114.

Chung, B. and Lee, D.-Y. (2012) Computational codon optimization of synthetic gene for protein expression. BMC Syst. Biol., 6, 134.

Clarke, J.L. and Daniell, H. (2011) Plastid biotechnology for crop production: present status and future perspectives. Plant Mol. Biol., 76, 211-220.

Clough, S.J. and Bent, A.F. (1998) Floral dip: A simplified method for Agrobacteriummediated transformation of Arabidopsis thaliana. Plant J., 16, 735-743.

Collin, V.C., Eymery, F., Genty, B., Rey, P., and Havaux, M. (2008) Vitamin E is essential for the tolerance of Arabidopsis thaliana to metal-induced oxidative stress. Plant, Cell Environ., 31, 244-257.

Compant, S., Samad, A., Faist, H., and Sessitsch, A. (2019) A review on the plant microbiome: Ecology, functions, and emerging trends in microbial application. J. Adv. Res., 19, 29-37.

Coruzzi, G., Broglie, R., Edwards, C., and Chua, N.H. (1984) Tissue-specific and light- 
regulated expression of a pea nuclear gene encoding the small subunit of ribulose1,5-bisphosphate carboxylase. EMBO J., 3, 1671-1679.

Couturier, J., Touraine, B., Briat, J.-F., Gaymard, F., and Rouhier, N. (2013) The ironsulfur cluster assembly machineries in plants: current knowledge and open questions. Front. Plant Sci., 4, 259.

Csorba, T., Kontra, L., and Burgyán, J. (2015) viral silencing suppressors: Tools forged to fine-tune host-pathogen coexistence. Virology, 479-480, 85-103.

Cui, K. and Shoemaker, S.P. (2018) Public perception of genetically-modified (GM) food: A Nationwide Chinese Consumer Study. npj Sci. Food, 2, 10.

Curatti, L., Hernandez, J.A., Igarashi, R.Y., Soboh, B., Zhao, D., and Rubio, L.M. (2007) In vitro synthesis of the iron molybdenum cofactor of nitrogenase from iron, sulfur, molybdenum, and homocitrate using purified proteins. Proc. Natl. Acad. Sci., 104, 17626-17631.

Curatti, L. and Rubio, L.M. (2014) Challenges to develop nitrogen-fixing cereals by direct nif-gene transfer. Plant Sci., 225, 130-137.

Daher, Z., Recorbet, G., Valot, B., Robert, F., Balliau, T., Potin, S., et al. (2010) Proteomic analysis of Medicago truncatula root plastids. Proteomics, 10, 21232137.

Damm, B., Schmidt, R., and Willmitzer, L. (1989) Efficient transformation of Arabidopsis thaliana using direct gene transfer to protoplasts. Mol. Gen. Genet. $M G G, 217,6-12$.

Dixon, R., Cheng, Q., Shen, G.F., Day, A., and Dowson-Day, M. (1997) Nif gene transfer and expression in chloroplasts: Prospects and problems. Plant Soil, 194, 193-203.

Dixon, R.A. and Postgate, J.R. (1972) Genetic Transfer of Nitrogen Fixation from Klebsiella pneumoniae to Escherichia coli. Nature, 237, 102-103.

Dobberstein, B., Blobel, G., and Chua, N.H. (1977) In vitro synthesis and processing of a putative precursor for the small subunit of ribulose 1,5 bisphosphate carboxylase of Chlamydomonas reinhardtii. Proc. Natl. Acad. Sci. U. S. A., 74, 1082-1085.

Dong, O.X. and Ronald, P.C. (2019) Genetic Engineering for Disease Resistance in Plants: Recent Progress and Future Perspectives. Plant Physiol., 180, 26-38.

Dutta, S., Teresinski, H.J., and Smith, M.D. (2014) A split-ubiquitin yeast two-hybrid screen to examine the substrate specificity of atToc159 and atToc132, two Arabidopsis chloroplast preprotein import receptors. PLoS One, 9, e95026.

Egelkrout, E., Rajan, V., and Howard, J.A. (2012) Overproduction of recombinant proteins in plants. Plant Sci., 184, 83-101.

Emami, S., Yee, M., and Dinneny, J.R. (2013) A robust family of Golden Gate Agrobacterium vectors for plant synthetic biology. Front. Plant Sci., 4, 339.

Emanuelsson, O., Nielsen, H., and Heijne, G. Von (1999) ChloroP, a neural networkbased method for predicting chloroplast transit peptides and their cleavage sites. Protein Sci., 8, 978-984.

Endy, D. (2005) Foundations for engineering biology. Nature, 438, 449-453.

Engler, C., Gruetzner, R., Kandzia, R., and Marillonnet, S. (2009) Golden Gate Shuffling: A One-Pot DNA Shuffling Method Based on Type IIs Restriction Enzymes. PLoS One, 4, e5553.

Engler, C., Youles, M., Gruetzner, R., Ehnert, T.-M., Werner, S., Jones, J.D.G., et al. (2014) A Golden Gate Modular Cloning Toolbox for Plants. ACS Synth. Biol., 3, 839-843.

Erisman, J.W., Galloway, J.N., Dise, N.B., Sutton, M.A., Bleeker, A., Grizzetti, B., et al. (2015) Nitrogen: too much of a vital resource: Science Brief. WWF Netherlands.

Erisman, J.W. and Sutton, M.A. (2008) Reduced nitrogen in ecology and the 
environment: Special issue of the ESF-FWF Conference in partnership with LFUI, October 2006. Environ. Pollut., 154, 357-358.

Erisman, J.W., Sutton, M.A., Galloway, J., Klimont, Z., and Winiwarter, W. (2008) How a century of ammonia synthesis changed the world. Nat. Geosci., 1, 636-639.

Eseverri, Á., López-Torrejón, G., Jiang, X., Burén, S., Rubio, L.M., and Caro, E. (2020) Use of synthetic biology tools to optimize the production of active nitrogenase Fe protein in chloroplasts of tobacco leaf cells. Plant Biotechnol. J., 18, 1882-1896.

Falcón, L.I., Magallón, S., and Castillo, A. (2010) Dating the cyanobacterial ancestor of the chloroplast. ISME J., 4, 777-783.

Feike, D., Korolev, A. V., Soumpourou, E., Murakami, E., Reid, D., Breakspear, A., et al. (2019) Characterizing standard genetic parts and establishing common principles for engineering legume and cereal roots. Plant Biotechnol. J., 17, 22342245.

de Felippes, F., McHale, M., Doran, R.L., Roden, S., Eamens, A.L., Finnegan, E.J., and Waterhouse, P.M. (2020) The key role of terminators on the expression and posttranscriptional gene silencing of transgenes. Plant J., 104, 96-112.

Feng, P.C.C., Marianne, M., and Stanislaw, F. (2010) Chloroplast transit peptides for efficient targeting of DMO and uses thereof.

Fitzpatrick, L.M. and Keegstra, K. (2001) A method for isolating a high yield of Arabidopsis chloroplasts capable of efficient import of precursor proteins. Plant J., 27, 59-65.

Flavell, R. (2009) Role of Model Plant Species. In: Methods in Molecular Biology, pp. 1-18. Methods Mol Biol.

Foyer, C.H., Noctor, G., and Hodges, M. (2011) Respiration and nitrogen assimilation: targeting mitochondria-associated metabolism as a means to enhance nitrogen use efficiency. J. Exp. Bot., 62, 1467-1482.

Fredens, J., Wang, K., de la Torre, D., Funke, L.F.H., Robertson, W.E., Christova, Y., et al. (2019) Total synthesis of Escherichia coli with a recoded genome. Nature, 569, 514-518.

Fu, W., Jack, R.F., Morgan, T.V., Dean, D.R., and Johnson, M.K. (1994) nifU Gene Product from Azotobacter vinelandii Is a Homodimer That Contains Two Identical [2Fe-2S] Clusters. Biochemistry, 33, 13455-13463.

Fu, X., Kohli, A., Twyman, R.M., and Christou, P. (2000) Alternative silencing effects involve distinct types of non-spreading cytosine methylation at a three-gene, singlecopy transgenic locus in rice. Mol. Gen. Genet., 263, 106-118.

Galanie, S., Thodey, K., Trenchard, I.J., Filsinger Interrante, M., and Smolke, C.D. (2015) Complete biosynthesis of opioids in yeast. Science (80-. )., 349, 1095-1100.

Gallegos, J.E. and Rose, A.B. (2015) The enduring mystery of intron-mediated enhancement. Plant Sci., 237, 8-15.

Gavini, N., Tungtur, S., and Pulakat, L. (2006) Peptidyl-Prolyl cis/trans IsomeraseIndependent Functional NifH Mutant of Azotobacter vinelandii. J. Bacteriol., 188, 6020-6025.

Georgiadis, M., Komiya, H., Chakrabarti, P., Woo, D., Kornuc, J., and Rees, D. (1992) Crystallographic structure of the nitrogenase iron protein from Azotobacter vinelandii. Science (80-. )., 257, 1653-1659.

Gilchrist, M. and Benjamin, N. (2017) From Atmospheric Nitrogen to Bioactive Nitrogen Oxides. In: Nitrite and Nitrate in Human Health and Disease, pp. 11-19. Cham: Springer International Publishing.

Gleba, Y., Klimyuk, V., and Marillonnet, S. (2005) Magnifection - A new platform for expressing recombinant vaccines in plants. In: Vaccine, pp. 2042-2048. Elsevier 
BV.

Gleizer, S., Ben-Nissan, R., Bar-On, Y.M., Antonovsky, N., Noor, E., Zohar, Y., et al. (2019) Conversion of Escherichia coli to Generate All Biomass Carbon from CO2. Cell, 179, 1255-1263.e12.

González-García, M.-P., Bustillo-Avendaño, E., Sanchez-Corrionero, A., del Pozo, J.C., and Moreno-Risueno, M.A. (2020) Fluorescence-Activated Cell Sorting Using the D-Root Device and Optimization for Scarce and/or Non-Accessible Root Cell Populations. Plants, 9, 499.

Good, A. (2018) Toward nitrogen-fixing plants. Science (80-. )., 359, 869-870.

Gould, N., Hendy, O., and Papamichail, D. (2014) Computational Tools and Algorithms for Designing Customized Synthetic Genes. Front. Bioeng. Biotechnol., 2, 41.

Green, J., Vain, P., Fearnehough, M.T., Worland, B., Snape, J.W., and Atkinson, H.J. (2002) Analysis of the expression patterns of the Arabidopsis thaliana tubulin-1 and Zea mays ubiquitin-1 promoters in rice plants in association with nematode infection. Physiol. Mol. Plant Pathol., 60, 197-205.

Grobbelaar, N., Huang, T.C., Lin, H.Y., and Chow, T.J. (1986) Dinitrogen-fixing endogenous rhythm in Synechococcus RF-1. FEMS Microbiol. Lett., 37, 173-177.

Guidance for risk assessment of food and feed from genetically modified plants (2011) EFSA J., 9, 2150.

Gustafsson, C., Minshull, J., Govindarajan, S., Ness, J., Villalobos, A., and Welch, M. (2012) Engineering genes for predictable protein expression. Protein Expr. Purif., 83, 37-46.

Gutiérrez, MacIntosh, and Green (1999) Current perspectives on mRNA stability in plants: multiple levels and mechanisms of control. Trends Plant Sci., 4, 429-438.

Haffani, Y.Z., Overney, S., Yelle, S., Bellemare, G., and Belzile, F.J. (2000) Premature polyadenylation contributes to the poor expression of the Bacillus thuringiensis cry3Cal gene in transgenic potato plants. Mol. Gen. Genet. MGG, 264, 82-88.

Halbleib, C.M. and Ludden, P.W. (2000) Regulation of Biological Nitrogen Fixation. J. Nutr., 130, 1081-1084.

Hanahan, D., Jessee, J., and Bloom, F.R. (1991) Plasmid transformation of Escherichia coli and other bacteria. Methods Enzymol., 204, 63-113.

Hardy, R.W.F. and Havelka, U.D. (1975) Nitrogen Fixation Research: A Key to World Food? Science (80-. )., 188, 633-643.

Hiei, Y., Ishida, Y., and Komari, T. (2014) Progress of cereal transformation technology mediated by Agrobacterium tumefaciens. Front. Plant Sci., 5, 1-11.

Hiei, Y. and Komari, T. (2008) Agrobacterium-mediated transformation of rice using immature embryos or calli induced from mature seed. Nat. Protoc., 3, 824-834.

Holland, C.K. and Jez, J.M. (2018) Arabidopsis: the original plant chassis organism. Plant Cell Rep., 37, 1359-1366.

Hoppmann, V., Fiore, S.D. i., Zimmermann, S., Emans, N., Rademacher, T., Fischer, R., and Schillberg, S. (2002) The potato granule bound starch synthase chloroplast transit peptide directs recombinant proteins to plastids. J. Plant Physiol., 159, 1061-1067.

Howard, K.S., McLean, P.A., Hansen, F.B., Lemley, P. V, Koblan, K.S., and OrmeJohnson, W.H. (1986) Klebsiella pneumoniae nifM gene product is required for stabilization and activation of nitrogenase iron protein in Escherichia coli. J. Biol. Chem., 261, 772-8.

Huff, J. (2015) The Airyscan detector from ZEISS: confocal imaging with improved signal-to-noise ratio and super-resolution. Nat. Methods, 12, i-ii.

Hutchison, C.A., Chuang, R.-Y., Noskov, V.N., Assad-Garcia, N., Deerinck, T.J., 
Ellisman, M.H., et al. (2016) Design and synthesis of a minimal bacterial genome. Science (80-. )., 351, aad6253-aad6253.

Ingelbrecht, I.L., Herman, L.M., Dekeyser, R.A., Van Montagu, M.C., and Depicker, A.G. (1989) Different 3' end regions strongly influence the level of gene expression in plant cells. Plant Cell, 1, 671-80.

Inoue, H., Rounds, C., and Schnell, D.J. (2010) The molecular basis for distinct pathways for protein import into arabidopsis chloroplasts. Plant Cell, 22, 1947-1960.

ISAAA (2020) ISAAA GM Approval Database. [online] www.isaaa.org. Available www.isaaa.org/gmapprovaldatabase [Accessed Oct. 2020].

Ivanova, Y., Smith, M.D., Chen, K., and Schnell, D.J. (2004) Members of the Toc159 import receptor family represent distinct pathways for protein targeting to plastids. Mol. Biol. Cell, 15, 3379-3392.

Ivey, R.A., Subramanian, C., and Bruce, B.D. (2000) Identification of a Hsp70 recognition domain within the Rubisco small subunit transit peptide. Plant Physiol., 122, 1289-1299.

Ivleva, N.B., Groat, J., Staub, J.M., and Stephens, M. (2016) Expression of Active Subunit of Nitrogenase via Integration into Plant Organelle Genome. PLoS One, 11, e0160951.

Jackson, M.A., Sternes, P.R., Mudge, S.R., Graham, M.W., and Birch, R.G. (2014) Design rules for efficient transgene expression in plants. Plant Biotechnol. J., 12, 925-933.

Jacobson, M.R., Cash, V.L., Weiss, M.C., Laird, N.F., Newton, W.E., and Dean, D.R. (1989) Biochemical and genetic analysis of the nifUSVWZM cluster from Azotobacter vinelandii. MGG Mol. Gen. Genet., 219, 49-57.

Jarvis, P. (2008) Targeting of nucleus-encoded proteins to chloroplasts in plants. New Phytol., 179, 257-285.

Jarvis, P. and López-Juez, E. (2013) Biogenesis and homeostasis of chloroplasts and other plastids. Nat. Rev. Mol. Cell Biol., 14, 787-802.

Ji, G., Zheng, J., Shen, Y., Wu, X., Jiang, R., Lin, Y., et al. (2007) Predictive modeling of plant messenger RNA polyadenylation sites. BMC Bioinformatics, $\mathbf{8}, 43$.

Jiang, X., Payá-Tormo, L., Coroian, D., García-Rubio, I., Castellanos-Rueda, R., Eseverri, Á., et al. (2020) Exploiting genetic diversity and gene synthesis to identify superior nitrogenase NifH protein variants to engineer N2-fixation in plants. Commun. Biol.

Kadkhodaei, S., Memari, H.R., Abbasiliasi, S., Rezaei, M.A., Movahedi, A., Shun, T.J., and Ariff, A. Bin (2016) Multiple overlap extension PCR (MOE-PCR): an effective technical shortcut to high throughput synthetic biology. RSC Adv., 6, 66682-66694.

Kavanagh, T.A., Jefferson, R.A., and Bevan, M.W. (1988) Targeting a foreign protein to chloroplasts using fusions to the transit peptide of a chlorophyll a/b protein. $M G G$ Mol. Gen. Genet., 215, 38-45.

Kearse, M., Moir, R., Wilson, A., Stones-Havas, S., Cheung, M., Sturrock, S., et al. (2012) Geneious Basic: An integrated and extendable desktop software platform for the organization and analysis of sequence data. Bioinformatics, 28, 1647-1649.

Kebeish, R., Niessen, M., Thiruveedhi, K., Bari, R., Hirsch, H.-J., Rosenkranz, R., et al. (2007) Chloroplastic photorespiratory bypass increases photosynthesis and biomass production in Arabidopsis thaliana. Nat. Biotechnol., 25, 593-599.

Kennedy, I.R. and Tchan, Y.-T. (1992) Biological nitrogen fixation in non-leguminous field crops: Recent advances. Plant Soil, 141, 93-118.

Kiani, S., Mohamed, B.B., Shehzad, K., Jamal, A., Shahid, M.N., Shahid, A.A., and Husnain, T. (2013) Chloroplast-targeted expression of recombinant crystal-protein 
gene in cotton: An unconventional combat with resistant pests. J. Biotechnol., 166, 88-96.

Kim, E.H., Suh, S.C., Park, B.S., Shin, K.S., Kweon, S.J., Han, E.J., et al. (2009) Chloroplast-targeted expression of synthetic crylAc in transgenic rice as an alternative strategy for increased pest protection. Planta, 230, 397-405.

Kim, J. and Rees, D.C. (1992) Crystallographic structure and functional implications of the nitrogenase molybdenum-iron protein from azotobacter vinelandii. Nature, $\mathbf{3 6 0}$, 553-60.

Kim, S., Lee, D.-S., Choi, I.S., Ahn, S.-J., Kim, Y.-H., and Bae, H.-J. (2010) Arabidopsis thaliana Rubisco small subunit transit peptide increases the accumulation of Thermotoga maritima endoglucanase Cel5A in chloroplasts of transgenic tobacco plants. Transgenic Res., 19, 489-497.

Klee, H.J., Muskopf, Y.M., and Gasser, C.S. (1987) Cloning of an Arabidopsis thaliana gene encoding 5-enolpyruvylshikimate-3-phosphate synthase: sequence analysis and manipulation to obtain glyphosate-tolerant plants. MGG Mol. Gen. Genet., 210, 437-442.

Kleffmann, T., Russenberger, D., Von Zychlinski, A., Christopher, W., Sjölander, K., Gruissem, W., and Baginsky, S. (2004) The Arabidopsis thaliana chloroplast proteome reveals pathway abundance and novel protein functions. Curr. Biol., 14, 354-362.

Kleine, T., Maier, U.G., and Leister, D. (2009) DNA Transfer from Organelles to the Nucleus: The Idiosyncratic Genetics of Endosymbiosis. Annu. Rev. Plant Biol., 60, $115-138$.

Klinkenberg, J. (2014) Extraction of Chloroplast Proteins from Transiently Transformed Nicotiana benthamiana Leaves. BIO-PROTOCOL, 4.

Knight, T. (2003) Idempotent Vector Design for Standard Assembly of Biobricks. MIT Libr., 1-11.

Kohli, A., González-Melendi, P., Abranches, R., Capell, T., Stoger, E., and Christou, P. (2006) The quest to understand the basis and mechanisms that control expression of introduced transgenes in crop plants. Plant Signal. Behav., 1, 185-195.

Koncz, C. and Schell, J. (1986) The promoter of TL-DNA gene 5 controls the tissuespecific expression of chimaeric genes carried by a novel type of Agrobacterium binary vector. Mol. Gen. Genet. MGG, 204, 383-396.

Kovacheva, S., Bédard, J., Patel, R., Dudley, P., Twell, D., Ríos, G., et al. (2005) In vivo studies on the roles of Tic110, Tic40 and Hsp93 during chloroplast protein import. Plant J., 41, 412-428.

Kozak, M. (1986) Influences of $m R N A$ secondary structure on initiation by eukaryotic ribosomes. Proc. Natl. Acad. Sci., 83, 2850-2854.

Ladha, J.K. and Reddy, P.M. (1995) Extension of nitrogen fixation to rice - Necessity and possibilities. GeoJournal, 35, 363-372.

Laemmli, U.K. (1970) Cleavage of structural proteins during the assembly of the head of bacteriophage T4. Nature, 227, 680-5.

Le, Q.H., Wright, S., Yu, Z., and Bureau, T. (2000) Transposon diversity in Arabidopsis thaliana. Proc. Natl. Acad. Sci. U. S. A., 97, 7376-81.

Lee, D.W. and Hwang, I. (2011) Transient expression and analysis of chloroplast proteins in arabidopsis protoplasts. Methods Mol. Biol., 774, 59-71.

Lee, D.W., Kim, J.K., Lee, S., Choi, S., Kim, S., and Hwang, I. (2008) Arabidopsis Nuclear-Encoded Plastid Transit Peptides Contain Multiple Sequence Subgroups with Distinctive Chloroplast-Targeting Sequence Motifs. Plant Cell, 20, 1603-1622.

Lee, D.W., Lee, S., Oh, Y.J., and Hwang, I. (2009) Multiple sequence motifs in the 
rubisco small subunit transit peptide independently contribute to Toc159-dependent import of proteins into chloroplasts. Plant Physiol., 151, 129-141.

Li, H. min and Teng, Y.S. (2013) Transit peptide design and plastid import regulation. Trends Plant Sci., 18, 360-366.

Li, X. (2011) Infiltration of Nicotiana benthamiana Protocol for Transient Expression via Agrobacterium. BIO-PROTOCOL, 1.

Lill, R. and Mühlenhoff, U. (2008) Maturation of Iron-Sulfur Proteins in Eukaryotes: Mechanisms, Connected Processes, and Diseases. Annu. Rev. Biochem., 77, 669700 .

Lin, M.T., Occhialini, A., Andralojc, P.J., Parry, M.A.J., and Hanson, M.R. (2014) A faster Rubisco with potential to increase photosynthesis in crops. Nature, 513, 547550.

Liu, D., Liberton, M., Yu, J., Pakrasi, H.B., and Bhattacharyya-Pakrasi, M. (2018) Engineering Nitrogen Fixation Activity in an Oxygenic Phototroph. MBio, 9, 1-12.

Liu, J., Nannas, N.J., Fu, F.-F., Shi, J., Aspinwall, B., Parrott, W.A., and Dawe, R.K. (2019) Genome-Scale Sequence Disruption Following Biolistic Transformation in Rice and Maize. Plant Cell, 31, 368-383.

Liu, W. and Stewart, C.N. (2015) Plant synthetic biology. Trends Plant Sci., 20, 309317.

Liu, W., Yuan, J.S., and Stewart Jr, C.N. (2013) Advanced genetic tools for plant biotechnology. Nat. Rev. Genet., 14, 781-793.

López-Torrejón, G., Jiménez-Vicente, E., Buesa, J.M., Hernandez, J.A., Verma, H.K., and Rubio, L.M. (2016) Expression of a functional oxygen-labile nitrogenase component in the mitochondrial matrix of aerobically grown yeast. Nat. Commun., 7, 11426.

Luo, X., Reiter, M.A., D’Espaux, L., Wong, J., Denby, C.M., Lechner, A., et al. (2019) Complete biosynthesis of cannabinoids and their unnatural analogues in yeast. Nature, 567, 123-126.

Maier, A., Fahnenstich, H., von Caemmerer, S., Engqvist, M.K.M., Weber, A.P.M., Flügge, U.-I., and Maurino, V.G. (2012) Transgenic Introduction of a Glycolate Oxidative Cycle into A. thaliana Chloroplasts Leads to Growth Improvement. Front. Plant Sci., 3, 38.

Makrides, S.C. (1996) Strategies for achieving high-level expression of genes in Escherichia coli. Microbiol. Rev., 60, 512-538.

Martin, M.H. and Marschner, H. (1988) The Mineral Nutrition of Higher Plants. J. Ecol., 76, 1250 .

Mathew, L.G., Maloney, B., Takeda, N., and Mason, H.S. (2011) Spurious polyadenylation of Norovirus Narita 104 capsid protein $m R N A$ in transgenic plants. Plant Mol. Biol., 75, 263-275.

Mazur, B.J. and Chui, C.F. (1985) Sequence of a genomic DNA clone for the small subunit of ribulose bis-phosphate carboxylase-oxygenase from tobacco. Nucleic Acids Res., 13, 2373-2386.

McElroy, D. and Brettell, R.I.S. (1994) Foreign gene expression in transgenic cereals. Trends Biotechnol., 12, 62-68.

Meng, F. and Ellis, T. (2020) The second decade of synthetic biology: 2010-2020. Nat. Commun., 11, 5174.

Merrick, M. and Dixon, R. (1984) Why don't plants fix nitrogen? Trends Biotechnol., 2, $162-166$.

Mitsui, A., Kumazawa, S., Takahashi, A., Ikemoto, H., Cao, S., and Arai, T. (1986) Strategy by which nitrogen-fixing unicellular cyanobacteria grow 
photoautotrophically. Nature, 323, 720-722.

Mortimer, J.C. (2019) Plant synthetic biology could drive a revolution in biofuels and medicine. Exp. Biol. Med., 244, 323-331.

Moshiri, F., Crouse, B.R., Johnson, M.K., and Maier, R.J. (1995) The "nitrogenaseprotective" FeSII protein of Azotobacter vinelandii: overexpression, characterization, and crystallization. Biochemistry, 34, 12973-12982.

Mueller, N.D., Gerber, J.S., Johnston, M., Ray, D.K., Ramankutty, N., and Foley, J.A. (2012) Closing yield gaps through nutrient and water management. Nature, 490, 254-257.

Murashige, T. and Skoog, F. (1962) A Revised Medium for Rapid Growth and Bio Assays with Tobacco Tissue Cultures. Physiol. Plant., 15, 473-497.

Mus, F., Crook, M.B., Garcia, K., Garcia Costas, A., Geddes, B.A., Kouri, E.D., et al. (2016) Symbiotic Nitrogen Fixation and the Challenges to Its Extension to Nonlegumes. Appl. Environ. Microbiol., 82, 3698-3710.

Muthamilselvan, T., Kim, J.S., Cheong, G., and Hwang, I. (2019) Production of recombinant proteins through sequestration in chloroplasts: a strategy based on nuclear transformation and post-translational protein import. Plant Cell Rep., $\mathbf{3 8 ,}$ 825-833.

Naim, F., Nakasugi, K., Crowhurst, R.N., Hilario, E., Zwart, A.B., Hellens, R.P., et al. (2012) Advanced engineering of lipid metabolism in Nicotiana benthamiana using a draft genome and the V2 viral silencing-suppressor protein. PLoS One, 7, e52717.

Nakamura, Y., Gojobori, T., and Ikemura, T. (2000) Codon usage tabulated from international DNA sequence databases: status for the year 2000. Nucleic Acids Res., 28, 292.

Nakata, M.T., Sato, M., Wakazaki, M., Sato, N., Kojima, K., Sekine, A., et al. (2018) Plastid translation is essential for lateral root stem cell patterning in Arabidopsis thaliana. Biol. Open, 7.

Naseri, Z., Khezri, G., Davarpanah, S.J., and Ofoghi, H. (2019) Virus-based vectors: A new approach for the production of recombinant proteins. J. Appl. Biotechnol. Reports, 6, 6-14.

Nicolia, A., Manzo, A., Veronesi, F., and Rosellini, D. (2014) An overview of the last 10 years of genetically engineered crop safety research An overview of the last 10 years of genetically engineered crop safety research. Crit Rev Biotechnol, 34, 1549-7801.

Oldroyd, G.E. and Dixon, R. (2014) Biotechnological solutions to the nitrogen problem. Curr. Opin. Biotechnol., 26, 19-24.

Ortiz-Marquez, J.C.F., Do Nascimento, M., Zehr, J.P., and Curatti, L. (2013) Genetic engineering of multispecies microbial cell factories as an alternative for bioenergy production. Trends Biotechnol., 31, 521-529.

Otegui, M.S. (2018) Vacuolar degradation of chloroplast components: Autophagy and beyond. J. Exp. Bot., 69, 741-750.

Paddon, C.J., Westfall, P.J., Pitera, D.J., Benjamin, K., Fisher, K., McPhee, D., et al. (2013) High-level semi-synthetic production of the potent antimalarial artemisinin. Nature, 496, 528-532.

Page, M.T., Parry, M.A.J., and Carmo-Silva, E. (2019) A high-throughput transient expression system for rice. Plant. Cell Environ., 42, 2057-2064.

Pan, Y., Chen, R., Zhu, L., Wang, H., Huang, D., and Lang, Z. (2016) Utilizing modified ubil introns to enhance exogenous gene expression in maize (Zea mays L.) and rice (Oryza sativa L.). J. Integr. Agric., 15, 1716-1726.

Pardo, J. and Caro, E. (2017) ePARTS: An easy way to store and access your MoClo construct information in plant biotechnology projects. J. Plant Biotechnol. 
Microbiol., 1, 1-7.

Patron, N.J. (2020) Beyond natural: synthetic expansions of botanical form and function. New Phytol., 227, 295-310.

Patron, N.J., Orzaez, D., Marillonnet, S., Warzecha, H., Matthewman, C., Youles, M., et al. (2015) Standards for plant synthetic biology: a common syntax for exchange of DNA parts. New Phytol., 208, 13-9.

Peeters, N. and Small, I. (2001) Dual targeting to mitochondria and chloroplasts. Biochim. Biophys. Acta - Mol. Cell Res., 1541, 54-63.

Pérez-González, A. and Caro, E. (2018) Effect of transcription terminator usage on the establishment of transgene transcriptional gene silencing. BMC Res. Notes, 11, 511.

Pérez-González, A., Eseverri, Á., and Caro, E. (2018) Characterization of plant genetic modifications using next-generation sequencing. In: Synthetic Biology: Omics Tools and Their Applications, pp. 249-259.

Pérez-González, A., Kniewel, R., Veldhuizen, M., Verma, H.K., Navarro-Rodríguez, M., Rubio, L.M., and Caro, E. (2017) Adaptation of the GoldenBraid modular cloning system and creation of a toolkit for the expression of heterologous proteins in yeast mitochondria. BMC Biotechnol., 17, 80.

Pouvreau, B., Vanhercke, T., and Singh, S. (2018) From plant metabolic engineering to plant synthetic biology: The evolution of the design/build/test/learn cycle. Plant Sci., 273, 3-12.

Poza-Carrion, C., Jimenez-Vicente, E., Navarro-Rodriguez, M., Echavarri-Erasun, C., and Rubio, L.M. (2014) Kinetics of nif Gene Expression in a Nitrogen-Fixing Bacterium. J. Bacteriol., 196, 595-603.

Purnick, P.E.M. and Weiss, R. (2009) The second wave of synthetic biology: from modules to systems. Nat. Rev. Mol. Cell Biol., 10, 410-422.

Que, Q., Wang, H.Y., English, J.J., and Jorgensen, R.A. (1997) The Frequency and Degree of Cosuppression by Sense Chalcone Synthase Transgenes Are Dependent on Transgene Promoter Strength and Are Reduced by Premature Nonsense Codons in the Transgene Coding Sequence. Plant Cell, 9, 1357-1368.

Richardson, S.M., Mitchell, L.A., Stracquadanio, G., Yang, K., Dymond, J.S., DiCarlo, J.E., et al. (2017) Design of a synthetic yeast genome. Science (80-. )., 355, 10401044.

Richly, E. and Leister, D. (2004) An improved prediction of chloroplast proteins reveals diversities and commonalities in the chloroplast proteomes of Arabidopsis and rice. Gene, 329, 11-16.

Richter, S. and Lamppa, G.K. (2002) Determinants for removal and degradation of transit peptides of chloroplast precursor proteins. J. Biol. Chem., 277, 4388843894.

Roberts, G.P., MacNeil, T., MacNeil, D., and Brill, W.J. (1978) Regulation and characterization of protein products coded by the nif (nitrogen fixation) genes of Klebsiella pneumoniae. J. Bacteriol., 136, 267-279.

Rogers, C. and Oldroyd, G.E.D. (2014) Synthetic biology approaches to engineering the nitrogen symbiosis in cereals. J. Exp. Bot., 65, 1939-1946.

Rose, A.B. (2008) Intron-Mediated Regulation of Gene Expression. In: Current Topics in Microbiology and Immunology, pp. 277-290. Springer, Berlin, Heidelberg.

Rose, A.B. (2004) The effect of intron location on intron-mediated enhancement of gene expression in Arabidopsis. Plant J., 40, 744-751.

Rosenblueth, M., Ormeño-Orrillo, E., López-López, A., Rogel, M.A., Reyes-Hernández, B.J., Martínez-Romero, J.C., et al. (2018) Nitrogen fixation in cereals. Front. Microbiol., 9, 1794. 
Rovner, A.J., Haimovich, A.D., Katz, S.R., Li, Z., Grome, M.W., Gassaway, B.M., et al. (2015) Recoded organisms engineered to depend on synthetic amino acids. Nature, 518, 89-93.

Ryu, M.-H., Zhang, J., Toth, T., Khokhani, D., Geddes, B.A., Mus, F., et al. (2020) Control of nitrogen fixation in bacteria that associate with cereals. Nat. Microbiol., 5, 314-330.

Sánchez-León, S., Gil-Humanes, J., Ozuna, C. V., Giménez, M.J., Sousa, C., Voytas, D.F., and Barro, F. (2018) Low-gluten, nontransgenic wheat engineered with CRISPR/Cas9. Plant Biotechnol. J., 16, 902-910.

Dos Santos, P.C., Smith, A.D., Frazzon, J., Cash, V.L., Johnson, M.K., and Dean, D.R. (2004) Iron-sulfur cluster assembly: NifU-directed activation of the nitrogenase Fe protein. J. Biol. Chem., 279, 19705-19711.

Sarrion-Perdigones, A., Falconi, E.E., Zandalinas, S.I., Juárez, P., Fernández-delCarmen, A., Granell, A., and Orzaez, D. (2011) GoldenBraid: An Iterative Cloning System for Standardized Assembly of Reusable Genetic Modules. PLoS One, 6, e21622.

Sarrion-Perdigones, A., Vazquez-Vilar, M., Palaci, J., Castelijns, B., Forment, J., Ziarsolo, P., et al. (2013) GoldenBraid 2.0: A Comprehensive DNA Assembly Framework for Plant Synthetic Biology. PLANT Physiol., 162, 1618-1631.

Scharff, L.B. and Bock, R. (2014) Synthetic biology in plastids. Plant J., 78, 783-798.

Schlesier, J., Rohde, M., Gerhardt, S., and Einsle, O. (2016) A Conformational Switch Triggers Nitrogenase Protection from Oxygen Damage by Shethna Protein II (FeSII). J. Am. Chem. Soc., 138, 239-247.

Schwille, P. (2011) Bottom-Up Synthetic Biology: Engineering in a Tinkerer's World. Science (80-. )., 333, 1252-1254.

Sechi, S. and Chait, B.T. (1998) Modification of cysteine residues by alkylation. A tool in peptide mapping and protein identification. Anal. Chem., 70, 5150-8.

Seefeldt, L.C., Hoffman, B.M., and Dean, D.R. (2009) Mechanism of Mo-Dependent Nitrogenase. Annu. Rev. Biochem., 78, 701-722.

Shaer, O., Valdes, C., Liu, S., Lu, K., Haddock, T.L., Bhatia, S., et al. (2013) MoClo planner: Interactive visualization for Modular Cloning bio-design. In: 2013 IEEE Symposium on Biological Data Visualization (BioVis), pp. 57-64. IEEE.

Shah, V.K. and Brill, W.J. (1973) Nitrogenase IV. Simple method of purification to homogeneity of nitrogenase components from Azotobacter vinelandii. Biochim. Biophys. Acta - Bioenerg., 305, 445-454.

Shah, V.K., Pate, J.L., and Brill, W.J. (1973) Protection of Nitrogenase in Azotobacter vinelandii. J. Bacteriol., 115, 15-17.

Shanmugaraj, B., I. Bulaon, C.J., and Phoolcharoen, W. (2020) Plant Molecular Farming: A Viable Platform for Recombinant Biopharmaceutical Production. Plants, 9, 842 .

Sharif, M.K., Butt, M.S., Anjum, F.M., and Khan, S.H. (2014) Rice Bran: A Novel Functional Ingredient. Crit. Rev. Food Sci. Nutr., 54, 807-816.

Sharp, P.M. and Li, W.-H. (1987) The codon adaptation index-a measure of directional synonymous codon usage bias, and its potential applications. Nucleic Acids Res., 15, 1281-1295.

Shen, B.-R., Wang, L.-M., Lin, X.-L., Yao, Z., Xu, H.-W., Zhu, C.-H., et al. (2019) Engineering a New Chloroplastic Photorespiratory Bypass to Increase Photosynthetic Efficiency and Productivity in Rice. Mol. Plant, 12, 199-214.

Shen, B.-R., Zhu, C.-H., Yao, Z., Cui, L.-L., Zhang, J.-J., Yang, C.-W., et al. (2017) An optimized transit peptide for effective targeting of diverse foreign proteins into 
chloroplasts in rice. Sci. Rep., 7, 46231.

Shen, Y., Ji, G., Haas, B.J., Wu, X., Zheng, J., Reese, G.J., and Li, Q.Q. (2008) Genome level analysis of rice $m R N A$ 3???-end processing signals and alternative polyadenylation. Nucleic Acids Res., 36, 3150-3161.

Shetty, R.P., Endy, D., and Knight, T.F. (2008) Engineering BioBrick vectors from BioBrick parts. J. Biol. Eng., $2,5$.

Shi, L.-X. and Theg, S.M. (2010) A Stromal Heat Shock Protein 70 System Functions in Protein Import into Chloroplasts in the Moss Physcomitrella patens. Plant Cell, 22, 205-220.

Siddique, M.A., Grossmann, J., Gruissem, W., and Baginsky, S. (2006) Proteome Analysis of Bell Pepper ( Capsicum annuum L.) Chromoplasts. Plant Cell Physiol., 47, 1663-1673.

Sidorenko, L. V., Lee, T., Woosley, A., Moskal, W.A., Bevan, S.A., Merlo, P.A.O., et al. (2017) GC-rich coding sequences reduce transposon-like, small RNA-mediated transgene silencing. Nat. Plants, 3, 875-884.

Slusarczyk, A.L., Lin, A., and Weiss, R. (2012) Foundations for the design and implementation of synthetic genetic circuits. Nat. Rev. Genet., 13, 406-420.

Smanski, M.J., Bhatia, S., Zhao, D., Park, Y., B A Woodruff, L., Giannoukos, G., et al. (2014) Functional optimization of gene clusters by combinatorial design and assembly. Nat. Biotechnol., 32, 1241-1249.

Smith, M.D., Rounds, C.M., Wang, F., Chen, K., Afitlhile, M., and Schnell, D.J. (2004) atToc159 is a selective transit peptide receptor for the import of nucleus-encoded chloroplast proteins. J. Cell Biol., 165, 323-334.

Sood, P., Bhattacharya, A., and Sood, A. (2011) Problems and possibilities of monocot transformation. Biol. Plant., 55, 1-15.

Steinwand, M.A. and Ronald, P.C. (2020) Crop biotechnology and the future of food. Nat. Food, 1, 273-283.

Stewart, C.N., Patron, N., Hanson, A.D., and Jez, J.M. (2018) Plant metabolic engineering in the synthetic biology era: plant chassis selection. Plant Cell Rep., 37, 1357-1358.

Stewart, W.M. and Roberts, T.L. (2012) Food Security and the Role of Fertilizer in Supporting it. Procedia Eng., 46, 76-82.

Van Der Straeten, D., Bhullar, N.K., De Steur, H., Gruissem, W., MacKenzie, D., Pfeiffer, W., et al. (2020) Multiplying the efficiency and impact of biofortification through metabolic engineering. Nat. Commun., 11, 5203.

Sudhakar, D., Fu, X., Stoger, E., Williams, S., Spence, J., Brown, D.P., et al. (1998) Expression and immunolocalisation of the snowdrop lectin, GNA in transgenic rice plants. Transgenic Res., 7, 371-378.

Tello-Ruiz, M.K., Stein, J., Wei, S., Preece, J., Olson, A., Naithani, S., et al. (2016) Gramene 2016: Comparative plant genomics and pathway resources. Nucleic Acids Res., 44, D1133-D1140.

Temme, K., Zhao, D., and Voigt, C.A. (2012) Refactoring the nitrogen fixation gene cluster from Klebsiella oxytoca. Proc. Natl. Acad. Sci. U. S. A., 109, 7085-7090.

Towbin, H., Staehelin, T., and Gordon, J. (1979) Electrophoretic transfer of proteins from polyacrylamide gels to nitrocellulose sheets: procedure and some applications. Proc. Natl. Acad. Sci. U. S. A., 76, 4350-4.

Tsujimoto, R., Kotani, H., Yokomizo, K., Yamakawa, H., Nonaka, A., and Fujita, Y. (2018) Functional expression of an oxygen-labile nitrogenase in an oxygenic photosynthetic organism. Sci. Rep., 8, 7380.

Tzfira, T. and Citovsky, V. (2006) Agrobacterium-mediated genetic transformation of 
plants: biology and biotechnology. Curr. Opin. Biotechnol., 17, 147-154.

Ullrich, K.K., Hiss, M., and Rensing, S.A. (2015) Means to optimize protein expression in transgenic plants. Curr. Opin. Biotechnol., 32, 61-67.

Valdez, M. (1998) Transgenic Central American, West African and Asian Elite Rice Varieties Resulting from Particle Bombardment of Foreign DNA into Mature Seedderived Explants Utilizing Three Different Bombardment Devices. Ann. Bot., 82, 795-801.

Vaucheret, H. and Fagard, M. (2001) Transcriptional gene silencing in plants: targets, inducers and regulators. Trends Genet., 17, 29-35.

Vazquez-Vilar, M., Bernabé-Orts, J.M., Fernandez-del-Carmen, A., Ziarsolo, P., Blanca, J., Granell, A., and Orzaez, D. (2016) A modular toolbox for gRNA-Cas 9 genome engineering in plants based on the GoldenBraid standard. Plant Methods, 12, 10.

Vazquez-Vilar, M., Quijano-Rubio, A., Fernandez-del-Carmen, A., Sarrion-Perdigones, A., Ochoa-Fernandez, R., Ziarsolo, P., et al. (2017) GB3.0: a platform for plant biodesign that connects functional DNA elements with associated biological data. Nucleic Acids Res., 45, gkw1326.

Verma, M.L. and Rani, V. (2020) Biosensors for toxic metals, polychlorinated biphenyls, biological oxygen demand, endocrine disruptors, hormones, dioxin, phenolic and organophosphorus compounds: a review. Environ. Chem. Lett., 1-10.

Vicente, E.J. and Dean, D.R. (2017) Keeping the nitrogen-fixation dream alive. Proc. Natl. Acad. Sci., 114, 3009-3011.

Vojta, A., Alavi, M., Becker, T., Hörmann, F., Küchler, M., Soll, J., et al. (2004) The protein translocon of the plastid envelopes. J. Biol. Chem., 279, 21401-21405.

Waese, J., Fan, J., Pasha, A., Yu, H., Fucile, G., Shi, R., et al. (2017) ePlant: Visualizing and exploring multiple levels of data for hypothesis generation in plant biology. Plant Cell, 29, 1806-1821.

Wan, J., Blakeley, S.D., Dennis, D.T., and Ko, K. (1995) Import characteristics of a leucoplast pyruvate kinase are influenced by a 19-amino-acid domain within the protein. J. Biol. Chem., 270, 16731-16739.

Wan, J., Blakeley, S.D., Dennis, D.T., and Ko, K. (1996) Transit peptides play a major role in the preferential import of proteins into leucoplasts and chloroplasts. J. Biol. Chem., 271, 31227-31233.

Wang, L., Zhang, L., Liu, Z., Zhao, D., Liu, X., Zhang, B., et al. (2013) A Minimal Nitrogen Fixation Gene Cluster from Paenibacillus sp. WLY78 Enables Expression of Active Nitrogenase in Escherichia coli. PLoS Genet., 9, e1003865.

Ward, B.J., Gobeil, P., Séguin, A., Atkins, J., Boulay, I., Charbonneau, P.-Y., et al. (2020) Phase 1 trial of a Candidate Recombinant Virus-Like Particle Vaccine for Covid-19 Disease Produced in Plants. medRxiv, 2020.11.04.20226282.

Waters, M. and Pyke, K. (2005) Plastid Development and Differentiation. In: Annual Plant Reviews, pp. 30-59. Chichester, UK: John Wiley \& Sons, Ltd.

Weber, E., Engler, C., Gruetzner, R., Werner, S., and Marillonnet, S. (2011) A Modular Cloning System for Standardized Assembly of Multigene Constructs. PLoS One, 6, e16765.

Webster, G.R., Teh, A.Y.H., and Ma, J.K.C. (2017) Synthetic gene design-The rationale for codon optimization and implications for molecular pharming in plants. Biotechnol. Bioeng., 114, 492-502.

Werner, S., Engler, C., Weber, E., Gruetzner, R., and Marillonnet, S. (2012) Fast track assembly of multigene constructs using Golden Gate cloning and the MoClo system. Bioengineered, 3, 38-43.

Wilson, S.A. and Roberts, S.C. (2014) Metabolic engineering approaches for production 
of biochemicals in food and medicinal plants. Curr. Opin. Biotechnol., 26, 174-182. Wise, R.R. (2007) The Diversity of Plastid Form and Function. In: The Structure and Function of Plastids, pp. 3-26.

Witte, C.-P. (2011) Urea metabolism in plants. Plant Sci., 180, 431-438.

Xiang, N., Guo, C., Liu, J., Xu, H., Dixon, R., Yang, J., and Wang, Y.-P. (2020) Using synthetic biology to overcome barriers to stable expression of nitrogenase in eukaryotic organelles. Proc. Natl. Acad. Sci. U. S. A., 117, 16537-16545.

Yan, A., Wang, Y., Tan, S.N., Mohd Yusof, M.L., Ghosh, S., and Chen, Z. (2020) Phytoremediation: A Promising Approach for Revegetation of Heavy MetalPolluted Land. Front. Plant Sci., 11, 359.

Yan, X., Khan, S., Hase, T., Emes, M.J., and Bowsher, C.G. (2006) Differential uptake of photosynthetic and non-photosynthetic proteins by pea root plastids. FEBS Lett., 580, 6509-6512.

Yang, J., Xie, X., Yang, M., Dixon, R., and Wang, Y.-P. (2017) Modular electrontransport chains from eukaryotic organelles function to support nitrogenase activity. Proc. Natl. Acad. Sci., 114, E2460-E2465.

Yonekura-Sakakibara, K., Ashikari, T., Tanaka, Y., Kusumi, T.A., and Hase, T. (1998) Molecular characterization of tobacco sulfite reductase: Enzyme purification, gene cloning, and gene expression analysis. J. Biochem., 124, 615-621.

Yoo, S.-D., Cho, Y.-H., and Sheen, J. (2007) Arabidopsis mesophyll protoplasts: a versatile cell system for transient gene expression analysis. Nat. Protoc., 2, 15651572.

Yoon, H.S., Hackett, J.D., Ciniglia, C., Pinto, G., and Bhattacharya, D. (2004) A Molecular Timeline for the Origin of Photosynthetic Eukaryotes. Mol. Biol. Evol., 21, 809-818.

Yu, K., Ang, K.S., and Lee, D.-Y. (2017) Synthetic Gene Design Using Codon Optimization On-Line (COOL). In: Methods in Molecular Biology, pp. 13-34. Humana Press Inc.

Yuvaniyama, P., Agar, J.N., Cash, V.L., Johnson, M.K., and Dean, D.R. (2000) NifSdirected assembly of a transient [2Fe-2S] cluster within the NifU protein. Proc. Natl. Acad. Sci. U. S. A., 97, 599-604.

Zehr, J.P. (2013) Interactions with Partners Are Key for Oceanic Nitrogen-Fixing Cyanobacteria. Microbe Mag., 8, 117-122.

Zhang, Y., Su, J., Duan, S., Ao, Y., Dai, J., Liu, J., et al. (2011) A highly efficient rice green tissue protoplast system for transient gene expression and studying light/chloroplast-related processes. Plant Methods, 7, 30.

Zheng, C. and Dos Santos, P.C. (2018) Metallocluster transactions: Dynamic protein interactions guide the biosynthesis of $\mathrm{Fe}-\mathrm{S}$ clusters in bacteria. Biochem. Soc. Trans., 46, 1593-1603.

Zheng, L., White, R.H., Cash, V.L., and Dean, D.R. (1994) Mechanism for the desulfurization of L-cysteine catalyzed by the nifS gene product. Biochemistry, 33, 4714-20.

Zheng, L., White, R.H., Cash, V.L., Jack, R.F., and Dean, D.R. (1993) Cysteine desulfurase activity indicates a role for NIFS in metallocluster biosynthesis. Proc. Natl. Acad. Sci., 90, 2754-2758.

Zhong, H., Teymouri, F., Chapman, B., Maqbool, S.B., Sabzikar, R., El-Maghraby, Y., et al. (2003) The pea (Pisum sativum L.) rbcS transit peptide directs the Alcaligenes eutrophus polyhydroxybutyrate enzymes into the maize (Zea mays L.) chloroplasts. Plant Sci., 165, 455-462.

Zhu, C., Naqvi, S., Breitenbach, J., Sandmann, G., Christou, P., and Capell, T. (2008) 
Combinatorial genetic transformation generates a library of metabolic phenotypes for the carotenoid pathway in maize. Proc. Natl. Acad. Sci., 105, 18232-18237.

Zybailov, B., Rutschow, H., Friso, G., Rudella, A., Emanuelsson, O., Sun, Q., and van Wijk, K.J. (2008) Sorting signals, N-terminal modifications and abundance of the chloroplast proteome. PLoS One, 3, e1994. 


\section{Supplemental Material}

Supplemental Table 1. List of primers used in this thesis.

\begin{tabular}{|c|c|c|}
\hline $\begin{array}{l}\text { Primer } \\
\text { number }\end{array}$ & Primer sequence $\left(5^{\prime}-3^{\prime}\right)$ & Comment \\
\hline 406 & ATGAAGACATCTCGGGCTGCTCGTGGATGCTGCTGCGTT & \\
\hline 407 & ATGAAGACATCTCAAATGGCGCAAGTTAGCAGAATC & \\
\hline 408 & GCGCGAAGACTGAAAACGCAGCAGCATCCACG & Mutation G $105>$ A \\
\hline 409 & ATGAAGACATCTCGGGCTTCCGCCGTGGAAACAGACGACATGACC & Mutation T $210>\mathrm{G}$ \\
\hline 410 & GCGCGAAGACCGTTTTCAGAGAAACCGATAAGGG & Mutation G $105>$ A \\
\hline 411 & ATGAAGACATCTCGGGCTGCTTTCACCACAGGGTAGC & \\
\hline 412 & ATGAAGACATCTCAAATGGCGTCGTCGTCGTTCTC & Mutation $\mathrm{T} 9>\mathrm{G}$ \\
\hline 413 & GCGCGAAGACGTCTTTCTGCTAAGCCCAAGCT & Mutation $\mathrm{C} 120>\mathrm{T}$ \\
\hline 414 & ATGAAGACATCTCGGGCTCCATCAACTTTGGCAGCAT & \\
\hline 415 & GCGCGAAGACAGAAAGACGGAAAGAAACTCTGC & Mutation C $120>\mathrm{T}$ \\
\hline 416 & ATGAAGACATCTCGGGCTGCTGGTCGTGGCTCGCCAG & \\
\hline 417 & ATGAAGACATCTCAAATGGCGTCGAACTCGCTTATG & \\
\hline 418 & ATGAAGACATCTCGGGCTCCAAAGTCACCAGGAGCAG & \\
\hline 419 & ATGAAGACATCTCGGGCTGAACAAACGACTTTTGATCTGT & \\
\hline 420 & ATGAAGACATCTCAAATGACAATTGCTTTAACGATCGG & \\
\hline 421 & ATGAAGACATCTCGGGCTTTAGCGAGTTGTCTGAAAGCT & \\
\hline 422 & ATGAAGACATCTCGGGCTGCTTTCACCGAAAACGGCGAC & \\
\hline 423 & ATGAAGACATCTCAAATGGCTCTACAGTCTCCCGGAG & \\
\hline 424 & ATGAAGACATCTCGGGCTGCTCGGTCAGAATTAAGGATCG & \\
\hline 425 & ATTAAGGATCGCTTTCACCGAAAACGGCGACCTCAGCGAG & Mutation T $201>\mathrm{G}$ \\
\hline 426 & ATGAAGACATCTCGGGCTGCAGCACTAGCACGAGTAG & \\
\hline 427 & ATGAAGACATCTCAAATGGCTTCTTCTATGCTTTCTT & \\
\hline 428 & ATGAAGACATCTCGGGCTGCTCGAACTCTTCCTCCGTTA & \\
\hline 432 & ATGAAGACATCTCGGGCTGCTTGAGCAACATTGCTGC & \\
\hline 433 & ATGAAGACATCTCAAATGGCTTCCTCAGTTCTTTCCT & \\
\hline
\end{tabular}




\begin{tabular}{|c|c|c|}
\hline 434 & ATGAAGACATCTCGGGCTGAGCATTGCACTCTTCCGCCGT & \\
\hline 435 & ATGAAGACATCTCGGGCTGAGCAGTTAACTCTTCCGCCGT & \\
\hline 436 & ATGAAGACATCTCAAATGGCTTCCTCTATGCTCTCT & \\
\hline 437 & ATGAAGACATCTCGGGCTGAATCGGTAAGGTCAGGAAGG & \\
\hline 438 & ATGAAGACATCTCGGGCTGCGGATTGCCCCCTAGAGG & \\
\hline 439 & ATGAAGACATCTCAAATGGCTTCTATGATATCCTCTTCC & \\
\hline 440 & ATGAAGACATCTCGGGCTGAGCACTTTACTCTTCCACCAT & \\
\hline 441 & ATGAAGACATCTCGGGCTGCCAACACCTTGAATGATCC & \\
\hline 442 & ATGAAGACATCTCAAATGGCCGCCGCAGTTTCCAC & \\
\hline 443 & GCGCGAAGACAAGATAAACAAACCGATGGAGAC & Mutation C $189>\mathrm{T}$ \\
\hline 444 & ATGAAGACATCTCGGGCTGAATCAGAAGTGTCGTAGGCAA & \\
\hline 445 & GCGCGAAGACTTTATCTTCTTTCACAGCCAACAC & Mutation C $189>\mathrm{T}$ \\
\hline 446 & ATGAAGACATCTCGGGCTGCGCGGACAATAGAACTAGG & \\
\hline 447 & ATGAAGACATCTCAAATGACGACGTCGTTTGGAGC & \\
\hline 448 & GCGCGAAGACGTCTGCACGTTTTTCAGTCCTTT & Mutation $\mathrm{T} 126>\mathrm{G}$ \\
\hline 449 & ATGAAGACATCTCGGGCTGACGTAGATACGGCGCGGACAA & \\
\hline 450 & GCGCGAAGACGTGCAGACGCCTGCTAAGCAAC & Mutation T $126>\mathrm{G}$ \\
\hline 451 & ATGAAGACATCTCGGGCTGCCGAAACCCTAGAAATGG & \\
\hline 452 & ATGAAGACATCTCAAATGGAGATACGGAGCTTGATTG & \\
\hline 453 & ATGAAGACATCTCGGGCTGAGTGAGGAGTCCGGAGTT & \\
\hline 454 & ATGAAGACATCTCATTCGATGGCCATGAGACAATGTGC & \\
\hline 455 & ATGAAGACATCTCGAAGCTTATACTTCTTCAGCAGTTTTACCG & \\
\hline 456 & ATGAAGACATCTCAAGCCATGGCCTCAGAAAGATTAG & \\
\hline 457 & ATGAAGACATCTCGAAGCTTAACCATGTGCTAAGTTTTC & \\
\hline 458 & ATGAAGACATCTCAAGCCATGGCCGACGTTTACTTGGA & \\
\hline 459 & ATGAAGACATCTCGAAGCTCAACCATAGACAGGAGCAAAG & \\
\hline 460 & AGACAGGAGCAAAGGCTTTACCAGGATCTTCAACAG & Mutation C $1176>\mathrm{T}$ \\
\hline 461 & ATGAAGACATCTCAAGCCATGTGGGACTACTCTGAAAAGG & \\
\hline 462 & ATGAAGACATCTCGAAGCTCAGACTTCCATTTGGGCGTG & \\
\hline 463 & ATGAAGACATCTCATTCGATGGCAATGAGACAATGTGCTA & \\
\hline
\end{tabular}




\begin{tabular}{|c|c|c|}
\hline 465 & ATGAAGACATCTCAAGCCATGGCATCAGAAAGATTAGCTG & \\
\hline 467 & ATGAAGACATCTCAAGCCATGGCAGACGTTTATTTGGACA & \\
\hline 469 & ATGAAGACATCTCAAGCCATGTGGGATTACTCAGAGAAAG & \\
\hline 471 & ATGAAGACATCTCAAGCCTCTGCATGGAGTCATCCTCAG & \\
\hline 479 & ATGAAGACATCTCAGGAGGAATTCCAATCCCACA & \\
\hline 480 & ATGAAGACATCTCGCATTGCGTGTCCTCTCCAAATGA & \\
\hline 495 & ATGAAGACATCTCGCGAAGATTTTTCAAATTGTGGATGTG & \\
\hline 502 & ATGAAGACATCTCGAAGCTTACACTTCCTCAGCGGTTT & \\
\hline 503 & ATGAAGACATCTCGAAGCTTATCCATGCGCCAAATTCTCT & \\
\hline 504 & ATGAAGACATCTCGAAGCTTATCCGTAAACGGGCGCGAA & \\
\hline 505 & ATGAAGACATCTCGAAGCTTATACTTCCATCTGAGCATG & \\
\hline 535 & ATGAAGACATCTCGGGCTGCGTGTCCTCTCCAAATGA & \\
\hline 576 & ATGAAGACATCTCAGCAGATGAGAGGATCTGGATCTG & \\
\hline 577 & ATGAAGACATCTCGAAGCCTATTCCTCACCAGCATCAGC & \\
\hline 578 & ATGAAGACATCTCGCTGCCCACCGCTTCCACCACCT & Partial linker \\
\hline 579 & CTTCCACCACCTGAACCTCCCACTTCCTCAGCGGTTTTGC & $\begin{array}{l}\text { No stop codon. Partial } \\
\text { linker }\end{array}$ \\
\hline 580 & СТTCCACCACCTGAACCTCCTCCATGCGCCAAATTCTCTA & $\begin{array}{l}\text { No stop codon. Partial } \\
\text { linker }\end{array}$ \\
\hline 581 & СТTCCACCACCTGAACCTCCTCCGTAAACGGGCGCG & $\begin{array}{l}\text { No stop codon. Partial } \\
\text { linker }\end{array}$ \\
\hline 582 & CTTCCACCACCTGAACCTCCTACTTCCATCTGAGCATGTG & $\begin{array}{l}\text { No stop codon. Partial } \\
\text { linker }\end{array}$ \\
\hline 595 & ATGAAGACATCTCAGGAGTGCAGTGCAGCGTGACCCGGTC & \\
\hline 596 & ATGAAGACATCTCGCATTCTGCAGAAGTAACACCAAACAA & \\
\hline 597 & ATGAAGACATCTCAGGAGTAGCTAGCATACTCGAGGTCAT & \\
\hline 598 & GCGCCGTCTCGCAAGACCGAGACGAAAAAAAAAAC & \\
\hline 599 & GCGCCGTCTCGCTTGATCTTTGGCCTTGGTAG & \\
\hline 600 & ATGAAGACATCTCGCATTCTTCTACCTACAAAAAAGCTCC & \\
\hline 613 & ATGAAGACATCTCAAGCCTGGGACTACAGCGAGAAGGTGA & \\
\hline 614 & ATGAAGACATCTCGAAGCTCAGACCTCCATCTGGGCATGG & \\
\hline 615 & ATGAAGACATCTCAAGCCGCGGACGTGTACCTCGATAACA & \\
\hline 616 & ATGAAGACATCTCGAAGCTCATCCGTAGACAGGGGCGAAT & \\
\hline 617 & ATGAAGACATCTCAAGCCGCCTCAGAGAGGCTCGCGGACG & \\
\hline
\end{tabular}




\begin{tabular}{|c|c|c|}
\hline 618 & ATGAAGACATCTCGAAGCTCAGCCGTGCGCCAGATTCTCG & \\
\hline 621 & ATGAAGACATCTCAAGCCGTGAGCAAGGGCGAGGAGCTGT & \\
\hline 622 & ATGAAGACATCTCGAAGCTTACTTGTACAGCTCGTCCATG & \\
\hline 623 & ATGAAGACATCTCATTCGGCCATGAGGCAGTGCGCGATCT & \\
\hline 624 & ATGAAGACATCTCGAAGCTCACACTTCCTCGGCCGTCTTG & \\
\hline 629 & ATGAAGACATCTCAGCTTATGGGGCATCGGCGACGAGCAC & \\
\hline 630 & ATGAAGACATCTCGAGCGTCGATCTGCCCAGTTAAGATCA & \\
\hline 712 & ATGAAGACATCTCAGCAGATGAGCGAGCTGATTAAGGAGA & \\
\hline 713 & ATGAAGACATCTCGAAGCTCAATTAAGCTTGTGCCCCAG & \\
\hline 714 & ATGAAGACATCTCAGCAGATGGTGAGCAAGGGC & \\
\hline 715 & ATGAAGACATCTCGAAGCTCACTTGTACAGCTCGTCCA & \\
\hline 758 & ATGAAGACATCTCGGGCTGACATTCTGCAGAAGTAACACC & \\
\hline 759 & ATGAAGACATCTCAAATGGCCCCCTCCGTGATG & \\
\hline 760 & ATGAAGACATCTCGGGCTGCGCACCTGATCCTGCCGCCAT & \\
\hline 765 & ATGAAGACATCTCAAATGAAAAAGCCTGAACTCACC & \\
\hline 766 & ATGAAGACATCTCGAAGCTATTCCTTTGCCCTCGGAC & \\
\hline 771 & ATGAAGACATCTCAAGCCTCTGCCTGGTCACACCCT & \\
\hline 772 & ATGAAGACATCTCGCGAAGATTTCTCGAACTGCGGATGG & \\
\hline 812 & ATGAAGACATCTCAGGAGGTCAACATGGTGGAGC & \\
\hline 813 & ATGAAGACATCTCATTCGATGGCTATGCGTCAATGCG & \\
\hline 814 & $\begin{array}{l}\text { ATGAAGACATCTCGAAGCTCAGACTTCTTCGGCGGTTTTGCCGAC- } \\
\text { GATGGATTCATCTTCG }\end{array}$ & \\
\hline 815 & ATGAAGACATCTCAAGCCATGGCATCTGAGCGTCTCG & \\
\hline 816 & ATGAAGACATCTCGAAGCTCATCCATGGGCGAGGTTC & \\
\hline 817 & ATGAAGACATCTCAAGCCATGTGGGATTATTCGGAAAAAG & \\
\hline 818 & ATGAAGACATCTCGAAGCTCAGACCTCCATCTGCGC & \\
\hline 819 & ATGAAGACATCTCAAGCCATGGCTGACGTCTATCTCG & \\
\hline 820 & ATGAAGACATCTCGAAGCTCAGCCGTAGACCG & \\
\hline 823 & GCGCGAAGACTTCATCTTCCAGCTCCAGATCT & Mutation C $225>\mathrm{T}$ \\
\hline 824 & GCGCGAAGACAAGATGTGCTGAAGGCTGGCTA & Mutation C $225>\mathrm{T}$ \\
\hline 825 & GCGCGAAGACTTTCATCTTCGTAGGCGCCTTC & Mutation C $354>\mathrm{T}$ \\
\hline
\end{tabular}




\begin{tabular}{|c|c|c|}
\hline 826 & GCGCGAAGACAAATGATCTGGACTTCGTATTCTAC & Mutation C $354>\mathrm{T}$ \\
\hline 827 & GCGCGAAGACTTCTCATCTTCGCGGTCGGTGT & Mutation C $582>\mathrm{T}$ \\
\hline 828 & GCGCGAAGACAATGAGCTGATCATCGCTCTGG & Mutation C $582>\mathrm{T}$ \\
\hline 829 & GCGCGAAGACTTTTGTCTCGATGCGCGTCCGG & Mutation C $516>$ A \\
\hline 830 & GCGCGAAGACAAACAATCCTCAAGCGGCTGCG & Mutation C $516>$ A \\
\hline 831 & GCGCGAAGACTTCAAAGACTTCACCGCGAGCG & Mutation C $204>\mathrm{T}$ \\
\hline 832 & GCGCGAAGACAATTTGTCGCGGCCCCGATAAA & Mutation C $204>$ T \\
\hline 833 & GCGCGAAGACTTCTGTCTCGATGCGACGGATG & Mutation C $714>$ A \\
\hline 834 & GCGCGAAGACAAACAGTGCTGGCGGCGATCCG & Mutation C $714>$ A \\
\hline 835 & GCGCGAAGACTTTAAAGACGATTTCCGAATCGTG & Mutation C $204>$ T \\
\hline 836 & GCGCGAAGACAATTTACCTCCTGCGGCACCGA & Mutation C $204>\mathrm{T}$ \\
\hline 837 & GCGCGAAGACTTCTGTCTCGTTGTTGGCCCAC & Mutation C $459>$ A \\
\hline 838 & GCGCGAAGACAAACAGGTACCCTGTTCCCGAT & Mutation C $459>$ A \\
\hline 883 & СТTCCACCACCTGAACCTCCCACTTCCTCGGCCGTCTTG & $\begin{array}{l}\text { No stop codon. Partial } \\
\text { linker }\end{array}$ \\
\hline 884 & CTTCCACCACCTGAACCTCCGCCGTGCGCCAGATTCTC & $\begin{array}{c}\text { No stop codon. Partial } \\
\text { linker }\end{array}$ \\
\hline 887 & GCACTTCACGCCACCGTATC & $\begin{array}{c}\text { Overlapping PCR } \\
\text { Mutation C 243 > T }\end{array}$ \\
\hline 888 & GATACGGTGGCGTGAAGTGC & $\begin{array}{c}\text { Overlapping PCR } \\
\text { Mutation C } 243>\mathrm{T}\end{array}$ \\
\hline 889 & CTGTGATGACTCCGCGGC & $\begin{array}{c}\text { Overlapping PCR } \\
\text { Mutation C 303 > A }\end{array}$ \\
\hline 890 & GCCGCGGAGTCATCACAG & $\begin{array}{c}\text { Overlapping PCR } \\
\text { Mutation C 303 > A }\end{array}$ \\
\hline 891 & GAATCCGCCACAGACCACG & $\begin{array}{c}\text { Overlapping PCR } \\
\text { Mutation C 396 > A }\end{array}$ \\
\hline 892 & CGTGGTCTGTGGCGGATTC & $\begin{array}{c}\text { Overlapping PCR } \\
\text { Mutation C 396 > A }\end{array}$ \\
\hline 893 & CACTCACTGTGCTTCATGGC & $\begin{array}{c}\text { Overlapping PCR } \\
\text { Mutation TTCA } 570> \\
\text { CAGT }\end{array}$ \\
\hline 894 & CTCAGCTCACCGCGGGCCATC & $\begin{array}{c}\text { Overlapping PCR } \\
\text { Mutation TTCA } 570> \\
\text { CAGT }\end{array}$ \\
\hline 895 & GCCATGAAGCACAGTGAGTG & $\begin{array}{c}\text { Overlapping PCR } \\
\text { Mutation C } 672>\mathrm{T}\end{array}$ \\
\hline 896 & GATGGCCCGCGGTGAGCTGAG & $\begin{array}{c}\text { Overlapping PCR } \\
\text { Mutation C 243 > T }\end{array}$ \\
\hline 897 & ACCCGATCTATTTCTTCCTCCG & $\begin{array}{c}\text { Overlapping PCR } \\
\text { Mutation G } 1083>\mathrm{A} \\
\& \mathrm{C} 1086>\mathrm{A}\end{array}$ \\
\hline 898 & CGGAGGAAGAAATAGATCGGGT & $\begin{array}{c}\text { Overlapping PCR } \\
\text { Mutation G } 1083>\mathrm{A} \\
\& \mathrm{C} 1086>\mathrm{A}\end{array}$ \\
\hline
\end{tabular}




\begin{tabular}{|c|c|c|}
\hline 899 & TCCCCACAGGACAGTGAGC & $\begin{array}{c}\text { Overlapping PCR } \\
\text { Mutation C } 102>\text { T \& } \\
\text { C } 105>\mathrm{G}\end{array}$ \\
\hline 900 & GCTCACTGTCCTGTGGGGA & $\begin{array}{c}\text { Overlapping PCR } \\
\text { Mutation C } 102>\mathrm{T} \& \\
\text { C } 105>\mathrm{G}\end{array}$ \\
\hline 901 & GTCCTCGATTGTCTCTCCGC & $\begin{array}{c}\text { Overlapping PCR } \\
\text { Mutation C } 366>\text { A }\end{array}$ \\
\hline 902 & GCGGAGAGACAATCGAGGAC & $\begin{array}{c}\text { Overlapping PCR } \\
\text { Mutation C } 366>\text { A }\end{array}$ \\
\hline 903 & CATGCAGAGCAGCCACCG & $\begin{array}{c}\text { Overlapping PCR } \\
\text { Mutation C 507 > T }\end{array}$ \\
\hline 904 & CGGTGGCTGCTCTGCATG & $\begin{array}{c}\text { Overlapping PCR } \\
\text { Mutation C 507 > T }\end{array}$ \\
\hline 905 & GAGCACTGTCTCAATACTGCG & $\begin{array}{c}\text { Overlapping PCR } \\
\text { Mutation G } 702>\text { T \& } \\
\text { C } 705>\text { T }\end{array}$ \\
\hline 906 & CGCAGTATTGAGACAGTGCTC & $\begin{array}{c}\text { Overlapping PCR } \\
\text { Mutation G } 702>\text { T \& } \\
\text { C } 705>\text { T }\end{array}$ \\
\hline 907 & TCGACGTCGATTAGCTCCAC & $\begin{array}{c}\text { Overlapping PCR } \\
\text { Mutation G } 765>\mathrm{A}\end{array}$ \\
\hline 908 & GTGGAGCTAATCGACGTCG & $\begin{array}{c}\text { Overlapping PCR } \\
\text { Mutation G } 765>\mathrm{A}\end{array}$ \\
\hline 909 & CTCCTCAATGAGCCGCTGC & $\begin{array}{c}\text { Overlapping PCR } \\
\text { Mutation G } 864>\mathrm{C} \& \\
\text { C } 867>\mathrm{T}\end{array}$ \\
\hline 910 & GCAGCGGCTCATTGAGGA & $\begin{array}{c}\text { Overlapping PCR } \\
\text { Mutation G } 864>\text { C \& } \\
\text { C } 867>\text { T }\end{array}$ \\
\hline 947 & ATGAAGACATCTCAAATGGATTCACAGCTAGTCTTG & \\
\hline 948 & ATGAAGACATCTCGGGCTGCGGCGTAGACGGTAACCGGAG & \\
\hline
\end{tabular}


Supplemental Table 1 (continued). List of primers used in this thesis.

\begin{tabular}{|c|c|c|}
\hline $\begin{array}{l}\text { Primer } \\
\text { number }\end{array}$ & $\begin{array}{l}\text { Primer sequence }\left(\mathbf{5}^{\prime}-\mathbf{3}^{\prime}\right) \\
\text { Internal primers used for Sanger sequencing. }\end{array}$ & Comment \\
\hline 261 & AGCGAGGAAGCGGAAGAGCG & $\begin{array}{l}\text { L1Px_pUC57 } \\
\text { Fwd } \\
\end{array}$ \\
\hline 262 & GCCACCTGACGTCTAAGAAACC & L1Px_pUC57 \\
\hline 263 & GGATAAACCTTTTCACGCCC & MoClo RB \\
\hline 266 & GTGGTGTAAACAAATTGACGC & MoClo LB \\
\hline 393 & ACTTCAAGATCCGCCACAACA & GFP Fwd \\
\hline 394 & ATGCCGTTCTTCTGCTTGTC & GFP Rev \\
\hline 405 & GACACCGCGCGCGATAATTT & tNOS Rev \\
\hline 475 & GGACAGGTATCCGGTAAGCG & $\begin{array}{l}\text { pAGM9121 (L0) } \\
\text { Fwd }\end{array}$ \\
\hline 476 & AACAAGCCATGAAAACCGCC & $\begin{array}{c}\text { pAGM9121 (L0) } \\
\text { Rev }\end{array}$ \\
\hline 526 & TCATACCGTGACTCCCTTAATTCTC & pNOS Rev \\
\hline 527 & AGCGCGCAAACTAGGATAAATTATC & tNOS Fwd \\
\hline 528 & TCCTAAAACCAAAATCCAGTACT & t35S Fwd \\
\hline 583 & AACAGGCTGATGAGTACCGC & nifH tsd Fwd \\
\hline 584 & CCTTCGGATCACAGCCAACT & nifH tsd Rev \\
\hline 585 & GGCTAGGGGCGAATTATCCC & nif $M$ tsd Fwd \\
\hline 586 & CTAACGCCTGCTGAAGAGCT & nifM tsd Rev \\
\hline 587 & ACATGCCTTCGTCACAGGAG & nifS tsd Fwd \\
\hline 588 & AAGATTGCGGTGCTGTCAGA & nifS tsd Rev \\
\hline 589 & CGCAAGGGGTGAAGTGTTTG & nifU tsd Fwd \\
\hline 590 & GCTTCGTCCAATGTCAAGCC & nif $U$ tsd Rev \\
\hline 698 & GCTAGCGTTCGTACACGGAT & $\begin{array}{l}\text { pMaize Ubi1+1i } \\
\text { Fwd }\end{array}$ \\
\hline 739 & CCGTCAGGGCGCGTC & L2_pUC57 Fwd \\
\hline 746 & CACGCGACAATGTGGTCC & nifH rco Fwd \\
\hline 747 & TCGGCCGCCATCTCCA & nifH rco Rev \\
\hline 748 & CGAGTCTCCTATCGGCTTCC & nifM rco Fwd \\
\hline 749 & CCTCTGGTGACTCGTAGCG & nifM rco Rev \\
\hline 750 & СCTTCACACGTGATGAGGGC & nifS rco Fwd \\
\hline
\end{tabular}




\begin{tabular}{c|l|c}
\hline 751 & GCTGCGCCTTCAGGG & nifS rco Rev \\
\hline 752 & CCTCCAGCGGGATAAGGG & nifU roo Fwd \\
\hline 753 & GAAGGTCTGGAAGCCGGC & nifU rco Rev \\
\hline 870 & ACTGTCGGGCGTACACAAAT & HPT Fwd \\
\hline 905 & CGATGTCGACGGCAAGAACGTTTATGTCAA & nifS nco Fwd \\
\hline 908 & CATCCTGCTGCTGCTGAACAAGGTC & nifU nco Fwd \\
\hline 911 & CATACAAAAATGACGTATCAAAG & pRice Actin1 \\
\hline 928 & TGAGTCCGGTACTGGAATCG & nifM nco Fwd \\
\hline 936 & AGAGCATTGGCCAGAAAGGT & nifH yco Fwd \\
\hline 936 & CCGCCAACAACATCTCCAA & nifH nco Fwd \\
\hline 937 & CCAGCAAGACAATTGACCTTGG & nifM yco Fwd \\
\hline 940 & TACAGCTGCACACGGTACTG & nifS yco Fwd \\
\hline 941 & ACGTCAAATTGACCGGTGCT & nifU yco Fwd \\
\hline 942 & CGCGAACTCTTCAGCATCTC & P19 Fwd \\
\hline 1429 & GACGTAAGGGATGACGCACA & p35S Fwd \\
\hline 2288 & CTACGAGGGCACCCAGACC & BFP Fwd \\
\hline 2289 & CTGCCACCTCGTGCTGCT & BFP Rev \\
\hline
\end{tabular}


Supplemental Table 2. List of modular pieces generated in this thesis.

\begin{tabular}{|c|c|c|c|c|c|c|}
\hline Modular piece & Backbone & Position & Type & $\begin{array}{l}\text { Domesticated } \\
\text { for }\end{array}$ & $\begin{array}{l}\text { Amplification } \\
\text { Primers } \\
\text { (Mutagenesis } \\
\text { primers) } \\
\end{array}$ & Template \\
\hline AtmAROA & pAGM9121 & B3 & $\mathrm{TP}$ & BsaI, BpiI & $\begin{array}{c}406 / 407 \\
(408 / 410)\end{array}$ & $\begin{array}{l}\text { Arabidopsis } \\
\text { genomic DNA }\end{array}$ \\
\hline $\operatorname{AtcAROA}_{\mathrm{TP}}$ & pAGM9121 & B3 & $\mathrm{TP}$ & BsaI, BpiI & $\begin{array}{c}406 / 409 \\
(409)\end{array}$ & $\begin{array}{l}\text { Arabidopsis } \\
\text { genomic DNA }\end{array}$ \\
\hline$A t \mathrm{mBCCP} 1_{\mathrm{TP}}$ & pAGM9121 & B3 & $\mathrm{TP}$ & BsaI, BpiI & $\begin{array}{c}411 / 412 \\
(413 / 415 / 412)\end{array}$ & $\begin{array}{c}\text { Arabidopsis } \\
\text { cDNA }\end{array}$ \\
\hline$A t \mathrm{cBCCP} 1_{\mathrm{TP}}$ & pAGM9121 & B3 & $\mathrm{TP}$ & BsaI, BpiI & $\begin{array}{c}414 / 412 \\
(413 / 415 / 412)\end{array}$ & $\begin{array}{c}\text { Arabidopsis } \\
\text { cDNA }\end{array}$ \\
\hline AtmDNAJ8 $8_{\mathrm{TP}}$ & pAGM9121 & B3 & $\mathrm{TP}$ & BsaI, BpiI & $419 / 420$ & $\begin{array}{l}\text { Arabidopsis } \\
\text { genomic DNA }\end{array}$ \\
\hline AtcDNAJ8 ${ }_{\mathrm{TP}}$ & pAGM9121 & B3 & $\mathrm{TP}$ & BsaI, BpiI & $421 / 420$ & $\begin{array}{l}\text { Arabidopsis } \\
\text { genomic DNA }\end{array}$ \\
\hline AtmCAB6 ${ }_{\mathrm{TP}}$ & pAGM9121 & B3 & $\mathrm{TP}$ & BsaI, BpiI & $416 / 417$ & $\begin{array}{l}\text { Arabidopsis } \\
\text { genomic DNA }\end{array}$ \\
\hline$A t c \mathrm{CAB} 6_{\mathrm{TP}}$ & pAGM9121 & B3 & $\mathrm{TP}$ & BsaI, BpiI & $418 / 417$ & $\begin{array}{l}\text { Arabidopsis } \\
\text { genomic DNA }\end{array}$ \\
\hline AtmGLTB2 $2_{\mathrm{TP}}$ & pAGM9121 & B3 & $\mathrm{TP}$ & BsaI, BpiI & $\begin{array}{c}422 / 423 \\
(425)\end{array}$ & $\begin{array}{l}\text { Arabidopsis } \\
\text { genomic DNA }\end{array}$ \\
\hline$A t c G L T B 22_{\mathrm{TP}}$ & pAGM9121 & B3 & $\mathrm{TP}$ & BsaI, BpiI & $\begin{array}{c}423 / 424 \\
(425)\end{array}$ & $\begin{array}{l}\text { Arabidopsis } \\
\text { genomic DNA }\end{array}$ \\
\hline$N t \mathrm{mRBS}_{\mathrm{TP}}$ & pAGM9121 & B3 & $\mathrm{TP}$ & BsaI, BpiI & $432 / 433$ & $\begin{array}{c}\text { Nicotiana } \\
\text { tabacum } \\
\text { genomic DNA } \\
\text { Nicotiana } \\
\text { tabacum } \\
\text { genomic DNA }\end{array}$ \\
\hline AtmRBS1 $\mathrm{A}_{\mathrm{TP}}$ & pAGM9121 & B3 & $\mathrm{TP}$ & BsaI, BpiI & $435 / 436$ & $\begin{array}{c}\text { Arabidopsis } \\
\text { cDNA }\end{array}$ \\
\hline AtcRBS1 $\mathrm{A}_{\mathrm{TP}}$ & pAGM9121 & B3 & $\mathrm{TP}$ & BsaI, BpiI & $437 / 436$ & $\begin{array}{c}\text { Arabidopsis } \\
\text { cDNA }\end{array}$ \\
\hline$P s \mathrm{mRBS} 2_{\mathrm{TP}}$ & pAGM9121 & B3 & $\mathrm{TP}$ & BsaI, BpiI & $438 / 439$ & $\begin{array}{l}\text { Pisum sativum } \\
\text { genomic DNA }\end{array}$ \\
\hline$P s \mathrm{cRBS} 2_{\mathrm{TP}}$ & pAGM9121 & B3 & $\mathrm{TP}$ & BsaI, BpiI & $440 / 439$ & $\begin{array}{l}\text { Pisum sativum } \\
\text { genomic DNA }\end{array}$ \\
\hline AtmRCA & pAGM9121 & B3 & $\mathrm{TP}$ & BsaI, BpiI & $\begin{array}{c}441 / 442 \\
(443 / 445)\end{array}$ & $\begin{array}{c}\text { Arabidopsis } \\
\text { cDNA }\end{array}$ \\
\hline$A t c \mathrm{RCA}_{\mathrm{TP}}$ & pAGM9121 & B3 & $\mathrm{TP}$ & BsaI, BpiI & $\begin{array}{c}444 / 442 \\
(443 / 445)\end{array}$ & $\begin{array}{c}\text { Arabidopsis } \\
\text { cDNA }\end{array}$ \\
\hline$N t \mathrm{mSIR}_{\mathrm{TP}}$ & pAGM9121 & B3 & TP & BsaI, BpiI & $\begin{array}{c}446 / 447 \\
(448 / 450)\end{array}$ & $\begin{array}{c}\text { Nicotiana } \\
\text { tabacum } \\
\text { genomic DNA }\end{array}$ \\
\hline
\end{tabular}




\begin{tabular}{|c|c|c|c|c|c|c|}
\hline$N t \mathrm{cSIR} \mathrm{TP}_{\mathrm{T}}$ & pAGM9121 & B3 & $\mathrm{TP}$ & BsaI, BpiI & $\begin{array}{c}449 / 447 \\
(448 / 450)\end{array}$ & $\begin{array}{c}\text { Nicotiana } \\
\text { tabacum } \\
\text { genomic DNA }\end{array}$ \\
\hline$A t \mathrm{mTOCC} \mathrm{TP}_{\mathrm{TP}}$ & pAGM9121 & B3 & $\mathrm{TP}$ & BsaI, BpiI & $451 / 452$ & $\begin{array}{c}\text { Arabidopsis } \\
\text { genomic DNA }\end{array}$ \\
\hline$A t \mathrm{tTOCC}$ ТP & pAGM9121 & B3 & $\mathrm{TP}$ & BsaI, BpiI & $453 / 452$ & $\begin{array}{c}\text { Arabidopsis } \\
\text { genomic DNA }\end{array}$ \\
\hline synmRBS $_{\mathrm{TP}}$ & pAGM9121 & B3 & $\mathrm{TP}$ & BsaI, BpiI & $426 / 427$ & $\begin{array}{c}\mathrm{plCH} 78133 \\
\left(\mathrm{MoClo}^{1}\right)\end{array}$ \\
\hline$s_{n n c \mathrm{RBS}_{\mathrm{TP}}}$ & pAGM9121 & B3 & $\mathrm{TP}$ & BsaI, BpiI & $428 / 427$ & $\begin{array}{c}\text { plCH78133 } \\
\left(\mathrm{MoClo}^{1}\right)\end{array}$ \\
\hline$O s \mathrm{RBS} 1_{\mathrm{TP}}$ & pAGM9121 & B3 & $\mathrm{TP}$ & BsaI, BpiI & $759 / 760$ & $\begin{array}{c}\text { Oryza sativa } \\
\text { genomic DNA }\end{array}$ \\
\hline$A t \operatorname{Rec}_{\mathrm{TP}}$ & pAGM9121 & B3 & $\mathrm{TP}$ & BsaI, BpiI & $947 / 948$ & $\begin{array}{c}\text { Arabidopsis } \\
\text { genomic DNA }\end{array}$ \\
\hline nifH yco & pAGM9121 & B5 & CDS & BsaI, BpiI & $454 / 455$ & $\begin{array}{l}\text { Synthesis by } \\
\text { Proteogenix }\end{array}$ \\
\hline nifM yсо & pAGM9121 & B4-B5 & CDS & BsaI, BpiI & $456 / 457$ & $\begin{array}{l}\text { Synthesis by } \\
\text { GenScript }\end{array}$ \\
\hline nifS yco & pAGM9121 & B4-B5 & CDS & BsaI, BpiI & $\begin{array}{c}458 / 459 \\
(460)\end{array}$ & $\begin{array}{l}\text { Synthesis by } \\
\text { GenScript }\end{array}$ \\
\hline nif $U$ yco & pAGM9121 & B4-B5 & CDS & BsaI, BpiI & $461 / 462$ & $\begin{array}{l}\text { Synthesis by } \\
\text { GenScript }\end{array}$ \\
\hline nifH tsd & pAGM9121 & B5 & CDS & BsaI, BpiI & $463 / 502$ & $\begin{array}{l}\text { Synthesis by } \\
\text { NZYTech }\end{array}$ \\
\hline nifM tsd & pAGM9121 & B4-B5 & $\mathrm{CDS}$ & BsaI, BpiI & $465 / 503$ & $\begin{array}{l}\text { Synthesis by } \\
\text { NZYTech }\end{array}$ \\
\hline nifS tsd & pAGM9121 & B4-B5 & $\mathrm{CDS}$ & BsaI, BpiI & $467 / 504$ & $\begin{array}{l}\text { Synthesis by } \\
\text { NZYTech }\end{array}$ \\
\hline nif $U$ tsd & pAGM9121 & B4-B5 & CDS & BsaI, BpiI & $469 / 505$ & $\begin{array}{l}\text { Synthesis by } \\
\text { NZYTech }\end{array}$ \\
\hline Twin-Strep & pAGM9121 & B4 & Tag & BsaI, BpiI & $471 / 495$ & $\begin{array}{l}\text { Synthesis by } \\
\text { NZYTech }\end{array}$ \\
\hline CaMV p35S & pAGM9121 & A1-B2 & Promoter & BsaI, BpiI & $479 / 480$ & $\begin{array}{l}\mathrm{pICH} 51266 \\
\left(\mathrm{MoClo}^{1}\right)\end{array}$ \\
\hline CaMV p35S & pAGM9121 & A1-B3 & Promoter & BsaI, BpiI & $479 / 535$ & $\begin{array}{c}\mathrm{pICH} 51266 \\
\left(\mathrm{MoClo}^{1}\right)\end{array}$ \\
\hline $\begin{array}{l}\text { CaMV p35S } \\
\text { (short) }\end{array}$ & pAGM9121 & A1-B2 & Promoter & BsaI, BpiI & $812 / 480$ & $\begin{array}{l}\mathrm{pICH} 41388 \\
\left(\mathrm{MoClo}^{1}\right)\end{array}$ \\
\hline $\begin{array}{c}\text { 2x CaMV p35s + } \\
\text { 5'UTR TMV }\end{array}$ & pICH41295 & A1-B2 & Promoter & BsaI, BpiI & - & $\begin{array}{l}\mathrm{pICH} 51288 \\
\left(\mathrm{MoClo}^{1}\right)\end{array}$ \\
\hline nifH tsd & pAGM9121 & B5a & CDS & BsaI, BpiI & $463 / 578 / 579$ & $\begin{array}{l}\text { Synthesis by } \\
\text { NZYTech }\end{array}$ \\
\hline nif $M$ tsd & pAGM9121 & B4-B5a & $\mathrm{CDS}$ & BsaI, BpiI & $465 / 578 / 580$ & $\begin{array}{l}\text { Synthesis by } \\
\text { NZYTech }\end{array}$ \\
\hline
\end{tabular}




\begin{tabular}{|c|c|c|c|c|c|c|}
\hline nifS tsd & pAGM9121 & B4-B5a & CDS & BsaI, BpiI & $467 / 578 / 581$ & $\begin{array}{l}\text { Synthesis by } \\
\text { NZYTech }\end{array}$ \\
\hline nif $U$ tsd & pAGM9121 & B4-B5a & CDS & BsaI, BpiI & $469 / 578 / 582$ & $\begin{array}{l}\text { Synthesis by } \\
\text { NZYTech }\end{array}$ \\
\hline$t G F P$ & pAGM9121 & $\mathrm{B} 5 \mathrm{~b}$ & CDS & BsaI, BpiI & $576 / 577$ & $\begin{array}{l}\text { pICSL80005 } \\
\left(\mathrm{MoClo}^{1}\right)\end{array}$ \\
\hline mCherry & pAGM9121 & $\mathrm{B} 5 \mathrm{~b}$ & CDS & BsaI, BpiI & $714 / 715$ & $\begin{array}{c}\text { pICSL80007 } \\
\left(\mathrm{MoClo}^{1}\right)\end{array}$ \\
\hline mCherry & pAGM1301 & B5 & CDS & BsaI, BpiI & - & $\begin{array}{l}\text { pICSL50004 } \\
\left(\mathrm{MoClo}^{1}\right)\end{array}$ \\
\hline$B F P$ & pAGM9121 & B4-B5 & CDS & BsaI, BpiI & $712 / 713$ & GB0025² \\
\hline$e G F P$ & pICH41308 & B3-B5 & CDS & BsaI, BpiI & - & $\begin{array}{c}\mathrm{pICH} 41531 \\
\left(\mathrm{MoClo}^{1}\right)\end{array}$ \\
\hline$e G F P$ & pAGM9121 & B4-B5 & CDS & BsaI, BpiI & $621 / 622$ & $\begin{array}{l}\mathrm{pICH} 41531 \\
\left(\mathrm{MoClo}^{1}\right)\end{array}$ \\
\hline $\mathrm{T} 35 \mathrm{~s}$ & pICH41276 & B6-C1 & Terminator & BsaI, BpiI & - & $\begin{array}{c}\mathrm{pICH} 41414 \\
\left(\mathrm{MoClo}^{1}\right)\end{array}$ \\
\hline $\mathrm{pNOS}+\Omega$ & pICH41295 & A1-B2 & Promoter & BsaI, BpiI & - & $\begin{array}{c}\mathrm{pICH} 87633 \\
\left(\mathrm{MoClo}^{1}\right)\end{array}$ \\
\hline$p 19$ & pICH41308 & B3-B5 & $\begin{array}{l}\text { Silencing } \\
\text { inhibitor }\end{array}$ & BsaI, BpiI & - & $\begin{array}{c}\mathrm{pICH} 44022 \\
\left(\mathrm{MoClo}^{1}\right)\end{array}$ \\
\hline tNOS & pICH41276 & B6-C1 & Terminator & BsaI, BpiI & - & $\begin{array}{c}\mathrm{pICH} 41421 \\
\left(\mathrm{MoClo}^{1}\right)\end{array}$ \\
\hline pMaize Ubi1+1i & pAGM9121 & A1-B2 & Promoter & BsaI, BpiI & $595 / 596$ & $\begin{array}{l}\text { Synthesis by } \\
\text { GenScript }\end{array}$ \\
\hline pMaize Ubi1+1i & pAGM9121 & A1-B3 & Promoter & BsaI, BpiI & $595 / 758$ & $\begin{array}{l}\text { Synthesis by } \\
\text { GenScript }\end{array}$ \\
\hline pRice Actin1 & pAGM9121 & A1-B2 & Promoter & BsaI, BpiI & $\begin{array}{c}597 / 600 \\
(598 / 599)\end{array}$ & $\begin{array}{l}\text { Synthesis by } \\
\text { GenScript }\end{array}$ \\
\hline tADPGG & pAGM9121 & B6-C1 & Terminator & BsaI, BpiI & $629 / 630$ & $\begin{array}{l}\text { Synthesis by } \\
\text { GenScript }\end{array}$ \\
\hline HPT & pAGM9121 & B3-B5 & CDS & BsaI, BpiI & $765 / 766$ & $\begin{array}{c}\text { Paul } \\
\text { Christou's } 1 \mathrm{ab}^{3}\end{array}$ \\
\hline Twin-Strep rco & pAGM9121 & B4 & Tag & BsaI, BpiI & $771 / 772$ & $\begin{array}{l}\text { Synthesis by } \\
\text { GenScript }\end{array}$ \\
\hline nifH rco & pAGM9121 & B5 & CDS & BsaI, BpiI & $623 / 624$ & $\begin{array}{l}\text { Synthesis by } \\
\text { GenScript }\end{array}$ \\
\hline nifM rco & pAGM9121 & B4-B5 & CDS & BsaI, BpiI & $617 / 618$ & $\begin{array}{l}\text { Synthesis by } \\
\text { GenScript }\end{array}$ \\
\hline nifS rco & pAGM9121 & B4-B5 & CDS & BsaI, BpiI & $615 / 616$ & $\begin{array}{l}\text { Synthesis by } \\
\text { GenScript }\end{array}$ \\
\hline nif $U$ rco & pAGM9121 & B4-B5 & CDS & BsaI, BpiI & $613 / 614$ & $\begin{array}{l}\text { Synthesis by } \\
\text { GenScript }\end{array}$ \\
\hline
\end{tabular}




\begin{tabular}{|c|c|c|c|c|c|c|}
\hline nifH rco & pAGM9121 & B5a & CDS & BsaI, BpiI & $578 / 623 / 883$ & $\begin{array}{l}\text { Synthesis by } \\
\text { GenScript }\end{array}$ \\
\hline nif $M$ rco & pAGM9121 & B4-B5a & CDS & BsaI, BpiI & $578 / 617 / 884$ & $\begin{array}{l}\text { Synthesis by } \\
\text { GenScript }\end{array}$ \\
\hline nifS rco & pAGM9121 & B4-B5a & CDS & BsaI, BpiI & $578 / 615 / 886$ & $\begin{array}{l}\text { Synthesis by } \\
\text { GenScript }\end{array}$ \\
\hline nif $U$ rco & pAGM9121 & B4-B5a & CDS & BsaI, BpiI & $578 / 613 / 885$ & $\begin{array}{c}\text { Synthesis by } \\
\text { GenScript }\end{array}$ \\
\hline nifH & pAGM9121 & B5 & CDS & BsaI, BpiI & $\begin{array}{c}813 / 814 \\
(823 / 824 / 825 / \\
826 / 827 / 828)\end{array}$ & $\begin{array}{c}\text { Azotobacter } \\
\text { vinelandii } \\
\text { gDNA }\end{array}$ \\
\hline nifM & pAGM9121 & B4-B5 & CDS & BsaI, BpiI & $\begin{array}{c}815 / 816 \\
(829 / 830)\end{array}$ & $\begin{array}{c}\text { Azotobacter } \\
\text { vinelandii } \\
\text { gDNA }\end{array}$ \\
\hline nifS & pAGM9121 & B4-B5 & CDS & BsaI, BpiI & $\begin{array}{c}819 / 820 \\
(835 / 836 / 837 / \\
838)\end{array}$ & $\begin{array}{c}\text { Azotobacter } \\
\text { vinelandii } \\
\text { gDNA }\end{array}$ \\
\hline nifU & pAGM9121 & B4-B5 & CDS & BsaI, BpiI & $\begin{array}{c}817 / 818 \\
(831 / 832 / 833 / \\
834)\end{array}$ & $\begin{array}{c}\text { Azotobacter } \\
\text { vinelandii } \\
\text { gDNA }\end{array}$ \\
\hline nifH rsd & pAGM9121 & B5 & CDS & BsaI, BpiI & $\begin{array}{c}623 / 624 \\
(887 / 888 / 889 / \\
890 / 891 / 892)\end{array}$ & $\begin{array}{l}\text { Synthesis by } \\
\text { GenScript }\end{array}$ \\
\hline nif $M$ rsd & pAGM9121 & B4-B5 & CDS & BsaI, BpiI & $\begin{array}{c}617 / 618 \\
(893 / 894 / 895 / \\
896)\end{array}$ & $\begin{array}{l}\text { Synthesis by } \\
\text { GenScript }\end{array}$ \\
\hline$n i f S$ rsd & pAGM9121 & B4-B5 & CDS & BsaI, BpiI & $\begin{array}{c}615 / 616 \\
(897 / 898)\end{array}$ & $\begin{array}{c}\text { Synthesis by } \\
\text { GenScript }\end{array}$ \\
\hline nif $U$ rsd & pAGM9121 & B4-B5 & CDS & BsaI, BpiI & $\begin{array}{c}613 / 614 \\
(899 / 900 / 901 / \\
902 / 903 / 904 / 9 \\
05 / 906 / 907 / 90 \\
8 / 909 / 910)\end{array}$ & $\begin{array}{l}\text { Synthesis by } \\
\text { GenScript }\end{array}$ \\
\hline
\end{tabular}

Abbreviation: m: minimal; c: complete; yco: Yeast codon optimization; tsd: Tobacco synthetic design; rco: Rice codon optimization; rsd: Rice synthetic design. 
Supplemental Table 3. List of assembled Transcriptional Units.

\begin{tabular}{|c|c|c|}
\hline $\begin{array}{c}\text { Transcriptional Units } \\
\text { (Level 1) }\end{array}$ & Entry vector & Modular pieces used \\
\hline AtmAROA ${ }_{\mathrm{TP}}-n i f H_{\mathrm{tsd}}$ & pICH47732 & p35S::AtmAROA ${ }_{\mathrm{TP}}-$ Twin-Strep-nifH $H_{\mathrm{tsd}}-\mathrm{T} 35 \mathrm{~s}$ \\
\hline AtcAROA ${ }_{\mathrm{TP}}-n i f H_{\mathrm{tsd}}$ & pICH47732 & p35S::AtcAROA ${ }_{\mathrm{TP}}-$ Twin-Strep-nifH $H_{\mathrm{tsd}}-\mathrm{T} 35 \mathrm{~s}$ \\
\hline AtmBCCP1 $1_{\mathrm{TP}}-n i f H_{\mathrm{tsd}}$ & pICH47732 & $\mathrm{p} 35 \mathrm{~S}:: A t \mathrm{mBCCP} 1_{\mathrm{TP}}-\mathrm{Twin}-\mathrm{Strep}-n i f H_{\mathrm{tsd}}-\mathrm{T} 35 \mathrm{~s}$ \\
\hline$A t \mathrm{cBCCP} 1_{\mathrm{TP}}-n i f H_{\mathrm{tsd}}$ & pICH47732 & $\mathrm{p} 35 \mathrm{~S}:: A t c B C C P 1_{\mathrm{TP}}-\mathrm{Twin}-\mathrm{Strep}-n i f H_{\mathrm{tsd}}-\mathrm{T} 35 \mathrm{~s}$ \\
\hline AtmDNAJ8 $8_{\mathrm{TP}}-n i f H_{\mathrm{tsd}}$ & pICH47732 & p35S::AtmDNAJ8 $8_{\mathrm{TP}}-$ Twin-Strep-nifH $H_{\mathrm{tsd}}-\mathrm{T} 35 \mathrm{~s}$ \\
\hline$A t \mathrm{cDNAJ} 8_{\mathrm{TP}}-n i f H_{\mathrm{tsd}}$ & pICH47732 & p35S::AtcDNAJ8 $8_{\mathrm{TP}}-\mathrm{Tw}$ win-Strep-nifH $H_{\mathrm{tsd}}-\mathrm{T} 35 \mathrm{~s}$ \\
\hline AtmCAB6 $6_{\mathrm{TP}}-n i f H_{\mathrm{tsd}}$ & pICH47732 & $\mathrm{p} 35 \mathrm{~S}:: A t \mathrm{mCAB} 6_{\mathrm{TP}}-\mathrm{Twin}-\mathrm{Strep}-n i f H_{\mathrm{tsd}}-\mathrm{T} 35 \mathrm{~s}$ \\
\hline AtcCAB6$_{\mathrm{TP}}-n i f H_{\mathrm{tsd}}$ & pICH47732 & p35S::AtcCAB6 ${ }_{\mathrm{TP}}-$ Twin-Strep-nifH $H_{\mathrm{tsd}}-\mathrm{T} 35 \mathrm{~s}$ \\
\hline AtmGLTB2 $2_{\mathrm{TP}}-n i f H_{\mathrm{tsd}}$ & pICH47732 & p35S::AtmGLTB2 ${ }_{\mathrm{TP}}-$ Twin-Strep-nifH $H_{\mathrm{tsd}}-\mathrm{T} 35 \mathrm{~s}$ \\
\hline AtcGLTB $2_{\mathrm{TP}}-n i f H_{\mathrm{tsd}}$ & pICH47732 & $\mathrm{p} 35 \mathrm{~S}:: A t c G L T B 2_{\mathrm{TP}}-\mathrm{Twin}-\mathrm{Strep}-n i f H_{\mathrm{tsd}}-\mathrm{T} 35 \mathrm{~s}$ \\
\hline$N t \mathrm{mRBS}$ TP-nifH $H_{\mathrm{tsd}}$ & pICH47732 & $\mathrm{p} 35 \mathrm{~S}:: N t \mathrm{mRBS} \mathrm{TP}_{\mathrm{TP}}-\mathrm{Twin}-\mathrm{Strep}-n i f H_{\mathrm{tsd}}-\mathrm{T} 35 \mathrm{~s}$ \\
\hline$N t \mathrm{cRBS}$ TP-nifH $H_{\mathrm{tsd}}$ & pICH47732 & p35S::NtcRBS ${ }_{\mathrm{TP}}-$ Twin-Strep-nifH $H_{\mathrm{tsd}}-\mathrm{T} 35 \mathrm{~s}$ \\
\hline AtmRBS1 $1 \mathrm{~A}_{\mathrm{TP}}-n i f H_{\mathrm{tsd}}$ & pICH47732 & $\mathrm{p} 35 \mathrm{~S}::$ AtmRBS1 $\mathrm{A}_{\mathrm{TP}}-\mathrm{Twin}-\mathrm{Strep}-n i f H_{\mathrm{tsd}}-\mathrm{T} 35 \mathrm{~s}$ \\
\hline AtcRBS1 $\mathrm{A}_{\mathrm{TP}}-n i f H_{\mathrm{tsd}}$ & pICH47732 & $\mathrm{p} 35 \mathrm{~S}:: A t c R B S 1 \mathrm{~A}_{\mathrm{TP}}-\mathrm{Tw}$ win-Strep-nifH $H_{\mathrm{tsd}}-\mathrm{T} 35 \mathrm{~s}$ \\
\hline$P s \mathrm{mRBS} 2_{\mathrm{TP}}-n i f H_{\mathrm{tsd}}$ & pICH47732 & $\mathrm{p} 35 \mathrm{~S}:: P s m R B S 2_{\mathrm{TP}}-$ Twin-Strep-nifH $H_{\mathrm{tsd}}-\mathrm{T} 35 \mathrm{~s}$ \\
\hline$P s c R B S 2_{\mathrm{TP}}-n i f H_{\mathrm{tsd}}$ & pICH47732 & p35S::PscRBS2 ${ }_{\mathrm{TP}}-\mathrm{Twin}-\mathrm{Strep}-n i f H_{\mathrm{tsd}}-\mathrm{T} 35 \mathrm{~s}$ \\
\hline$A t \mathrm{mRCA} \mathrm{TP} n i f H_{\mathrm{tsd}}$ & pICH47732 & p35S::AtmRCA ${ }_{\mathrm{TP}}-\mathrm{Twin}-\mathrm{Strep}-n i f H_{\mathrm{tsd}}-\mathrm{T} 35 \mathrm{~s}$ \\
\hline$A t \mathrm{cRCA} \mathrm{TP}_{\mathrm{TP}} n i f H_{\mathrm{tsd}}$ & pICH47732 & p35S::AtcRCA ${ }_{\mathrm{TP}}-\mathrm{Tw}$ in-Strep-nifH $H_{\mathrm{tsd}}-\mathrm{T} 35 \mathrm{~s}$ \\
\hline$N t \mathrm{mSIR}$ TP-nifH $H_{\mathrm{tsd}}$ & pICH47732 & p35S::NtmSIR ${ }_{\mathrm{TP}}-$ Twin-Strep-nifH $H_{\mathrm{tsd}}-\mathrm{T} 35 \mathrm{~s}$ \\
\hline$N t \mathrm{cSIR}$ TP-nifH $H_{\mathrm{tsd}}$ & pICH47732 & p35S::NtcSIR ${ }_{\mathrm{TP}}-$ Twin-Strep-nifH $H_{\mathrm{tsd}}-\mathrm{T} 35 \mathrm{~s}$ \\
\hline
\end{tabular}




\begin{tabular}{|c|c|c|}
\hline$A t \mathrm{mTOCC} \mathrm{TP}_{\mathrm{TP}} n i f H_{\mathrm{tsd}}$ & pICH47732 & p35S::AtmTOCC ${ }_{\mathrm{TP}}-$ Twin-Strep-nifH $H_{\mathrm{tsd}}-\mathrm{T} 35 \mathrm{~s}$ \\
\hline$A t \mathrm{cTOCC} \mathrm{TP}_{\mathrm{TP}}-n i f H_{\mathrm{tsd}}$ & pICH47732 & p35S::AtcTOCC ${ }_{\text {TP-Twin-Strep-nifH }}$ tsd-T35s \\
\hline synmRBS $_{\mathrm{TP}}-n i f H_{\mathrm{tsd}}$ & pICH47732 & p35S::synmRBS ${ }_{\mathrm{TP}}-$ Twin-Strep-nifH $H_{\mathrm{tsd}}-\mathrm{T} 35 \mathrm{~s}$ \\
\hline$s y n c \mathrm{RBS}_{\mathrm{TP}}-n i f H_{\mathrm{tsd}}$ & pICH47732 & p35S::syncRBS ${ }_{\mathrm{TP}}-\mathrm{Twin}-\mathrm{Strep}-$ nifH $_{\mathrm{tsd}}-\mathrm{T} 35 \mathrm{~s}$ \\
\hline AtmAROA ${ }_{\mathrm{TP}}-n i f M_{\mathrm{tsd}}$ & pICH47742 & p35S::AtmAROA ${ }_{\mathrm{TP}}-n i f M_{\mathrm{tsd}}-\mathrm{T} 35 \mathrm{~s}$ \\
\hline $\operatorname{AtcAROA}_{\mathrm{TP}}-n i f M_{\mathrm{tsd}}$ & pICH47742 & p35S::AtcAROA ${ }_{\mathrm{TP}}-n i f M_{\mathrm{tsd}}-\mathrm{T} 35 \mathrm{~s}$ \\
\hline AtmBCCP1 $1_{\mathrm{TP}}-n i f M_{\mathrm{tsd}}$ & pICH47742 & $\mathrm{p} 35 \mathrm{~S}::$ AtmBCCP1 $_{\mathrm{TP}}-n i f M_{\mathrm{tsd}}-\mathrm{T} 35 \mathrm{~s}$ \\
\hline$A t \mathrm{cBCCP} 1_{\mathrm{TP}}-n i f M_{\mathrm{tsd}}$ & pICH47742 & $\mathrm{p} 35 \mathrm{~S}:: A t \mathrm{cBCCP} 1_{\mathrm{TP}}-n i f M_{\mathrm{tsd}}-\mathrm{T} 35 \mathrm{~s}$ \\
\hline AtmDNAJ $8_{\mathrm{TP}}-n i f M_{\mathrm{tsd}}$ & pICH47742 & $\mathrm{p} 35 \mathrm{~S}:: A t \mathrm{mDNAJ} 8_{\mathrm{TP}}-n i f M_{\mathrm{tsd}}-\mathrm{T} 35 \mathrm{~s}$ \\
\hline$A t \mathrm{cDNAJ} 8_{\mathrm{TP}}-n i f M_{\mathrm{tsd}}$ & pICH47742 & p35S::AtcDNAJ8 $8_{\mathrm{TP}}-n i f M_{\mathrm{tsd}}-\mathrm{T} 35 \mathrm{~s}$ \\
\hline AtmCAB6 $6_{\mathrm{TP}}-n i f M_{\mathrm{tsd}}$ & pICH47742 & $\mathrm{p} 35 \mathrm{~S}::$ AtmCAB6$_{\mathrm{TP}}-n i f M_{\mathrm{tsd}}-\mathrm{T} 35 \mathrm{~s}$ \\
\hline AtcCAB6 $6_{\mathrm{TP}}-n i f M_{\mathrm{tsd}}$ & pICH47742 & $\mathrm{p} 35 \mathrm{~S}::$ AtcCAB6$_{\mathrm{TP}}-n i f M_{\mathrm{tsd}}-\mathrm{T} 35 \mathrm{~s}$ \\
\hline AtmGLTB2 $2_{\mathrm{TP}}-n i f M_{\mathrm{tsd}}$ & pICH47742 & $\mathrm{p} 35 \mathrm{~S}:: A t \mathrm{mGLTB} 2_{\mathrm{TP}}-n i f M_{\mathrm{tsd}}-\mathrm{T} 35 \mathrm{~s}$ \\
\hline$A t c G L T B 2_{\mathrm{TP}}-n i f M_{\mathrm{tsd}}$ & pICH47742 & p35S::AtcGLTB2 $2_{\mathrm{TP}}-n i f M_{\mathrm{tsd}}-\mathrm{T} 35 \mathrm{~s}$ \\
\hline$N t \mathrm{mRBS} \mathrm{TP}_{\mathrm{P}}-n i f M_{\mathrm{tsd}}$ & pICH47742 & $\mathrm{p} 35 \mathrm{~S}:: N t \mathrm{mRBS} \mathrm{TP}_{\mathrm{TP}}-n i f M_{\mathrm{tsd}}-\mathrm{T} 35 \mathrm{~s}$ \\
\hline$N t \mathrm{cRBS} \mathrm{TP}_{\mathrm{TP}}-n i f M_{\mathrm{tsd}}$ & pICH47742 & $\mathrm{p} 35 \mathrm{~S}:: N t \mathrm{cRBS} \mathrm{TP}_{\mathrm{T}}-n i f M_{\mathrm{tsd}}-\mathrm{T} 35 \mathrm{~s}$ \\
\hline AtmRBS1 $\mathrm{A}_{\mathrm{TP}}-n i f M_{\mathrm{tsd}}$ & pICH47742 & $\mathrm{p} 35 \mathrm{~S}::$ AtmRBS1 $_{\mathrm{TP}}-n i f M_{\mathrm{tsd}}-\mathrm{T} 35 \mathrm{~s}$ \\
\hline$A t \mathrm{cRBS} 1 \mathrm{~A}_{\mathrm{TP}}-n i f M_{\mathrm{tsd}}$ & pICH47742 & p35S::AtcRBS1 $\mathrm{A}_{\mathrm{TP}}-n i f M_{\mathrm{tsd}}-\mathrm{T} 35 \mathrm{~s}$ \\
\hline$P s \mathrm{mRBS} 2_{\mathrm{TP}}-n i f M_{\mathrm{tsd}}$ & pICH47742 & $\mathrm{p} 35 \mathrm{~S}:: P s \mathrm{mRBS} 2_{\mathrm{TP}}-n i f M_{\mathrm{tsd}}-\mathrm{T} 35 \mathrm{~s}$ \\
\hline$P s \mathrm{cRBS} 2_{\mathrm{TP}}-n i f M_{\mathrm{tsd}}$ & pICH47742 & $\mathrm{p} 35 \mathrm{~S}:: P s \mathrm{cRBS} 2_{\mathrm{TP}}-n i f M_{\mathrm{tsd}}-\mathrm{T} 35 \mathrm{~s}$ \\
\hline$A t \mathrm{mRCA} \mathrm{TP}_{\mathrm{TP}}-n i f M_{\mathrm{tsd}}$ & pICH47742 & $\mathrm{p} 35 \mathrm{~S}:: A t \mathrm{mRCA} \mathrm{TP}_{\mathrm{TP}}-n i f M_{\mathrm{tsd}}-\mathrm{T} 35 \mathrm{~s}$ \\
\hline AtcRCA ${ }_{\mathrm{TP}}-n i f M_{\mathrm{tsd}}$ & pICH47742 & p35S::AtcRCA ${ }_{\mathrm{TP}}-n i f M_{\mathrm{tsd}}-\mathrm{T} 35 \mathrm{~s}$ \\
\hline
\end{tabular}




\begin{tabular}{|c|c|c|}
\hline$N t \mathrm{mSIR}$ TP-nifM $M_{\mathrm{tsd}}$ & pICH47742 & $\mathrm{p} 35 \mathrm{~S}:: N t \mathrm{mSIR} \mathrm{TP}_{\mathrm{TP}}-n i f M_{\mathrm{tsd}}-\mathrm{T} 35 \mathrm{~s}$ \\
\hline$N t \mathrm{cSIR} \mathrm{TP}_{\mathrm{P}} n i f M_{\mathrm{tsd}}$ & pICH47742 & p35S::NtcSIR ${ }_{\mathrm{TP}}-n i f M_{\mathrm{tsd}}-\mathrm{T} 35 \mathrm{~s}$ \\
\hline AtmTOCC ${ }_{\mathrm{TP}}-n i f M_{\mathrm{tsd}}$ & pICH47742 & $\mathrm{p} 35 \mathrm{~S}:: A t \mathrm{mTOCC} \mathrm{TP}_{\mathrm{TP}}-n i f M_{\mathrm{tsd}}-\mathrm{T} 35 \mathrm{~s}$ \\
\hline AtcTOCC ${ }_{\mathrm{TP}}-n i f M_{\mathrm{tsd}}$ & pICH47742 & p35S::AtcTOCC ${ }_{\mathrm{TP}}-n i f M_{\mathrm{tsd}}-\mathrm{T} 35 \mathrm{~s}$ \\
\hline synmRBS $_{\mathrm{TP}}-n i f M_{\mathrm{tsd}}$ & pICH47742 & p35S::synmRBS ${ }_{\mathrm{TP}}-n i f M_{\mathrm{tsd}}-\mathrm{T} 35 \mathrm{~s}$ \\
\hline$s y n c \mathrm{RBS}_{\mathrm{TP}}-n i f M_{\mathrm{tsd}}$ & pICH47742 & $\mathrm{p} 35 \mathrm{~S}:: s y n c R B \mathrm{~T}_{\mathrm{TP}}-n i f M_{\mathrm{tsd}}-\mathrm{T} 35 \mathrm{~s}$ \\
\hline$A t \mathrm{mAROA}$ TP-nif $U_{\mathrm{tsd}}$ & pICH47761 & $\mathrm{p} 35 \mathrm{~S}:: A t \mathrm{mAROA} \mathrm{TP}_{\mathrm{TP}}-n i f U_{\mathrm{tsd}}-\mathrm{T} 35 \mathrm{~s}$ \\
\hline AtcAROA $_{\mathrm{TP}}-n i f U_{\mathrm{tsd}}$ & pICH47761 & p35S::AtcAROA ${ }_{\mathrm{TP}}-n i f U_{\mathrm{tsd}}-\mathrm{T} 35 \mathrm{~s}$ \\
\hline AtmBCCP1 $1_{\mathrm{TP}}-n i f U_{\mathrm{tsd}}$ & pICH47761 & $\mathrm{p} 35 \mathrm{~S}:: A t \mathrm{mBCCP} 1_{\mathrm{TP}}-n i f U_{\mathrm{tsd}}-\mathrm{T} 35 \mathrm{~s}$ \\
\hline AtcBCCP1 $1_{\mathrm{TP}}-n i f U_{\mathrm{tsd}}$ & pICH47761 & $\mathrm{p} 35 \mathrm{~S}:: A t \mathrm{cBCCP} 1_{\mathrm{TP}}-n i f U_{\mathrm{tsd}}-\mathrm{T} 35 \mathrm{~s}$ \\
\hline AtmDNAJ8 $8_{\mathrm{TP}}-n i f U_{\mathrm{tsd}}$ & pICH47761 & $\mathrm{p} 35 \mathrm{~S}:: A t \mathrm{mDNAJ} 8_{\mathrm{TP}}-n i f U_{\mathrm{tsd}}-\mathrm{T} 35 \mathrm{~s}$ \\
\hline AtcDNAJ8 $8_{\mathrm{TP}}-n i f U_{\mathrm{tsd}}$ & pICH47761 & $\mathrm{p} 35 \mathrm{~S}:: A t \mathrm{cDNAJ} 8_{\mathrm{TP}}-n i f U_{\mathrm{tsd}}-\mathrm{T} 35 \mathrm{~s}$ \\
\hline AtmCAB6 ${ }_{\mathrm{TP}}-n i f U_{\mathrm{tsd}}$ & pICH47761 & $\mathrm{p} 35 \mathrm{~S}::$ AtmCAB $_{\mathrm{TP}}-n i f U_{\mathrm{tsd}}-\mathrm{T} 35 \mathrm{~s}$ \\
\hline AtcCAB6 ${ }_{\mathrm{TP}}-n i f U_{\mathrm{tsd}}$ & pICH47761 & p35S::AtcCAB6 ${ }_{\mathrm{TP}}-n i f U_{\mathrm{tsd}}-\mathrm{T} 35 \mathrm{~s}$ \\
\hline AtmGLTB2 $2_{\mathrm{TP}}-n i f U_{\mathrm{tsd}}$ & pICH47761 & $\mathrm{p} 35 \mathrm{~S}:: A t \mathrm{mGLTB} 2_{\mathrm{TP}}-n i f U_{\mathrm{tsd}}-\mathrm{T} 35 \mathrm{~s}$ \\
\hline AtcGLTB2 $2_{\mathrm{TP}}-n i f U_{\mathrm{tsd}}$ & pICH47761 & $\mathrm{p} 35 \mathrm{~S}:: A t c G L T B 2{ }_{\mathrm{TP}}-n i f U_{\mathrm{tsd}}-\mathrm{T} 35 \mathrm{~s}$ \\
\hline$N t \mathrm{mRBS}$ TP-nif $U_{\mathrm{tsd}}$ & pICH47761 & $\mathrm{p} 35 \mathrm{~S}:: N t \mathrm{mRBS} \mathrm{S}_{\mathrm{TP}}-n i f U_{\mathrm{tsd}}-\mathrm{T} 35 \mathrm{~s}$ \\
\hline$N t \mathrm{cRBS} \mathrm{TP}_{\mathrm{T}}-n i f U_{\mathrm{tsd}}$ & pICH47761 & $\mathrm{p} 35 \mathrm{~S}:: N t \mathrm{cRBS} \mathrm{T}_{\mathrm{TP}}-n i f U_{\mathrm{tsd}}-\mathrm{T} 35 \mathrm{~s}$ \\
\hline AtmRBS1 $1 \mathrm{~A}_{\mathrm{TP}}-n i f U_{\mathrm{tsd}}$ & pICH47761 & $\mathrm{p} 35 \mathrm{~S}:: A t \mathrm{mRBS} 1 \mathrm{~A}_{\mathrm{TP}}-n i f U_{\mathrm{tsd}}-\mathrm{T} 35 \mathrm{~s}$ \\
\hline$A t c R B S 1 \mathrm{~A}_{\mathrm{TP}}-n i f U_{\mathrm{tsd}}$ & pICH47761 & p35S::AtcRBS1 $\mathrm{A}_{\mathrm{TP}}-n i f U_{\mathrm{tsd}}-\mathrm{T} 35 \mathrm{~s}$ \\
\hline$P s \mathrm{mRBS} 2_{\mathrm{TP}}-n i f U_{\mathrm{tsd}}$ & pICH47761 & $\mathrm{p} 35 \mathrm{~S}:: P s \mathrm{mRBS} 2_{\mathrm{TP}}-n i f U_{\mathrm{tsd}}-\mathrm{T} 35 \mathrm{~s}$ \\
\hline$P s c R B S 2_{\mathrm{TP}}-n i f U_{\mathrm{tsd}}$ & pICH47761 & p35S::PscRBS2 $2_{\mathrm{TP}}-n i f U_{\mathrm{tsd}}-\mathrm{T} 35 \mathrm{~s}$ \\
\hline
\end{tabular}




\begin{tabular}{|c|c|c|}
\hline$A t \mathrm{mRCA} \mathrm{TP}_{\mathrm{TP}}-n i f U_{\mathrm{tsd}}$ & pICH47761 & $\mathrm{p} 35 \mathrm{~S}:: A t \mathrm{mRCA} \mathrm{TP}_{\mathrm{TP}}-n i f U_{\mathrm{tsd}}-\mathrm{T} 35 \mathrm{~s}$ \\
\hline AtcRCA $\mathrm{TP}_{\mathrm{TP}}-n i f U_{\mathrm{tsd}}$ & pICH47761 & p35S::AtcRCA $A_{\mathrm{TP}}-n i f U_{\mathrm{tsd}}-\mathrm{T} 35 \mathrm{~s}$ \\
\hline$N t \mathrm{mSIR} \mathrm{TP}_{\mathrm{P}}-n i f U_{\mathrm{tsd}}$ & pICH47761 & $\mathrm{p} 35 \mathrm{~S}:: N t \mathrm{mSIR}_{\mathrm{TP}}-n i f U_{\mathrm{tsd}}-\mathrm{T} 35 \mathrm{~s}$ \\
\hline$N t \mathrm{cSIR}$ TP-nif $U_{\text {tsd }}$ & pICH47761 & p35S::NtcSIR ${ }_{\mathrm{TP}}-n i f U_{\mathrm{tsd}}-\mathrm{T} 35 \mathrm{~s}$ \\
\hline$A t \mathrm{mTOCC}$ TP-nif $U_{\mathrm{tsd}}$ & pICH47761 & $\mathrm{p} 35 \mathrm{~S}:: A t \mathrm{mTOCC} \mathrm{TP}_{\mathrm{T}}-n i f U_{\mathrm{tsd}}-\mathrm{T} 35 \mathrm{~s}$ \\
\hline$A t \mathrm{cTOCC} \mathrm{TP}_{\mathrm{TP}}-n i f U_{\mathrm{tsd}}$ & pICH47761 & $\mathrm{p} 35 \mathrm{~S}:: A t \mathrm{cTOCC} \mathrm{TP}_{\mathrm{TP}}-n i f U_{\mathrm{tsd}}-\mathrm{T} 35 \mathrm{~s}$ \\
\hline synmRBS $_{\mathrm{TP}}-n i f U_{\mathrm{tsd}}$ & pICH47761 & p35S::synmRBS ${ }_{\mathrm{TP}}-n i f U_{\mathrm{tsd}}-\mathrm{T} 35 \mathrm{~s}$ \\
\hline$s y n c \mathrm{RBS}_{\mathrm{TP}}-n i f U_{\mathrm{tsd}}$ & pICH47761 & p35S::syncRBS ${ }_{\mathrm{TP}}-n i f U_{\mathrm{tsd}}-\mathrm{T} 35 \mathrm{~s}$ \\
\hline$A t \mathrm{mAROA} \mathrm{TP}_{\mathrm{TP}}-n i f S_{\mathrm{tsd}}$ & pICH47772 & p35S::AtmAROA ${ }_{\mathrm{TP}}-n i f S_{\mathrm{tsd}}-\mathrm{T} 35 \mathrm{~s}$ \\
\hline $\operatorname{AtcAROA}_{\mathrm{TP}}-n i f S_{\mathrm{tsd}}$ & pICH47772 & p35S::AtcAROA ${ }_{T P}-n i f S_{\text {tsd }}-T 35 \mathrm{~s}$ \\
\hline AtmBCCP1 $1_{\mathrm{TP}}-n i f S_{\mathrm{tsd}}$ & pICH47772 & p35S::AtmBCCP1 $1_{\mathrm{TP}}-n i f S_{\mathrm{tsd}}-\mathrm{T} 35 \mathrm{~s}$ \\
\hline$A t \mathrm{cBCCP} 1_{\mathrm{TP}}-n i f S_{\mathrm{tsd}}$ & pICH47772 & $\mathrm{p} 35 \mathrm{~S}:: A t \mathrm{cBCCP} 1_{\mathrm{TP}}-n i f S_{\mathrm{tsd}}-\mathrm{T} 35 \mathrm{~s}$ \\
\hline AtmDNAJ8 $8_{\mathrm{TP}}-n i f S_{\text {tsd }}$ & pICH47772 & p35S::AtmDNAJ8 $8_{\mathrm{TP}}-n i f S_{\mathrm{tsd}}-\mathrm{T} 35 \mathrm{~s}$ \\
\hline$A t \mathrm{cDNAJ} 8_{\mathrm{TP}}-n i f S_{\mathrm{tsd}}$ & pICH47772 & $\mathrm{p} 35 \mathrm{~S}::$ AtcDNAJ8$_{\mathrm{TP}}-n i f S_{\mathrm{tsd}}-\mathrm{T} 35 \mathrm{~s}$ \\
\hline AtmCAB6 $6_{\mathrm{TP}}-n i f S_{\mathrm{tsd}}$ & pICH47772 & $\mathrm{p} 35 \mathrm{~S}:: A t \mathrm{mCAB} 6_{\mathrm{TP}}-n i f S_{\mathrm{tsd}}-\mathrm{T} 35 \mathrm{~s}$ \\
\hline AtcCAB6 ${ }_{\mathrm{TP}}-n i f S_{\mathrm{tsd}}$ & pICH47772 & $\mathrm{p} 35 \mathrm{~S}::$ AtcCAB6$_{\mathrm{TP}}-n i f S_{\mathrm{tsd}}-\mathrm{T} 35 \mathrm{~s}$ \\
\hline AtmGLTB2 $2_{\mathrm{TP}}-n i f S_{\text {tsd }}$ & pICH47772 & p35S::AtmGLTB2 2 TP-nif $S_{\mathrm{tsd}}-\mathrm{T} 35 \mathrm{~s}$ \\
\hline$A t c G L T B 22_{\mathrm{TP}}-n i f S_{\mathrm{tsd}}$ & pICH47772 & $\mathrm{p} 35 \mathrm{~S}:: A t c G L T B 2_{\mathrm{TP}}-n i f S_{\mathrm{tsd}}-\mathrm{T} 35 \mathrm{~s}$ \\
\hline$N t \mathrm{mRBS} \mathrm{TP}_{\mathrm{TP}}-n i S_{\mathrm{tsd}}$ & pICH47772 & $\mathrm{p} 35 \mathrm{~S}:: N t \mathrm{mRBS} \mathrm{TP}_{\mathrm{TP}}-n i f S_{\mathrm{tsd}}-\mathrm{T} 35 \mathrm{~s}$ \\
\hline$N t \mathrm{cRBS}$ TP-nifS $S_{\mathrm{tsd}}$ & pICH47772 & p35S::NtcRBS ${ }_{\mathrm{TP}}-n i f S_{\mathrm{tsd}}-\mathrm{T} 35 \mathrm{~s}$ \\
\hline AtmRBS1 $1 \mathrm{~A}_{\mathrm{TP}}-n i f S_{\mathrm{tsd}}$ & pICH47772 & p35S::AtmRBS1A $\mathrm{TP}_{\mathrm{TP}}-n i f S_{\mathrm{tsd}}-\mathrm{T} 35 \mathrm{~s}$ \\
\hline$A t \mathrm{cRBS} 1 \mathrm{~A}_{\mathrm{TP}}-n i f S_{\mathrm{tsd}}$ & pICH47772 & p35S::AtcRBS1A $\mathrm{A}_{\mathrm{TP}}-n i f S_{\mathrm{tsd}}-\mathrm{T} 35 \mathrm{~s}$ \\
\hline
\end{tabular}




\begin{tabular}{|c|c|c|}
\hline$P s \mathrm{mRBS} 2_{\mathrm{TP}}-n i f S_{\mathrm{tsd}}$ & pICH47772 & $\mathrm{p} 35 \mathrm{~S}:: P s m R B S 2_{\mathrm{TP}}-n i f S_{\mathrm{tsd}}-\mathrm{T} 35 \mathrm{~s}$ \\
\hline$P s \mathrm{cRBS} 2_{\mathrm{TP}}-n i f S_{\mathrm{tsd}}$ & pICH47772 & p35S::PscRBS2 $2_{\mathrm{TP}}-n i f S_{\mathrm{tsd}}-\mathrm{T} 35 \mathrm{~s}$ \\
\hline AtmRCA ${ }_{\mathrm{TP}}-n i f S_{\mathrm{tsd}}$ & pICH47772 & $\mathrm{p} 35 \mathrm{~S}:: A t \mathrm{mRCA} \mathrm{T}_{\mathrm{TP}}-n i f S_{\mathrm{tsd}}-\mathrm{T} 35 \mathrm{~s}$ \\
\hline$A t \mathrm{cRCA}$ TP-nifS $S_{\mathrm{tsd}}$ & pICH47772 & p35S::AtcRCA $A_{\mathrm{TP}}-n i f S_{\mathrm{tsd}}-\mathrm{T} 35 \mathrm{~s}$ \\
\hline$N t \mathrm{mSIR}$ TP-nifS $S_{\mathrm{tsd}}$ & pICH47772 & $\mathrm{p} 35 \mathrm{~S}:: N t \mathrm{mSIR}$ TP-nifS $S_{\mathrm{tsd}}-\mathrm{T} 35 \mathrm{~s}$ \\
\hline$N t \mathrm{cSIR} \mathrm{TP}_{\mathrm{TP}}-n i f S_{\mathrm{tsd}}$ & pICH47772 & $\mathrm{p} 35 \mathrm{~S}:: N t \mathrm{cSIR} \mathrm{TP}_{\mathrm{T}-n i f S_{\mathrm{tsd}}-\mathrm{T} 35 \mathrm{~s}}$ \\
\hline AtmTOCC $\mathrm{TP}_{\mathrm{TP}} n i f S_{\mathrm{tsd}}$ & pICH47772 & $\mathrm{p} 35 \mathrm{~S}::$ AtmTOCC $\mathrm{TP}_{\mathrm{TP}}-n i f S_{\mathrm{tsd}}-\mathrm{T} 35 \mathrm{~s}$ \\
\hline$A t \mathrm{cTOCC} \mathrm{TP}_{\mathrm{TP}} n i f S_{\mathrm{tsd}}$ & pICH47772 & $\mathrm{p} 35 \mathrm{~S}:: A t \mathrm{cTOCC}$ TP-nifS $S_{\mathrm{tsd}}-\mathrm{T} 35 \mathrm{~s}$ \\
\hline synmRBS $_{\mathrm{TP}-n i f S_{\mathrm{tsd}}}$ & pICH47772 & $\mathrm{p} 35 \mathrm{~S}:: s y n \mathrm{mRBS} \mathrm{TP}_{\mathrm{T}}-n i f S_{\mathrm{tsd}}-\mathrm{T} 35 \mathrm{~s}$ \\
\hline$s_{y n c R B S}{ }_{\mathrm{TP}}-n i f S_{\mathrm{tsd}}$ & pICH47772 & p35S::syncRBS ${ }_{\mathrm{TP}-n i f S_{\mathrm{tsd}}-\mathrm{T} 35 \mathrm{~s}}$ \\
\hline cytonif $H_{\text {tsd }}$ & pICH47742 & p35S::Twin-Strep-nifH $H_{\mathrm{tsd}}-\mathrm{T} 35 \mathrm{~s}$ \\
\hline cytonif $M_{\mathrm{tsd}}$ & pICH47761 & $\mathrm{p} 35 \mathrm{~S}::$ nif $_{\mathrm{tsd}}-\mathrm{T} 35 \mathrm{~s}$ \\
\hline cytonif $S_{\mathrm{tsd}}$ & pICH47772 & p35S::nif $S_{\mathrm{tsd}}-\mathrm{T} 35 \mathrm{~s}$ \\
\hline cytonif $U_{\text {tsd }}$ & pICH47761 & p35S::nif $U_{\mathrm{tsd}}-\mathrm{T} 35 \mathrm{~s}$ \\
\hline $\begin{array}{c}A t \mathrm{mRCA} \mathrm{TP}_{\mathrm{TP}}-n i f H_{\mathrm{tsd}}- \\
t G F P\end{array}$ & pICH47732 & $\begin{array}{c}\mathrm{p} 35 \mathrm{~S}:: A t m R C A_{\mathrm{TP}}-\mathrm{Twin}_{\mathrm{S}} \mathrm{Strep}-n i f H_{\mathrm{tsd}}-t G F P- \\
\mathrm{T} 35 \mathrm{~s}\end{array}$ \\
\hline $\begin{array}{c}A t \mathrm{mBCCP} 1_{\mathrm{TP}-} n i f M_{\mathrm{tsd}^{-}} \\
t G F P\end{array}$ & pICH47742 & $\mathrm{p} 35 \mathrm{~S}:: A t \mathrm{mBCCP} 1_{\mathrm{TP}}-n i f M_{\mathrm{tsd}}-t G F P-\mathrm{T} 35 \mathrm{~s}$ \\
\hline $\begin{array}{l}\text { AtmBCCP1 } 1_{\mathrm{TP}-n i f M_{\mathrm{tsd}^{-}}} \\
\text {mCherry }\end{array}$ & pICH47742 & $\mathrm{p} 35 \mathrm{~S}::$ AtmBCCP1 $1_{\mathrm{TP}}-n i f M_{\mathrm{tsd}}-m$ Cherry-T35s \\
\hline $\begin{array}{c}A t \mathrm{mTOCC} \\
t G F P \\
t G P\end{array}$ & pICH47761 & $\mathrm{p} 35 \mathrm{~S}:: A t \mathrm{mTOCC} \mathrm{TP}_{\mathrm{TP}}-n i f U_{\mathrm{tsd}}-t G F P-\mathrm{T} 35 \mathrm{~s}$ \\
\hline $\begin{array}{c}A t \mathrm{mCAB} 6_{\mathrm{TP}}-n i f S_{\mathrm{tsd}^{-}} \\
t G F P\end{array}$ & pICH47772 & p35S::AtmCAB6 ${ }_{\mathrm{TP}}-n i f S_{\mathrm{tsd}}-t G F P-\mathrm{T} 35 \mathrm{~s}$ \\
\hline$A t \mathrm{mRCA} \mathrm{TP}_{\mathrm{TP}} n i f H_{\mathrm{yco}}$ & pICH47732 & p35S::AtmRCA ${ }_{\mathrm{TP}}-$ Twin-Strep-nifH $H_{\mathrm{yco}}-\mathrm{T} 35 \mathrm{~s}$ \\
\hline AtmBCCP1 $1_{\mathrm{TP}}-n i f M_{y c o}$ & pICH47742 & $\mathrm{p} 35 \mathrm{~S}:: A t \mathrm{mBCCP} 1_{\mathrm{TP}}-n i f M_{\mathrm{yco}}-\mathrm{T} 35 \mathrm{~s}$ \\
\hline AtmTOCC $\mathrm{TP}_{\mathrm{T}}-n i f U_{\mathrm{yco}}$ & pICH47761 & $\mathrm{p} 35 \mathrm{~S}:: A t \mathrm{mTOCC} \mathrm{TP}_{\mathrm{TP}}-n i f U_{\mathrm{yco}}-\mathrm{T} 35 \mathrm{~s}$ \\
\hline
\end{tabular}




\begin{tabular}{|c|c|c|}
\hline AtmCAB6тр-nifS $S_{\text {усо }}$ & pICH47772 & p35S::AtmCAB6 ${ }_{\mathrm{TP}}-n i f S_{\mathrm{yco}}-\mathrm{T} 35 \mathrm{~s}$ \\
\hline$s_{y n c} \mathrm{RBS}_{\mathrm{TP}}-B F P$ & pICH47781 & p35S::syncRBS ${ }_{\mathrm{TP}}-B F P-\mathrm{T} 35 \mathrm{~s}$ \\
\hline$A t \operatorname{Rec} \mathrm{A}_{\mathrm{TP}}-\mathrm{mCherry}$ & pICH47742 & 2x p35S::AtRec $\mathrm{A}_{\mathrm{TP}}-\mathrm{mCherry}-\mathrm{T} 35 \mathrm{~s}$ \\
\hline P19 & pICH47751 & $\mathrm{pNOS}+\Omega:: p 19-\mathrm{tNOS}$ \\
\hline cytoe $G F P$ & pICH47781 & p35S::eGFP-T35s \\
\hline cytoeGFP & pICH47732 & p35S::eGFP-T35s \\
\hline$A_{t \mathrm{mRCA}} \mathrm{TP}_{\mathrm{T}} e G F P$ & pICH47732 & $2 \times \mathrm{p} 35 \mathrm{~S}:: A t \mathrm{mRCA} \mathrm{TP}_{\mathrm{TP}} e G F P$-tNOS \\
\hline AtmBCCP1 $1_{\mathrm{TP}}-e G F P$ & pICH47732 & $2 \times \mathrm{p} 35 \mathrm{~S}:: A t \mathrm{mBCCP} 1_{\mathrm{TP}}-e G F P$-tNOS \\
\hline$A t \mathrm{mTOCC} \mathrm{TP}_{\mathrm{TP}}-e G F P$ & pICH47732 & $2 \times \mathrm{p} 35 \mathrm{~S}:: A t \mathrm{mTOCC} \mathrm{CP}_{\mathrm{TP}}-e G F P-\mathrm{tNOS}$ \\
\hline$A t \mathrm{mCAB} 6 \mathrm{TP}-e G F P$ & pICH47732 & $2 \mathrm{x} 35 \mathrm{~S}:: A t \mathrm{mCAB} 6_{\mathrm{TP}}-e G F P-\mathrm{tNOS}$ \\
\hline$A t c G L T B 2$ TP-eGFP & pICH47732 & 2x p35S::AtcGLTB2 $2_{\mathrm{TP}}-e G F P-\mathrm{tNOS}$ \\
\hline OsRBS1 $1_{\mathrm{TP}-} e G F P$ & pICH47732 & $2 \times \mathrm{p} 35 \mathrm{~S}:: \mathrm{OsRBS} 1_{\mathrm{TP}-e G F P-\mathrm{tNOS}}$ \\
\hline pUC57-L1P1 & pUC57-AmpR & Cloning cassette pICH47732 \\
\hline pUC57-L1P2 & pUC57-AmpR & Cloning cassette pICH47742 \\
\hline pUC57-L1P3 & pUC57-AmpR & Cloning cassette pICH47751 \\
\hline pUC57-L1P4 & pUC57-AmpR & Cloning cassette pICH47761 \\
\hline pUC57-L1P5 & pUC57-AmpR & Cloning cassette pICH47772 \\
\hline pUC57-L1P6 & pUC57-AmpR & Cloning cassette pICH47781 \\
\hline HPТ & pUC57-L1P3 & P35s (short)::HPT-tNOS \\
\hline cytoe $G F P$ & pUC57-L1P1 & pMaize Ubi1 $+1:: e G F P$-tNOS \\
\hline cytoeGFP & pUC57-L1P3 & pMaize Ubi1 +1::eGFP-tNOS \\
\hline AtmRCA $\mathrm{TP}_{\mathrm{TP}} e G F P$ & pUC57-L1P1 & pMaize Ubi1 + 1::AtmRCA ${ }_{\mathrm{TP}} e G F P$-tNOS \\
\hline
\end{tabular}




\begin{tabular}{|c|c|c|}
\hline AtmBCCP1 $1_{\mathrm{TP}-e G F P}$ & pUC57-L1P1 & pMaize Ubi1 $+1:: A t m B C C P 1_{\mathrm{TP}-e G F P-t N O S}$ \\
\hline$A t \mathrm{mTOCC}$ TP-eGFP & pUC57-L1P1 & pMaize Ubil $+1:: A t m T O C C_{\text {TP }} e G F P$-tNOS \\
\hline$A t \mathrm{mCAB} 6_{\mathrm{TP}}-e G F P$ & pUC57-L1P1 & pMaize Ubi1 +1::AtmCAB6 ${ }_{\mathrm{TP}}-e G F P$-tNOS \\
\hline AtcGLTB2 $2_{\mathrm{TP}}-e G F P$ & pUC57-L1P1 & pMaize Ubi1 +1::AtcGLTB2 $2_{\mathrm{TP}}-e G F P$-tNOS \\
\hline OsRBS1 $1_{\mathrm{TP}-e G F P}$ & pUC57-L1P1 & pMaize Ubi1 + 1::OsRBS1 $1_{\mathrm{TP}}-e G F$-tNOS \\
\hline cytonif $H_{\text {rco }}$ & pUC57-L1P1 & $\begin{array}{c}\text { pMaize Ubil }+1:: \text { Twin-Strep rco-nifH } H_{\mathrm{rco}^{-}} \\
\text {tNOS }\end{array}$ \\
\hline cytonif $M_{\mathrm{rco}}$ & pUC57-L1P2 & pMaize Ubi1 $+1::$ nif $M_{\mathrm{rco}}$-tNOS \\
\hline cytonif $S_{\text {rco }}$ & pUC57-L1P4 & pMaize Ubi1 +1::nifS $S_{\text {rco }}$-tNOS \\
\hline cytonif $U_{\text {rco }}$ & pUC57-L1P5 & pMaize Ubi1 $+1:: n i f U_{\text {rco-tNOS }}$ \\
\hline AtmRCA $_{\mathrm{TP}}-n i f H_{\text {rco }}$ & pUC57-L1P1 & $\begin{array}{c}\text { pMaize Ubil }+1:: \text { AtmRCA }_{\mathrm{TP}} \text {-Twin-Strep } \mathrm{rco}^{-} \\
n i f H_{\mathrm{rco}}-\mathrm{tNOS}\end{array}$ \\
\hline AtmBCCP1 $1_{\mathrm{TP}}-n i f M_{\mathrm{rco}}$ & pUC57-L1P2 & pMaize Ubi1 +1::AtmBCCP1 $1_{\mathrm{TP}}-n i f M_{\mathrm{rco}}-\mathrm{tNOS}$ \\
\hline AtmTOCC $\mathrm{TP}_{\mathrm{TP}}-n i f U_{\mathrm{rco}}$ & pUC57-L1P4 & pMaize Ubi1 $+1:: A t m T O C C_{\mathrm{TP}}-n i f U_{\mathrm{rco}}-\mathrm{tNOS}$ \\
\hline AtmCAB6 $6_{\mathrm{TP}}-n i f S_{\text {rco }}$ & pUC57-L1P5 & pMaize Ubil $+1::$ AtmCAB6 ${ }_{\mathrm{TP}}-n i f S_{\mathrm{rco}}$-tNOS \\
\hline $\begin{array}{l}\text { AtmBCCP1 } 1_{\mathrm{TP}}-n i f M_{\mathrm{rco}} \\
\text { (b) }\end{array}$ & pUC57-L1P2 & 2x p35S::AtmBCCP1 $1_{\mathrm{TP}}-n i f M_{\mathrm{rco}}-\mathrm{tNOS}$ \\
\hline $\begin{array}{l}A t \mathrm{mTOCC} \\
\text { (b) }\end{array}$ & pUC57-L1P4 & $2 \times \mathrm{p} 35 \mathrm{~S}:: A t \mathrm{mTOCC} \mathrm{TP}_{\mathrm{TP}}-n i f U_{\mathrm{rco}}-\mathrm{tADPGG}$ \\
\hline$A_{t m C A B} 6_{\mathrm{TP}}-n i f S_{\text {rco }}(\mathrm{b})$ & pUC57-L1P5 & pRice Actin $1:: A t \mathrm{mCAB} 6_{\mathrm{TP}}-n i f S_{\mathrm{rco}}$-tADPGG \\
\hline $\begin{array}{l}\text { AtmRCA }{ }_{\mathrm{TP}}-n i f H_{\mathrm{rco}^{-}} \\
t G F P\end{array}$ & pICH47732 & $\begin{array}{c}\text { pMaize Ubil }+1:: \text { AtmRCA } \mathrm{TP}_{\mathrm{TP}} \text {-Twin-Strep } \mathrm{rco}^{-} \\
\text {nifH }_{\mathrm{rco}}-t G F P-\mathrm{tNOS}\end{array}$ \\
\hline $\begin{array}{l}\text { AtmBCCP1 } 1_{\mathrm{TP}-} \text { nif } M_{\mathrm{rco}^{-}} \\
\text {mCherry }\end{array}$ & pICH47742 & $\begin{array}{l}\text { pMaize Ubi1 }+1:: \text { AtmBCCP1 } 1_{\mathrm{TP}-n i f M_{\mathrm{rco}^{-}}} \\
\text {mCherry- tNOS }\end{array}$ \\
\hline AtmRCA $_{\mathrm{TP}}-n i f H_{\mathrm{nco}}$ & pUC57-L1P1 & $\begin{array}{c}\text { pMaize Ubi1 }+1:: \text { AtmRCA }_{\mathrm{TP}} \text {-Twin-Strep } \text { nco- }^{-} \\
n i f H_{\mathrm{nco}}-\mathrm{tNOS}\end{array}$ \\
\hline AtmBCCP1 $1_{\mathrm{TP}}-n i f M_{\mathrm{nco}}$ & pUC57-L1P2 & pMaize Ubi1 +1::AtmBCCP1 $1_{\mathrm{TP}}-n i f M_{\mathrm{nco}}$-tNOS \\
\hline AtmTOCC $\mathrm{TP}_{\mathrm{TP}}-n i f U_{\mathrm{nco}}$ & pUC57-L1P4 & pMaize Ubi $1+1:: A t m T O C C_{\mathrm{TP}}-n i f U_{\mathrm{nco}}-\mathrm{tNOS}$ \\
\hline AtmCAB6 ${ }_{\mathrm{TP}}-n i f S_{\mathrm{nco}}$ & pUC57-L1P5 & pMaize Ubi1 +1::AtmCAB6 ${ }_{\mathrm{TP}}-n i f S_{\mathrm{nco}}$-tNOS \\
\hline
\end{tabular}




\begin{tabular}{|c|c|c|}
\hline AtmRCA ${ }_{\mathrm{TP}}-n i f H_{\mathrm{yco}}$ & pUC57-L1P1 & $\begin{array}{c}\text { pMaize Ubi1 }+1:: \text { AtmRCA }_{\mathrm{TP}} \text {-Twin-Strep }{ }_{\mathrm{yco}^{-}} \\
\text {nifH } H_{\mathrm{yco}}-\mathrm{tNOS}\end{array}$ \\
\hline AtmBCCP1 $1_{\mathrm{TP}}-n i f M_{\mathrm{yco}}$ & pUC57-L1P2 & pMaize Ubi1 $+1:: A t \mathrm{mBCCP} 1_{\mathrm{TP}}-n i f M_{\mathrm{yco}}-\mathrm{tNOS}$ \\
\hline AtmTOCC $\mathrm{TP}_{\mathrm{TP}}-n i f U_{\mathrm{yco}}$ & pUC57-L1P4 & pMaize Ubi1 +1::AtmTOCC $\mathrm{TP}_{\mathrm{TP}}-n i f U_{\mathrm{yco}}-\mathrm{tNOS}$ \\
\hline AtmCAB6 & pUC57-L1P5 & pMaize Ubi1 +1::AtmCAB6 ${ }_{\mathrm{TP}}-n i f S_{\mathrm{yco}}$-tNOS \\
\hline AtmRCA ${ }_{\mathrm{TP}}-n i f H_{\mathrm{tsd}}$ & pUC57-L1P1 & $\begin{array}{c}\text { pMaize Ubi1 }+1:: A t \mathrm{mRCA}_{\mathrm{TP}}-\text { Twin-Strep }_{\mathrm{tsd}^{-}} \\
\text {nifH } \mathrm{tsd}_{\mathrm{ts}}-\mathrm{t} \mathrm{NOS}\end{array}$ \\
\hline AtmBCCP1 $1_{\mathrm{TP}}-n i f M_{\mathrm{tsd}}$ & pUC57-L1P2 & pMaize Ubi1 $+1::$ AtmBCCP1 $1_{\mathrm{TP}}-n i f M_{\mathrm{tsd}}-\mathrm{tNOS}$ \\
\hline$A t \mathrm{mTOCC} \mathrm{TP}_{\mathrm{TP}}-n i f U_{\mathrm{tsd}}$ & pUC57-L1P4 & pMaize Ubi1 $+1:: A t m T O C C_{\text {TP- }} n i f U_{\text {tsd }}-\mathrm{tNOS}$ \\
\hline$A t \mathrm{mCAB} 6_{\mathrm{TP}}-n i f S_{\mathrm{tsd}}$ & pUC57-L1P5 & pMaize Ubi1 $+1:: A t m C A B 6_{\mathrm{TP}}-n i f S_{\mathrm{tsd}}-\mathrm{tNOS}$ \\
\hline$A t \mathrm{mRCA} \mathrm{TP}_{\mathrm{TP}} n i f H_{\mathrm{rsd}}$ & pUC57-L1P1 & $\begin{array}{c}\text { pMaize Ubi } 1+1:: A t m R C A_{\mathrm{TP}}-\text { Twin-Strep }_{\mathrm{rsd}}- \\
\text { nif } H_{\mathrm{rsd}}-\mathrm{tNOS}\end{array}$ \\
\hline AtmBCCP1 $1_{\mathrm{TP}}-n i f M_{\mathrm{rsd}}$ & pUC57-L1P2 & pMaize Ubi1 $+1:: A t m B C C P 1_{\mathrm{TP}}-n i f M_{\mathrm{rdd}}-\mathrm{tNOS}$ \\
\hline$A t \mathrm{mTOCC} \mathrm{TP}_{\mathrm{TP}} n i f U_{\mathrm{rsd}}$ & pUC57-L1P4 & pMaize Ubi $1+1:: A t m T O C C_{\mathrm{TP}}-n i f U_{\mathrm{rsd}}-\mathrm{tNOS}$ \\
\hline AtmCAB6 $6_{\mathrm{TP}}-n i f S_{\mathrm{rsd}}$ & pUC57-L1P5 & pMaize Ubi1 $+1::$ AtmCAB6 $_{\mathrm{TP}}-n i f S_{\mathrm{rdd}}-\mathrm{tNOS}$ \\
\hline
\end{tabular}

Abbreviation: m: minimal; c: complete; yco: Yeast codon optimization; tsd: Tobacco synthetic design; rco: Rice codon optimization; rsd: Rice synthetic design. 
Supplemental Table 4. List of assembled multigenic constructions.

\begin{tabular}{|c|c|c|}
\hline $\begin{array}{l}\text { Multigenic constructions } \\
\text { (Level 2) }\end{array}$ & Entry vector & Transcriptional Units used \\
\hline mChloro-nifH-nifM-nifU-nifS-P19 & pAGM4673 & $\begin{array}{c}\text { AtmRCA }_{\mathrm{TP}}-n i f H_{\mathrm{tsd}}+A t \mathrm{mBCCP} 1_{\mathrm{TP}}-n i f M_{\mathrm{tsd}}+ \\
\text { AtmTOCC }_{\mathrm{TP}}-n i f U_{\mathrm{tsd}}+A t \mathrm{mCAB} 6_{\mathrm{TP}}-n i f S_{\mathrm{tsd}}+ \\
\text { P19 }\end{array}$ \\
\hline mChloro-nifH-nifM-P19 & pAGM4673 & $\begin{array}{c}A t \mathrm{mRCA} \mathrm{TP}_{\mathrm{TP}} n i f H_{\mathrm{tsd}}+A t \mathrm{mBCCP} 1_{\mathrm{TP}}-n i f M_{\mathrm{tsd}}+ \\
\mathrm{P} 19\end{array}$ \\
\hline mChloro-nifH(tGFP)-nifM(mCherry)-P19 & pAGM4673 & $\begin{array}{c}A t \mathrm{mRCA} \\
\text { nif }-n i f H_{\mathrm{tsd}}-m \text { Cherry } \\
\text { ts }-\mathrm{P} 19\end{array}$ \\
\hline mChloro-nifH(tGFP)-cChloro-BFP-P19 & pAGM4673 & $\begin{array}{c}A t \mathrm{mRCA} \mathrm{TP}_{\mathrm{TP}}-n i f H_{\mathrm{tsd}}-t G F P+s y n \mathrm{cRBS} \mathrm{TP}_{\mathrm{TP}}-B F P \\
+\mathrm{P} 19\end{array}$ \\
\hline mChloro-nifM(tGFP)-cChloro-BFP-P19 & pAGM4673 & $\begin{array}{c}A t \mathrm{mBCCP} 1_{\mathrm{TP}-} n i f M_{\mathrm{tsd}} t G F P+s y n c \mathrm{RBS}_{\mathrm{TP}}- \\
B F P+\mathrm{P} 19\end{array}$ \\
\hline mChloro-nifU(tGFP)-cChloro-BFP-P19 & pAGM4673 & $\begin{array}{c}A t \mathrm{mTOCC} \\
\mathrm{TP}-n i f U_{\mathrm{tsd}}-t G F P+s y n \mathrm{cRBS}_{\mathrm{TP}}-B F P \\
+\mathrm{P} 19\end{array}$ \\
\hline mChloro-nifS(tGFP)-cChloro-BFP-P19 & pAGM4673 & $\begin{array}{c}A t \mathrm{mCAB} 6 \mathrm{TP}-n i f S_{\mathrm{tsd}}-t G F P+s y n \mathrm{cRBS} \\
+\mathrm{P} 19\end{array}$ \\
\hline mChloro-nifH $H_{\text {tsd }}$-cytoe $G F P$-P19 & pAGM4673 & $\begin{array}{c}\text { AtmRCA }{ }_{\mathrm{TP}}-\text { Twin-Strep- } n \text { if } H_{\mathrm{tsd}}+\text { cytoeGFP }+ \\
\text { P19 }\end{array}$ \\
\hline mChloro-nif $M_{\text {tsd }}$-cytoe $G F P$-P19 & pAGM4673 & AtmBCCP1 $1_{\mathrm{TP}}-n i f M_{\mathrm{tsd}}+$ cytoeGFP $+\mathrm{P} 19$ \\
\hline mChloro-nif $U_{\text {tsd-cytoe } G F P-\mathrm{P} 19}$ & pAGM4673 & AtmTOCC $\mathrm{TP}_{\mathrm{TP}} n i f U_{\mathrm{tsd}}+$ cytoeGFP $+\mathrm{P} 19$ \\
\hline mChloro-nif $S_{\text {tsd-cytoe } G F P-\mathrm{P} 19}$ & pAGM4673 & AtmCAB6 $6_{\mathrm{TP}}-n i f S_{\mathrm{tsd}}+$ cytoeGFP $+\mathrm{P} 19$ \\
\hline mChloro-nifH $H_{\mathrm{yco}}-$ cytoe $G F P-\mathrm{P} 19$ & pAGM4673 & $\begin{array}{c}\text { AtmRCA }{ }_{\mathrm{TP}}-\text { Twin-Strep- } n i f H_{\mathrm{yco}}+\text { cytoeGFP }+ \\
\text { P19 }\end{array}$ \\
\hline mChloro-nifM $M_{\mathrm{yco}}-$ cytoe $G F P$-P19 & pAGM4673 & AtmBCCP1 $1_{\mathrm{TP}}-n i f M_{\mathrm{yco}}+$ cytoeGFP $+\mathrm{P} 19$ \\
\hline mChloro-nif $U_{\text {yсо-cytoe} G F P-\mathrm{P} 19}$ & pAGM4673 & AtmTOCC $\mathrm{TP}_{\mathrm{TP}}-n i f U_{\mathrm{yco}}+$ cytoeGFP $+\mathrm{P} 19$ \\
\hline mChloro-nif $S_{\text {yco-cytoe } G F P-\mathrm{P} 19}$ & pAGM4673 & AtmCAB6 $6_{\mathrm{TP}}-n i f S_{\mathrm{yco}}+$ cytoeGFP $+\mathrm{P} 19$ \\
\hline $\begin{array}{c}\text { mChloro-nifH-nifM-nifU-nifS-cytoeGFP- } \\
\text { P19 }\end{array}$ & pAGM4673 & $\begin{array}{c}\text { AtmRCA }_{\mathrm{TP}}-n i f H_{\mathrm{tsd}}+A t \mathrm{mBCCP} 1_{\mathrm{TP}}-n i f M_{\mathrm{tsd}}+ \\
\text { AtmTOCC }_{\mathrm{TP}}-n i f U_{\mathrm{tsd}}+A t \mathrm{mCAB} 6_{\mathrm{TP}}-n i f S_{\mathrm{tsd}}+ \\
\text { cytoeGFP}+\mathrm{P} 19\end{array}$ \\
\hline cytoeGFP-P19 & pAGM4673 & cytoe $G F P+\mathrm{P} 19$ \\
\hline$A t \mathrm{mRCA} \mathrm{TP}_{\mathrm{TP}} e G F P-\mathrm{P} 19$ & pAGM4673 & $A t \mathrm{mRCA} \mathrm{TP}_{\mathrm{TP}} e G F P+\mathrm{P} 19$ \\
\hline AtmBCCP1 $1_{\mathrm{TP}}-e G F P-\mathrm{P} 19$ & pAGM4673 & AtmBCCP1 $1_{\mathrm{TP}}-e G F P+\mathrm{P} 19$ \\
\hline AtmTOCC $\mathrm{TP}_{\mathrm{TP}}-e G F P-\mathrm{P} 19$ & pAGM4673 & AtmTOCC $\mathrm{TP}_{\mathrm{TP}} e G F P+\mathrm{P} 19$ \\
\hline
\end{tabular}




\begin{tabular}{|c|c|c|}
\hline AtmCAB6 & pAGM4673 & AtmCAB6тр-eGFP + $\mathrm{P} 19$ \\
\hline AtcGLTB2 2 TP-eGFP-P19 & pAGM4673 & $A t \mathrm{cGLTB} 2_{\mathrm{TP}}-e G F P+\mathrm{P} 19$ \\
\hline OsRBS1 $1_{\mathrm{TP}}-e G F P-\mathrm{P} 19$ & pAGM4673 & OsRBS1 $1_{\mathrm{TP}-e G F P}+\mathrm{P} 19$ \\
\hline cytoe $G F P+A t \operatorname{Rec} \mathrm{A}_{\mathrm{TP}}-\mathrm{mCherry}$ & pAGM4673 & cytoe $G F P+\mathrm{P} 19+A t \operatorname{Rec} \mathrm{A}_{\mathrm{TP}}-\mathrm{mCherry}$ \\
\hline$A t \mathrm{mRCA} \mathrm{AP}_{\mathrm{TP}}-e G F+A t \operatorname{Rec} \mathrm{A}_{\mathrm{TP}}-\mathrm{mCherry}$ & pAGM4673 & $\begin{array}{c}A t \mathrm{mRCA} \mathrm{TP}_{\mathrm{P}} e G F P+\mathrm{P} 19+A t \mathrm{Rec}_{\mathrm{TP}}- \\
\text { mCherry }\end{array}$ \\
\hline$A t \mathrm{mBCCP} 1_{\mathrm{TP}} e G F P+A t \operatorname{Rec} \mathrm{A}_{\mathrm{TP}}-\mathrm{mCherry}$ & pAGM4673 & $\begin{array}{c}A t \mathrm{mBCCP} 1_{\mathrm{TP}}-e G F P+\mathrm{P} 19+A t \operatorname{Rec} \mathrm{A}_{\mathrm{TP}}- \\
\text { mCherry }\end{array}$ \\
\hline AtmTOCTP- $e G F P+A t$ RecA $\mathrm{AP}_{\mathrm{TP}}-\mathrm{mCherry}$ & pAGM4673 & $\begin{array}{c}A t \mathrm{mTOCTP}-e G F P+\mathrm{P} 19+A t \mathrm{Rec}_{\mathrm{TP}}- \\
\text { mCherry }\end{array}$ \\
\hline$A t \mathrm{mCAB} 6 \mathrm{TP}-e G F P+A t$ RecA $\mathrm{A}_{\mathrm{TP}}-\mathrm{mCherry}$ & pAGM4673 & $\begin{array}{c}A t \mathrm{mCAB} 6_{\mathrm{TP}}-e G F P+\mathrm{P} 19+A t \mathrm{Rec}_{\mathrm{TP}}- \\
\text { mCherry }\end{array}$ \\
\hline$A t \mathrm{cGLTB} 2_{\mathrm{TP}}-e G F P+A t \operatorname{Rec} \mathrm{A}_{\mathrm{TP}}-\mathrm{mCherry}$ & pAGM4673 & $\begin{array}{c}A t \mathrm{cGLTB} 2 \mathrm{TP}-e G F P+\mathrm{P} 19+A t \mathrm{Rec}_{\mathrm{TP}}- \\
\text { mCherry }\end{array}$ \\
\hline$O s \mathrm{Rbs} 1_{\mathrm{TP}}-e G F P+A t \operatorname{Rec} \mathrm{A}_{\mathrm{TP}}-\mathrm{mCherry}$ & pAGM4673 & $\begin{array}{c}O s \mathrm{Rbs} 1_{\mathrm{TP}}-e G F P+\mathrm{P} 19+A t \mathrm{Rec} \mathrm{A}_{\mathrm{TP}}- \\
\text { mCherry }\end{array}$ \\
\hline AtmRCA $A_{\mathrm{TP}}-n i f H_{\mathrm{nco}}-$ cytoe$G F P$ & pAGM4673 & $A t \mathrm{mRCA} \mathrm{TP}_{\mathrm{TP}}-n i f H_{\mathrm{nco}}+$ cytoe$G F P$ \\
\hline AtmBCCP1 $1_{\mathrm{TP}}-$ nif $M_{\mathrm{nco}}-$ cytoe $G F P$ & pAGM4673 & $A t \mathrm{mBCCP} 1_{\mathrm{TP}}-n i f M_{\mathrm{nco}}+$ cytoe$G F P$ \\
\hline$A t \mathrm{mTOCC} \mathrm{CP}_{\mathrm{TP}}-n i f U_{\mathrm{nco}}-$ cytoe $G F P$ & pAGM8055 & $A t \mathrm{mTOCC}$ TP-nif $U_{\text {nco }}+$ cytoe$G F P$ \\
\hline AtmCAB6 $6_{\mathrm{TP}}-n i f S_{\mathrm{nco}}$-cytoe$G F P$ & pAGM8055 & AtmCAB6 $6_{\mathrm{TP}}-n i f S_{\mathrm{nco}}+$ cytoe$G F P$ \\
\hline$A t \mathrm{mRCA} \mathrm{TP}_{\mathrm{TP}}-n i f H_{\mathrm{yco}}$-cytoe$G F P$ & pAGM4673 & $A t \mathrm{mRCA}_{\mathrm{TP}}-n i f H_{\mathrm{yco}}+$ cytoe$G F P$ \\
\hline AtmBCCP1 $1_{\mathrm{TP}}-$ nif $M_{\mathrm{yco}}-$ cytoe $G F P$ & pAGM4673 & AtmBCCP1 $1_{\mathrm{TP}}-n i f M_{\mathrm{yco}}-$ cytoe$G F P$ \\
\hline$A t \mathrm{mTOCC} \mathrm{CP}_{\mathrm{TP}}-n i f U_{\mathrm{yco}}-$ cytoe$G F P$ & pAGM8055 & $A t \mathrm{mTOCC}$ Tр-nif $U_{\text {yco }}+$ cytoe$G F P$ \\
\hline$A t \mathrm{mCAB} 6_{\mathrm{TP}}-n i f S_{\mathrm{yco}}-$ cytoe$G F P$ & pAGM8055 & AtmCAB6 $6_{\mathrm{TP}}-n i f S_{\mathrm{yco}}+$ cytoe $G F P$ \\
\hline AtmRCA ${ }_{\mathrm{TP}}-n i f H_{\mathrm{tsd}}-\mathrm{cyto} e G F P$ & pAGM4673 & $A t \mathrm{mRCA} \mathrm{TP}_{\mathrm{TP}} n i f H_{\mathrm{tsd}}+$ cytoe$G F P$ \\
\hline$A t \mathrm{mBCCP} 1_{\mathrm{TP}}-n i f M_{\mathrm{tsd}}-$ cytoe$G F P$ & pAGM4673 & AtmBCCP1 $1_{\mathrm{TP}}-n i f M_{\mathrm{tsd}}+$ cytoe$G F P$ \\
\hline AtmTOCC ${ }_{\mathrm{TP}}-n i f U_{\text {tsd }}$-cytoe$G F P$ & pAGM8055 & $A t \mathrm{mTOCC} \mathrm{TP}_{\mathrm{TP}}-n i f U_{\mathrm{tsd}}$-cytoe$G F P$ \\
\hline AtmCAB6 ${ }_{\mathrm{TP}}-n i f S_{\mathrm{tsd}}$-cytoe $G F P$ & pAGM8055 & AtmCAB6 $6_{\mathrm{TP}}-n i f S_{\mathrm{tsd}}+$ cytoe$G F P$ \\
\hline
\end{tabular}




\begin{tabular}{|c|c|c|}
\hline AtmRCA ${ }_{\text {TP-}} n i f H_{\text {rco-cytoe } G F P}$ & pAGM4673 & $A t \mathrm{mRCA}$ TP-nifH $H_{\text {rco }}+$ cytoe$G F P$ \\
\hline AtmBCCP1 $1_{\mathrm{TP}}-n i f M_{\text {rco-cytoe}} G F P$ & pAGM4673 & AtmBCCP1 $1_{\mathrm{TP}}-n i f M_{\mathrm{rco}}+$ cytoe $G F P$ \\
\hline AtmTOCC $\mathrm{TP}_{\mathrm{TP}}-$ nif $U_{\mathrm{rco}}$-cytoe $G F P$ & pAGM8055 & AtmTOCC $\mathrm{TP}_{\mathrm{TP}}-n i f U_{\text {rco }}+$ cytoe$G F P$ \\
\hline AtmCAB6 $_{\mathrm{TP}}-n i f S_{\mathrm{rco}}-$ cytoe $G F P$ & pAGM8055 & AtmCAB6 ${ }_{\mathrm{TP}}-n i f S_{\mathrm{rco}}+$ cytoe $G F P$ \\
\hline AtmRCA ${ }_{\mathrm{TP}}-n i f H_{\mathrm{rsd}}-$ cytoe$G F P$ & pAGM4673 & $A t \mathrm{mRCA} \mathrm{TP}_{\mathrm{TP}} n i f H_{\mathrm{rsd}}+$ cytoe$G F P$ \\
\hline AtmBCCP1 $1_{\mathrm{TP}}-n i f M_{\mathrm{rdd}}-$ cytoe $G F P$ & pAGM4673 & AtmBCCP1 $1_{\mathrm{TP}}-n i f M_{\mathrm{rd}}+$ cytoe $G F P$ \\
\hline AtmTOCC $\mathrm{TP}_{\mathrm{TP}}-n i f U_{\mathrm{rsd}}$-cytoe $G F P$ & pAGM8055 & $A t \mathrm{mTOCC} \mathrm{TP}_{\mathrm{TP}}-n i f U_{\mathrm{rsd}}+$ cytoe $G F P$ \\
\hline AtmCAB6 ${ }_{\mathrm{TP}}-n i f S_{\mathrm{rsd}}$-cytoe $G F P$ & pAGM8055 & AtmCAB6 $6_{\mathrm{TP}}-n i f S_{\mathrm{rsd}}+$ cytoe $G F P$ \\
\hline $\begin{array}{l}\text { mChloro-nifH } H_{r c o}(t G F P)- \\
n_{\text {nif }} M_{r c o}(m \text { Cherry })\end{array}$ & pAGM4673 & $\begin{array}{c}\text { AtmRCA } \mathrm{TP}_{\mathrm{TP}} \text { nif } H_{\mathrm{rco}-}-t G F P+\text { AtmBCCP1 } 1_{\mathrm{TP}-} \\
n i f M_{\mathrm{rco}}-m \text { Cherry }\end{array}$ \\
\hline mChloro $_{\text {rco-nifH-nifM-nifU-nifS (v1) }}$ & pUC57-L2 & $\begin{array}{c}A t \mathrm{mRCA} \\
A t \mathrm{TP}-n i f H_{\mathrm{rco}}+A t \mathrm{mBCCP} 1_{\mathrm{TP}}-n i f M_{\mathrm{rco}}+ \\
A \mathrm{CCC}_{\mathrm{TP}}-n i f U_{\mathrm{rco}}+A t \mathrm{mCAB} 6_{\mathrm{TP}}-n i f S_{\mathrm{rco}}\end{array}$ \\
\hline mChloro $_{\text {rco-nifH-nifM-nifU-nifS (v2) }}$ & pUC57-L2 & $\begin{array}{c}A t \mathrm{mRCA}_{\mathrm{TP}}-n i f H_{\mathrm{rco}}+A t \mathrm{mBCCP} 1_{\mathrm{TP}}-n i f M_{\mathrm{rco}} \\
(\mathrm{b})+A t \mathrm{mTOCC} \mathrm{TP}_{\mathrm{TP}}-n i f U_{\mathrm{rco}}(\mathrm{b})+A t \mathrm{mCAB} 6_{\mathrm{TP}}- \\
n i f S_{\mathrm{rco}}(\mathrm{b})\end{array}$ \\
\hline 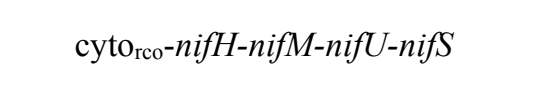 & pUC57-L2 & $\begin{array}{c}\text { cytonif }_{\text {rco }}+ \\
\text { cytonif } M_{\text {rco }}+\text { cytonif } U_{\text {rco }}+ \\
\text { cytonif } S_{\text {rco }}\end{array}$ \\
\hline
\end{tabular}

Abbreviation: m: minimal; c: complete; yco: Yeast codon optimization; tsd: Tobacco synthetic design; rco: Rice codon optimization; rsd: Rice synthetic design. 
Supplemental Table 5. Optimization and motif settings used in Codon Optimization On-Line for tobacco nif synthetic designed genes.

\begin{tabular}{ll}
\hline Optimization settings & \\
\hline Parameter & Setting \\
\hline Individual Codon Usage & Maximize \\
Codon Context & Ignore \\
Codon Adaptation Index & Ignore \\
Number of Hidden Stop Codons & Ignore \\
5' RNA Folding Instability & Maximize in the first $50 \mathrm{bp}$ \\
GC Total Content & $43.38 \%$ \\
GC3 Content & $39.53 \%$ \\
Custom Codon Usage Pattern Values & Imported from $\underline{\text { http://www.kazusa.or.jp/codon/cgi- }}$ \\
& $\underline{\text { bin/showcodon.cgi?species=4097 }}$
\end{tabular}

\begin{tabular}{|c|c|c|c|c|}
\hline \multicolumn{5}{|c|}{ Motif settings } \\
\hline Parameter & Setting & & & \\
\hline \multirow{28}{*}{$\begin{array}{l}\text { Exclusion } \\
\text { sequences }\end{array}$} & Constraint & Description & Sequence & Reference \\
\hline & & PolyA.1 & AATGAA & \\
\hline & & PolyA.2 & AATATT & \\
\hline & & PolyA.3 & GATAAA & 4 \\
\hline & & PolyA.4 & AATTAA & \\
\hline & & PolyA. 5 & AATAAT & \\
\hline & & FUE1 & AATGTA & \\
\hline & & FUE2 & TAGTAG & \\
\hline & & FUE3 & TGATTC & \\
\hline & & FUE4 & CAAGTT & 5 \\
\hline & & FUE5 & TAATAA & \\
\hline & & FUE6 & TTCTTC & \\
\hline & & NUE1 & AATAAA & \\
\hline & & Cryptic splice donor & MAGGTRAGT & \\
\hline & & $\begin{array}{l}\text { Cryptic splice } \\
\text { acceptor }\end{array}$ & YYYYNTAGG & \\
\hline & & $\begin{array}{l}\text { RNA destabilizing } \\
\text { sequence }\end{array}$ & ATTTA & \\
\hline & & $\begin{array}{l}\text { RNA instability } \\
\text { determinant }\end{array}$ & ATAGAT & \\
\hline & & Shine Dalgarno & AGGAGGTNNNNNDTG & \\
\hline & & ATRICH1 & ASAAAA & \\
\hline & & ATRICH2 & AASAAA & 6 \\
\hline & & ATRICH3 & AAASAA & \\
\hline & & ATRICH4 & AAAASA & \\
\hline & & ATRICH5 & TSAAAA & \\
\hline & & ATRICH6 & TTSTTT & \\
\hline & & ATRICH7 & TTTSTT & \\
\hline & & ATRICH8 & TTTTST & \\
\hline & & NUE2 & AAAAAA & \\
\hline & & NUE2 & TTTTTT & \\
\hline
\end{tabular}




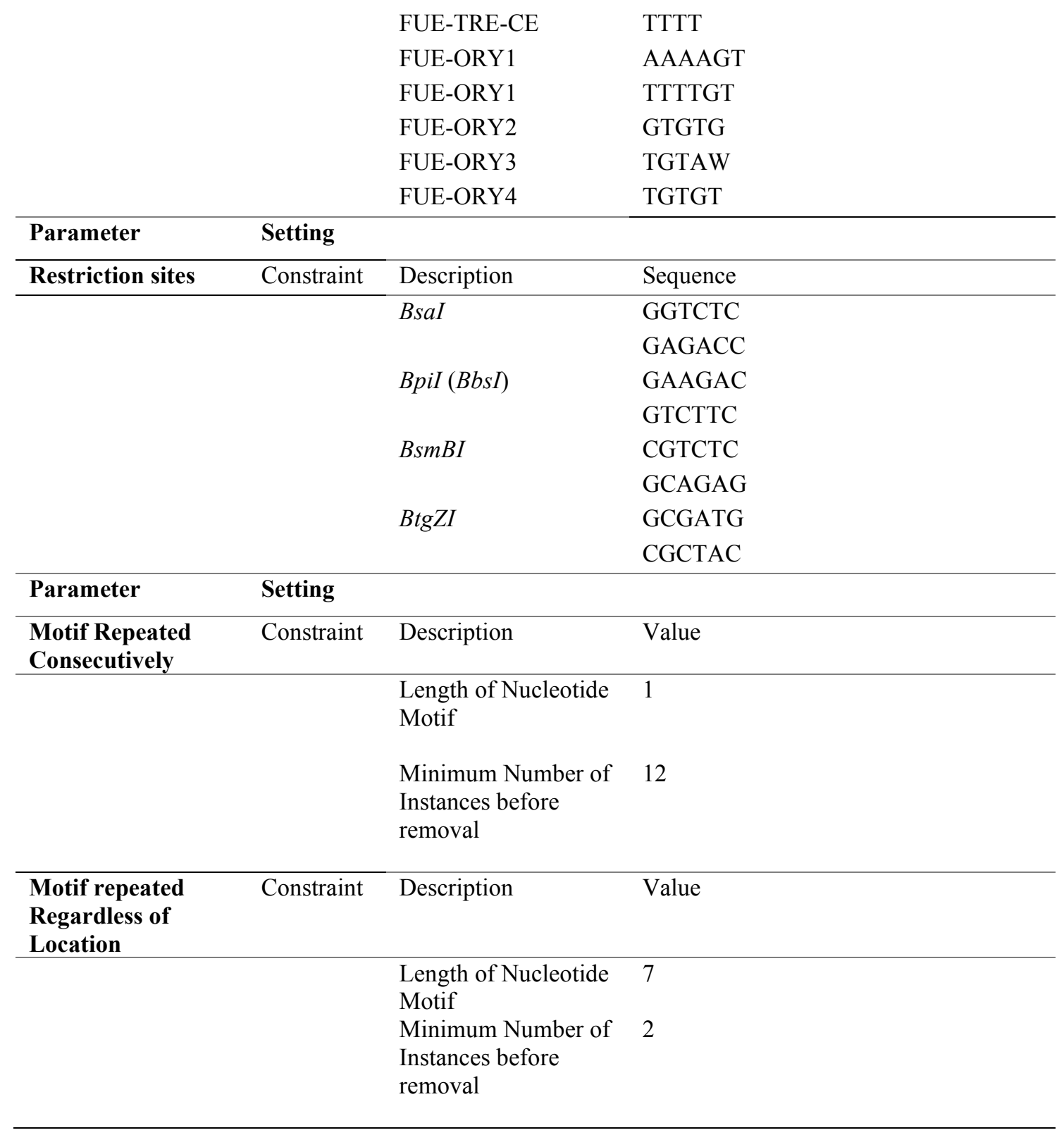

Abbreviation: ICU: Individual Codon Usage; GC: Distribution of Guanine and Cytosine (GC) content in ORFs; GC3: Distribution of Guanine and Cytosine (GC) content in the third codon position; FUE: Far Upstream Element; NUE: Near Upstream Element. 
Supplemental Table 6. Characteristics of yeast codon-optimized and tobacco synthetic designed sequences.

\begin{tabular}{|c|c|c|c|c|c|c|c|c|}
\hline & \multicolumn{2}{|c|}{ AvnifH } & \multicolumn{2}{|c|}{ AvnifM } & \multicolumn{2}{|c|}{ AvnifU } & \multicolumn{2}{|c|}{ AvnifS } \\
\hline & yco & tsd & yco & tsd & yco & tsd & yco & tsd \\
\hline CAI & 0.82 & 0.76 & 0.84 & 0.77 & 0.81 & 0.79 & 0.82 & 0.72 \\
\hline ICU Fitness & -0.19641 & -0.086724 & -0.1717 & -0.08324 & -0.17922 & -0.088254 & -0.1632 & -0.07813 \\
\hline $\begin{array}{c}\text { Total GC } \\
\text { Content }\end{array}$ & $40.09 \%$ & $43.87 \%$ & $40.7 \%$ & $43.76 \%$ & $43.34 \%$ & $43.77 \%$ & $41.11 \%$ & $45.24 \%$ \\
\hline GC3 Content & $33.68 \%$ & $39.52 \%$ & $28.57 \%$ & $35.71 \%$ & $34.19 \%$ & $34.5 \%$ & $30.77 \%$ & $35.98 \%$ \\
\hline $\begin{array}{l}\text { Exclusion } \\
\text { Motifs (bp) }\end{array}$ & 81 & 5 & 115 & 0 & 88 & 0 & 149 & 6 \\
\hline $\begin{array}{l}\text { Repetitions } \\
\text { (bp) }\end{array}$ & 463 & 14 & 461 & 0 & 454 & 0 & 644 & 0 \\
\hline
\end{tabular}

Abbreviation: yco: Yeast codon optimization; tsd: Tobacco synthetic design; CAI: Codon Adaptation Index; ICU Fitness: how far the sequence deviates from the target Individual Codon Usage; GC: Distribution of Guanine and Cytosine (GC) content in ORFs; GC3: Distribution of Guanine and Cytosine (GC) content in the third codon position. 
Supplemental Table 7. Summary of Chloroplast Transit Peptides studied in this thesis.

\begin{tabular}{|c|c|c|c|c|c|c|}
\hline TP name & & Length & Sequence & $\begin{array}{l}\sim \mathrm{Mw} \\
(\mathrm{kDa})\end{array}$ & $\begin{array}{c}\text { Related } \\
\text { protein } \\
\text { (UniProt } \\
\text { ID) } \\
\end{array}$ & Reference \\
\hline \multirow[b]{2}{*}{$\operatorname{AtAROA}_{\mathrm{TP}}$} & $\mathrm{m}$ & $1-42$ & $\begin{array}{l}\text { MAQVSRICNGVQNPSLISNLSKSSQRKSPLSVSLKTQ } \\
\text { QHPRA }\end{array}$ & 4.57 & \multirow{2}{*}{ P05466 } & \multirow{2}{*}{7} \\
\hline & $\mathrm{c}$ & $1-76$ & $\begin{array}{l}\text { MAQVSRICNGVQNPSLISNLSKSSQRKSPLSVSLKTQ } \\
\text { QHPRAYPISSSWGLKKSGMTLIGSELRPLKVMSSVST } \\
\text { AE }\end{array}$ & 8.20 & & \\
\hline \multirow{2}{*}{$A t \mathrm{BCCP} 1_{\mathrm{TP}}$} & $\mathrm{m}$ & $1-62$ & $\begin{array}{l}\text { MASSSFSVTSPAAAASVYAVTQTSSHFPIQNRSRRVS } \\
\text { FRLSAKPKLRELSKPSRSSYPVVKA }\end{array}$ & 6.73 & \multirow{2}{*}{ Q42533 } & \multirow{2}{*}{8} \\
\hline & $\mathrm{c}$ & $1-80$ & $\begin{array}{l}\text { MASSSFSVTSPAAAASVYAVTQTSSHFPIQNRSRRVS } \\
\text { FRLSAKPKLRFLSKPSRSSYPVVKAQSNKVSTGASSN } \\
\text { AAKVDG }\end{array}$ & 8.43 & & \\
\hline \multirow{2}{*}{$A t \mathrm{CAB} 6_{\mathrm{TP}}$} & $\mathrm{m}$ & $1-56$ & $\begin{array}{l}\text { MASNSLMSCGIAAVYPSLLSSSKSKFVSAGVPLPNAG } \\
\text { NVGRIRMAAHWMPGEPRPA }\end{array}$ & 5.77 & \multirow{2}{*}{ Q01667 } & \multirow{2}{*}{8,9} \\
\hline & $\mathrm{c}$ & $1-67$ & $\begin{array}{l}\text { MASNSLMSCGIAAVYPSLLSSSKSKFVSAGVPLPNAG } \\
\text { NVGRIRMAAHWMPGEPRPAYLDGSAPGDFG }\end{array}$ & 6.85 & & \\
\hline \multirow{2}{*}{$A t \mathrm{DNAJ} 8_{\mathrm{TP}}$} & $\mathrm{m}$ & $1-47$ & $\begin{array}{l}\text { MTIALTIGGNGFSGLPGSSFSSSSSSFRLKNSRRKNT } \\
\text { KMLNRSKVVC }\end{array}$ & 5.01 & \multirow{2}{*}{ Q9SAG8 } & \multirow{2}{*}{8} \\
\hline & $\mathrm{c}$ & $1-80$ & $\begin{array}{l}\text { MTIALTIGGNGFSGLPGSSFSSSSSSFRLKNSRRKNT } \\
\text { KMLNRSKVVCSSSSSVMDPYKTLKIRPDSSEYEVKKA } \\
\text { FRQLAK }\end{array}$ & 8.77 & & \\
\hline \multirow{2}{*}{$A t \mathrm{GLTB} 2_{\mathrm{TP}}$} & $\mathrm{m}$ & $1-73$ & $\begin{array}{l}\text { MALQSPGATGASSSVSRLLSSAKLSSTKTIFSVDFVR } \\
\text { SYCISKGTKRRNELSGFRGYSPLLKSSLRSPFSVKA }\end{array}$ & 7.79 & \multirow{2}{*}{ Q9T0P4 } & \multirow{2}{*}{8} \\
\hline & c & $1-80$ & $\begin{array}{l}\text { MALQSPGATGASSSVSRLLSSAKLSSTKTIFSVDFVR } \\
\text { SYCISKGTKRRNELSGFRGYSPLLKSSLRSPFSVKAI } \\
\text { LNSDRA }\end{array}$ & 8.56 & & \\
\hline \multirow{2}{*}{$N t \mathrm{RBS}_{\mathrm{TP}}$} & $\mathrm{m}$ & $1-20$ & MASSVLSSAAVATRSNVAQA & 1.92 & \multirow{2}{*}{ P69249 } & \multirow{2}{*}{10} \\
\hline & $\mathrm{c}$ & $1-57$ & $\begin{array}{l}\text { MASSVLSSAAVATRSNVAQANMVAPFTGLKSAASFPV } \\
\text { SRKQNLDITSIASNGGRVQC }\end{array}$ & 5.77 & & \\
\hline \multirow{2}{*}{$\operatorname{AtRBS1\mathrm {A}_{\mathrm {TP}}}$} & $\mathrm{m}$ & $1-55$ & $\begin{array}{l}\text { MASSMLSSATMVASPAQATMVAPFNGLKSSAAFPATR } \\
\text { KANNDITS ITSNGGRVNC }\end{array}$ & 5.53 & \multirow{2}{*}{ P10795 } & \multirow{2}{*}{9} \\
\hline & $\mathrm{c}$ & $1-79$ & $\begin{array}{l}\text { MASSMLSSATMVASPAQATMVAPFNGLKSSAAFPATR } \\
\text { KANNDITSITSNGGRVNCMQVWPPIGKKKFETLSYLP } \\
\text { DLTDS }\end{array}$ & 8.31 & & \\
\hline \multirow{2}{*}{$P s \mathrm{RBS} 2 \mathrm{TP}$} & $\mathrm{m}$ & $1-22$ & MASMISSSAVTTVSRASRGQSA & 2.18 & \multirow{2}{*}{ P00869 } & \multirow{2}{*}{11} \\
\hline & c & $1-57$ & $\begin{array}{l}\text { MASMISSSAVTTVSRASRGQSAAVAPFGGLKSMTGFP } \\
\text { VKKVNTDITSITSNGGRVKC }\end{array}$ & 5.75 & & \\
\hline
\end{tabular}




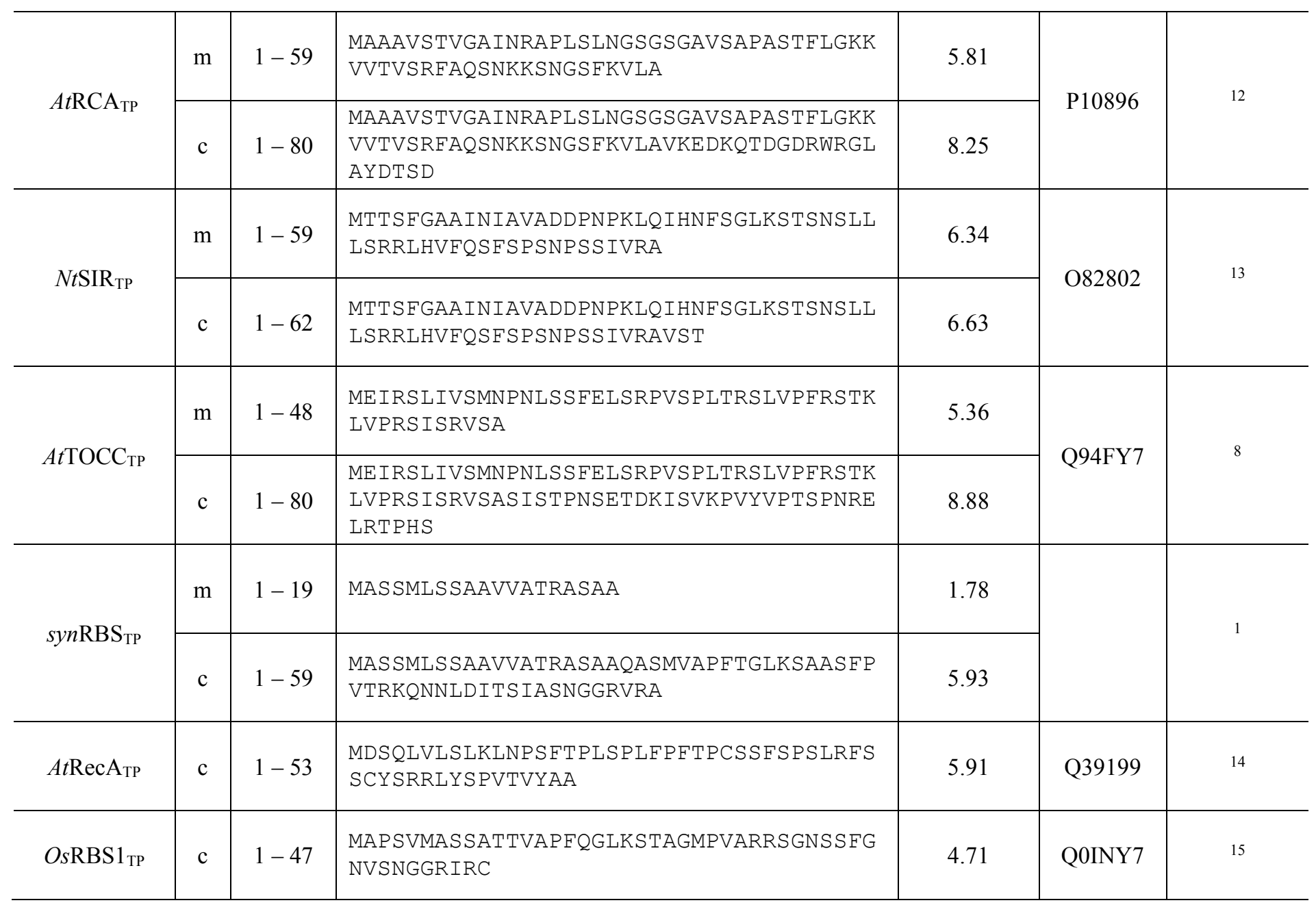

Abbreviation: m: minimal; c: complete. 
Supplemental Table 8. Summary of processing products from TP-Nif fusions.

Western blot signals of the product of expressing 12 TPs in full version (complete) and up until their predicted cleavage site by stromal peptidase (minimal) fused to the N-terminus of NifH, NifM, NifU and NifS, on a scale indicating: -: Nif proteins with the same electrophoretic mobility as their cytosolic controls; + : Nif proteins with lower electrophoretic mobility as their cytosolic controls; and $=$ : Nif proteins with incomplete processing.

\begin{tabular}{|c|c|c|c|c|}
\hline & NifH & NifM & NifU & NifS \\
\hline $\operatorname{AtmAROA}_{\mathrm{TP}}$ & - & $=$ & + & $=$ \\
\hline $\operatorname{AtcAROA}_{\mathrm{TP}}$ & + & - & - & - \\
\hline AtmCAB6 $6_{\mathrm{TP}}$ & + & $=$ & - & - \\
\hline$A t \mathrm{cCAB} 6_{\mathrm{TP}}$ & + & + & + & $=$ \\
\hline AtmDNAJ8 $8_{\mathrm{TP}}$ & + & $=$ & - & - \\
\hline AtcDNAJ8 $8_{\mathrm{TP}}$ & + & $=$ & + & $=$ \\
\hline AtmGLTB2 ${ }_{\mathrm{TP}}$ & - & - & - & - \\
\hline AtcGLTB2 $2_{\mathrm{TP}}$ & + & + & + & + \\
\hline$A t \mathrm{mTOCC} \mathrm{CP}_{\mathrm{TP}}$ & - & $=$ & - & + \\
\hline$A t \mathrm{cTOCC} \mathrm{TP}_{\mathrm{T}}$ & + & + & + & + \\
\hline$N t m R B S_{\text {TP }}$ & - & + & + & + \\
\hline$N t c \mathrm{RBS}_{\mathrm{TP}}$ & - & $=$ & - & - \\
\hline$P s \mathrm{mRBS} 2_{\mathrm{TP}}$ & $=$ & + & + & + \\
\hline$P s c R B S 2_{\mathrm{TP}}$ & + & $=$ & - & - \\
\hline$A t \mathrm{mBCCP} 1_{\mathrm{TP}}$ & - & - & - & - \\
\hline$A t \mathrm{cBCCP} 1_{\mathrm{TP}}$ & + & + & + & + \\
\hline$N t \mathrm{mSIR} \mathrm{R}_{\mathrm{TP}}$ & - & $=$ & - & - \\
\hline$N t c \mathrm{SIR}_{\mathrm{TP}}$ & - & $=$ & - & + \\
\hline AtmRBS1 $\mathrm{A}_{\mathrm{TP}}$ & - & $=$ & $=$ & - \\
\hline$A t c R B S 1 \mathrm{~A}_{\mathrm{TP}}$ & + & $=$ & + & + \\
\hline$A t \mathrm{mRCA} \mathrm{AP}_{\mathrm{TP}}$ & - & + & - & - \\
\hline$A t c \mathrm{RCA}_{\mathrm{TP}}$ & + & + & + & + \\
\hline$s y n \mathrm{mRBS}_{\mathrm{TP}}$ & - & + & + & + \\
\hline$s y n \mathrm{RBS}_{\mathrm{TP}}$ & - & $=$ & - & - \\
\hline
\end{tabular}


Supplemental Table 9. Motif settings used for rice nif synthetic designed genes.

\begin{tabular}{|c|c|c|c|c|}
\hline \multicolumn{5}{|c|}{ Motif settings } \\
\hline Parameter & Setting & & & \\
\hline \multirow{38}{*}{$\begin{array}{l}\text { Exclusion } \\
\text { sequences }\end{array}$} & Constraint & Description & Sequence & Reference \\
\hline & & PolyA.1 & AATGAA & \\
\hline & & PolyA.2 & AATATT & \\
\hline & & PolyA.3 & GATAAA & 4 \\
\hline & & PolyA.4 & AATTAA & \\
\hline & & PolyA.5 & AATAAT & \\
\hline & & FUE1 & TTAATT & \\
\hline & & FUE2 & TGTAAA & \\
\hline & & FUE3 & AATAAA & \\
\hline & & FUE4 & TTCAGA & \\
\hline & & FUE5 & TAGTAG & \\
\hline & & FUE6 & TTCTTT & 5 \\
\hline & & FUE7 & GCGGCG & \\
\hline & & NUE1 & AATAAA & \\
\hline & & NUE2 & TTAATT & \\
\hline & & NUE3 & TAGTAG & \\
\hline & & NUE4 & GATCGA & \\
\hline & & CE1 & TAATTA & \\
\hline & & Cryptic splice donor & MAGGTRAGT & \\
\hline & & $\begin{array}{l}\text { Cryptic splice } \\
\text { acceptor }\end{array}$ & YYYYNTAGG & \\
\hline & & $\begin{array}{l}\text { RNA destabilizing } \\
\text { sequence }\end{array}$ & ATTTA & \\
\hline & & $\begin{array}{l}\text { RNA instability } \\
\text { determinant }\end{array}$ & ATAGAT & \\
\hline & & Shine Dalgarno & AGGAGGTNNNNNDTG & \\
\hline & & ATRICH1 & ASAAAA & \\
\hline & & ATRICH2 & AASAAA & \\
\hline & & ATRICH3 & AAASAA & \\
\hline & & ATRICH4 & AAAASA & 6 \\
\hline & & ATRICH5 & TSAAAA & \\
\hline & & ATRICH6 & TTSTTT & \\
\hline & & ATRICH7 & TTTSTT & \\
\hline & & ATRICH8 & TTTTST & \\
\hline & & NUE2 & AAAAAA & \\
\hline & & FUE-TRE-CE & TTTT & \\
\hline & & FUE-ORY1 & TTTTGT & \\
\hline & & FUE-ORY2 & GTGTG & \\
\hline & & FUE-ORY3 & TGTAW & \\
\hline & & FUE-ORY4 & TGTGT & \\
\hline & & FUE-ORY5 & WTGTA & \\
\hline
\end{tabular}




\begin{tabular}{llll}
\hline Parameter & Setting & & \\
\hline Restriction sites & Constraint & Description & Sequence \\
\hline & BsaI & GGTCTC \\
& & GAGACC \\
& BpiI $(B b s I)$ & GAAGAC \\
& & GTCTTC \\
& BsmBI & CGTCTC \\
& & GCAGAG \\
& BtgZI & GCGATG \\
& & CGCTAC
\end{tabular}

Abbreviation: FUE: Far Upstream Element; NUE: Near Upstream Element. 
Supplemental Table 10. Exclusion motifs present in codon-optimized nif genes.

Number of times the following exclusion motifs were found in rice codon-optimized nif genes provided by Genescript, which were subsequently removed by PCR.

\begin{tabular}{|c|c|c|c|c|c|}
\hline Name & Motif & nifH & nifM & nifU & nifs \\
\hline \multicolumn{6}{|c|}{ Removed by PCR } \\
\hline FUE7 & GCGGCG & 3 & 1 & 3 & \\
\hline FUE4 & TTCAGA & & 1 & & \\
\hline NUE4 & GATCGA & & & 3 & 1 \\
\hline \multicolumn{6}{|c|}{ Maintained } \\
\hline Shine D'algarno & GCAGAG & 4 & 4 & 5 & 5 \\
\hline FUE ORY2 & WTGTA & 2 & 1 & & \\
\hline FUE ORY5 & CGCTAC & 1 & & & \\
\hline$B s m B I$ & GCAGAG & 1 & 2 & & \\
\hline BtgZI & CGCTAC & & 1 & & \\
\hline
\end{tabular}


Supplemental Table 11. Characteristics of $n i f H$ and nif $M$ sequences regarding expression in rice.

The CAI index was obtained from the Biologics International Corp codon adaptation index calculator and rare codon analyser using Zea mays as target setting.

\begin{tabular}{|c|c|c|c|c|c|c|c|c|c|c|}
\hline & \multicolumn{5}{|c|}{ AvnifH } & \multicolumn{5}{|c|}{ AvnifM } \\
\hline & 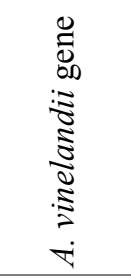 & 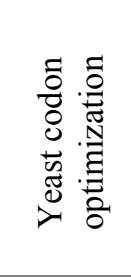 & 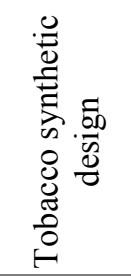 & 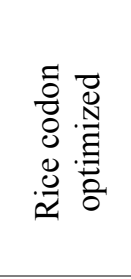 & 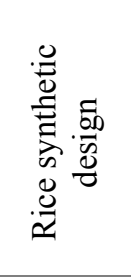 & 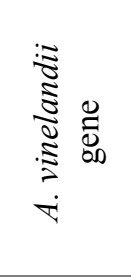 & 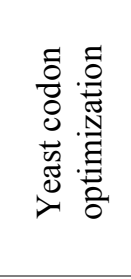 & 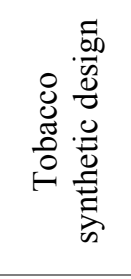 & 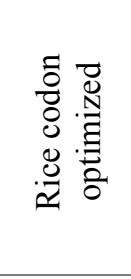 & 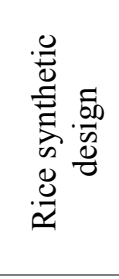 \\
\hline CAI & 0.83 & 0.57 & 0.60 & 0.90 & 0.89 & 0.85 & 0.53 & 0.56 & 0.89 & 0.89 \\
\hline $\begin{array}{c}\text { Total GC } \\
\text { Content }\end{array}$ & $58.42 \%$ & $39.86 \%$ & $43.87 \%$ & $61.03 \%$ & $60.69 \%$ & $65.42 \%$ & $40.73 \%$ & $43.76 \%$ & $64.73 \%$ & $64.73 \%$ \\
\hline $\begin{array}{c}\text { GC3 } \\
\text { Content }\end{array}$ & $76.98 \%$ & $33.33 \%$ & $39.52 \%$ & $85.86 \%$ & $84.83 \%$ & $84.01 \%$ & $28.67 \%$ & $35.71 \%$ & $83.96 \%$ & $83.96 \%$ \\
\hline $\begin{array}{c}\text { Exclusion } \\
\text { Motifs }\end{array}$ & 7 & 23 & 4 & 11 & 8 & 11 & 21 & 1 & 10 & 8 \\
\hline
\end{tabular}

Abbreviation: CAI: Codon Adaptation Index; GC: Distribution of Guanine and Cytosine (GC) content in ORFs; GC3: Distribution of Guanine and Cytosine (GC) content in the third codon position. 
Supplemental Table 12. Statistics of import efficiency of TPs.

(A) Import efficiency of TPs into Arabidopsis chloroplast and root plastids, and rice chloroplast in transient expression protoplast experiments. (B) Import efficiency of TPs into rice proplastids, chloroplast and root plastids. Import efficiency was defined as the percentage of the processed faster-migrating protein form relative to the total amount of expressed eGFP protein. Data represent means $(n=3)$ with $\mathrm{SD}$, except GLTB2 and RBS1 TPs tested in root plastids, where $\mathrm{n}=2$. (C-E) Multiple Student's test between tissues in protoplast transient experiments at (C) 12 hours and (E) $16 \mathrm{~h}$ post-transfection and (D) stable transgenic rice lines. Student's test significant differences $(P<0.05)$.

(A)

Arabidopsis chloroplast

Arabidopsis leucoplast

Rice chloroplast

\begin{tabular}{|c|c|c|c|c|c|c|c|c|c|}
\hline Protoplast & $12 \mathrm{~h}$ & $16 \mathrm{~h}$ & $\mathrm{~N}$ & $12 \mathrm{~h}$ & $16 \mathrm{~h}$ & $\mathrm{~N}$ & $12 \mathrm{~h}$ & $16 \mathrm{~h}$ & $\mathrm{~N}$ \\
\hline$A t \mathrm{mCAB} 6_{\mathrm{TP}}$ & $95,82 \pm 1,94$ & $95,20 \pm 0,96$ & 3 & $60,57 \pm 1,36$ & $71,24 \pm 9,51$ & 3 & $80,95 \pm 4,03$ & $81,06 \pm 2,98$ & 3 \\
\hline AtmRCA $\mathrm{TP}_{\mathrm{TP}}$ & $92,32 \pm 5,66$ & $94,32 \pm 4,88$ & 3 & $56,16 \pm 3,34$ & $53,28 \pm 0,75$ & 3 & $85,60 \pm 4,06$ & $88,50 \pm 2,16$ & 3 \\
\hline$A t \mathrm{mTOCC}$ & $71,41 \pm 15,94$ & $63,09 \pm 17,67$ & 3 & $50,80 \pm 3,53$ & $52,01 \pm 8,79$ & 3 & $89,87 \pm 0,44$ & $92,10 \pm 1,30$ & 3 \\
\hline$A t \mathrm{mBCCP} 1_{\mathrm{TP}}$ & $98,99 \pm 0,90$ & $99,57 \pm 0,19$ & 3 & $97,51 \pm 0,31$ & $96,70 \pm 3,39$ & 3 & $94,47 \pm 1,95$ & $98,42 \pm 1,40$ & 3 \\
\hline AtcGLBT2 2 TP & $56,87 \pm 2,66$ & $79,46 \pm 7,93$ & 3 & $50,78 \pm 1,99$ & $49,18 \pm 3,95$ & 2 & $97,64 \pm 1,92$ & $99,07 \pm 0,68$ & 3 \\
\hline$O s \mathrm{RBS} 1_{\mathrm{TP}}$ & $2,57 \pm 1,74$ & $2,39 \pm 0,76$ & 3 & $3,28 \pm 3,36$ & $2,29 \pm 1,88$ & 2 & $68,38 \pm 0,53$ & $68,24 \pm 4,47$ & 3 \\
\hline
\end{tabular}

(B)

\begin{tabular}{ccccccc}
\hline Stable rice lines & Callus proplastids & \multicolumn{2}{c}{ Leaf chloroplast } & \multicolumn{2}{c}{ Root leucoplast } \\
\hline & $\% \pm \mathrm{SD}$ & $\mathrm{N}$ & $\% \pm \mathrm{SD}$ & $\mathrm{N}$ & $\% \pm \mathrm{SD}$ & $\mathrm{N}$ \\
\hline AtmCAB6 & $99,41 \pm 0,78$ & 3 & $98,64 \pm 0,98$ & 3 & $99,93 \pm 0,07$ & 3 \\
AtmRCA $_{\mathrm{TP}}$ & $99,55 \pm 0,16$ & 3 & $98,03 \pm 1,46$ & 3 & $99,46 \pm 0,09$ & 3 \\
AtmTOCC $_{\mathrm{TP}}$ & $97,83 \pm 1,39$ & 3 & $99,66 \pm 0,28$ & 3 & $99,68 \pm 0,19$ & 3 \\
AtmBCCP1 $_{\mathrm{TP}}$ & $99,75 \pm 0,38$ & 3 & - & - & - & - \\
\hline
\end{tabular}


(C)

\begin{tabular}{cccc|ccc}
\hline 12 hours & $\begin{array}{c}\text { Arabidopsis } \\
\text { chloroplast }\end{array}$ & $\begin{array}{c}\text { Arabidopsis } \\
\text { leucoplast }\end{array}$ & p-value & $\begin{array}{c}\text { Arabidopsis } \\
\text { chloroplast }\end{array}$ & $\begin{array}{c}\text { Rice } \\
\text { chloroplast }\end{array}$ & p-value \\
\hline AtmCAB6 & 95,82 & 60,57 & 0,000014 & 95,82 & 80,95 & 0,004532 \\
AtmRCA & 92,32 & 56,16 & 0,000676 & 92,32 & 85,60 & 0,169540 \\
AtmTOCC & 71,41 & 50,80 & 0,094104 & 71,41 & 89,87 & 0,115386 \\
AtmBCCP1 & 98,99 & 97,51 & 0.055017 & 98,99 & 94,47 & 0.021856 \\
AtcGLBT2 & 56,87 & 50,78 & 0,072910 & 56,87 & 97,64 & 0,000027 \\
OsRBS1 & 2,568 & 3,280 & 0,766732 & 2,568 & 68,38 & $<0,00000$ \\
\hline
\end{tabular}

(D)

\begin{tabular}{cccc|ccc}
\hline 16 hours & $\begin{array}{c}\text { Arabidopsis } \\
\text { chloroplast }\end{array}$ & $\begin{array}{c}\text { Arabidopsis } \\
\text { leucoplast }\end{array}$ & p-value & $\begin{array}{c}\text { Arabidopsis } \\
\text { chloroplast }\end{array}$ & $\begin{array}{c}\text { Rice } \\
\text { chloroplast }\end{array}$ & p-value \\
\hline AtmCAB6 & 95,20 & 71,24 & 0,012263 & 95,20 & 81,06 & 0,001446 \\
$A t m R C A_{\mathrm{TP}}$ & 94,32 & 53,28 & 0,000135 & 94,32 & 88,50 & 0,131998 \\
$A t m$ mOCC & 63,09 & 52,01 & 0,386146 & 63,09 & 92,10 & 0,047101 \\
$A t m B C C P 1_{\mathrm{TP}}$ & 99,57 & 96,70 & 0.217164 & 99,57 & 98,42 & 0.232171 \\
AtcGLBT2 & 79,46 & 49,18 & 0,016899 & 79,46 & 99,07 & 0,012967 \\
OsRBS1 & 2,390 & 2,288 & 0,934972 & 2,390 & 68,24 & 0,000015 \\
\hline
\end{tabular}

(E)

\begin{tabular}{|c|c|c|c|c|c|c|c|c|c|}
\hline $\begin{array}{c}\text { Stable rice } \\
\text { lines }\end{array}$ & $\begin{array}{c}\text { Callus } \\
\text { proplastids } \\
\end{array}$ & $\begin{array}{c}\text { Leaf } \\
\text { chloroplast } \\
\end{array}$ & p-value & $\begin{array}{c}\text { Callus } \\
\text { proplastids } \\
\end{array}$ & $\begin{array}{c}\text { Root } \\
\text { leucoplast }\end{array}$ & p-value & $\begin{array}{c}\text { Leaf } \\
\text { chloroplast }\end{array}$ & $\begin{array}{c}\text { Root } \\
\text { leucoplast }\end{array}$ & p-value \\
\hline AtmCAB6 & 99,41 & 98,64 & 0,345617 & 99,41 & 99,93 & 0,313229 & 98,64 & 99,93 & 0,085177 \\
\hline $\operatorname{AtmRCA}_{\mathrm{TP}}$ & 99,55 & 98,03 & 0,147788 & 99,55 & 99,46 & 0,443386 & 98,03 & 99,46 & 0,165593 \\
\hline$A t \mathrm{mTOCC}$ & 97,83 & 99,66 & 0,088105 & 97,83 & 99,68 & 0,084426 & 99,66 & 99,68 & 0,949813 \\
\hline AtmBCCP1 $1_{\mathrm{TP}}$ & - & - & - & - & - & - & - & - & - \\
\hline
\end{tabular}


Supplemental Table 13. Levels of immunogold labelling.

Number of gold particles observed on organelles and cytoplasm of cells of different samples and lines after using a monoclonal or polyclonal antiserum, on a scale indicating: - (not labelling), +/(very light labelling), + (weak but specific labelling), ++ (specific labelling), +++ (more specific labelling) and ++++ (specific and strong labelling).

\begin{tabular}{|c|c|c|c|c|c|c|}
\hline $\begin{array}{l}\text { Antiserum: } \\
\text { Monoclonal }\end{array}$ & & WT & $\begin{array}{l}A t m C A B 6 \\
\text { eGFP }\end{array}$ & $\begin{array}{c}\operatorname{AtmRCA}_{\mathrm{TP}} \\
\text {-eGFP }\end{array}$ & $\begin{array}{c}A t \mathrm{ATOCC} \\
\text { eGFP }\end{array}$ & $\begin{array}{c}\operatorname{AtmBCCP} 1_{\mathrm{TP}-} \\
\text { eGFP }\end{array}$ \\
\hline \multirow{4}{*}{ Callus } & Plastids & - & + & ++ & + & + \\
\hline & Mitochondria & - & - & - & - & - \\
\hline & $\begin{array}{l}\text { Nuclei/nucle } \\
\text { oli }\end{array}$ & - & - & - & - & $+/-$ \\
\hline & Cytoplasm & - & - & - & - & - \\
\hline \multirow{4}{*}{ Leaf } & Chloroplasts & - & + & +++ & + & $\mathrm{n} / \mathrm{a}$ \\
\hline & Mitochondria & - & - & - & - & $\mathrm{n} / \mathrm{a}$ \\
\hline & $\begin{array}{l}\text { Nuclei/nucle } \\
\text { oli }\end{array}$ & - & - & - & - & $\mathrm{n} / \mathrm{a}$ \\
\hline & Cytoplasm & - & - & - & - & $\mathrm{n} / \mathrm{a}$ \\
\hline \multirow{4}{*}{ Root } & Plastids & - & + & ++ & + & $\mathrm{n} / \mathrm{a}$ \\
\hline & Mitochondria & - & - & - & - & $\mathrm{n} / \mathrm{a}$ \\
\hline & $\begin{array}{l}\text { Nuclei/nucle } \\
\text { oli }\end{array}$ & - & - & - & - & $\mathrm{n} / \mathrm{a}$ \\
\hline & Cytoplasm & - & - & - & - & $\mathrm{n} / \mathrm{a}$ \\
\hline $\begin{array}{l}\text { Antiserum: } \\
\text { Polyclonal }\end{array}$ & & WT & $\begin{array}{l}A t m C A B 6 T \text { - } \\
\text { eGFP }\end{array}$ & $\begin{array}{c}\text { AtmRCATP } \\
\text {-eGFP }\end{array}$ & $\begin{array}{c}A t \mathrm{tmTOCC} \text { тр- } \\
\text { eGFP }\end{array}$ & $\begin{array}{c}\operatorname{AtmBCCP1} 1 \text { TP- } \\
\text { eGFP }\end{array}$ \\
\hline \multirow{4}{*}{ Callus } & Plastids & - & +++ & ++++ & ++ & $\mathrm{n} / \mathrm{a}$ \\
\hline & Mitochondria & - & - & - & - & $\mathrm{n} / \mathrm{a}$ \\
\hline & $\begin{array}{l}\text { Nuclei/nucle } \\
\text { oli }\end{array}$ & + & + & + & + & $\mathrm{n} / \mathrm{a}$ \\
\hline & Cytoplasm & $+/-$ & $+/-$ & $+/-$ & $+/-$ & $\mathrm{n} / \mathrm{a}$ \\
\hline \multirow{4}{*}{ Leaf } & Chloroplasts & $+/-$ & +++ & ++++ & ++ & $\mathrm{n} / \mathrm{a}$ \\
\hline & Mitochondria & - & - & - & - & $\mathrm{n} / \mathrm{a}$ \\
\hline & $\begin{array}{l}\text { Nuclei/nucle } \\
\text { oli }\end{array}$ & + & + & + & + & $\mathrm{n} / \mathrm{a}$ \\
\hline & Cytoplasm & $+/-$ & $+/-$ & $+/-$ & $+/-$ & $\mathrm{n} / \mathrm{a}$ \\
\hline \multirow{4}{*}{ Root } & Plastids & - & ++ & ++++ & ++ & $\mathrm{n} / \mathrm{a}$ \\
\hline & Mitochondria & - & - & - & - & $\mathrm{n} / \mathrm{a}$ \\
\hline & $\begin{array}{c}\text { Nuclei/nucle } \\
\text { oli }\end{array}$ & + & + & + & + & $\mathrm{n} / \mathrm{a}$ \\
\hline & Cytoplasm & $+/-$ & $+/-$ & $+/-$ & $+/-$ & $\mathrm{n} / \mathrm{a}$ \\
\hline
\end{tabular}


Supplemental Figure 1. Mass spectrometry results of bands present in NifH purification.

(A) Estimation of the purity of NifH preparation expressed in $N$. benthamiana. The band corresponding to the full-size NifH protein amounts for $59,05 \%$ of the total protein present in the preparation, quantified using ImageJ ${ }^{16}$. (B) Peptide mass fingerprint. Mascot search results against SwissProt database

(A)

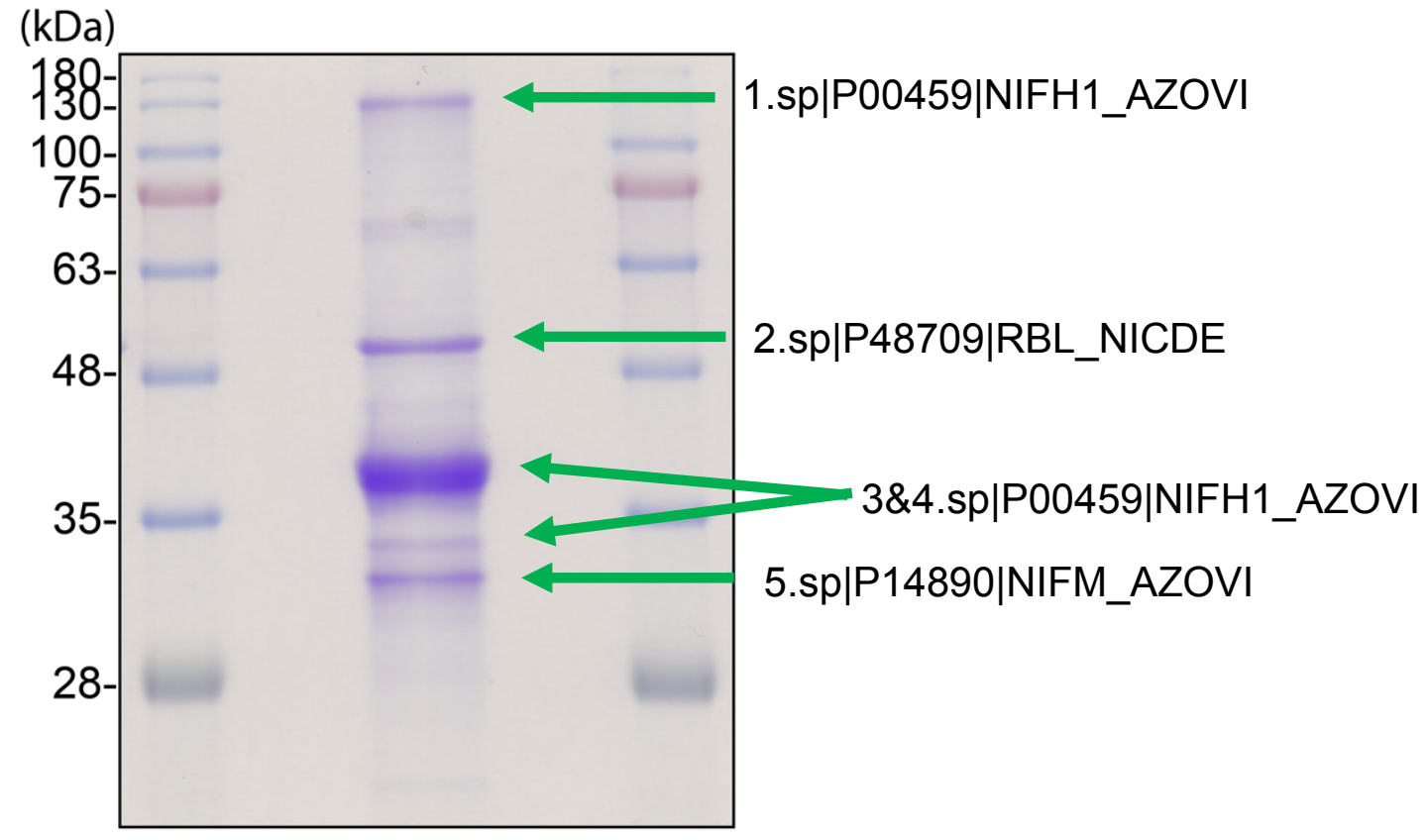


Supplemental Figure 1 (continued). Mass spectrometry results of bands present in NifH purification.

(B)

Band 1

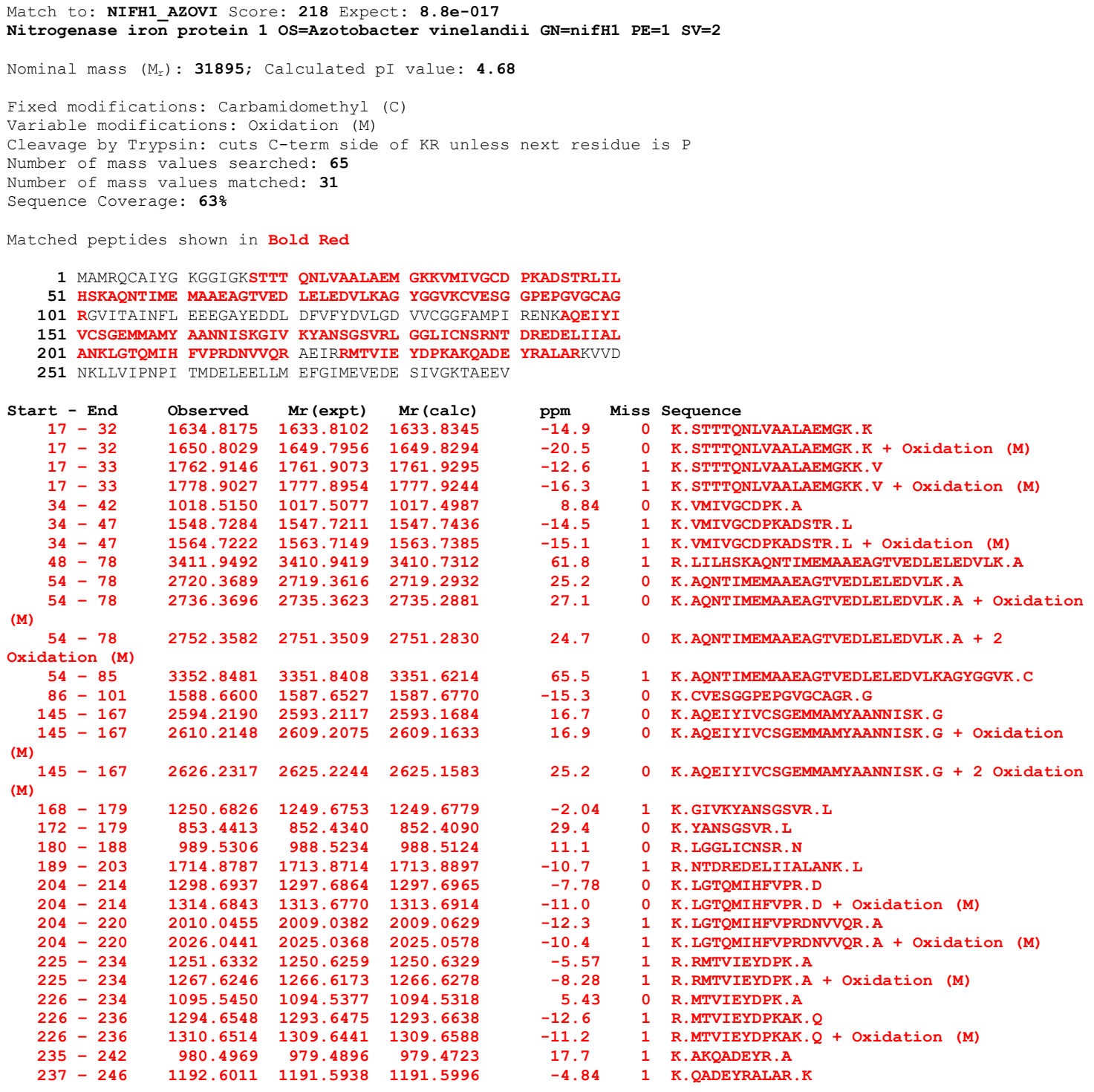

Band 2

Match to: RBL_NICDE Score: 315 Expect: $1.7 e-026$

Ribulose bisphosphate carboxylase large chain OS=Nicotiana debneyi GN=rbcL $P E=3$ SV=1

Nominal mass $\left(\mathrm{M}_{\mathrm{r}}\right)$ : 53316; Calculated $\mathrm{pI}$ value: 6.55

Fixed modifications: Carbamidomethyl (C)

Variable modifications: Oxidation (M)

Cleavage by Trypsin: cuts C-term side of KR unless next residue is $\mathrm{P}$

Number of mass values searched: 65

Number of mass values matched: 32

Sequence Coverage: $52 \%$

Matched peptides shown in Bold Red

1 MSPQTETKAS VGFKAGVKEY KLTYYTPEYQ TKDTDILAAF RVTPQPGVPP 51 EEAGAAVAAE SSTGTWTTVW TDGLTSLDRY KGRCYRIERV VGEKDQYIAY 101 VAYPLDLFEE GSVTNMFTSI VGNVFGFKAL RALRLEDLRI PPAYVKTFQC 151 PPHGIQVERD KLNKYGRPLL GCTIKPKLGL SAKNYGRAVY ECLRGGLDF' 201 KDDENVNSQP FMRWRDRFLF CAEALFKAQV ETGEIKGHYL NATAGTCEEM 251 IKRAVFAREL GVPIVMHDYL TGGFTANTSL AHYCRDNGLI LHIHRAMHAV 301 IDRQTKHHIH ERVLAKALRM SGGDHTHSGT VVGKLTGRD ITLGEVDLR 301 IDRQKNGIH 401 QFGGGTLGHP WGNAPGAVAN RVALEACVKA RNEGRDLAQE GNQIIREASK 451 WSPELAAACE VWKEIVFNFA AVDVLDK 


\begin{tabular}{|c|c|c|c|c|c|c|c|}
\hline Start & - End & Observed & $M r(\exp t)$ & $\operatorname{Mr}(\mathrm{calc})$ & ppm & Miss & Sequence \\
\hline 19 & -32 & 1826.8606 & 1825.8533 & 1825.8774 & -13.2 & 1 & K.EYKLTYYTPEYQTK.D \\
\hline 22 & -32 & 1406.6692 & 1405.6619 & 1405.6765 & -10.4 & 0 & K.LTYYTPEYQTK.D \\
\hline 22 & -41 & 2409.2219 & 2408.2146 & 2408.1900 & 10.2 & 1 & K. LTYYTPEYQTKDTDILAAFR.V \\
\hline 33 & -41 & 1021.5566 & 1020.5493 & 1020.5240 & 24.8 & 0 & K.DTDILAAFR.V \\
\hline 84 & -89 & 896.4885 & 895.4813 & 895.4334 & 53.4 & 1 & R. CYRIER.V \\
\hline 132 & -139 & 985.6098 & 984.6025 & 984.5716 & 31.4 & 1 & R. ALRLEDLR. I \\
\hline 135 & -146 & 1413.7994 & 1412.7921 & 1412.8027 & -7.50 & 1 & R. LEDLRI PPAYVK. T \\
\hline 147 & -159 & 1465.7362 & 1464.7289 & 1464.7474 & -12.6 & 0 & K. TFQGPPHGIQVER.D \\
\hline 147 & -161 & 1708.8497 & 1707.8424 & 1707.8693 & -15.7 & 1 & K. TFQGPPHGIQVERDK. L \\
\hline 165 & -177 & 1502.8287 & 1501.8214 & 1501.8439 & -15.0 & 0 & K. YGRPLLGCTIKPK.L \\
\hline 188 & -194 & 910.4882 & 909.4809 & 909.4378 & 47.4 & 0 & R.AVYECLR.G \\
\hline 195 & -213 & 2169.9834 & 2168.9761 & 2168.9797 & -1.64 & 1 & R. GGLDFTKDDENVNSQPFMR. W \\
\hline 195 & -213 & 2185.9893 & 2184.9820 & 2184.9746 & 3.39 & 1 & R.GGLDFTKDDENVNSQPFMR.W + Oxidation (M) \\
\hline 202 & -213 & 1451.6112 & 1450.6039 & 1450.6147 & -7.42 & 0 & K. DDENVNSQPFMR.W \\
\hline 216 & -227 & 1516.7455 & 1515.7382 & 1515.7544 & -10.7 & 1 & R. DRFLFCAEALFK.A \\
\hline 218 & -227 & 1245.6310 & 1244.6237 & 1244.6264 & -2.12 & 0 & R. FLFCAEALFK.A \\
\hline 237 & -253 & 1950.9048 & 1949.8975 & 1949.9087 & -5.75 & 1 & K. GHYLNATAGTCEEMIKR. A \\
\hline 237 & -253 & 1966.9031 & 1965.8958 & 1965.9037 & -3.98 & 1 & K.GHYLNATAGTCEEMIKR.A + Oxidation (M) \\
\hline 259 & -285 & 3022.6309 & 3021.6236 & 3021.4477 & 58.2 & 0 & R.ELGVPIVMHDYLTGGFTANTSLAHYCR.D \\
\hline \multirow{2}{*}{\multicolumn{8}{|c|}{ Oxidation (M) }} \\
\hline & & & & & & & \\
\hline 286 & -295 & 1187.6713 & 1186.6640 & 1186.6571 & 5.86 & 0 & R. DNGLLLHIHR. A \\
\hline 296 & -303 & 912.5040 & 911.4968 & 911.4647 & 35.2 & 0 & R. AMHAVIDR.Q \\
\hline 296 & -305 & 1168.5988 & 1167.5915 & 1167.6182 & -22.9 & 1 & R.AMHAVIDRQK .N \\
\hline 304 & -312 & 1136.6182 & 1135.6109 & 1135.5999 & 9.71 & 1 & R. QKNHGIHFR.V \\
\hline 306 & -312 & 880.4976 & 879.4903 & 879.4464 & 49.9 & 0 & K.NHGIHFR.V \\
\hline 320 & -339 & 2082.0024 & 2080.9951 & 2080.9960 & -0.43 & 1 & R.MSGGDHIHSGTVVGKLEGER.D + Oxidation (M) \\
\hline 335 & -350 & 1845.9656 & 1844.9583 & 1844.9996 & -22.4 & 1 & K. LEGERDITLGFVDLLR. D \\
\hline 340 & -350 & 1261.7120 & 1260.7047 & 1260.7078 & -2.44 & 0 & R.DITLGFVDLLR.D \\
\hline 340 & -358 & 2266.1362 & 2265.1289 & 2265.1277 & 0.52 & 1 & R.DITLGFVDLLRDDFVEQDR.S \\
\hline 432 & -446 & 1712.8455 & 1711.8382 & 1711.8601 & -12.8 & 1 & R. NEGRDLAQEGNQIIR.E \\
\hline 436 & -446 & 1256.6583 & 1255.6510 & 1255.6520 & -0.81 & 0 & R.DLAQEGNQIIR.E \\
\hline 464 & -477 & 1579.8053 & 1578.7980 & 1578.8294 & -19.8 & 0 & K.EIVFNFAAVDVLDK. - \\
\hline
\end{tabular}

Band 3

Match to: NIFH1 AzOVI Score: 152 Expect: 3.5e-010

Nitrogenase iron protein 1 OS=Azotobacter vinelandii GN=nifH1 PE=1 SV=2

Nominal mass $\left(\mathrm{M}_{r}\right)$ : 31895; Calculated $\mathrm{pI}$ value: 4.68

Fixed modifications: Carbamidomethyl (C)

Variable modifications: Oxidation (M)

Cleavage by Trypsin: cuts C-term side of KR unless next residue is $\mathrm{P}$

Number of mass values searched: 65

Number of mass values matched: 25

Sequence Coverage: $50 \%$

Matched peptides shown in Bold Red

1 MAMRQCAIYG KGGIGKSTTT QNLVAALAEM GKKVMIVGCD PKADSTRLIL 51 HSKAQNTIME MAAEAGTVED LELEDVLKAG YGGVKCVESG GPEPGVGCAG 101 RGVITAINFL EEEGAYEDDL DFVFYDVLGD VVCGGFAMPI RENKAQEIYI 151 VCSGEMMAMY AANNISKGIV KYANSGSVRL GGLICNSRNT DREDELIIAI 201 ANKLGTQMIH FVPRDNVVQR AEIRRMTVIE YDPKAKQADE YRALARKVVD 251 NKLLVIPNPI TMDELEELLM EFGIMEVEDE SIVGKTAEEV

Start - End
$17-32$
$17-33$
$17-33$
$54-78$
$54-78$
(M) $54-78$
Oxidation (M)
$54-85$
$54-85$
Oxidation (M)
$145-167$
$145-167$
(M) $145-167$
$145-179$
(M) $168-179$
$172-179$
$180-188$
$189-203$
$204-214$
$204-214$
$204-220$
$204-220$
$225-234$
$225-234$
$226-234$
$226-236$
$235-242$
$237-246$

\begin{tabular}{|c|c|c|}
\hline ed & $\mathrm{xpt}$ ) & alc) \\
\hline 1634.7944 & 1633.7871 & 1633.8345 \\
\hline 1762.8884 & 1761.8811 & 1761.9295 \\
\hline 1778.8793 & 1777.8720 & 1777.9244 \\
\hline 2720.2229 & 2719.2156 & 2719.2932 \\
\hline 2736.2214 & 2735.2141 & 2735.2881 \\
\hline 52. & 2751 & 2751 . \\
\hline & 335 & 33 \\
\hline 3 & 3383 & 3383. \\
\hline & & \\
\hline 261 & 260 & 2609 \\
\hline 3 & 0 & \\
\hline 12 & 124 & 1249. \\
\hline 853.38 & 852.3 & 852. \\
\hline 989.4786 & 988.4714 & 24 \\
\hline 1714.8528 & 1713 & 1713. \\
\hline 1298 & 1297. & 1297. \\
\hline 1314.6600 & 1313.6527 & 1313. \\
\hline & 2009.0098 & 2009.0629 \\
\hline .0066 & 2024 & 2025 . \\
\hline 1251.6139 & 1250 & 1250. \\
\hline 1267.6006 & 1266.5933 & 1266.6278 \\
\hline 1095.5027 & 1094. & 1094 \\
\hline 129 & 129 & 1293.6 \\
\hline 980.4 & 4390 & \\
\hline & & \\
\hline
\end{tabular}

$\begin{array}{cc}\text { ppm } & \text { M } \\ -29.0 & 0 \\ -27.4 & 1 \\ -29.4 & 1 \\ -28.5 & 0 \\ -27.0 & 0 \\ -26.8 & 0 \\ -27.3 & 1 \\ -26.7 & 1 \\ & \\ -29.2 & 0 \\ -28.3 & 0 \\ & \\ -31.7 & 0 \\ & \\ -15.8 & 1 \\ -40.5 & 0 \\ -41.5 & 0 \\ -25.8 & 1 \\ -27.6 & 0 \\ -29.5 & 0 \\ -26.4 & 1 \\ -28.9 & 1 \\ -21.0 & 1 \\ -27.2 & 1 \\ -33.2 & 0 \\ -28.8 & 1 \\ -34.0 & 1 \\ -23.2 & 1 \\ & \end{array}$

Miss Sequence

K. STTTQNLVAALAEMGK . K

K. STTTQNLVAALAEMGKK.V

K.STTTQNLVAALAEMGKK.V + Oxidation (M)

K. AQNT IMEMAAEAGTVEDLELEDVLK. A

K.AQNTIMEMAAEAGTVEDLELEDVLK.A + Oxidation

K. AQNTIMEMAAEAGTVEDLELEDVLK.A +2

K. AONT IMEMAAEAGTVEDLELEDVLKAGYGGVK. C

K. AQNTIMEMAAEAGTVEDLELEDVLKAGYGGVK.C + 2

K. AQEIYIVCSGEMMAMYAANNISK . G

K.AQEIYIVCSGEMMAMYAANNISK.G + Oxidation

K.AQEIYIVCSGEMMAMYAANNISK.G + 2 Oxidation

K. GIVKYANSGSVR. I

K. YANSGSVR. I

R. LGGLICNSR.N

R. NTDREDELIIALANK. I

K. LGTQMIHFVPR. D

K.LGTOMIHFVPR.D + Oxidation (M)

K. LGTQMIHFVPRDNVVQR. A

K. LGTOMIHFVPRDNVVOR. A + Oxidation (M)

R. RMTVIEYDPK.A

R. RMTVIEYDPK.A + Oxidation (M)

R. MTVIEYDPK. A

R.MTVIEYDPKAK. $Q$

K. AKQADEYR. A

K. QADEYRALAR. K 
Band 4

Match to: NIFH1_AzOVI Score: 173 Expect: 2.8e-012

Nitrogenase iron protein 1 OS=Azotobacter vinelandii $G N=n i f H 1 \quad P E=1 \quad S V=2$

Nominal mass $\left(\mathrm{M}_{\mathrm{r}}\right)$ : 31895; Calculated $\mathrm{pI}$ value: 4.68

Fixed modifications: Carbamidomethyl (C)

Variable modifications: Oxidation (M)

Cleavage by Trypsin: cuts C-term side of KR unless next residue is $\mathrm{P}$

Number of mass values searched: 65

Number of mass values matched: 28

Sequence Coverage: 59\%

Matched peptides shown in Bold Red

1 MAMRQCAIYG KGGIGKSTTT QNLVAALAEM GKKVMIVGCD PKADSTRLII 51 HSKAQNTIME MAAEAGTVED LELEDVLKAG YGGVKCVESG GPEPGVGCAG 101 RGVITAINEL EEEGAYEDDL DFVFYDVLGD VVCGGFAMPI RENKAQEIYI 151 VCSGEMMAMY AANNISKGIV KYANSGSVRL GGLICNSRNT DREDELIIAI 201 ANKLGTOMIH FVPRDNVVOR AEIRRMTVIE YDPKAKOADE YRALARKVVD 251 NKLLVIPNPI TMDELEELIM EFGIMEVEDE SIVGKTAEEV

\begin{tabular}{|c|c|c|c|c|}
\hline cart & - End & Observed & $M r(\exp t)$ & $\mathrm{Mr}$ (ca \\
\hline 17 & -32 & 1634.8141 & 1633.8068 & 1633.83 \\
\hline 17 & -32 & 1650.8110 & 1649.8037 & 1649.8 \\
\hline 17 & -33 & 1762.9119 & 1761.9046 & 1761.92 \\
\hline 17 & -33 & 1778.9048 & 1777.8975 & 1777. \\
\hline 34 & -42 & 1018.5162 & 1017.5089 & 1017.4 \\
\hline 34 & -47 & 1548.7299 & 1547.7226 & 1547 \\
\hline 34 & -47 & 1564.7418 & 1563.7345 & 1563. \\
\hline 54 & -78 & 2720.3606 & 2719.3533 & 2719 \\
\hline 54 & -78 & 2736.3652 & 2735.3579 & 2735 \\
\hline \multicolumn{5}{|l|}{ (M) } \\
\hline 54 & -78 & 2752.3560 & 2751.3487 & 2751 \\
\hline \multicolumn{5}{|c|}{ Oxidation (M) } \\
\hline 54 & -85 & 3352.8301 & 3351.8228 & 335 \\
\hline 54 & -85 & 3368.8384 & 3367.8311 & 3367 \\
\hline \multicolumn{5}{|c|}{ Oxidation (M) } \\
\hline 86 & -101 & 1588.6731 & 1587.6658 & 1587 \\
\hline 145 & - 167 & 2594.2185 & 2593.2112 & 2593 \\
\hline 145 & -167 & 2610.2239 & 2609.2166 & 2600 \\
\hline \multicolumn{5}{|l|}{ (M) } \\
\hline 145 & -167 & 2626.2085 & 2625.2012 & \\
\hline \multicolumn{5}{|l|}{ (M) } \\
\hline 172 & -179 & 853.4465 & 852.4392 & 852 \\
\hline 180 & -188 & 989.5368 & 988.5295 & 988. \\
\hline 189 & -203 & 1714.8777 & 1713.8704 & 1713.8 \\
\hline 204 & -214 & 1298.6947 & 1297.6874 & 1297. \\
\hline 204 & -214 & 1314.6890 & 1313.6817 & 1313. \\
\hline 204 & -220 & 2010.0569 & 2009.0496 & 2009.0 \\
\hline 204 & -220 & 2026.0487 & 2025.0414 & 2025 . \\
\hline 225 & -234 & 1251.6360 & 1250.6287 & 1250. \\
\hline 225 & -234 & 1267.6428 & 1266.6355 & 1266. \\
\hline 226 & -234 & 1095.5449 & 1094.5376 & 1094. \\
\hline 226 & -236 & 1294.6613 & 1293.6540 & 1293. \\
\hline 237 & -246 & 1192.6123 & 1191.6050 & 1191. \\
\hline
\end{tabular}

\begin{tabular}{|c|c|c|}
\hline ppm & Miss & Sequence \\
\hline-16.9 & 0 & K. STTTQNLVAALAEMGK.K \\
\hline-15.6 & 0 & K.STTTQNLVAALAEMGK.K + Oxidation (M) \\
\hline-14.1 & 1 & K. STTTQNLVAALAEMGKK.V \\
\hline-15.1 & 1 & K.STTTQNLVAALAEMGKK.V + Oxidation (M) \\
\hline 10.0 & 0 & K. VMIVGCDPK. A \\
\hline-13.6 & 1 & K. VMIVGCDPKADSTR. L \\
\hline-2.56 & 1 & K.VMIVGCDPKADSTR.I + Oxidation (M) \\
\hline 22.1 & 0 & K. AQNT IMEMAAEAGTVEDLELEDVLK. A \\
\hline 25.5 & 0 & K.AQNTIMEMAAEAGTVEDLELEDVLK.A + Oxidation \\
\hline 23.9 & 0 & K. AQNTIMEMAAEAGTVEDLELEDVLK.A +2 \\
\hline 60.1 & 1 & K. AQNTIMEMAAEAGTVEDLELEDVLKAGYGGVK.C \\
\hline 63.8 & 1 & K. AQNTIMEMAAEAGTVEDLELEDVLKAGYGGVK.C + \\
\hline-7.04 & 0 & K. CVESGGPEPGVGCAGR.G \\
\hline 16.5 & 0 & K. AQEIYIVCSGEMMAMYAANNISK. G \\
\hline 20.4 & 0 & K.AQEIYIVCSGEMMAMYAANNISK.G + Oxidation \\
\hline 16.4 & 0 & K.AQEIYIVCSGEMMAMYAANNISK.G +2 Oxidation \\
\hline 35.5 & 0 & K. YANSGSVR. L \\
\hline 17.3 & 0 & R. LGGLICNSR.N \\
\hline-11.2 & 1 & R. NTDREDELIIALANK. L \\
\hline-7.01 & 0 & K. LGTQMIHFVPR. D \\
\hline-7.39 & 0 & K.LGTQMIHFVPR.D + Oxidation (M) \\
\hline-6.61 & 1 & K. LGTQMIHFVPRDNVVQR. A \\
\hline-8.10 & 1 & K.LGTQMIHFVPRDNVVQR.A + Oxidation (M) \\
\hline-3.33 & 1 & R. RMTVIEYDPK.A \\
\hline 6.09 & 1 & R.RMTVIEYDPK.A + Oxidation (M) \\
\hline 5.34 & 0 & R.MTVIEYDPK.A \\
\hline-7.59 & 1 & R.MTVIEYDPKAK. $Q$ \\
\hline 4.56 & 1 & K. QADEYRALAR.K \\
\hline
\end{tabular}

Band 5

Match to: NIFM_Azovi Score: 70 Expect: 0.055

Putative peptid̄yl-prolyl cis-trans isomerase NifM OS=Azotobacter vinelandii $\mathrm{GN}=\mathrm{nifM} \mathrm{PE}=3 \mathrm{SV}=1$

Nominal mass $\left(\mathrm{M}_{\mathrm{r}}\right)$ : 33181; Calculated pI value: 5.45

Fixed modifications: Carbamidomethyl (C)

Variable modifications: Oxidation (M)

Number of mass values searched: 65

Number of mass values matched: 10

Sequence Coverage: $39 \%$

Matched peptides shown in Bold Red

1 MASERLADGD SRYYLLKVAH EQFGCAPGEL SEDQLQQADR IIGRQRHIED

51 AVLRSPDAIG VVIPPSQLEE AWAHIASRYE SPEALQQALD AQALDAAGMR

101 AMLARELRVE AVLDCVCAGL PEISDTDVSL YYFNHAEQFK VPAQHKAHIL

151 VTINEDFPEN TREAARTRIE TILKRLRGKP ERFAEOAMKH SECPTAMOGG

201 LLGEVVPGTL YPELDACLFQ MARGELSPVL ESPIGFHVLY CESVSPARQL

251 TLEEILPRLR DRLQLRQRKA YQRKWLVCLL QQNATLENLA HG

\begin{tabular}{|c|c|c|c|c|c|c|c|}
\hline Start & - End & Observed & Mr (expt) & $\operatorname{Mr}(\mathrm{calc})$ & ppm & Miss & Sequence \\
\hline 6 & -17 & 1413.7352 & 1412.7279 & 1412.7299 & -1.43 & 1 & R. LADGDSRYYLLK.V \\
\hline 45 & -54 & 1236.6891 & 1235.6818 & 1235.6734 & 6.78 & 1 & R. QRHIEDAVLR.S \\
\hline 47 & -54 & 952.5436 & 951.5363 & 951.5138 & 23.7 & 0 & R. HIEDAVLR.S \\
\hline 55 & -78 & 2543.3379 & 2542.3306 & 2542.3180 & 4.98 & 0 & R. SPDAIGVVIPPSQLEEAWAHIASR.Y \\
\hline 79 & -100 & 2348.1182 & 2347.1109 & 2347.1114 & -0.19 & 0 & R. YESPEALQQALDAQALDAAGMR . A \\
\hline 79 & -100 & 2364.1147 & 2363.1074 & 2363.1063 & 0.48 & 0 & R. YESPEALQQALDAQALDAAGMR.A + Oxidation (M) \\
\hline 79 & -105 & 2922.4561 & 2921.4488 & 2921.4011 & 16.3 & 1 & R. YESPEALQQALDAQALDAAGMRAMLAR.E +2 \\
\hline \multicolumn{8}{|c|}{ Oxidation (M) } \\
\hline 167 & -174 & 973.6284 & 972.6211 & 972.5968 & 25.1 & 1 & R. TRIETILK.R \\
\hline 224 & -248 & 2743.4026 & 2742.3953 & 2742.3687 & 9.71 & 0 & R. GELSPVLESPIGFHVLYCESVSPAR. $Q$ \\
\hline 249 & -258 & 1211.7137 & 1210.7064 & 1210.6921 & 11.8 & 0 & R. QLTLEEILPR.L \\
\hline
\end{tabular}


Supplemental Figure 2. Determination of N-terminal sequence of TwinStrep-NifH using the standard Edman degradation method.

Blank, standard and first six amino acids were determined ( $\mathrm{mV}$ vs retention time in mins).
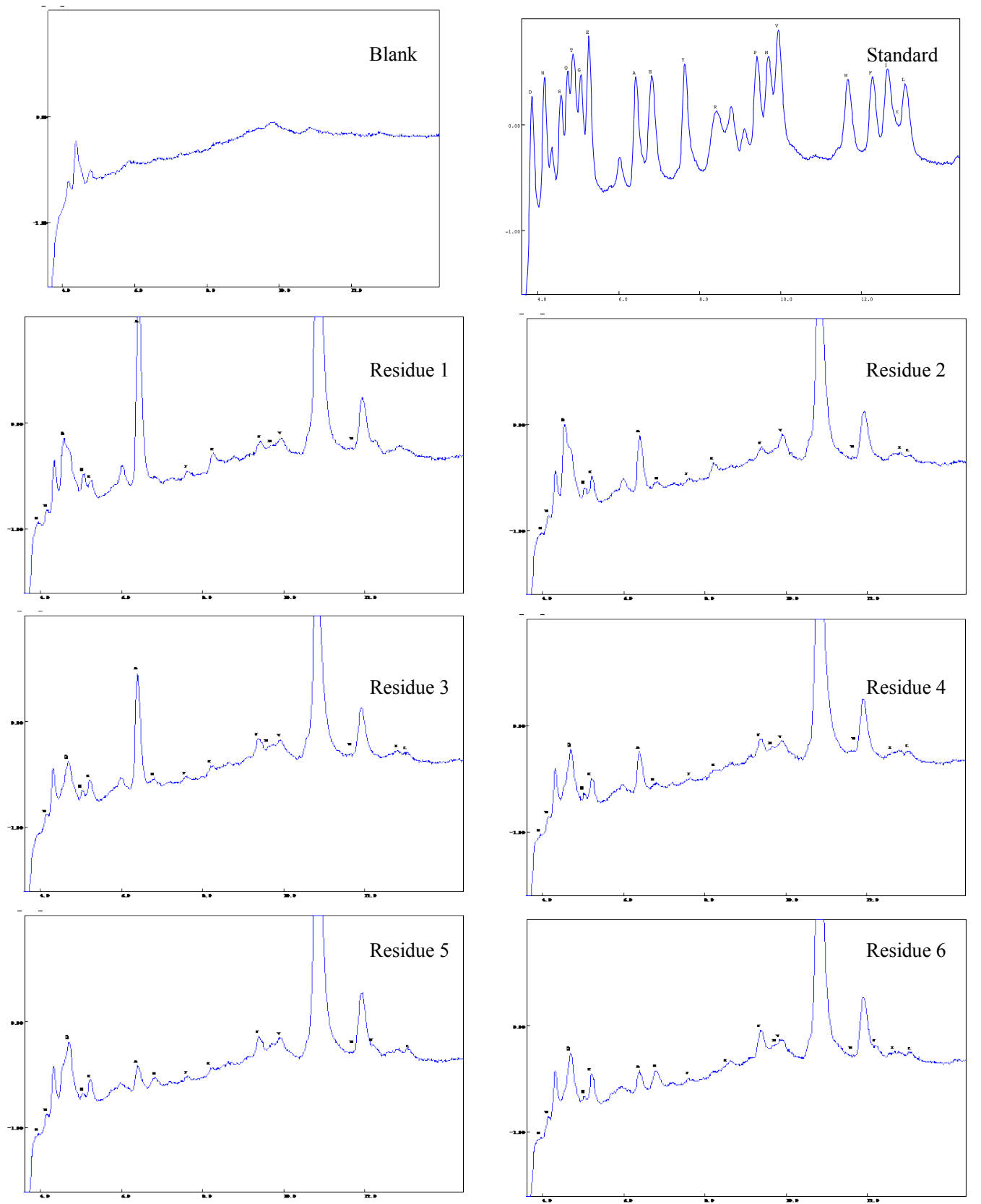

\begin{tabular}{|c|c|c|c|c|c|c|}
\hline AA step & 1 & 2 & 3 & 4 & 5 & 6 \\
\hline 1. $\mathrm{AA}$ & $\mathbf{A}$ & $\mathbf{S}$ & $\mathbf{A}$ & $?$ & $\mathrm{Q} / \mathrm{S}$ & $\mathbf{H}$ \\
\hline 2. $\mathrm{AA}$ & $\mathrm{S}$ & $\mathrm{V}$ & $\mathrm{P}$ & Q & $\mathrm{H}$ & \\
\hline
\end{tabular}

Green: TP

Red: TwinStrep Blue: NifH

Black: cloning scars

MAAAVSTVGAINRAPLSLNGSGSGAVSAPASTFLGKKVVTVSRFAQSNKKSNGSFKVLAASAWSHPQFEK GGGSGGGSGGSAWSHPQFEKSSMAMRQCAIYGKGGIGKSTTTQNLVAALAEMGKKVMIVGCDPKADST RLILHSKAQNTIMEMAAEAGTVEDLELEDVLKAGYGGVKCVESGGPEPGVGCAGRGVITAINFLEEEGAYED DLDFVFYDVLGDVVCGGFAMPIRENKAQEIYIVCSGEMMAMYAANNISKGIVKYANSGSVRLGGLICNSRN TDREDELIIALANKLGTQMIHFVPRDNVVQRAEIRRMTVIEYDPKAKQADEYRALARKVVDNKLLVIPNPIT MDELEELLMEFGIMEVEDESIVGKTAEEV 
Supplemental Figure 3. NifM and NifU co-elution of with Strep-tagged NifH.

Strep-tactin purification procedure of $A$. vinelandii $\mathrm{NifH}$ expressed in leaves of $N$. benthamiana. $\mathrm{CFE}$, cell-free extract (soluble fraction following centrifugation and filtering of disrupted leaf tissue); FT, Strep-tactin flow-through fraction; W, wash fraction; E, biotin-eluted fraction; $\mathrm{E}_{\mathrm{c}}$, concentrated biotin-eluted fraction. (A) Western blot using antibodies against NifM. (B) Western blot using antibodies against NifU. (C) Western blot using antibodies against NifS. Arrows point at the correct size of the proteins, the higher mobility bands in NifS blot correspond to the signal from $\alpha$-NifM wich was developed before $\alpha$-NifS incubation.

A

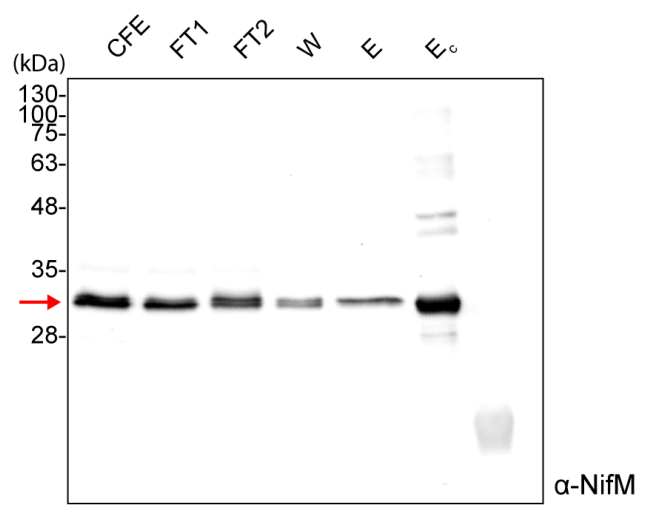

C

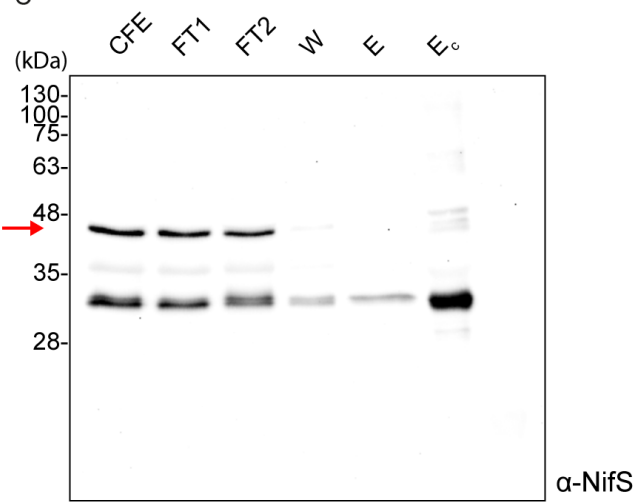

B

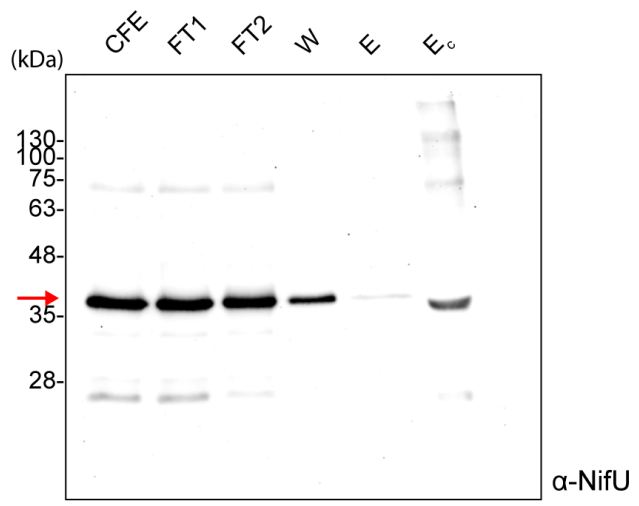


Supplemental Figure 4. Gene expression of TOC and TIC family members in A. thaliana and O. sativa.

Data was retrieved from the Plant eFP browser, showing $\log _{2}$ ratio from microarray transcriptomics of Arabidopsis thaliana (A) and Oryza sativa (B) tissues ${ }^{17}$. (C) Homologs of A. thaliana TOC and TIC family members in O. sativa ssp. japonica. In the case of Toc132-Toc120 and Toc33-Toc34, the same one homolog was retrieved respectively.

(A)

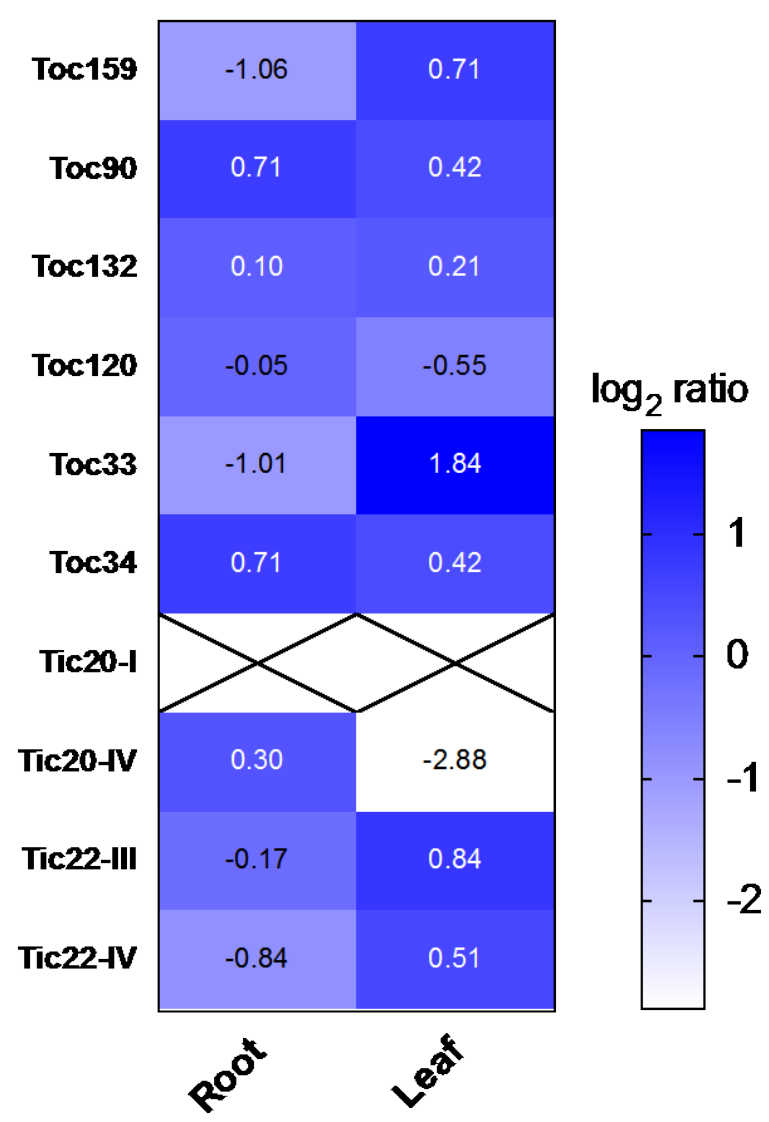


(B)

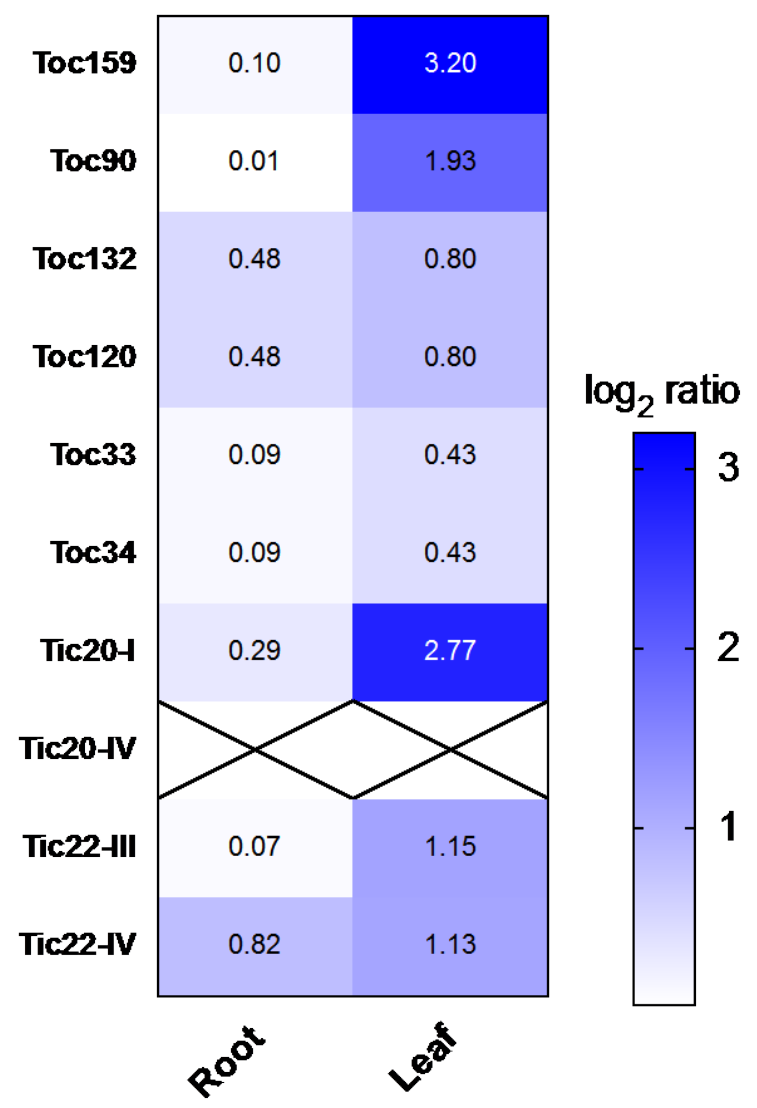

(C)

\begin{tabular}{ccc}
\hline Protein & Arabidopsis thaliana & Oryza sativa \\
\hline Toc159 & AT4G02510 & Os05g0151400 \\
Toc90 & AT5G20300 & Os12g0197400 \\
Toc132 & AT2G16640 & Os10g0548800 \\
Toc120 & AT3G16620 & Os10g0548800 \\
Toc33 & AT1G02280 & Os03g0240500 \\
Toc34 & AT5G05000 & Os03g0240500 \\
Tic20-I & AT1G04940 & Os07g0568500 \\
Tic20-IV & AT4G03320 & - \\
Tic22-III & AT3G23710 & Os07g0290800 \\
Tic22-IV & AT4G33350 & Os06g0213200 \\
\hline
\end{tabular}


Supplemental Figure 5. Biological context of A. thaliana genes containing selected Transit Peptides.

(A) Schematic representation of the metabolic pathways in which genes $A T 3 G 54890$ (AtCAB6), AT4G32770 (AtTOCC), AT2G39730 (AtRCA), AT5G16390 (AtBCCP1) and AT2G41220 $\left(A t\right.$ GLTB2) are involved. Illustration obtained from MapMan ${ }^{18}$ overview of plant metabolism. (B) Gene Ontology Annotations.

(A)
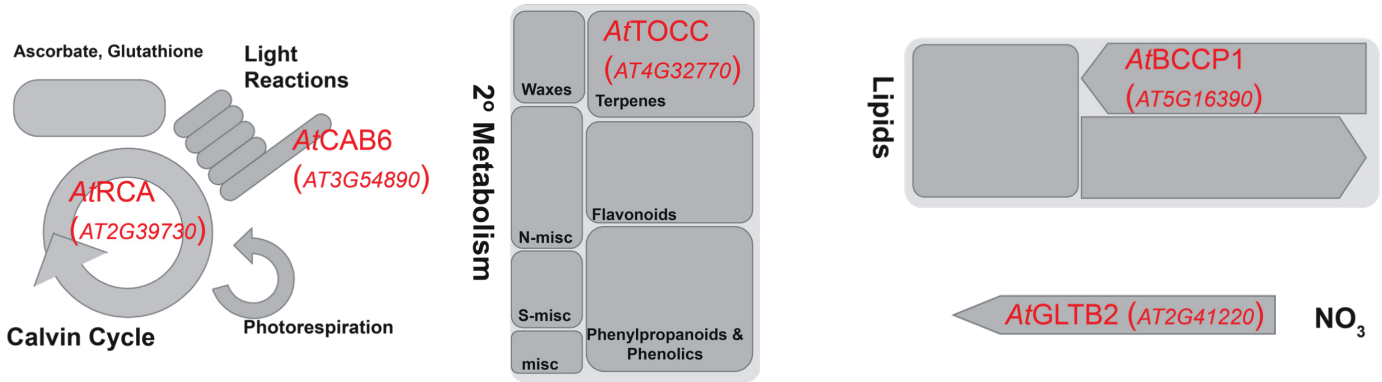

(B)

\section{AtCAB6}

(AT3G54890) $\begin{array}{cc}\text { GO Biological } & \begin{array}{c}\text { Photosynthesis, light } \\ \text { harvesting in } \\ \text { Process }\end{array} \\ \text { photosystem I }\end{array}$
AtRCA

(AT2G39730)

Response to light stimulus, jasmonic acid

Chloroplast (envelope, stroma, thylakoid)
AtTOCC

(AT4G32770)

Vitamin E biosynthetic process
AtBCCP1m

(AT5G16390)
AtGLTB2

(AT2G41220)
GO Cellular
Component

GO Molecular

Function

\begin{abstract}
Chloroplast
(envelope,

thylakoid)
\end{abstract}

Chlorophyll binding
ADP and ATP

binding, ribulose-1,5-

bisphosphate carboxylase/oxygenas e activator activity
Tocopherol cyclase activity
Fatty acid biosynthetic process
Ammonia assimilation cycle, glutamate biosynthetic process
Chloroplast (envelope, stroma)
Chloroplast (envelope, stroma) (envelope, thylakoid)

\section{Biotin binding, acetyl-CoA} carboxylase activity
Glutamate synthase (ferredoxin) activity 
Supplemental Figure 6. Isolated protoplasts imaged using light microscopy.

Isolated protoplasts from (A) Arabidopsis thaliana aerial part, (B) roots and (C) Oryza sativa stem and sheath tissue were imaged with a Zeiss AxioPhot Microscope (Zeiss) equipped with a Plan-Neofluar 40X/0.75 objective. Scale bar represents (A) $40 \mu \mathrm{m}$ or (B-C) $20 \mu \mathrm{m}$.

(A)

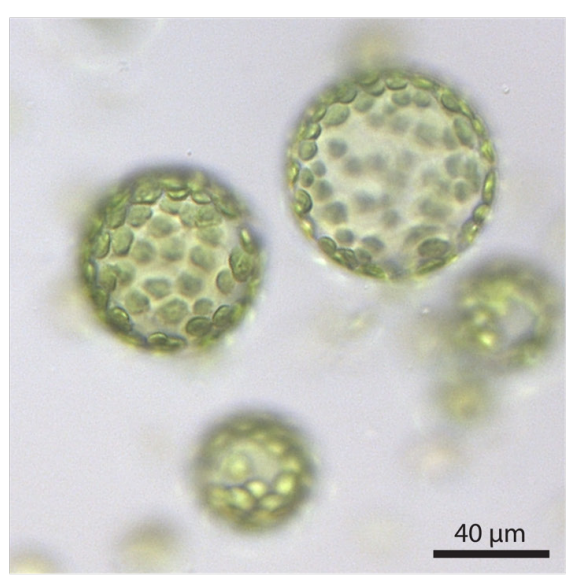

(B)

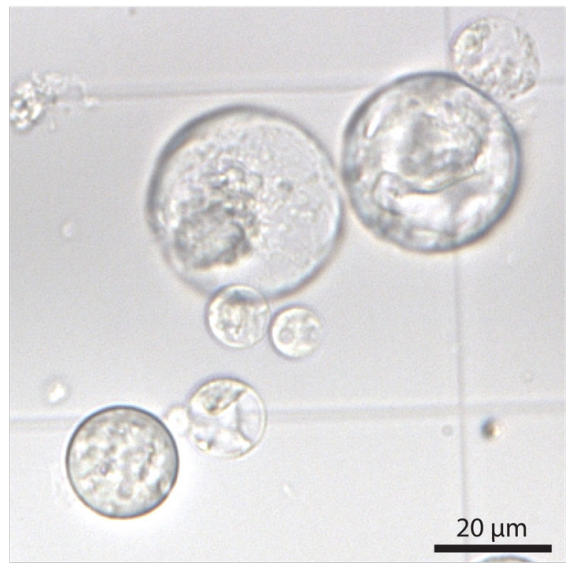

(C)

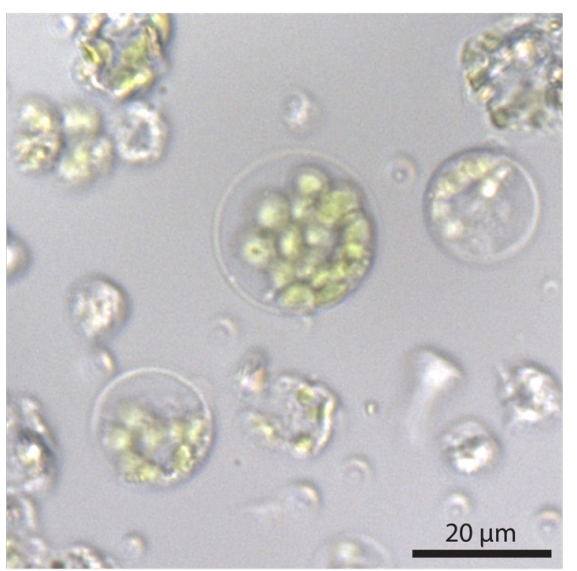


Supplemental Figure 7. Immunogold Transmission Electron Microscopy images treated with polyclonal antibody.

Immunogold labelling of eGFP in (A-D) proplastids of rice callus cells, $(\mathbf{E}-\mathbf{H})$ chloroplast of rice leaf cells and (I-L) plastids of rice root cells using a GFP polyclonal antibody (diluted 1:250). A, E, I Wild-type cells. B, F, J AtmCAB6 ${ }_{\mathrm{TP}}-\mathrm{eGFP}$. C, G, K AtmRCA ${ }_{\mathrm{TP}}-\mathrm{eGFP}$. D, H, L AtmTOCC $\mathrm{TP}_{\mathrm{TP}}$ eGFP. i-vi: Transmission electron microscope (TEM) images. (CW: cell wall, m: mitochondria, $\mathrm{N}$ : nucleus, Nu: nucleolus, p: plastid like, St: starch, Ch: chloroplast, p-c: plastid showing an isolated cytoplasmic region; bars $=500 \mathrm{~nm}$; gold particle size $=15 \mathrm{~nm}$ )

(A)
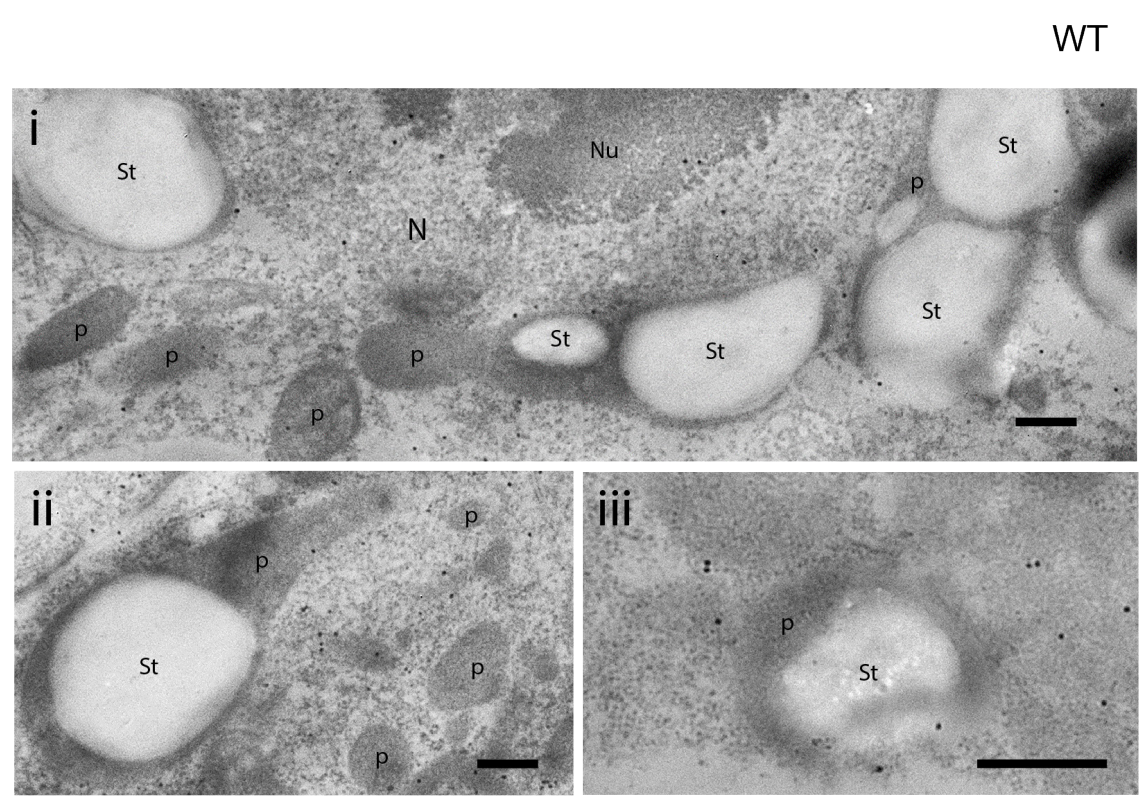

(B)
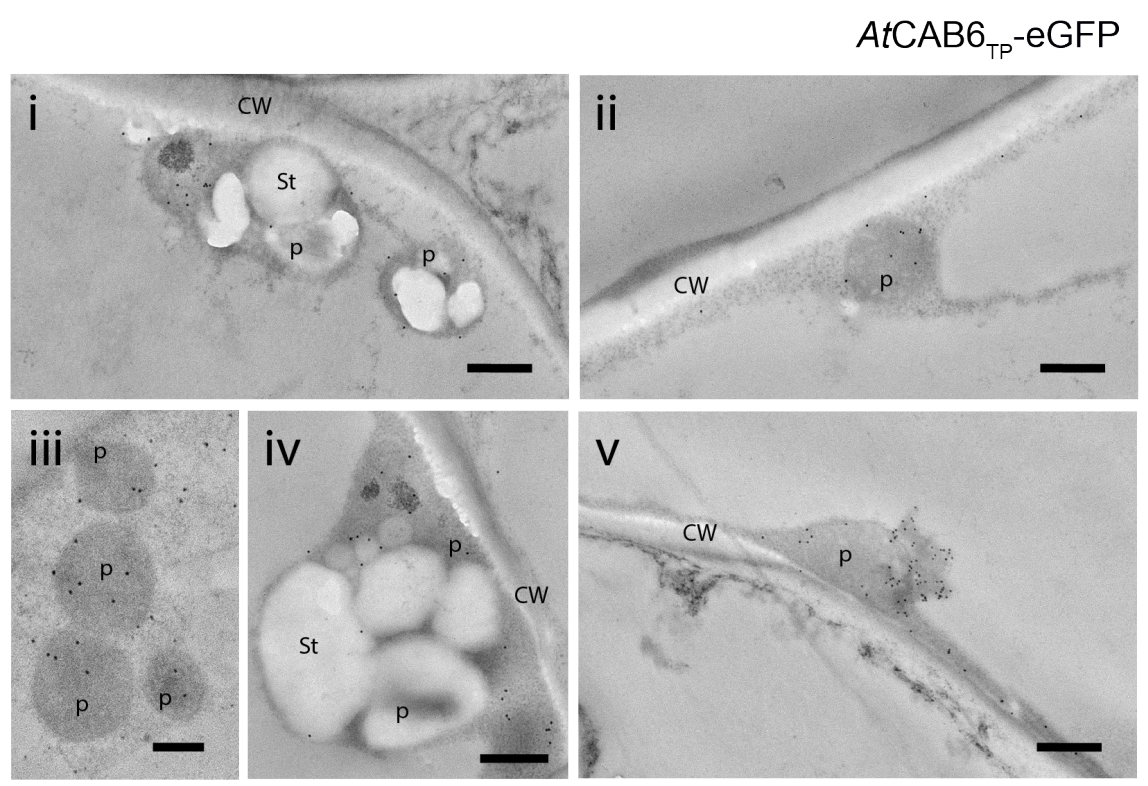
(C)

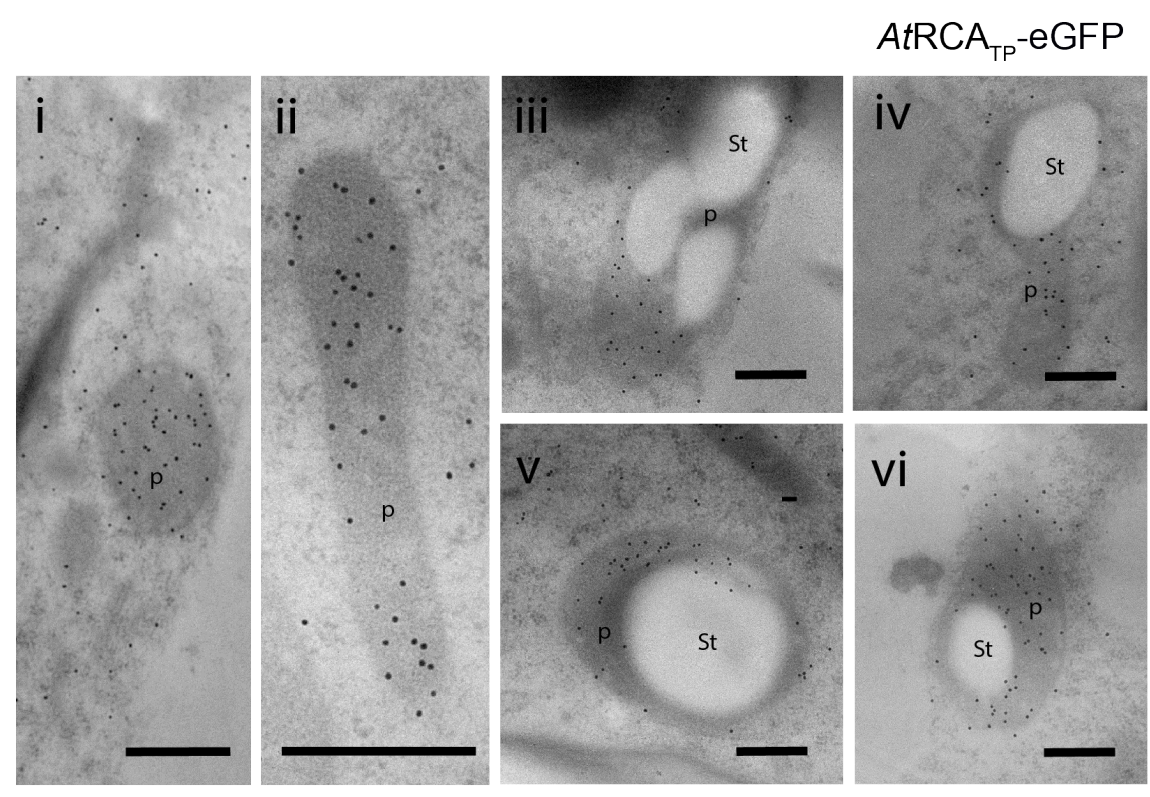

(D)

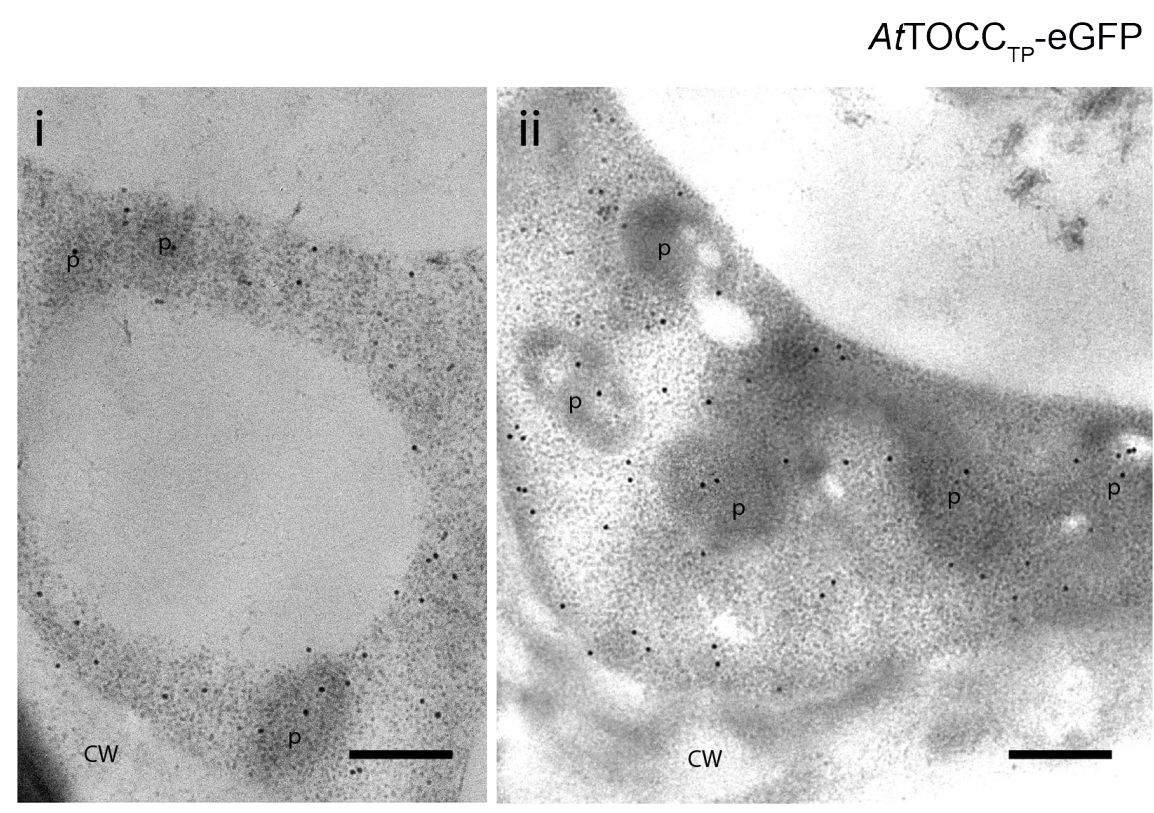


(E)

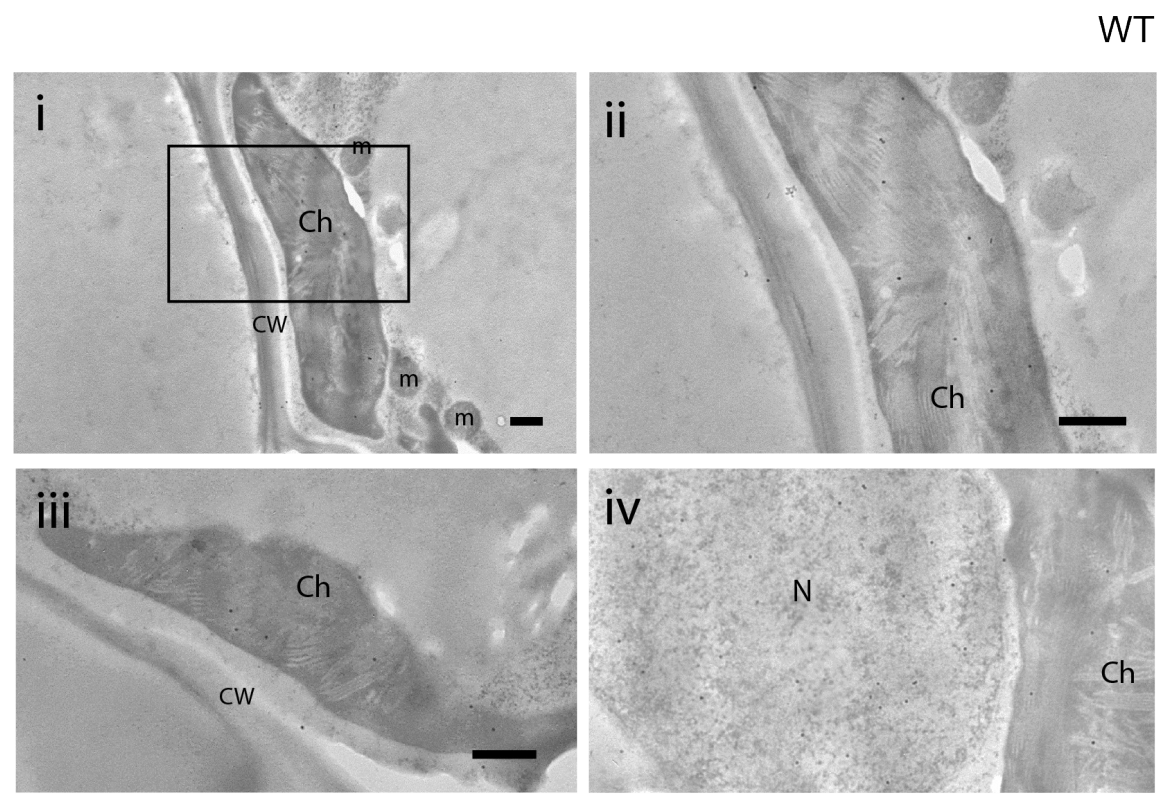

(F)

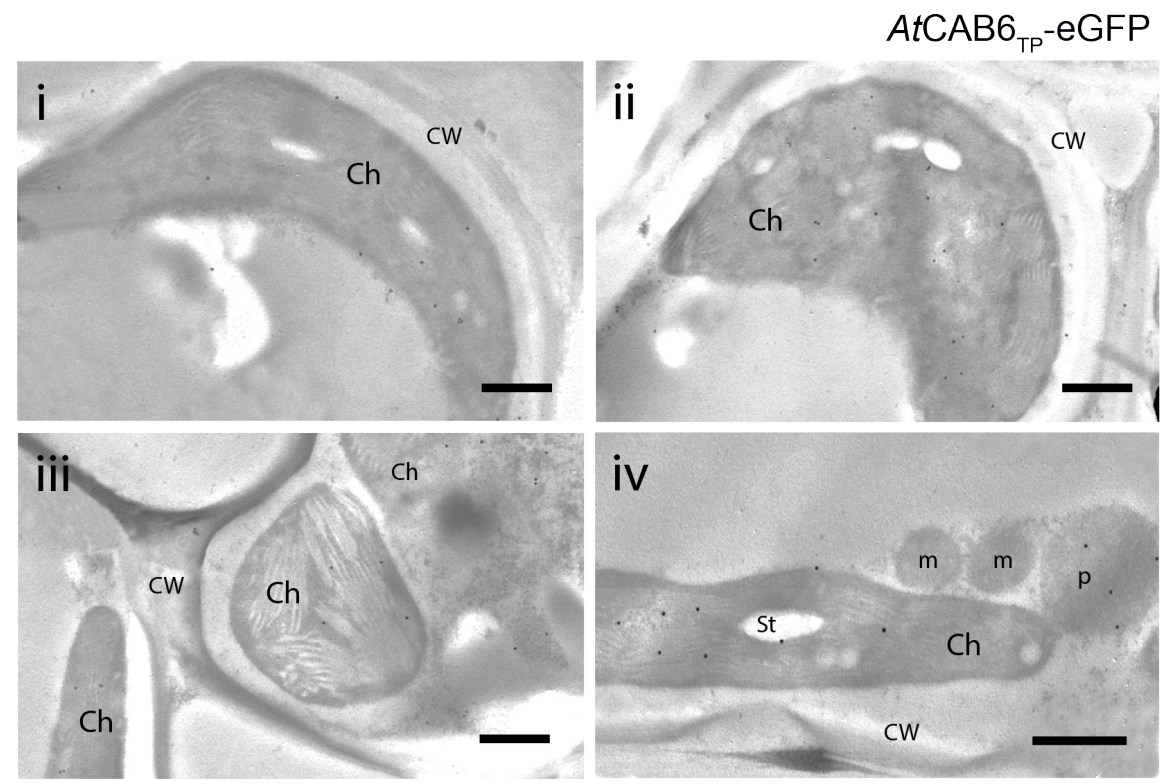


(G)

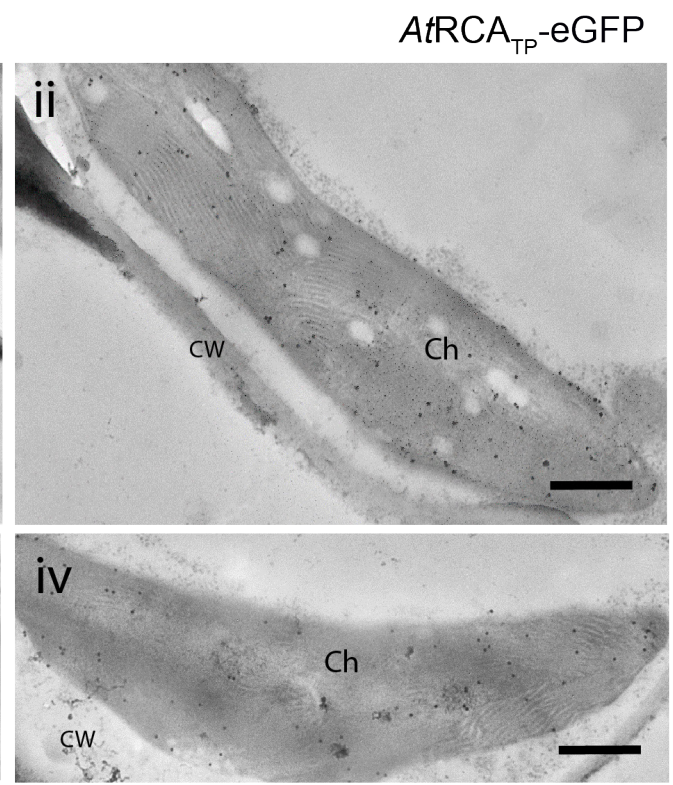

(H)

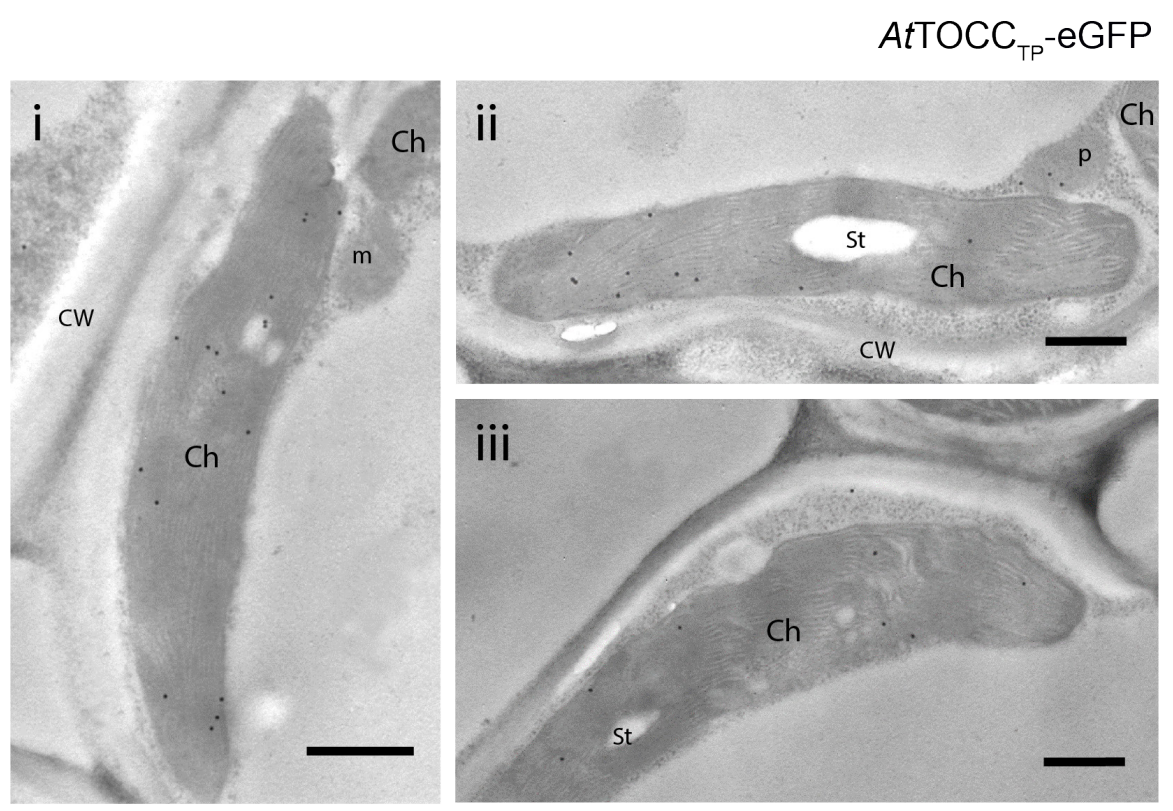


(I)
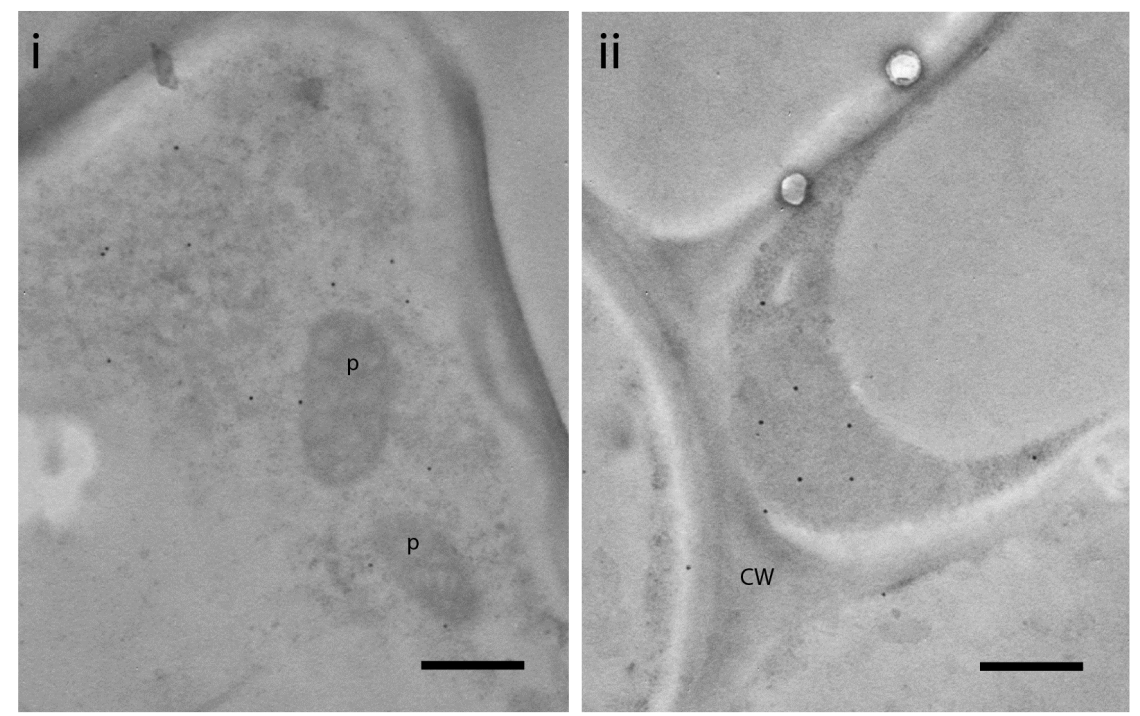

(J)
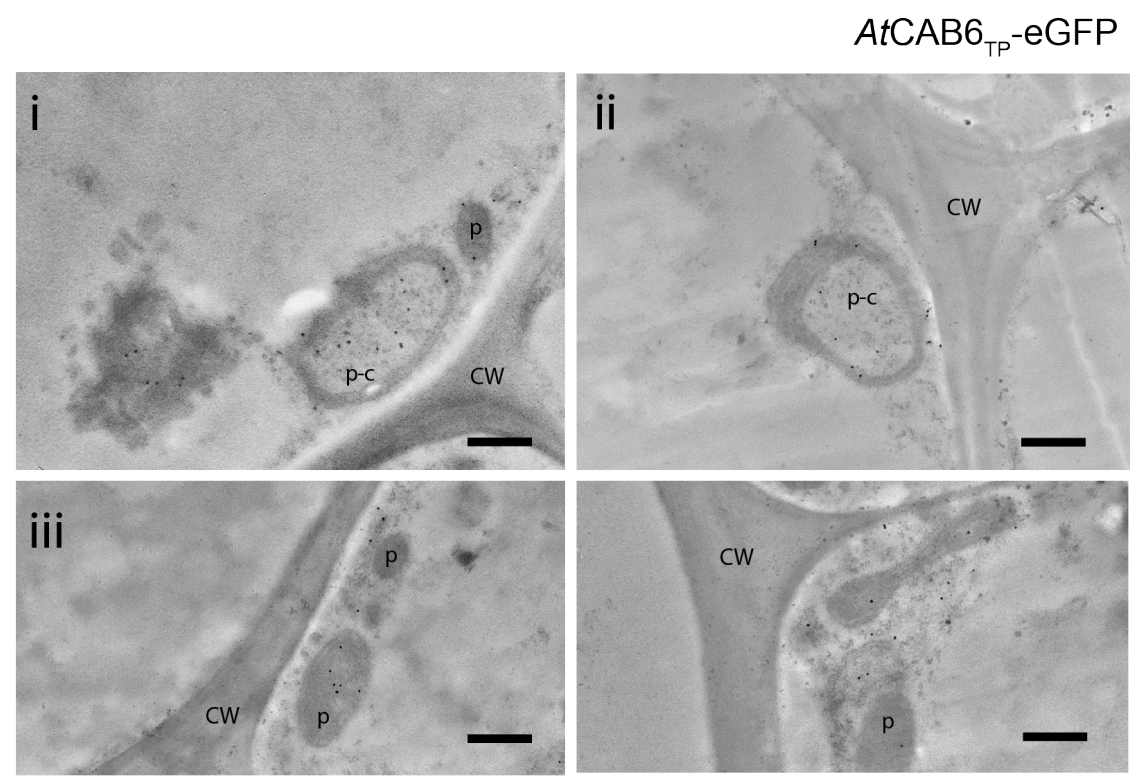
(K)
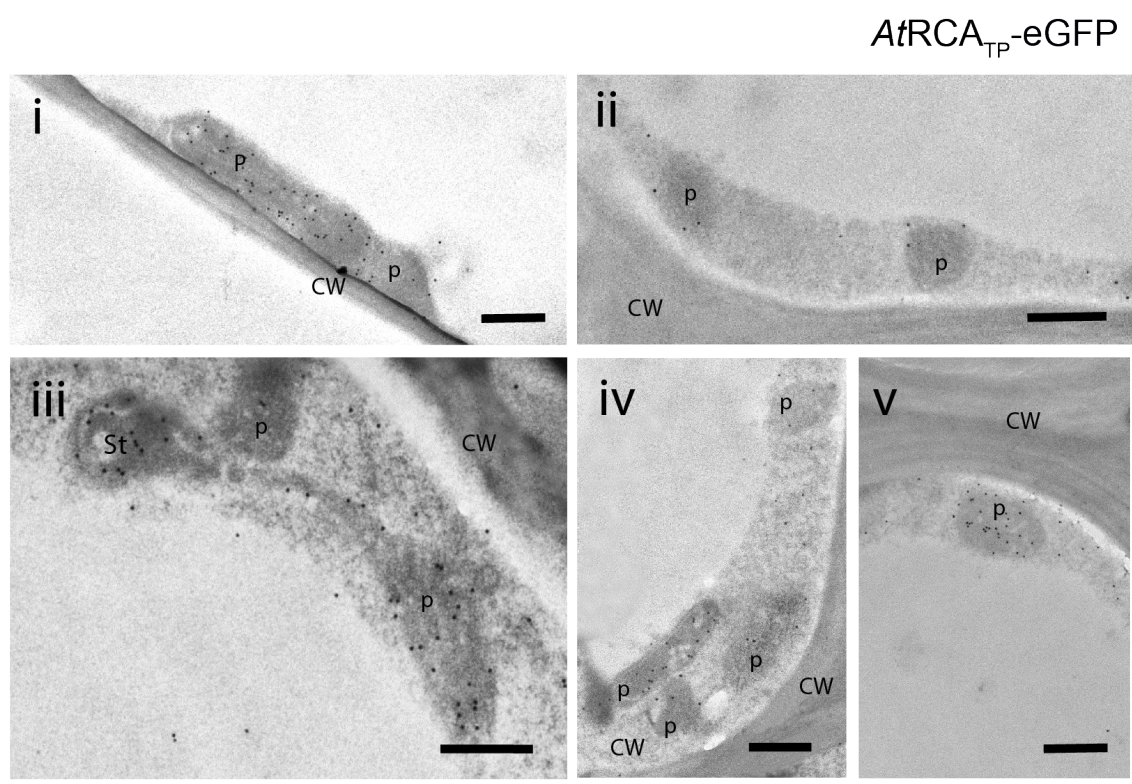

(L)

$$
\text { AtTOCC }_{\mathrm{TP}}-\mathrm{eGFP}
$$
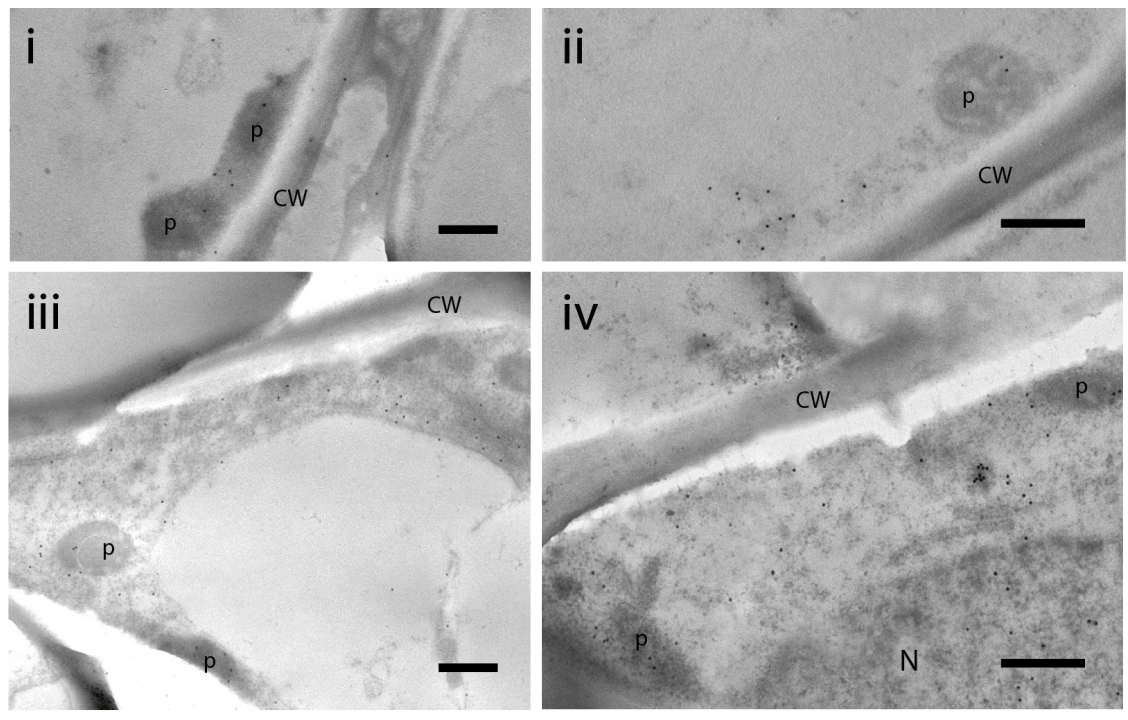
Supplemental Figure 8. Confocal laser scanning microscopy images of wild-type and transgenic lines rice leaves.

Confocal laser scanning microscopy images of wild- type (WT) rice leaves and leaves from transgenic lines transformed with AtmCAB6 ${ }_{\mathrm{TP}}-\mathrm{eGFP}, A t \mathrm{mRCA} \mathrm{TP}_{\mathrm{T}} \mathrm{eGFP}$ and $A t \mathrm{mTOCC} \mathrm{TP}_{\mathrm{TP}} \mathrm{eGFP}$ fusions. The four panels show the individual signals for eGFP (a) chlorophyll autofluorescence (b), transmitted light (c) and the overlap of eGFP and chlorophyll autofluorescence (d). Scale bars, $20 \mu \mathrm{m}$.
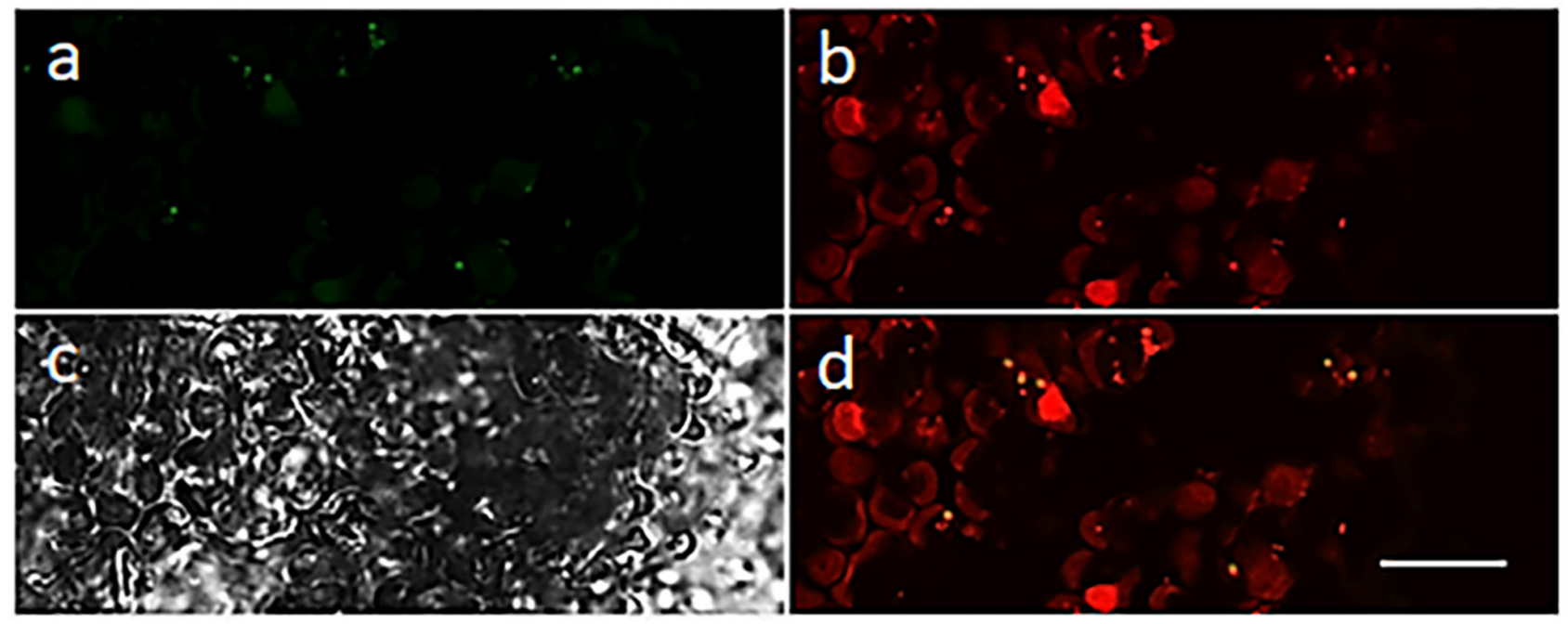

AtCAB6 $_{\mathrm{TP}}$-eGFP
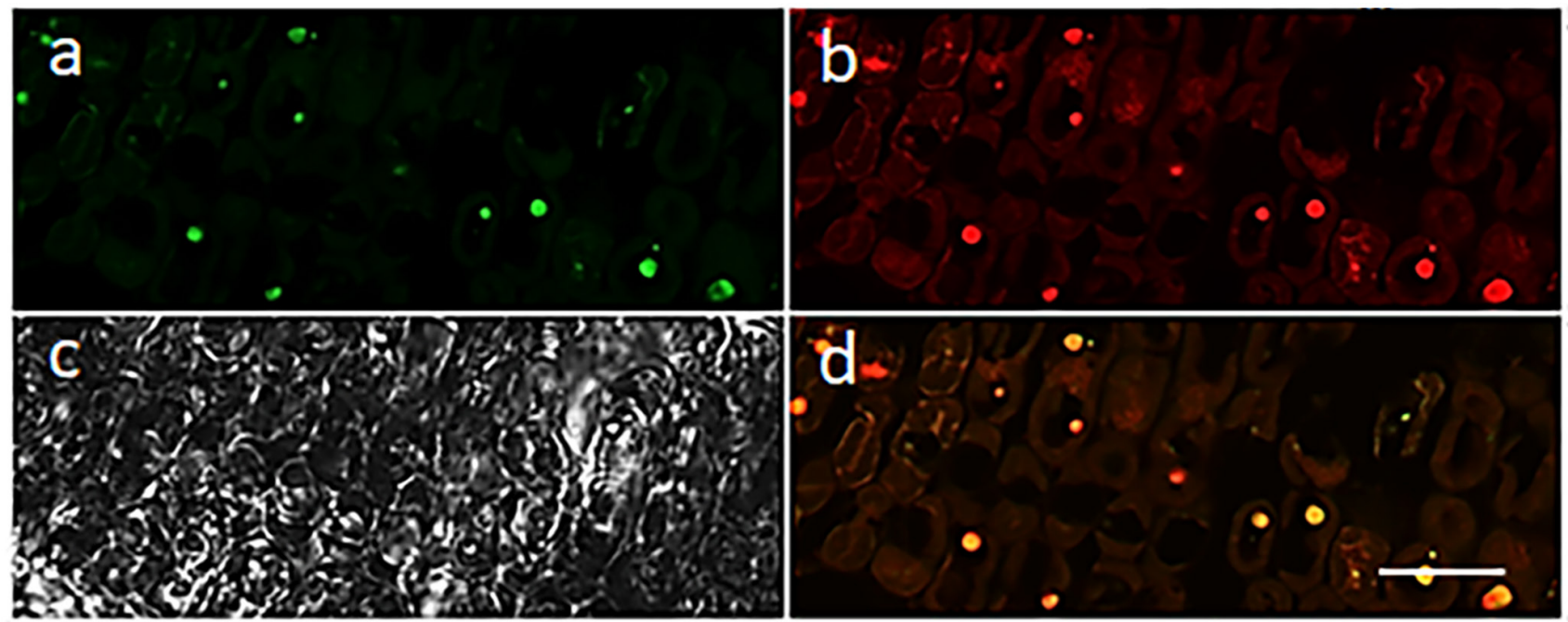


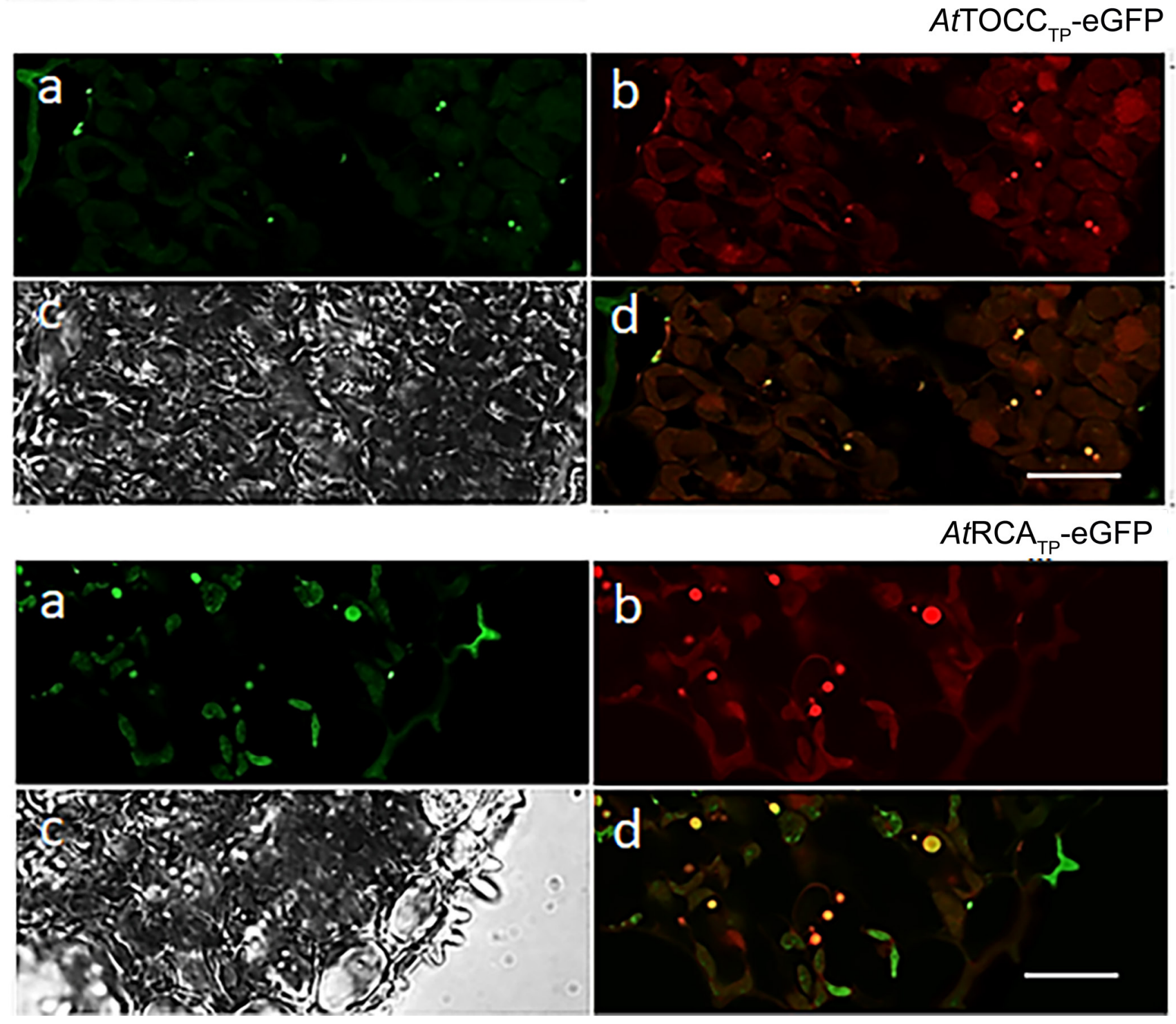


Supplemental Figure 9. Characteristics of $A$. thaliana and $O$. sativa Transit Peptides.

Relative abundance of Alanine, Serine and uncharged amino acids (Serine, Cysteine, Threonine, Tyrosine, Asparagine, Glutamine) in the minimal TPs and the mature protein.

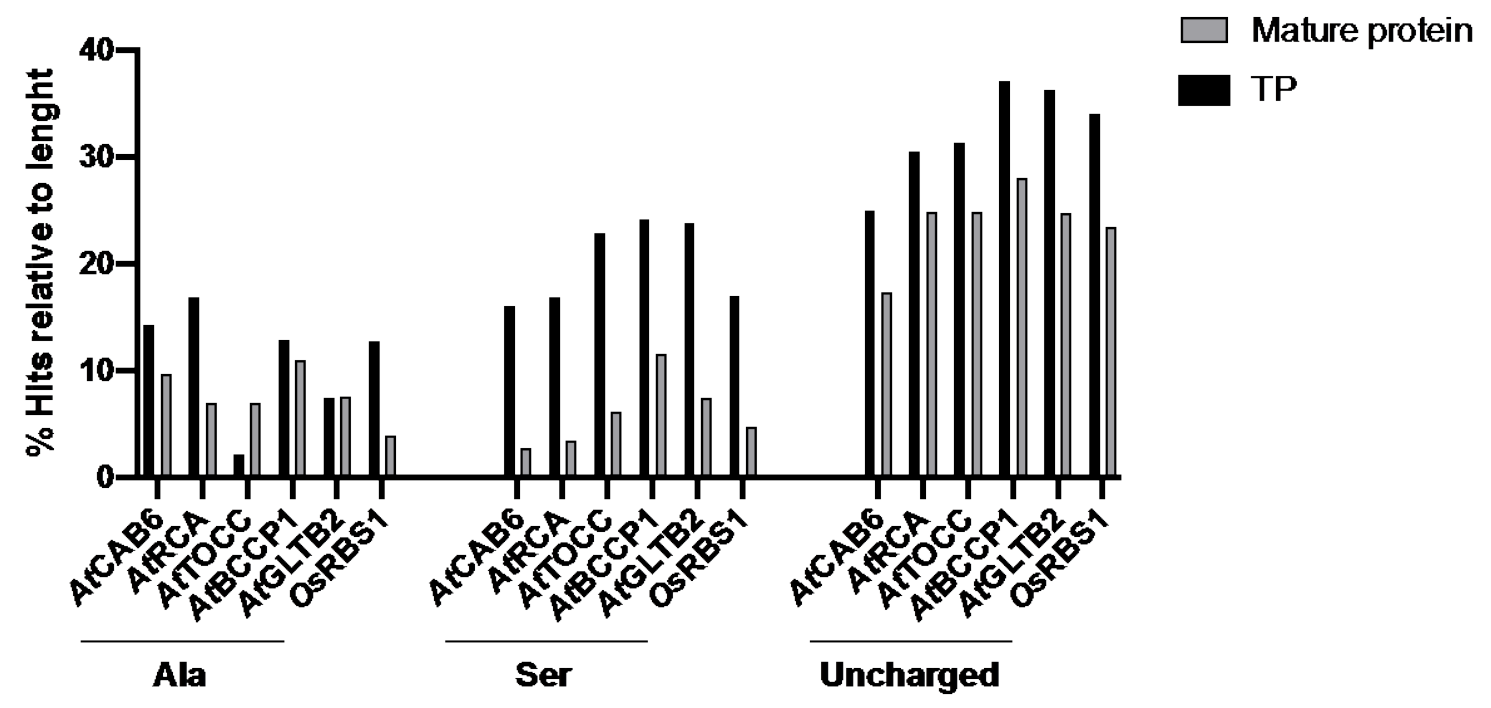


Additional File 1. Sequences of genes used in this thesis.

The complete nucleotide sequences of the nifH, nif $M$, nif $U$ and nifS yeast codon-optimized genes and tobacco synthetic designed genes have been deposited into GenBank under the accession numbers MN920692 to MN920699.

\begin{abstract}
$>$ AvnifH
ATGGCTATGCGTCAATGCGCCATCTACGGCAAAGGTGGTATCGGTAAGTCCACCACTACTCAGAACCTGG TGGCAGCCCTGGCTGAGATGGGCAAGAAGGTCATGATCGTTGGTTGTGACCCGAAAGCTGACTCCACCCG CCTGATCCTGCACTCCAAGGCCCAGAACACCATCATGGAAATGGCTGCCGAAGCCGGTACCGTGGAAGAT CTGGAGCTGGAAGATGTGCTGAAGGCTGGCTACGGCGGCGTCAAGTGCGTTGAGTCCGGTGGTCCGGAGC CGGGCGTTGGCTGCGCCGGCCGTGGTGTTATCACCGCCATCAACTTCCTGGAAGAGGAAGGCGCCTACGA AGATGATCTGGACTTCGTATTCTACGACGTGCTGGGCGACGTGGTGTGTGGCGGCTTCGCCATGCCGATC CGCGAGAACAAGGCCCAGGAAATCTACATCGTCTGCTCCGGTGAGATGATGGCCATGTACGCCGCCAACA ACATCTCCAAGGGCATCGTGAAGTATGCCAACTCCGGCAGCGTGCGTCTGGGCGGCCTGATCTGCAACAG CCGTAACACCGACCGCGAAGATGAGCTGATCATCGCTCTGGCCAACAAGCTGGGCACCCAGATGATCCAC TTCGTGCCGCGTGACAACGTCGTGCAGCGCGCCGAAATCCGCCGCATGACCGTGATCGAATACGATCCGA AAGCCAAGCAAGCCGACGAATACCGCGCTCTGGCCCGCAAGGTCGTAGACAACAAACTGCTGGTCATCCC GAACCCGATCACCATGGACGAGCTCGAAGAGCTGCTGATGGAATTCGGCATCATGGAAGTCGAAGATGAA TCCATCGTCGGCAAAACCGCCGAAGAAGTCTGA
\end{abstract}

$>$ Yeast codon optimization AvnifH

ATGGCCATGAGACAATGTGCTATCTACGGTAAAGGTGGTATTGGTAAAAGTACAACCACTCAAAACTTGG TTGCCGCTTTAGCAGAAATGGGTAAAAAGGTCATGATTGTTGGTTGCGATCCTAAGGCCGACTCAACTAG ATTGATATTACACTCCAAAGCTCAAAATACAATCATGGAAATGGCAGCCGAAGCAGGTACCGTTGAAGAT TTGGAATTGGAAGATGTATTGAAGGCCGGTTATGGTGGTGTAAAATGTGTCGAATCTGGTGGTCCTGAAC CAGGTGTCGGTTGCGCAGGTAGAGGTGTTATAACTGCCATCAATTTCTTGGAAGAAGAAGGTGCATACGA AGATGACTTAGATTTCGTTTTCTACGATGTATTGGGTGACGTCGTTTGTGGTGGTTTTGCTATGCCAATT AGAGAAAACAAGGCACAAGAAATCTATATAGTTTGCAGTGGTGAAATGATGGCTATGTACGCTGCAAACA ACATCTCTAAGGGTATAGTCAAGTACGCCAATTCTGGTTCAGTTAGATTGGGTGGTTTGATCTGTAACTC AAGAAACACTGATAGAGAAGATGAATTGATTATAGCCTTAGCTAACAAATTGGGTACACAAATGATCCAT TTCGTACCTAGAGATAATGTAGTCCAAAGAGCTGAAATCAGAAGAATGACAGTTATCGAATATGATCCAA AAGCTAAGCAAGCAGACGAATACAGAGCATTGGCCAGAAAGGTTGTAGATAATAAGTTGTTAGTTATACC TAACCCAATCACTATGGATGAATTGGAAGAATTGTTGATGGAATTCGGTATCATGGAAGTTGAAGATGAA TCTATAGTCGGTAAAACTGCTGAAGAAGTATAA

$>$ Tobacco synthetic design AvnifH

ATGGCAATGAGACAATGTGCTATATATGGTAAGGGCGGTATTGGAAAATCAACGACAACGCAGAATCTTG TTGCTGCTTTAGCCGAAATGGGGAAAAAGGTCATGATAGTTGGCTGTGATCCGAAGGCCGATTCAACTCG GTTAATACTTCATAGTAAAGCGCAAAATACAATCATGGAGATGGCAGCAGAAGCTGGAACTGTTGAGGAT CTAGAACTCGAGGACGTTCTTAAAGCAGGTTACGGTGGTGTTAAGTGCGTTGAATCTGGTGGACCTGAGC CAGGAGTGGGATGTGCAGGAAGGGGAGTTATTACAGCTATTAATTTCCTAGAAGAAGAGGGTGCTTACGA GGATGACTTGGACTTCGTATTCTATGATGTCTTAGGGGACGTAGTATGTGGCGGATTTGCCATGCCGATC AGAGAGAACAAGGCACAAGAGATCTATATTGTCTGCAGCGGTGAAATGATGGCTATGTACGCAGCTAACA ATATCTCCAAAGGGATAGTGAAGTATGCCAATTCAGGCTCAGTGAGGCTAGGTGGTTTAATCTGCAACTC TAGGAATACAGATCGAGAGGACGAACTAATCATCGCATTAGCAAATAAGTTGGGGACCCAGATGATACAT TTCGTCCCCAGAGATAATGTTGTGCAACGTGCTGAAATACGTAGAATGACTGTTATTGAGTATGATCCTA AGGCCAAACAGGCTGATGAGTACCGCGCTTTGGCGCGTAAAGTAGTTGATAACAAGCTTCTGGTAATTCC AAACCCTATTACTATGGATGAACTAGAGGAATTGTTAATGGAATTTGGGATTATGGAAGTTGAAGATGAA AGTATTGTGGGCAAAACCGCTGAGGAAGTGTAA 


\section{$>$ Rice codon optimization AvnifH}

ATGGCCATGAGGCAGTGCGCGATCTACGGCAAGGGCGGCATCGGCAAGAGCACCACTACCCAGAACCTCG TGGCGGCCCTGGCAGAGATGGGCAAGAAGGTCATGATCGTCGGCTGCGACCCGAAGGCCGATTCTACCCG GCTCATCCTGCACTCAAAGGCGCAGAATACCATCATGGAGATGGCGGCCGAGGCGGGAACAGTGGAGGAC CTCGAGCTGGAGGATGTCCTGAAGGCCGGATACGGCGGCGTGAAGTGCGTGGAGTCTGGCGGCCCTGAGC CAGGAGTGGGATGCGCAGGCCGCGGCGTCATCACAGCGATCAACTTCCTCGAGGAAGAGGGCGCCTACGA GGACGATCTGGACTTCGTGTTCTACGATGTGCTGGGCGACGTGGTCTGCGGCGGATTCGCCATGCCAATC CGGGAGAACAAGGCGCAGGAGATCTACATCGTGTGCTCGGGCGAGATGATGGCCATGTACGCCGCGAACA ATATCTCCAAGGGCATCGTGAAGTACGCAAATTCTGGAAGCGTCCGCCTCGGCGGCCTGATCTGCAACTC CCGGAATACGGACCGCGAGGATGAGCTCATTATCGCCCTGGCGAACAAGCTCGGCACCCAGATGATCCAT TTCGTGCCACGCGACAATGTGGTCCAGAGGGCGGAGATCCGCAGGATGACCGTCATCGAGTACGACCCTA AGGCCAAGCAGGCCGATGAGTACAGGGCACTGGCCCGGAAGGTGGTCGACAACAAGCTCCTCGTGATCCC TAATCCGATCACCATGGACGAGCTCGAGGAGCTCCTGATGGAGTTCGGCATCATGGAGGTGGAGGATGAG TCCATCGTCGGCAAGACGGCCGAGGAAGTGTGA

\section{$>$ Rice synthetic design AvnifH}

ATGGCCATGAGGCAGTGCGCGATCTACGGCAAGGGCGGCATCGGCAAGAGCACCACTACCCAGAACCTCG TGGCGGCCCTGGCAGAGATGGGCAAGAAGGTCATGATCGTCGGCTGCGACCCGAAGGCCGATTCTACCCG GCTCATCCTGCACTCAAAGGCGCAGAATACCATCATGGAGATGGCGGCCGAGGCGGGAACAGTGGAGGAC CTCGAGCTGGAGGATGTCCTGAAGGCCGGATACGGTGGCGTGAAGTGCGTGGAGTCTGGCGGCCCTGAGC CAGGAGTGGGATGCGCAGGCCGCGGAGTCATCACAGCGATCAACTTCCTCGAGGAAGAGGGCGCCTACGA GGACGATCTGGACTTCGTGTTCTACGATGTGCTGGGCGACGTGGTCTGTGGCGGATTCGCCATGCCAATC CGGGAGAACAAGGCGCAGGAGATCTACATCGTGTGCTCGGGCGAGATGATGGCCATGTACGCCGCGAACA ATATCTCCAAGGGCATCGTGAAGTACGCAAATTCTGGAAGCGTCCGCCTCGGCGGCCTGATCTGCAACTC CCGGAATACGGACCGCGAGGATGAGCTCATTATCGCCCTGGCGAACAAGCTCGGCACCCAGATGATCCAT TTCGTGCCACGCGACAATGTGGTCCAGAGGGCGGAGATCCGCAGGATGACCGTCATCGAGTACGACCCTA AGGCCAAGCAGGCCGATGAGTACAGGGCACTGGCCCGGAAGGTGGTCGACAACAAGCTCCTCGTGATCCC TAATCCGATCACCATGGACGAGCTCGAGGAGCTCCTGATGGAGTTCGGCATCATGGAGGTGGAGGATGAG TCCATCGTCGGCAAGACGGCCGAGGAAGTGTGA

\section{$>$ AvnifM}

ATGGCATCTGAGCGTCTCGCCGACGGCGACAGCCGCTATTACTTGCTGAAGGTCGCCCACGAGCAGTTCG GCTGCGCGCCCGGCGAGCTCAGCGAGGAGCAGCTCCAGCAGGCCGACCGCATCATCGGCCGGCAGAGGCA TATCGAGGACGCCGTGTTGCGCAGCCCCGATGCGATCGGTGTGGTGATCCCGCCCTCCCAGCTCGAAGAG GCCTGGGCACACATCGCCAGCCGCTACGAGAGTCCCGAGGCGCTACAGCAGGCCCTCGACGCGCAGGCGC TGGATGCTGCCGGCATGCGCGCCATGCTGGCCCGGGAGCTCAGGGTCGAGGCCGTTCTCGACTGCGTCTG CGCCGGCCTGCCGGAGATCAGCGACACCGATGTGAGCCTCTACTACTTCAACCACGCCGAGCAATTCAAG GTGCCCGCCCAGCACAAGGCCCGGCACATCCTGGTCACCATCAACGAGGATTTTCCGGAAAACACCCGCG AAGCCGCCCGGACGCGCATCGAGACAATCCTCAAGCGGCTGCGCGGCAAGCCGGAGCGCTTCGCCGAGCA GGCGATGAAGCACTCCGAATGTCCCACCGCGATGCAGGGCGGATTGCTCGGCGAGGTGGTCCCGGGAACG CTTTATCCCGAACTGGACGCCTGCCTGTTCCAGATGGCGCGGGGAGAACTGAGTCCGGTACTGGAATCGC CGATCGGTTTTCACGTGCTGTACTGCGAAAGCGTGAGCCCCGCCCGGCAGCTCACCCTCGAGGAGATCCT GCCGCGTCTGCGCGACAGGCTGCAGCTCCGGCAGCGCAAGGCGTATCAGCGCAAATGGCTGGAAAGCCTG CTCCAACAAAACGCTACTTTGGAGAACCTCGCCCATGGATGA

\section{$>$ Yeast codon optimization AvnifM}

ATGGCCTCAGAAAGATTAGCTGATGGTGACTCCAGATATTACTTGTTAAAAGTTGCCCATGAACAATTTG GTTGTGCTCCTGGTGAATTATCAGAAGAACAATTGCAACAAGCAGATAGAATTATAGGTAGACAAAGACA CATAGAAGATGCAGTTTTAAGATCACCAGACGCCATAGGTGTTGTCATCCCACCTTCCCAATTGGAAGAA GCTTGGGCACATATTGCTTCAAGATATGAATCCCCTGAAGCCTTGCAACAAGCTTTAGATGCCCAAGCTT TGGACGCTGCTGGTATGAGAGCAATGTTGGCCAGAGAATTAAGAGTTGAAGCTGTCTTAGATTGTGTCTG CGCAGGTTTGCCAGAAATTAGTGATACAGACGTATCTTTGTACTACTTCAACCATGCTGAACAATTCAAG GTACCAGCACAACATAAAGCCAGACACATATTGGTTACTATAAATGAAGATTTTCCTGAAAACACAAGAG AAGCCGCTAGAACCAGAATCGAAACTATCTTGAAGAGATTGAGAGGTAAACCAGAAAGATTCGCTGAACA AGCAATGAAACACTCTGAATGTCCTACAGCTATGCAAGGTGGTTTGTTAGGTGAAGTAGTTCCAGGTACC TTGTATCCTGAATTAGATGCATGCTTGTTTCAAATGGCCAGAGGTGAATTATCACCAGTTTTGGAATCCC CTATTGGTTTCCATGTTTTATACTGTGAATCCGTCAGTCCAGCAAGACAATTGACCTTGGAAGAAATCTT GCCTAGATTGAGAGATAGATTGCAATTGAGACAAAGAAAGGCTTACCAAAGAAAGTGGTTGGAATCTTTG TTGCAACAAAATGCTACCTTGGAAAACTTAGCACATGGTTAA 


\section{$>$ Tobacco synthetic design AvnifM}

ATGGCATCAGAAAGATTAGCTGACGGCGATTCGAGATATTATCTCTTGAAGGTTGCTCATGAACAGTTTG GATGTGCTCCGGGTGAGTTGAGCGAAGAGCAGTTACAACAAGCAGATAGGATTATAGGGAGACAAAGGCA TATCGAAGATGCCGTTCTGAGATCACCTGACGCAATAGGAGTAGTTATTCCTCCATCACAATTGGAAGAG GCTTGGGCACATATTGCATCTAGATATGAATCTCCCGAAGCTCTTCAGCAGGCGTTAGACGCTCAAGCAC TTGATGCTGCTGGAATGAGAGCTATGTTGGCCAGGGAGTTAAGAGTGGAAGCCGTGTTAGATTGCGTTTG TGCCGGGTTACCTGAAATATCTGATACTGATGTCAGTTTATACTACTTCAATCACGCTGAGCAATTCAAA GTACCTGCACAACATAAGGCTAGACATATCTTGGTGACGATTAACGAGGACTTTCCCGAGAACACTAGGG AAGCAGCTAGAACAAGAATTGAAACTATCTTAAAAAGGCTTAGAGGAAAACCGGAAAGGTTTGCAGAACA GGCTATGAAACACTCTGAGTGCCCTACAGCAATGCAGGGTGGTTTATTAGGTGAAGTGGTACCAGGAACT TTATATCCAGAATTAGATGCTTGCTTATTTCAGATGGCTAGGGGCGAATTATCCCCAGTATTAGAATCAC CCATAGGTTTCCACGTATTATATTGTGAGTCTGTTAGTCCGGCAAGACAGTTGACCCTAGAAGAGATCTT ACCTAGATTAAGGGATAGGCTGCAATTGAGACAGAGAAAGGCCTACCAAAGGAAATGGTTAGAAAGCTTG TTGCAGCAAAATGCAACATTAGAGAATTTGGCGCATGGATAA

\section{$>$ Rice codon optimization AvnifM}

ATGGCCTCAGAGAGGCTCGCGGACGGCGATTCGCGGTACTACCTCCTGAAGGTGGCACATGAGCAGTTCG GGTGCGCCCCTGGCGAGCTGTCTGAGGAGCAGCTGCAGCAGGCGGACCGCATCATCGGCCGCCAGAGGCA TATTGAGGACGCGGTCCTGAGGTCCCCGGATGCCATTGGAGTGGTCATCCCGCCAAGCCAGCTGGAGGAA GCCTGGGCGCATATCGCCTCCCGCTACGAGTCACCAGAGGCCCTCCAGCAGGCGCTGGACGCCCAGGCGC TCGATGCGGCCGGAATGAGGGCCATGCTGGCCAGGGAGCTGCGCGTGGAGGCGGTCCTGGACTGCGTGTG CGCCGGCCTCCCGGAGATCTCCGACACCGATGTCTCCCTGTACTACTTCAACCATGCCGAGCAGTTCAAG GTGCCAGCCCAGCACAAGGCGCGGCATATCCTCGTCACGATCAACGAGGATTTCCCTGAGAATACTAGGG AGGCGGCCAGGACCAGGATTGAGACAATCCTCAAGAGGCTGCGGGGCAAGCCTGAGAGGTTCGCAGAGCA GGCCATGAAGCATTCAGAGTGCCCTACAGCCATGCAGGGCGGCCTCCTGGGAGAGGTGGTGCCGGGCACG CTCTACCCTGAGCTGGATGCATGCCTGTTCCAGATGGCCCGCGGCGAGCTGAGCCCAGTGCTCGAGTCTC CTATCGGCTTCCACGTGCTGTACTGCGAGTCCGTCAGCCCTGCAAGGCAGCTCACCCTGGAGGAGATCCT GCCGCGCCTGAGGGATCGGCTCCAGCTGAGGCAGAGGAAGGCGTACCAGCGGAAGTGGCTCGAGAGCCTC CTGCAGCAGAACGCCACACTCGAGAATCTGGCGCACGGCTGA

\section{$>$ Rice synthetic design AvnifM}

ATGGCCTCAGAGAGGCTCGCGGACGGCGATTCGCGGTACTACCTCCTGAAGGTGGCACATGAGCAGTTCG GGTGCGCCCCTGGCGAGCTGTCTGAGGAGCAGCTGCAGCAGGCGGACCGCATCATCGGCCGCCAGAGGCA TATTGAGGACGCGGTCCTGAGGTCCCCGGATGCCATTGGAGTGGTCATCCCGCCAAGCCAGCTGGAGGAA GCCTGGGCGCATATCGCCTCCCGCTACGAGTCACCAGAGGCCCTCCAGCAGGCGCTGGACGCCCAGGCGC TCGATGCGGCCGGAATGAGGGCCATGCTGGCCAGGGAGCTGCGCGTGGAGGCGGTCCTGGACTGCGTGTG CGCCGGCCTCCCGGAGATCTCCGACACCGATGTCTCCCTGTACTACTTCAACCATGCCGAGCAGTTCAAG GTGCCAGCCCAGCACAAGGCGCGGCATATCCTCGTCACGATCAACGAGGATTTCCCTGAGAATACTAGGG AGGCGGCCAGGACCAGGATTGAGACAATCCTCAAGAGGCTGCGGGGCAAGCCTGAGAGGTTCGCAGAGCA GGCCATGAAGCACAGTGAGTGCCCTACAGCCATGCAGGGCGGCCTCCTGGGAGAGGTGGTGCCGGGCACG СTCTACCCTGAGCTGGATGCATGCCTGTTCCAGATGGCCCGCGGTGAGCTGAGCCCAGTGCTCGAGTCTC CTATCGGCTTCCACGTGCTGTACTGCGAGTCCGTCAGCCCTGCAAGGCAGCTCACCCTGGAGGAGATCCT GCCGCGCCTGAGGGATCGGCTCCAGCTGAGGCAGAGGAAGGCGTACCAGCGGAAGTGGCTCGAGAGCCTC CTGCAGCAGAACGCCACACTCGAGAATCTGGCGCACGGCTGA

\section{$>A v n i f U$}

ATGTGGGATTATTCGGAAAAAGTCAAAGAGCACTTCTACAACCCCAAGAATGCTGGAGCCGTGGAAGGCG CCAACGCCATCGGCGACGTCGGATCGCTGAGTTGCGGTGATGCGCTGCGCCTGACCCTGAAGGTGGACCC GGAAACCGACGTGATTCTGGATGCCGGCTTCCAGACCTTCGGCTGTGGTTCCGCCATCGCTTCCTCCTCG GCGCTGACCGAGATGGTCAAGGGCCTGACCCTGGACGAGGCGCTGAAGATCAGTAACCAGGACATCGCCG ACTACCTCGATGGCCTGCCGCCGGAGAAGATGCACTGCTCGGTGATGGGCCGCGAAGCCCTGCAGGCCGC GGTGGCCAACTACCGTGGCGAGACGATCGAGGACGACCACGAAGAGGGCGCGCTGATCTGCAAGTGCTTC GCCGTCGACGAGGTGATGGTCCGCGATACCATCCGTGCCAACAAGCTGTCTACCGTTGAGGACGTGACCA ATTACACCAAGGCCGGCGGTGGCTGCTCCGCCTGCCACGAGGCTATCGAGCGCGTGCTGACCGAAGAGCT GGCCGCTCGCGGTGAAGTCTTTGTCGCGGCCCCGATAAAGGCCAAGAAGAAGGTCAAGGTGCTCGCCCCC GAGCCGGCTCCCGCCCCGGTGGCCGAAGCCCCGGCGGCTGCCCCGAAGCTGAGCAACCTGCAGCGCATCC GTCGCATCGAGACAGTGCTGGCGGCGATCCGTCCGACCTTGCAGCGCGACAAGGGCGACGTCGAACTGAT CGATGTCGACGGCAAGAACGTTTATGTCAAGCTCACCGGCGCCTGCACCGGCTGCCAGATGGCCAGCATG ACCCTCGGCGGCATCCAGCAGCGCCTGATCGAGGAGCTCGGCGAGTTCGTCAAGGTGATTCCGGTCAGCG CTGCGGCTCACGCGCAGATGGAGGTCTGA 


\section{$>$ Yeast codon optimization AvnifU}

ATGTGGGACTACTCTGAAAAGGTTAAGGAACATTTCTACAATCCAAAGAACGCCGGTGCTGTAGAAGGTG CAAACGCCATTGGTGACGTTGGTTCATTATCCTGTGGTGACGCTTTGAGATTAACATTGAAAGTTGACCC TGAAACCGATGTCATCTTGGACGCAGGTTTTCAAACTTTCGGTTGCGGTTCTGCTATTGCATCTTCATCC GCTTTGACTGAAATGGTTAAGGGTTTGACATTGGATGAAGCATTGAAAATCTCAAACCAAGATATCGCTG ACTATTTGGATGGTTTGCCACCTGAAAAGATGCATTGTTCCGTCATGGGTAGAGAAGCCTTACAAGCTGC AGTAGCTAACTACAGAGGTGAAACCATTGAAGATGACCACGAAGAAGGTGCATTGATATGTAAATGCTTT GCCGTTGATGAAGTTATGGTCAGAGATACCATAAGAGCAAATAAGTTAAGTACTGTAGAAGATGTTACTA ACTACACAAAAGCTGGTGGTGGTTGTTCTGCTTGCCATGAAGCAATAGAAAGAGTTTTGACAGAAGAATT GGCCGCTAGAGGTGAAGTATTCGTTGCAGCCCCAATTAAAGCCAAAAAGAAAGTCAAGGTATTGGCTCCA GAACCTGCCCCAGCTCCTGTTGCAGAAGCCCCAGCTGCAGCCCCTAAGTTGTCAAATTTGCAAAGAATTA GAAGAATCGAAACAGTCTTGGCTGCAATAAGACCTACCTTGCAAAGAGACAAAGGTGACGTCGAATTAAT TGATGTAGACGGTAAAAATGTTTACGTCAAATTGACCGGTGCTTGTACTGGTTGCCAAATGGCATCCATG ACATTAGGTGGTATACAACAAAGATTGATCGAAGAATTGGGTGAGTTCGTCAAAGTTATCCCAGTCTCCG CTGCCGCACACGCCCAAATGGAAGTCTGA

\section{> Tobacco synthetic design AvnifU}

ATGTGGGATTACTCAGAGAAAGTTAAAGAGCACTTCTACAATCCCAAGAATGCAGGTGCTGTAGAAGGGG CAAATGCTATTGGGGACGTTGGATCTTTATCATGCGGTGATGCCTTAAGATTAACACTTAAAGTCGATCC TGAAACTGACGTTATTCTTGATGCTGGATTTCAGACCTTTGGTTGTGGATCAGCCATTGCTTCGTCCTCT GCGTTGACAGAGATGGTTAAGGGCTTGACATTGGACGAAGCATTAAAAATTAGTAATCAAGATATTGCCG ATTATTTGGATGGGTTGCCTCCCGAGAAGATGCATTGTTCAGTAATGGGAAGGGAAGCTTTACAAGCTGC GGTAGCAAATTATAGGGGAGAGACTATAGAGGACGATCACGAAGAGGGAGCTTTGATTTGCAAGTGCTTT GCTGTCGATGAGGTTATGGTGAGGGACACAATAAGAGCGAATAAGTTATCTACTGTTGAGGATGTTACAA ACTATACTAAAGCCGGCGGTGGCTGTTCTGCCTGCCATGAAGCGATTGAAAGAGTCTTAACTGAAGAATT GGCCGCAAGGGGTGAAGTGTTTGTCGCAGCTCCTATCAAAGCTAAGAAGAAGGTGAAAGTATTGGCTCCA GAACCTGCTCCTGCACCAGTTGCAGAAGCACCTGCGGCGGCACCAAAACTAAGTAACTTACAGAGGATTC GCAGAATAGAAACAGTGTTAGCTGCAATTAGACCAACATTGCAAAGAGACAAAGGAGATGTCGAATTGAT TGATGTTGATGGCAAAAACGTTTATGTTAAATTGACTGGTGCATGCACAGGTTGTCAAATGGCTTCAATG ACTTTAGGTGGAATTCAGCAAAGGTTGATCGAGGAATTAGGAGAATTCGTAAAGGTCATCCCAGTTAGTG CTGCTGCACATGCTCAGATGGAAGTATAA

\section{$>$ Rice codon optimization AvnifU}

ATGATGCCATCGGCGACGTCGGCTCACTGTCCTGCGGCGACGCCCTCCGCCTGACACTCAAGGTGGATCC GGAGACAGACGTCATCCTCGATGCCGGCTTCCAGACCTTCGGCTGCGGATCAGCGATCGCCTCCTCCTCC GCCCTGACTGAGATGGTGAAGGGCCTGACACTCGATGAGGCCCTCAAGATCTCTAACCAGGATATCGCCG ATTACCTGGACGGCCTCCCGCCAGAGAAGATGCACTGCTCAGTGATGGGAAGGGAGGCACTGCAGGCGGC CGTGGCCAATTACCGCGGCGAGACAATCGAGGACGATCATGAGGAAGGCGCCCTCATCTGCAAGTGCTTC GCGGTGGACGAGGTCATGGTGCGCGATACGATCCGCGCCAACAAGCTGTCCACCGTGGAGGACGTCACCA ATTACACAAAGGCTGGCGGCGGCTGCTCTGCATGCCATGAGGCCATTGAGAGGGTCCTGACAGAGGAGCT GGCAGCCAGGGGCGAGGTGTTCGTGGCAGCGCCAATCAAGGCCAAGAAGAAGGTGAAGGTCCTCGCGCCT GAGCCGGCCCCAGCCCCGGTGGCGGAGGCCCCTGCGGCCGCACCAAAGCTGAGCAACCTCCAGAGGATCC GCAGGATCGAGACAGTGCTCGCCGCAATCAGGCCTACCCTCCAGCGGGATAAGGGCGACGTGGAGCTGAT CGACGTCGATGGCAAGAATGTCTACGTGAAGCTGACCGGAGCATGCACTGGATGCCAGATGGCGTCCATG ACTCTCGGCGGCATCCAGCAGCGGCTGATCGAGGAGCTCGGCGAGTTCGTGAAGGTCATCCCGGTGTCCG CCGCAGCCCATGCCCAGATGGAGGTCTGA 


\section{$>$ Rice synthetic design AvnifU}

ATGTGGGACTACAGCGAGAAGGTGAAGGAGCACTTCTACAATCCGAAGAATGCGGGAGCCGTGGAGGGAG CAAATGCCATCGGCGACGTCGGCTCACTGTCCTGTGGGGACGCCCTCCGCCTGACACTCAAGGTGGATCC GGAGACAGACGTCATCCTCGATGCCGGCTTCCAGACCTTCGGCTGCGGATCAGCGATCGCCTCCTCCTCC GCCCTGACTGAGATGGTGAAGGGCCTGACACTCGATGAGGCCCTCAAGATCTCTAACCAGGATATCGCCG ATTACCTGGACGGCCTCCCGCCAGAGAAGATGCACTGCTCAGTGATGGGAAGGGAGGCACTGCAGGCGGC CGTGGCCAATTACCGCGGAGAGACAATCGAGGACGATCATGAGGAAGGCGCCCTCATCTGCAAGTGCTTC GCGGTGGACGAGGTCATGGTGCGCGATACGATCCGCGCCAACAAGCTGTCCACCGTGGAGGACGTCACCA ATTACACAAAGGCTGGCGGTGGCTGCTCTGCATGCCATGAGGCCATTGAGAGGGTCCTGACAGAGGAGCT GGCAGCCAGGGGCGAGGTGTTCGTGGCAGCGCCAATCAAGGCCAAGAAGAAGGTGAAGGTCCTCGCGCCT GAGCCGGCCCCAGCCCCGGTGGCGGAGGCCCCTGCGGCCGCACCAAAGCTGAGCAACCTCCAGAGGATCC GCAGTATTGAGACAGTGCTCGCCGCAATCAGGCCTACCCTCCAGCGGGATAAGGGCGACGTGGAGCTAAT CGACGTCGATGGCAAGAATGTCTACGTGAAGCTGACCGGAGCATGCACTGGATGCCAGATGGCGTCCATG ACTCTCGGCGGCATCCAGCAGCGGCTCATTGAGGAGCTCGGCGAGTTCGTGAAGGTCATCCCGGTGTCCG CCGCAGCCCATGCCCAGATGGAGGTCTGA

\section{$>$ AvnifS}

ATGGCTGACGTCTATCTCGATAACAACGCCACCACCCGGGTGGACGACGAAATCGTCCAGGCCATGCTGC CGTTCTTCACCGAACAGTTCGGCAACCCCTCGTCGCTGCACAGCTTCGGCAACCAGGTCGGCATGGCGCT GAAGAAGGCCCGCCAGAGCGTGCAGAAACTGCTCGGTGCCGAACACGATTCGGAAATCGTCTTTACCTCC TGCGGCACCGAGTCGGACTCCACCGCGATCCTCTCGGCGCTCAAGGCCCAGCCCGAACGCAAGACCGTCA TCACCACCGTGGTCGAACACCCGGCCGTCCTCAGCCTGTGCGATTACCTCGCCAGTGAGGGCTACACCGT GCACAAGCTGCCGGTGGACAAGAAAGGCCGTCTGGATCTGGAGCATTACGCCAGCCTGCTGACCGACGAT GTCGCCGTGGTGTCGGTGATGTGGGCCAACAACGAGACAGGTACCCTGTTCCCGATCGAAGAGATGGCGC GCCTGGCCGACGACGCCGGCATCATGTTTCACACCGATGCCGTGCAGGCCGTGGGCAAGGTACCGATCGA CCTGAAGAATTCGTCGATCCACATGCTTTCGCTGTCGGGCCACAAGCTGCACGCGCCCAAAGGTGTCGGC GTGCTCTATCTGCGCCGCGGCACCCGCTTCCGTCCGCTGCTGCGCGGTGGTCACCAGGAGCGCGGCCGTC GTGCCGGCACCGAGAACGCCGCCTCCATCATCGGCCTGGGTGTCGCCGCCGAACGCGCGCTGCAGTTCAT GGAGCACGAGAACACCGAGGTCAAGCGCCTGCGCGACAAGCTGGAGGCCGGTATCCTCGCCGTCGTGCCC CATGCCTTCGTCACCGGCGATCCGGACAACCGCTTGCCCAACACCGCCAACATCGCGTTCGAGTACATCG AGGGCGAGGCCATCCTGCTGCTGCTGAACAAGGTCGGCATCGCCGCCTCCAGCGGTTCGGCCTGCACCTC CGGCTCCTTGGAGCCCTCCCACGTGATGCGCGCCATGGACATTCCCTATACTGCCGCCCACGGCACCGTG CGCTTCTCCCTGTCGCGCTACACCACCGAGGAGGAGATCGACCGGGTGATCCGCGAGGTTCCGCCGATCG TGGCCCAGTTGCGCAAGCTGTCGCCCTACTGGAGCGGCAACGGTCCGGTGGAGGACCCGGGCAAGGCCTT CGCTCCGGTCTACGGCTGA

\section{$>$ Yeast codon optimization AvnifS}

ATGGCCGACGTTTACTTGGATAATAACGCTACTACAAGAGTCGATGACGAAATAGTACAAGCTATGTTGC CATTTTTCACAGAACAATTCGGTAACCCTTCCAGTTTGCATTCCTTCGGTAACCAAGTTGGTATGGCCTT GAAGAAAGCTAGACAATCTGTCCAAAAATTGTTAGGTGCAGAACACGATTCCGAAATCGTTTTTACCAGT TGTGGTACTGAATCTGACTCAACCGCCATTTTGTCTGCCTTAAAAGCTCAACCAGAAAGAAAGACTGTCA TAACCACTGTTGTCGAACATCCTGCAGTATTGTCTTTATGCGATTATTTGGCCTCAGAAGGTTACACTGT TCATAAGTTACCAGTCGATAAAAAGGGTAGATTGGACTTAGAACACTATGCTTCCTTGTTAACAGATGAC GTAGCTGTAGTTAGTGTTATGTGGGCAAATAACGAAACTGGTACATTGTTTCCAATTGAAGAAATGGCAA GATTAGCCGATGACGCTGGTATAATGTTCCATACTGATGCAGTACAAGCCGTTGGTAAAGTCCCTATAGA CTTGAAGAACTCGTCAATCCACATGTTGTCCTTAAGTGGTCATAAATTGCACGCTCCAAAGGGTGTTGGT GTCTTGTACTTAAGAAGAGGTACAAGATTCAGACCTTTGTTAAGAGGTGGTCATCAAGAAAGAGGTAGAA GAGCCGGTACTGAAAATGCTGCATCTATTATAGGTTTGGGTGTTGCCGCTGAAAGAGCTTTACAATTCAT GGAACATGAAAACACTGAAGTTAAGAGATTGCGTGATAAGTTAGAAGCAGGTATTTTGGCCGTCGTACCA CACGCATTTGTTACTGGTGACCCAGACAATAGATTACCTAACACAGCTAACATAGCATTCGAATACATCG AAGGTGAAGCTATCTTGTTGTTGTTGAACAAAGTTGGTATAGCAGCCTCCAGTGGTTCTGCTTGTACATC TGGTTCATTGGAACCATCACATGTTATGAGAGCAATGGATATTCCTTATACAGCTGCACACGGTACTGTT AGATTTTCTTTGAGTAGATACACAACCGAAGAAGAAATTGATAGAGTCATTAGAGAAGTACCACCTATTG TTGCTCAATTGAGAAAATTGTCTCCTTACTGGTCAGGTAATGGTCCTGTTGAAGATCCTGGTAAAGCCTT TGCTCCTGTCTATGGTTGA 


\title{
$>$ Tobacco synthetic design AvnifS
}

ATGGCAGACGTTTATTTGGACAACAATGCCACTACAAGAGTTGACGATGAGATTGTTCAGGCAATGTTAC CATTCTTTACTGAACAATTTGGTAATCCAAGTAGTTTGCATAGTTTCGGAAATCAAGTGGGTATGGCGCT TAAAAAGGCCCGTCAGAGTGTACAGAAACTACTGGGCGCGGAACACGATAGTGAGATCGTGTTCACGTCA TGCGGCACAGAGTCTGACAGCACCGCAATCTTGTCCGCATTAAAAGCACAGCCTGAGCGTAAGACTGTCA TAACCACTGTAGTTGAGCATCCTGCTGTTCTATCATTATGTGATTATCTTGCATCTGAAGGATACACTGT GCATAAATTACCAGTGGATAAGAAGGGACGATTGGACCTCGAACACTATGCTTCCTTATTAACTGATGAC GTTGCAGTTGTCAGTGTCATGTGGGCCAATAACGAAACAGGTACATTATTTCCAATAGAGGAAATGGCTA GATTGGCGGATGATGCAGGGATAATGTTTCATACAGATGCCGTCCAAGCCGTCGGGAAAGTACCGATCGA TTTGAAGAATAGTTCAATACATATGTTATCGTTAAGCGGACATAAGCTGCACGCACCTAAAGGTGTTGGA GTTCTCTACTTACGTAGGGGTACGCGATTTCGACCCCTGCTCAGAGGTGGTCATCAAGAGAGAGGGCGAA GAGCCGGGACGGAAAATGCAGCATCTATTATTGGCTTAGGCGTGGCTGCTGAAAGAGCTTTACAATTCAT GGAACATGAGAATACCGAGGTAAAACGGCTTCGTGATAAGTTAGAGGCTGGCATTCTCGCCGTAGTCCCA CATGCCTTCGTCACAGGAGATCCTGATAACCGATTACCTAACACAGCAAATATAGCGTTTGAATATATTG AAGGTGAAGCTATACTATTGTTACTAAATAAGGTAGGAATTGCGGCGAGTTCCGGTTCAGCATGCACTTC AGGTTCACTAGAGCCTTCACACGTTATGCGAGCTATGGATATTCCGTATACAGCAGCTCATGGTACTGTT CGCTTTAGTCTTAGTCGGTACACAACCGAAGAAGAGATAGACAGGGTAATTAGGGAAGTTCCACCTATAG TCGCACAATTACGCAAGCTGTCACCATATTGGTCTGGGAATGGTCCTGTGGAAGATCCAGGAAAAGCTTT CGCGCCCGTTTACGGATAA

\begin{abstract}
$>$ Rice codon optimization AvnifS
ATGGCGGACGTGTACCTCGATAACAATGCCACCACACGGGTGGACGATGAGATCGTCCAGGCCATGCTCC CGTTCTTCACAGAGCAGTTCGGCAACCCATCCAGCCTCCACTCCTTCGGCAATCAAGTGGGCATGGCGCT GAAGAAGGCAAGGCAGTCTGTCCAGAAGCTCCTGGGCGCGGAGCATGACAGCGAGATCGTGTTCACGTCT TGCGGCACCGAGAGCGATTCTACGGCCATCCTCTCTGCCCTGAAGGCGCAGCCAGAGCGCAAGACCGTGA TCACGACCGTGGTCGAGCACCCTGCGGTCCTCTCACTGTGCGACTACCTCGCCTCGGAGGGCTACACCGT GCACAAGCTGCCGGTCGACAAGAAGGGCCGCCTCGATCTGGAGCATTACGCGTCCCTCCTGACAGACGAT GTGGCGGTGGTCAGCGTCATGTGGGCCAACAACGAGACAGGCACACTCTTCCCAATCGAGGAGATGGCAA GGCTGGCCGATGATGCAGGCATCATGTTCCACACTGATGCCGTGCAGGCGGTGGGCAAGGTGCCTATCGA TCTCAAGAACTCTTCAATCCATATGCTCTCACTGTCCGGACACAAGCTCCATGCGCCTAAGGGAGTGGGA GTCCTCTACCTGCGCAGGGGCACTAGGTTCCGGCCACTCCTGAGGGGCGGCCATCAGGAGCGGGGCCGCA GGGCCGGAACCGAGAATGCAGCGTCCATCATCGGCCTCGGAGTGGCGGCCGAGAGGGCACTGCAGTTCAT GGAGCACGAGAATACGGAGGTCAAGCGCCTCAGGGACAAGCTCGAGGCCGGAATCCTGGCAGTGGTCCCA CATGCGTTCGTGACCGGCGACCCAGATAACCGGCTGCCTAACACAGCGAATATCGCCTTCGAGTACATCG AGGGCGAGGCCATCCTCCTGCTCCTGAATAAGGTGGGCATTGCCGCGTCGTCCGGATCTGCCTGCACCTC CGGCAGCCTCGAGCCTTCACACGTGATGAGGGCCATGGACATCCCGTACACAGCCGCGCATGGCACGGTC AGGTTCTCTCTCTCACGGTACACAACGGAGGAAGAGATCGATCGGGTCATCAGGGAGGTGCCGCCAATTG TGGCACAGCTCCGCAAGCTGTCGCCTTACTGGTCCGGCAATGGCCCTGTGGAGGATCCTGGCAAGGCATT CGCCCCTGTCTACGGATGA
\end{abstract}

\section{$>$ Rice synthetic design AvnifS}

ATGGCGGACGTGTACCTCGATAACAATGCCACCACACGGGTGGACGATGAGATCGTCCAGGCCATGCTCC CGTTCTTCACAGAGCAGTTCGGCAACCCATCCAGCCTCCACTCCTTCGGCAATCAAGTGGGCATGGCGCT GAAGAAGGCAAGGCAGTCTGTCCAGAAGCTCCTGGGCGCGGAGCATGACAGCGAGATCGTGTTCACGTCT TGCGGCACCGAGAGCGATTCTACGGCCATCCTCTCTGCCCTGAAGGCGCAGCCAGAGCGCAAGACCGTGA TCACGACCGTGGTCGAGCACCCTGCGGTCCTCTCACTGTGCGACTACCTCGCCTCGGAGGGCTACACCGT GCACAAGCTGCCGGTCGACAAGAAGGGCCGCCTCGATCTGGAGCATTACGCGTCCCTCCTGACAGACGAT GTGGCGGTGGTCAGCGTCATGTGGGCCAACAACGAGACAGGCACACTCTTCCCAATCGAGGAGATGGCAA GGCTGGCCGATGATGCAGGCATCATGTTCCACACTGATGCCGTGCAGGCGGTGGGCAAGGTGCCTATCGA TCTCAAGAACTCTTCAATCCATATGCTCTCACTGTCCGGACACAAGCTCCATGCGCCTAAGGGAGTGGGA GTCCTCTACCTGCGCAGGGGCACTAGGTTCCGGCCACTCCTGAGGGGCGGCCATCAGGAGCGGGGCCGCA GGGCCGGAACCGAGAATGCAGCGTCCATCATCGGCCTCGGAGTGGCGGCCGAGAGGGCACTGCAGTTCAT GGAGCACGAGAATACGGAGGTCAAGCGCCTCAGGGACAAGCTCGAGGCCGGAATCCTGGCAGTGGTCCCA CATGCGTTCGTGACCGGCGACCCAGATAACCGGCTGCCTAACACAGCGAATATCGCCTTCGAGTACATCG AGGGCGAGGCCATCCTCCTGCTCCTGAATAAGGTGGGCATTGCCGCGTCGTCCGGATCTGCCTGCACCTC CGGCAGCCTCGAGCCTTCACACGTGATGAGGGCCATGGACATCCCGTACACAGCCGCGCATGGCACGGTC AGGTTCTCTCTCTCACGGTACACAACGGAGGAAGAAATAGATCGGGTCATCAGGGAGGTGCCGCCAATTG TGGCACAGCTCCGCAAGCTGTCGCCTTACTGGTCCGGCAATGGCCCTGTGGAGGATCCTGGCAAGGCATT CGCCCCTGTCTACGGATGA 


\section{References for Supplemental Material}

1. Engler, C. et al. A Golden Gate Modular Cloning Toolbox for Plants. ACS Synth. Biol. 3, 839-843 (2014).

2. Sarrion-Perdigones, A. et al. GoldenBraid 2.0: A Comprehensive DNA Assembly Framework for Plant Synthetic Biology. PLANT Physiol. 162, 1618-1631 (2013).

3. Sudhakar, D. et al. Expression and immunolocalisation of the snowdrop lectin, GNA in transgenic rice plants. Transgenic Res. 7, 371-378 (1998).

4. Ji, G. et al. Predictive modeling of plant messenger RNA polyadenylation sites. $B M C$ Bioinformatics 8, 43 (2007).

5. Shen, Y. et al. Genome level analysis of rice mRNA 3???-end processing signals and alternative polyadenylation. Nucleic Acids Res. 36, 3150-3161 (2008).

6. Jackson, M. A., Sternes, P. R., Mudge, S. R., Graham, M. W. \& Birch, R. G. Design rules for efficient transgene expression in plants. Plant Biotechnol. J. 12, 925-933 (2014).

7. Klee, H. J., Muskopf, Y. M. \& Gasser, C. S. Cloning of an Arabidopsis thaliana gene encoding 5-enolpyruvylshikimate-3-phosphate synthase: sequence analysis and manipulation to obtain glyphosate-tolerant plants. MGG Mol. Gen. Genet. 210, 437-442 (1987).

8. Lee, D. W. et al. Arabidopsis Nuclear-Encoded Plastid Transit Peptides Contain Multiple Sequence Subgroups with Distinctive Chloroplast-Targeting Sequence Motifs. Plant Cell 20, 1603-1622 (2008).

9. Lee, D. W. \& Hwang, I. Transient expression and analysis of chloroplast proteins in arabidopsis protoplasts. Methods Mol. Biol. 774, 59-71 (2011).

10. Mazur, B. J. \& Chui, C. F. Sequence of a genomic DNA clone for the small subunit of ribulose bis-phosphate carboxylase-oxygenase from tobacco. Nucleic Acids Res. 13, 2373-2386 (1985).

11. Coruzzi, G., Broglie, R., Edwards, C. \& Chua, N. H. Tissue-specific and light-regulated expression of a pea nuclear gene encoding the small subunit of ribulose-1,5-bisphosphate carboxylase. EMBO J. 3, 1671-1679 (1984).

12. Kim, S. et al. Arabidopsis thaliana Rubisco small subunit transit peptide increases the accumulation of Thermotoga maritima endoglucanase Cel5A in chloroplasts of transgenic tobacco plants. Transgenic Res. 19, 489-497 (2010).

13. Yonekura-Sakakibara, K., Ashikari, T., Tanaka, Y., Kusumi, T. A. \& Hase, T. Molecular characterization of tobacco sulfite reductase: Enzyme purification, gene cloning, and gene expression analysis. J. Biochem. 124, 615-621 (1998).

14. Nakata, M. T. et al. Plastid translation is essential for lateral root stem cell patterning in Arabidopsis thaliana. Biol. Open 7, (2018).

15. Shen, B.-R. et al. Engineering a New Chloroplastic Photorespiratory Bypass to Increase Photosynthetic Efficiency and Productivity in Rice. Mol. Plant 12, 199-214 (2019).

16. Schneider, C. A., Rasband, W. S. \& Eliceiri, K. W. NIH Image to ImageJ: 25 years of image analysis. Nat. Methods 9, 671-5 (2012).

17. Waese, J. et al. ePlant: Visualizing and exploring multiple levels of data for hypothesis generation in plant biology. Plant Cell 29, 1806-1821 (2017).

18. Thimm, O. et al. mapman: a user-driven tool to display genomics data sets onto diagrams of metabolic pathways and other biological processes. Plant J. 37, 914-939 (2004). 

